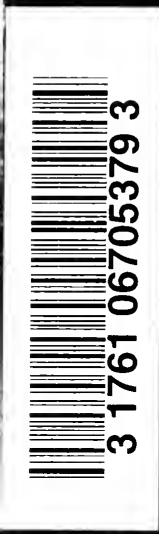

1.1. 



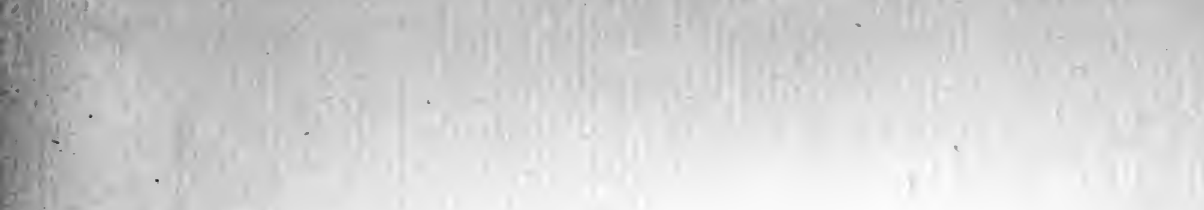

$\frac{1}{1+1}$

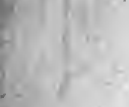

\section{th Ih}

$1+1-1+2+2$

-11 -1

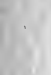

I)

,

(5)

H:

$2+\frac{1}{2}$

13

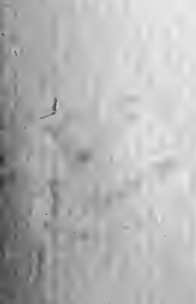

(2)

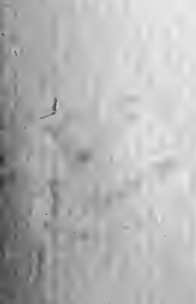

t.

. .2

$\sqrt{2}+2=0$

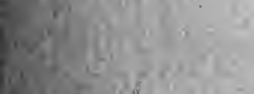

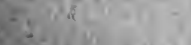

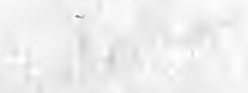

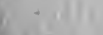


Digitized by the Internet Archive in 2007 with funding from Microsoft Corporation 


\section{OXFORD MEDICAL PUBLICATIONS}

\section{SPRAINS AND ALLIED IN.JURIES OF JOINTS}

SECOND EDITION 
OXFORD: HORACE HART

PRINTER TO THE UNIVERSITY 


\section{SPRAINS AND ALLIED INJURIES OF JOINTS}

BY

R. H. ANGLIN WHI'TELOCKE

M.D., M.C. (EDIn.), F.R.C.S. (ENG.)

HONORARY SURGEON TO THE RADCIIFFE INFIRMARY AND COUNTY

HOSPITAL AT OXFORD; I.ITCHFIELD LECTURER IN SURGERY IN THE UNIVERSITY

SECOND EDITION

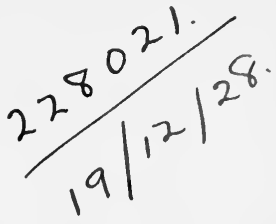

\section{LONDON}

HENRY FROWDE Oxford University Press
HODDER \& STOUGHTON

Warwick Square, E.C 



\section{PREFACE TO SECOND EDITION}

Encouraged by the favourable reviews of the Medical Press both in this country and America, an effort has been made to make this new edition even more useful.

The general character of the work has been in no way changed.

The alterations made are merely with a view to amplification.

Throughout the book slight additions have been made and reports of new cases interpolated for the purposes of clearness and better illustration. Nearly forty pages have been added to the letterpress, whilst the illustrations have been increased from sixty-nine to eighty-five.

In Chapter V an account of Schlatter's disease, of sprains in relation to exostoses, and of partial displacement of the patella outwards, have been added.

In place of the appendix Chapter $\mathrm{X}$ has been introduced.

It was thought that a chapter dealing with such special forms of treatment as radiant heat, injections 


\section{vi PREFACE TO SECOND EDITION}

of fibrolysin, Bier's hyperæmic methods, mechanotherapy, and kataphoresis would be a useful addition to the ordinary treatment of sprains.

A few of the old illustrations have been replaced by new and better ones.

With two exceptions, all the new radiograms and photographs, as in the last edition, were taken by my colleague, Mr. R. H. Sankey. Figs. 14 and 25 are from radiograms kindly lent by Mr. J. Keogh Murphy.

I wish to take this opportunity of thanking my friends Dr. Jamieson Hurry and Miss C. O. Stevens, for their kind criticisms and for reading the letterpress, as well as Messrs. Sankey and Keogh Murphy for their assistance with the illustrations.

\section{R. HENRY ANGLIN WHITELOCKE.}

St. Giles' Gate, Oxford, June 10, 1910. 


\section{PREFACE TO THE ORIGINAL EDITION}

Three events in recent times have suggested the writing of this volume, and seem to justify its production.

I. The more extensive employment of radiography by surgeons as an aid to diagnosis has led to the discovery that many injuries which formerly were believed to be, and treated as simple sprains, are in reality injuries to bones, and therefore of more serious consequence.

II. The passing of the new Workmen's Compensation Act and its operations have not only drawn the attention of the lay public to these accidents, but have placed them on a more important footing in the estimation of the profession than formerly.

It is incumbent upon every practitioner that he should not only be able to treat these affections successfully, but that he should also be able to give a reliable prognosis concerning them.

Every succeeding day brings the medico-legal aspect of the subject into greater prominence, for not only is the question of compensation an important 


\section{viii PREFACE TO THE ORIGINAL EDITION}

one with Insurance Companies, but it is found that it is in this particular class of slight or minor injuries that the greatest difficulty is experienced in estimating the exact monetary value of a claim.

III. At the present time not only do the uneducated and those of the lower orders of society seek the advice of unqualified practitioners only too frequently, but it has become the fashion for the "smart set' to flock in increasing numbers to the waitingrooms of those who proclaim loudest their skill, and who perhaps pay most for their daily advertisements.

No claim is made for the present treatise that it represents all that is known on the subject. The purpose is rather to supply certain details, operative and otherwise, which are not to be found in the ordinary textbooks on Surgery.

It is the embodiment for the most part of the writer's own personal experience, and as such may be helpful to some who may not have had his almost unique opportunities for dealing with athletic and other injuries.

As in the text certain terms, as 'wobbly-joint', ' weak-joint ', 'drop-top', ' mallet-finger', have been introduced, some explanation seems necessary. Everyday usage makes it almost impossible to do without them, for to the lay mind they convey a definite and specific meaning, and are expressions 
PREFACE TO THE ORIGINAL EDITION ix presumably introduced by and remaining in the daily use of bone-setters and others.

Regret must also be expressed that the idioms 'rider's-sprain', 'tennis-leg', and many others similar, should have crept into surgical literature.

Certain repetitions, especially in relation to anatomical points, are to be found; they are made with a view to the accentuation of important facts.

The radiograms, with the single exception of Fig. 18, kindly lent by Mr. Edwin Dening, together with most of the photographs, were taken for me by my friend and colleague, Mr. R. H. Sankey, M.A., M.B., B.Ch., Oxon., Honorary Radiographer to the Radcliffe Infirmary and County Hospital at Oxford.

I wish to take this opportunity of expressing to each of those who have so kindly and ably contributed to the illustrations, my best thanks for the trouble and skill they have bestowed, and to acknowledge my deep indebtedness to those of my undergraduate friends who have kindly allowed themselves to be photographed.

I wish also to place on record my indebtedness to those members of the profession who from time to time have written on these and kindred subjects, and especially to Sir William Bennett, Professor Howard Marsh, and Mr. L. B. Rawling, to each of whom $I$ have made reference in the text. To my 


\section{$x$ PREFACE TO THE ORIGINAL EDITION}

friends at 35 Harley Street, Drs. G. C. Cathcart and Theodore Thompson, for reading the proofs, and to the authors of that excellent, Textbook on Surgery, Messrs. Thomson and Miles, whose arrangement of surgical anatomy I have in a measure adopted,"my best thanks are here offered.

St. Giles' Gate, Oxford, February 1909. 


\title{
CONTEN'TS
}

\author{
CHAPTER I
}

INTRODUCTORY

The Structure and Function of Joints.

\section{CHAPTER II}

The general consideration of Sprains

Introduction-Definition-Classification-Articular Sprains

-Muscular Sprains-Simple Sprain-Strain-Complicated Sprain-Sprain-fracture-Sprain with fracture-Spraindislocation-Sprain with gross nerve lesion-Hæmatomata Diagnosis-Prognosis-Treatment-of Strains-of Sprains with immediate swelling-Elastic Pressure-Method of Application-Special advantages-Active Voluntary Movements-Passive Movements-Constitutional Complications-Tension in joints after sprains-Arthrotomy and digital exploration-Sprain complicated with injury to the nerves-Sprains with fractures.

\section{CHAPTER III}

\section{The Sequela of Sprains}

Preventable sequelce-Stiff and painful joints-Atrophy and wasting of muscles acting on joints-Recurring or relapsing synovitis-A relaxed condition of joints known as ' wobblyjoint' or 'weak-joint'-Persistent pain in a jointCrippled or deformed joint-Improperly united muscles and tendons-Ankylosis.

Unavoidable sequelo-Local paresis or paralysis-Ossifying myositis-Osteo-arthritis-Loose Bodies in Joints-Treatment for removal of Loose Bodies. . 


\section{CHAPTER IV}

Individual Articular Sprains.$\quad \cdot \quad \cdot \quad \cdot \quad \cdot$

Of the Upper Extremity-Sterno-clavicular joint-Acromioclavicular-Shoulder-Elbow-'Dislocation by elongation '-Wrist-Teno-synovitis in fore-arm-Fracture of Scaphoid bone-Fracture of the metacarpal bones simulating sprain-Sprain of the thumb-'Drop-top' or 'mallet-finger'-Sprains of' the finger-joints-Fracture of first phalanx of little finger.

Of the Louer Extremity-Hip-joint-Ankle-joint-' Metatarsal pain'-Tarso-metatarsal joint-Fracture of the tuberosity of the fifth metatarsal bone.

Sprains of the Trunk-of the side (intercostal muscles)sacro-coccygeal sprain-coccygodynia.

\section{CHAPTER V}

Sprains in the Region of the Knee-Joint

Surgical anatomy-Sprains of the knee considered generally -Traumatic Hydrops-Sprains in which the internal lateral ligament is most concerned-Sprains in which the external lateral ligaments are most concerned-Separation of the body of the internal semilunar cartilage from the deep fibres of the internal lateral ligament-Schlatter's disease-Exostoses as cause of knee-sprain-Partial dislocation of the patella outwards.

\section{CHAPTER VI}

Internal Derangements of the Knee-Joint . . . 147

Displaced, detached, or torn internal semilunar cartilageAnatomy-Symptoms and clinical characteristics-Diagnosis-Causation-Treatment of first attack (immediate and subsequent); of recurrent or relapsing cases-Palliative treatment-Operative treatment-Essential preliminaries to operations-Displaced external semilunar cartilage-Hypertrophied synovial fringes. 


\section{CONTENTS}

\section{CHAPTER VII}

General Injuries to Muscles and Tendons . . . 194

Muscle sprain-Hernia of muscle-Displacement or dislocation of tendons-Rupture of muscle or tendon.

\section{CHAPTER VIII}

Injuries to Spectal Muscles and Tendons . . . 215

Ruptures of muscles and tendons-

Muscles of the Trunk-Rectus abdominis-Oblique muscles of the abdomen-Ilio-psoas muscle-Sacrolumbalis.

Of the Upper Extremity-Deltoid, 'cricket shoulder'Biceps cubiti-Triceps-Muscles of forearm.

Of the Lower Extremity-Rectus femoris-Quadriceps extensor-Hamstrings-Adductor longus-Adductor brevis and pectineus-Adductor magnus-Ligamentum patellæMuscles of the calf, ' lawn-tennis leg'-Tendo-Achillis.

\section{CHAPTER IX}

Massage, Movement, and Exercises in the Treatment of Sprains and Bruises . . . . . . .

Massage-Passive movements-Active voluntary movements-Forcible movements of stiff joints under an anæsthetic-Author's method for preventing atrophy in muscles of the thigh and leg-Gymnasium treatmentMovements and exercises against resistance.

\section{CHAPTER $\mathrm{X}$}

Special Methods of Treatment

Local applications-Bandages and strapping-Mechanical supports-Plasters for strapping-Application of high degrees of temperature-Bier's hyperæmic treatment-Injections of Fibrolysin-Mechano-therapy-Kataphoresis. 



\section{LIST OF ILLUSTRATIONS}

FIGURE

1. Fracture-sprain of ankle-joint .

2. Linear fracture of tibia in lower third as complication of sprained ankle ; illustrating 'sprain-with-fracture' .

3. Knee-joint permanently flexed and stiff as result of enforced movements and exercise while the semilunar cartilage remained displaced

4. Knee-joint fully distended with fluid as result of severe sprain . . . . . . . . . . 21

5. Photograph of knee-joint with elastic compression applied

6. Radiogram of fracture of left olecranon without displacement • . . . . . . . . . 39

7. A case of atrophy from disuse . . . . 44

8. Photograph of knee showing scar indicating author's line of incision for exploratory arthrotomy . . .

9. Chart from a case of exploratory arthrotomy for suspected fringes with adhesions

10. Photograph of old injury to knee-joint with atrophy of muscles of the thigh and hypertrophy of those of the calf

11. False joint at hip, result of faulty treatment of fracture of the neck of the femur by movement and exercises .

12. Radiogram of deformed ankle as the result of fracturesprain with separation of epiphysis of tibia and greenstick fracture of fibula.

13. Radiogram of false joint in the lower third of the humerus of ball-and-socket type, resulting from too early movements at the elbow in the treatment of a fractured arm

14. Radiogram of injury to wrist treated as sprain. Injury to epiphysis of radius resulting in defective growth .

15. Same case as Fig. 14 seen in profile . . . .

16. Radiogram of fracture of first phalanx of little finger with permanent deformity . . . . . . 
17. Radiogram showing ossifying myositis in extensor muscles of the thigh

18. Radiogram of same case at a later stage . . .

19. Radiogram of extensive ossifying myositis in extensor muscles of thigh, result of sprain at football, showing distinct lamination

20. Photograph of thigh with swelling on the extensor surface due to mass of ossifying myositis . . . .

21. Radiogram of lower third of arm showing myositis ossificans in brachialis anticus muscle as result of violent strain

22. Radiogram of knee-joint with partial detachment of a portion of the rim of the external femoral condyle .

23. Photograph of structures removed from a knee-joint by operation in which there was displacement of an internal semilunar cartilage of long standing with chronic synovitis . $\quad . \quad$..$\quad$. . . .

24. Radiogram of elbow showing fissured fracture of head of the radius as result of falling on extended arm . .

25. Radiogram of a fracture-sprain at the elbow first treated as a simple sprain . . . . . . . .

26. Radiogran of an impacted fracture of the olecranon; thought at first to be, and treated as, a simple sprain

27. Radiogram of oblique fracture through lower end of radius in which there was no displacement . . . .

28. Radiogram of a fracture of the tip of the styloid process of the ulna

29. Radiogram of impacted fracture of lower end of radius without displacement . . . . . . .

30. Radiogram of sprain-dislocation at the wrist in a child, showing separation of the radial epiphysis and tearing of the periosteum

31. Complicated sprain of wrist. Greenstick fracture of lower radius, and detachment of the tip of the styloid process of the ulna

32. Fracture-sprain of wrist. The scaphoid bone fractured into three pieces by blow while boxing . . $\quad$ • 95

33. Fracture of scaphoid of wrist into two pieces $\quad$. 96

34. Fracture of scaphoid of wrist (football accident) . $\quad 97$ 
35. Radiogram showing complete fracture of scaphoid bone of left wrist as result of a fall on to the hand . . 101

36. Radiograms of Bennett's fracture at the base of the 37. metacarpal bone of the thumb . . . . 103, 101 38. Linear fracture of the metacarpal bone of the thumb, without displacement . . . . . . . 105

39. Radiogram of fractured first phalanx of index finger . 107

40. Radiogram of greenstick fracture of the first phalanx of the index finger . . . . . . . . . 107

41. Radiogram of a greenstick fracture of the metacarpal bone of the second finger, showing well-marked callus as result of rubbing

42. Photograph of a sprained ankle, which had been incorrectly bandaged during treatment

43. Radiogram of sprain of ankle with fracture (linear) through the lower third of the tibia

44. Photograph of sprain of knee-joint taken four hours after the accident, showing full distension of the capsule .

45. Photograph of case of acute traumatic synovitis of kneejoint as result of displacement of the external semilunar cartilage taken two hours after the accident . .

46. Photograph of two knees with chronic thickening of the synovial membranes in a case of recurrent displacement of the semilunar cartilages . . . . . .

47. Radiogram of a case of Schlatter's disease . . . 137

48. Radiogram of a similar case . . . . . . . 139

49. Photograph of anterior half of a semilunar cartilage of the knee

50. Photograph of anterior half of a left internal semilunar cartilage of the knee . . . . . . .

51. Photograph of a drawing from a specimen of an internal semilunar cartilage, showing longitudinal tearing into three strips . . . . . . . . .

52. Photograph of a drawing made from the upper surface of the head of left tibia . . . . . . . 151

53.

54. Four stages in the author's method of reducing displaced

55. semilunar cartilages . . . . 164, 165, 166, 167

56.) 


\section{xviii}

57. Schemes to illustrate further the method of reduction .

58. Piece of bone detached from the edge of the patella; which had become rounded off and formed a loose body in the knee-joint

59. Block of hinge apparatus for the knee . . . . 174

60. Photograph of knees to show old transverse scars in the removal of semilunar cartilages

61.) The three stages in the author's operation for exploratory

62. arthrotomy and for removal of semilunar cartilages

63. from the knee . . . . . . 178, 179, 180

64. Charts representing the average temperature after arthro-

65. tomy of the knee-joint when no drainage is used . 18t, 185

66. Charts representing the same when drainage of the joint is

67. employed for twenty-four hours after operation . 186, 187

68. Long-standing displacement of left external semilunar cartilage of knee, showing atrophy of muscles of the thigh and hypertrophy of those of the calf, also flexion and fore-shortening of the limb .

69. Recent case of displaced external semilunar cartilage, with flexion and swelling of the left knee . . . 192

70. Photograph, showing hernia of muscle of thigh . . 196

71. Dislocation of peroneus longus tendon on to the external malleolus

72. Back view of a displacement of both the peroneus longus and brevis tendons from their groove behind the malleolus

73. Photograph, showing displacement of the tibialis posticus tendon on to the inner malleolus

74. Strapping applied for rupture of right rectus abdominis muscle

75. A. Strapping applied for sprained side (intercostal muscles). B. Strapping applied for sprain or rupture of the oblique muscles of the abdomen-'bowler's side'.

76. Rupture of the rectus femoris muscle, also old scar for removal of semilunar cartilage of the knee $\cdot$. third

77. Photograph of rupture of rectus femoris muscle in its lower 


\section{LIST OF ILLUSTRATIONS xix}

FIGURE

PAGE

78. Strapping applied for rupture of the hamstring muscles in the upper half of the thigh . . . . . 226

79. Long-standing case of rupture of the ligamentum patellæ on both sides with characteristic deformity $\cdot$. . 233

80. Radiogram of the left knee of the same case . . 234

81. Apparatus worn for rupture of both patellar ligaments to enable patient to walk $. \quad . \quad . \quad . \quad . \quad .235$

82. Strapping applied for 'tennis-leg', a rupture of fibres of the soleus or plantaris muscles . . . . . . 237

83. Ankle strapped for strain by author's plan . . . 256

84. Strapping of shoulder for contusion or sprain . . 257

85. Elbow-joint with elastic pressure applied for sprain with

effusion $. \quad . \quad . \quad . \quad . \quad . \quad . \quad . \quad .258$ 



\section{SPRAINS AND ALLIED INJURIES OF JOINTS}

\section{CHAPTER I}

\section{INTRODUCTORY}

THE subject of sprains is quite inadequately treated in the ordinary manuals of surgery. Modern methods of diagnosis have taught us much concerning the anatomical conditions underlying strains and sprains, and these injuries can only be adequately diagnosed and treated by their means. The present widely divergent views on the treatment of sprains is itself evidence of the want of exact knowledge concerning the pathological conditions present. On the one hand the bone-setter treats every sprain by massage and movement, while the usual custom among medical practitioners is to treat these conditions by rest, the joint being fixed in splints. Both these rule-of-thumb methods may do a great deal of harm. The bone-setter who manipulates a sprain complicated by fracture may displace the fragments, and by his unscientific movements cause an excessive amount of callus to be thrown out, with resulting permanent deformity of the joint. The consequence of putting every sprained joint at rest by fixing it in splints is often equally or even more disastrous. The resulting atrophy of the 
muscles moving the joint, and the formation of adhesions within it, produce not infrequently total disablement of a joint which, with proper treatment, might have recovered entirely. For the last eighteen years the writer has made a special study of these injuries, and has had unusual opportunities of treating a large number of such cases.

Concerning the Structure and Functions of Joints. Joints are constructed for movement. The bones which enter into their formation are covered with a thin layer of smooth, highly polished, hyaline cartilage. This enables them to move smoothly upon one another with a minimum of friction, and no jarring. They are tensely and firmly bound together by the ligaments and tendons which surround them. The capsule which envelops the ends of the bones is invariably continuous with the periosteum, and securely encloses them in what is but a potential space. The capsular ligament is lined by the synovial membrane, which extends to the edges of the cartilaginous border. This membrane is polished, and lined on its visceral surface by endothelium, and serves to secrete a fluid which lubricates the inner surface of the joints. It is thrown into folds wherever it is not in contact with the articular surfaces, and is covered with villi, or papillae, which, in certain conditions of disease, are liable to thickening and hypertrophy. The interarticular ligaments are closely enveloped by it. It secretes a viscous fluid known as synovia, the chief constituent of which is mucin. The function of synovia is that of a lubricant. The bones, cartilages, ligaments, periosteum, synovial membrane, and, in 
fact, all the tissues which enter into the formation of a joint, are developed from a common embryonic origin, the mesoblast; hence, in certain cases of injury, and as a result of certain pathological states, they become interchangeable and take the place of one another, though to a limited extent.

The villi of the synovial membrane may develop cartilage, or even bone, when unduly stimulated, and the ligaments may undergo ossification. A healthy joint contains but a potential cavity. This becomes a real one if the capsule should be distended either with blood or serous effusion, in consequence of injury or disease. Such distension gives rise to stretching and elongation of the ligaments, allowing of greater movement between the bones, while the joint becomes weakened and unstable, and more liable to injury. The synovial cavity empties itself through stomata into the lymphatics, which pass through the capsule and enter the lymph spaces in the inter-muscular and fascial strata. Blood is freely supplied by the articular vessels. The nervous supply of a joint is generally the same as that for the muscles which act upon it, and for the skin over its surface. The importance of this interesting physiological relationship will again be referred to.

The strength of a particular joint depends upon-

(1) the arrangement of its bony surfaces one to the other ;

(2) the nature, strength, and disposition of its ligaments ;

(3) the muscles and tendons which surround it;

(4) its normal amount of mobility.

Some joints derive their stability from the accurate 
adaptation of the opposing bony surfaces to one another. The ball-and-socket arrangement of the hip, and the hinge of the elbow, are mainly responsible for the great strength of these articulations. It requires very great force to dissociate or undo the bony apposition, and dislocations are rare without being associated with serious ligamentous tearing or fracture of one or other bone.

On the other hand we may take as an example such an articulation as the shoulder, a joint quite devoid of any bony influence, and dependent entirely for its integrity upon the strength of its ligaments and the muscles enveloping and tendons passing over and around it. A very trifling amount of violence exerted unexpectedly, catches, so to speak, the muscles unawares and the bones in an unusual position, and is sufficient to produce a complete dislocation. The very range of movement enjoyed by the shoulder is its source of weakness; for what it gains in mobility it loses in stability. Between these two extremes is an intermediate group of joints, dependent, on the one hand, partly on the formation of their bony surfaces, and partly on the support afforded by the muscles and tendons that envelop or surround them. The knee, wrist, clavicular, tarsal, carpal, and many other smaller articulations, derive their strength mainly from the ligaments which firmly bind their several bones together. It is in this group that we meet for the most part with strains and sprains. When such a joint is subjected to external violence, whereby strain is put upon it, the brunt has to be borne by the ligaments. If the violence is slight, or the ligament 
a strong one, nothing more serious than an overstretching or strain results ; if, on the other hand, the violence is extreme, not only individual ligaments, but whole groups of ligaments may be completely torn across. This class is represented by those joints whose chief strength is derived from their ligaments.

Some joints possess inter-articular cartilages which, though technically ligaments, since they bind bones to bones, are a source of natural strength in an indirect way. The amount of gliding or similar movements allowed by these inter-articular structures serves often to turn off, as it were, the main strain into some other direction as soon as violence is brought to bear on them. While every variety of joint is liable to sprain, it may be stated generally that those joints dependent on the strength of their ligaments are those most prone to this form of injury. 


\section{CHAPTER II}

\section{THE GENERAL CONSIDERATION OF SPRAINS}

ALL classes of society are liable to sprains, the rich as well as the poor. Sprains are very frequently met with, and although they cannot be classed as accidents of a dangerous kind, they are nevertheless serious. Great numbers of persons are incapacitated from their daily calling, wage-earners as well as pleasure-seekers. There is, perhaps, no more fruitful field than the athletic. For some inexplicable reason, the importance due to such crippling ailments has been little considered by surgeons in the past. Such disablements, in the vast majority of cases, seem to have been relegated to the rule-of-thumb attentions of the bone-setter, a class of persons with little or no scientific training or interests.

At the present time, when the achievements of modern operative surgery everywhere resound, and new advances are made in different departments, in the scientific as well as the practical, these socalled 'little things' of minor surgery are apt to be neglected and considered as of subsidiary importance. Yet, if the wage-earning capacity of the nation is taken into consideration, the annual loss must be enormous, and under the new Workmen's Compensation Act the expenses of the employers of labour are not likely to be diminished. This latter consideration, perhaps more than any other, may 


\section{GENERAL CONSIDERATION OF SPRAINS 7}

in the future redirect the attention of surgeons to a branch of surgery which, to say the least of it, has been somewhat neglected. Already there are signs that the empiricism of the much-consulted and the much-in-request wiseacre or bone-setter, the person who invariably finds ' something out of place', 'a bone out of joint', and whose stock treatment is to 'move everything', is giving way slowly, though surely, to a more rational and, if one may be permitted to say so, scientific and discriminating régime. Nevertheless, there are still thousands of persons, men and women of education and culture, who resort to these unregistered, irresponsible practitioners, and consult them with a confidence that is truly surprising.

It surely behoves our profession to pause, consider, and make inquiries, as to whether means may not and should not be taken to remedy this state of things ; for not only is it an opprobrium to ourselves, but hundreds of people, owing to improper rule-ofthumb and irresponsible treatment, are permanently incapacitated and crippled. The victims, unprotected by the legislature of the country, often find out their mistake when the time for remedial measures is past, with frequently a legacy in the shape of a damaged limb for the rest of their lives.

Definition. When the movements in a joint are carried beyond their normal and physiological limitations, owing to some wrenching or twisting form of violence, and the soft structures which bind the component bones together are overstretched or torn across, such an injury is said loosely to be a strain or sprain. The term strain in present-day usage 


\section{GENERAL CONSIDERATION OF SPRAINS}

implies the injury of less degree and is unaccompanied by swelling. At one time the classical conception of a sprain was 'a wrench or strain resulting in stretching or laceration of the soft parts without external wound'. Such a definition at the present time requires some modification, for although in the main it is an accurate description of the anatomy of the affection, yet there is a certain proportion of cases which, formerly classified with sprains, would at the present time be more properly grouped with fractures. For example, in the injuries to the larger joints it is not an uncommon experience to find small fragments of bone detached with the ligaments where they have been torn from their periosteal attachments. Again, in the smaller articulations, as those of the wrist, hand, and foot, the small bones may be fissured, chipped, or even crushed; so also small epiphyses are sometimes dragged away by the tendons in muscular sprains.

The use of the $\mathrm{X}$-rays as a means of diagnosis has revealed many such bony defects where formerly they might not have been suspected. For clinical purposes, however, these injuries may still be regarded as sprains, for the symptoms of fracture are of minor importance as compared with those of sprain, which usually greatly predominate. Their true pathological significance must never be lost sight of nevertheless, for the existence of a fracture, however slight, materially changes the treatment. To this, reference will be made later, when the question of treatment is under consideration.

Classification. For the purposes of classification, sprains may be divided into two large classes. 
1. Articular, or those affecting the joints themselves ;

2. Muscular, concerned with the overstretching, dislocation, or rupture of muscles and their tendons.

1. Articular Sprains, for clinical purposes, may be arranged into two divisions or classes: (a) simple, (b) complicated.

(a) Simple Sprain. By the term 'simple sprain' are here meant only those cases where, as the result of some external violence, the soft parts are overstretched or torn across, and in which no gross lesion of the bone is to be found.

In the simplest form of sprain, that technically described as strain, the soft parts are merely stretched beyond their usual capacity, and there is no true laceration. No swelling is to be found, and the symptoms are only those of pain and stiffness with some loss of function.

In the more severe forms of simple sprains, in which there has been more or less tearing, as well as stretching of the ligaments and other structures of the joint, in addition to pain and loss of power, we get swelling. When the swelling follows immediately after an injury it is due to hæmorrhage. Arteries as well as veins are torn across, and pour their contents into the tissues around. Hæmorrhage is sometimes, as we shall see when dealing with muscular sprains, of large amount and serious consequence. The bleeding usually ceases as the tension in the parts increases, but it sometimes happens that, when the injured blood-vessel is a comparatively large and important one, and when the tissues into which it drains are loose and distensible (as may be 


\section{GENERAL CONSIDERATION OF SPRAINS}

met with in some of the fascial planes of the body), it may continue for a considerable time after the injury, and give rise to extensive swelling. Hæmatomata result from the blood clotting in the tissues.

When the swelling in a joint comes on some hours or even days later, it is due to serous effusion, and is known as 'deferred swelling'. This varies considerably in amount in different cases, depending upon a variety of causes such as the nature and severity of the injury, the health and idiosyncrasy of the individual, and sometimes even to improper treatment early in the case. It is regarded by some authorities as inflammatory in nature; by others, as a necessary concomitant, and as possessing more of a physiological than a pathological significance, and even of being concerned in the process of repair.

There is something to be said for both views. That it is of pathological significance must be admitted, since it is the result of injury and is only present to any appreciable extent when diseased conditions coexist.

(b) Complicated Sprains.

1. Sprains become complicated when there are gross injuries to the bone or its periosteum, as when portions of bone or epiphyses are detached, and when the bones are fissured or even crushed. Such a lesion is now loosely designated a sprain-fracture, or fracturesprain. It should, however, be clearly understood that there is a marked difference between a fracturesprain and a sprain with fracture (vide p. 38 et seq., and Figs. 1, 2).

When the sprain is complicated by an injury to a bone entering directly into the joint, or to a neigh- 


\section{GENERAL CONSIDERATION OF SPRAINS 11}

bouring epiphysis, it is called a fracture-sprain or sprain-fracture. If in addition to the sprain of a joint there is a fracture of one of the bones in its immediate neighbourhood but not involving the

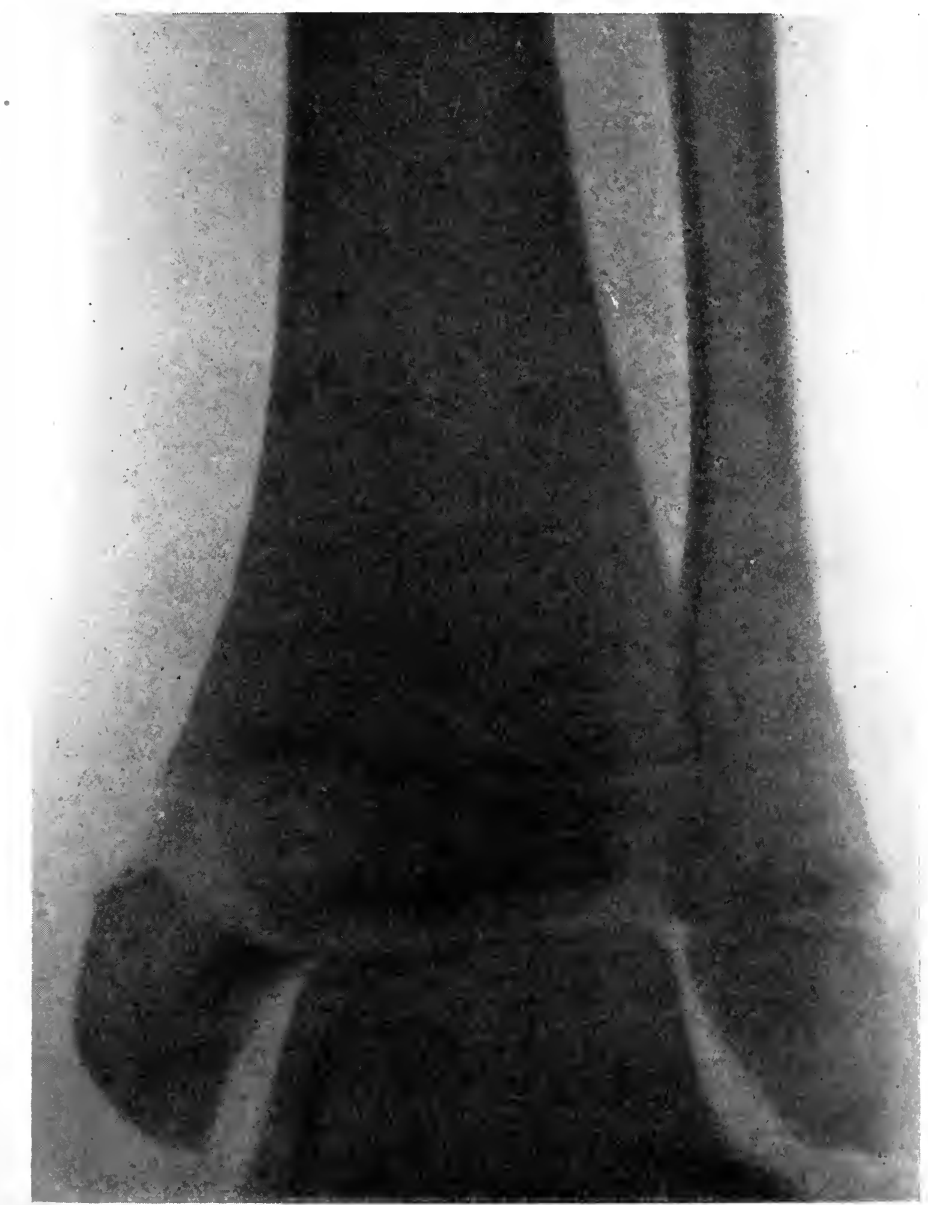

Fig. 1. Illustrates a fracture-sprain of the ankle. The radiogram was taken from the joint of a boy aged 14, and shows a separation through the internal malleolus. The lower epiphysis of the tibia including the malleolus is normally developed in a single piece. 


\section{GENERAL CONSIDERATION OF SPRAINS}

joint itself, the expression sprain with fracture may be used. Figs. 1 and 2 illustrate these definitions, and should be compared.

2. In some joints, such as the knee, temporomaxillary, and clavicular, in which inter-articular cartilages are to be found, it is no uncommon circumstance for these structures to become partially detached or torn across and displaced, either inwards towards the cavity, or out of the joint. Though technically a semiluxation or partial dislocation, since the symptoms of sprain so greatly predominate, it has been customary to regard such an accident as a sprain, and to treat it as such. This, from analogy, may be styled a sprain-dislocation.

3. In a few cases the nerves suffer injury and complicate matters (vide pp. 35-37). They may be overstretched, resulting in temporary paresis, or a sensation of numbness may be experienced in some branch far removed from the injured joint. Pain is generally the chief symptom when nerves are pressed upon by inflammatory exudation, or overstretched in some part of their course. It may be localised to the seat of injury, or radiate along a nerve-trunk around the body or into the extremities. If not detected early, or if improperly treated, this complication gives rise to serious and prolonged crippling. A form of chronic atrophic neuritis may supervene, and atrophy of the muscles supplied by the special nerve, of a peculiar and lasting kind, result. Unless carefully sought for at the time, such lesions are apt to be overlooked by the surgeon, for patients seldom attach importance to sensations referred to parts of the body distantly removed from the main injury; 


\section{GENERAL CONSIDERATION OF SPRAINS 13}

nor is it difficult to miss some small area of numbness or altered sensation in the skin. When large or important nerves, such as the sciatic, or branches of the brachial plexus are concerned, for obvious

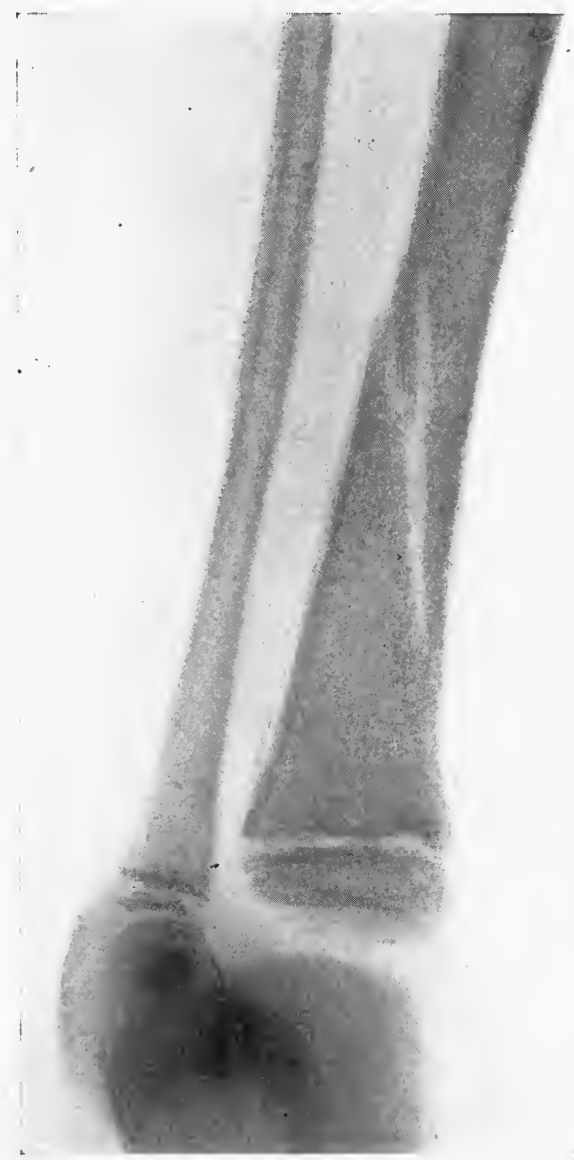

Fig. 2. To illustrate the author's 'sprain-with-fracture'. Note the linear split through the tibia in its lower third without displacement, from a boy who walked into the hospital complaining of having sprained his ankle. The fracture does not extend into the joint. Radiogram taken two days after the accident. 


\section{GENERAL CONSIDERATION OF SPRAINS}

reasons the symptoms are more readily noticed. It is therefore a wise precaution in all severe sprains for the surgeon to search for possible nerve lesions.

4. Sprains with very large hæmatomata should be regarded as complicated sprains, for they may require even operative measures for their successful treatment. Large collections of effused blood are met with not infrequently as the result of sprains and lacerations of muscles; especially in situations where there is little resistance offered to the extravasated blood, as afforded by the loose planes of cellular tissue between groups of muscles, as those of the thigh and abdomen, and the sheath of the psoas and iliacus.

Diagnosis. The importance of an early diagnosis in all cases cannot be too strongly advocated, nor can this advice be too often repeated. It may not always be possible to make an exact or complete diagnosis, but to get as near to the truth as possible should be the first aim. The difficulty or otherwise in arriving at a correct diagnosis will depend largely upon the period at which the surgeon is called to see the case. If he is fortunate enough to be consulted before much swelling has taken place, his task may be comparatively simple; on the other hand, when much material has had time to be poured out into the surrounding tissues and increased by subsequent inflammation, his difficulties may be almost insurmountable. It is always a sound plan, when in doubt, to completely anæsthetize the patient. This should be done in all cases where there is no potent contra-indication such as the presence of advanced organic disease or insuperable 
objection by the patient. The joint should then be put through all its movements. In doing this the synovial membrane will be put fully on the stretch, and any folds which may have been made will be unfolded, the curling in of torn fragments of the capsule-a circumstance of no infrequent occurrence - prevented, and any undue fixity or abnormal mobility of the joint itself noted.

When the inter-articular fibro-cartilage has been torn or displaced, the movements, at first limited and incomplete, may be at once restored by a free and painless manipulation.

Crepitus may or may not be elicited, and it is of importance to direct attention to this important symptom. The absence of or failure in detecting crepitation does not at once dispose of the possibility of fracture any more than does its presence give positive proof of its existence. By this is meant that there are cases of fracture in which crepitation cannot by any means be detected, such as in fissured injuries of bone, while it is often distinct and quite easily palpable in joints the subject of osteo-arthritis, or where sticky material is effused into tendon-sheaths in the neighbourhood of injured joints, and where no fracture exists. Where there is no fracture, unusual mobility will give the observer a fair estimate of the amount of damage sustained by the ligaments as a whole, as well as by the particular ligament, or group of ligaments, which have borne the brunt of the incidence of the violence. Having ascertained all that can be learned by manipulation with the aid of a general anæsthetic, if it be possible or practicable, 


\section{GENERAL CONSIDERATION OF SPRAINS}

the limb or part should be examined by means of the $\mathrm{X}$-rays, under the management of a competent person, and let us say competent advisedly, for the correct interpretation of a radiogram requires considerable surgical knowledge and practice. The $\mathrm{X}$-rays will settle once and for all the existence or otherwise of a fracture, a point of incalculable service, as will be noted when treatment is considered. The X-ray photograph should be taken from different points of view, or it may fail to show a fracture.

A sprain complicated with injury to a bone or displaced, torn, or otherwise detached inter-articular cartilage, or with gross nerve lesion, or extensive extravasation, will require special and careful handling.

Prognosis. The immediate prognosis in the case of simple sprains and strains is good, but in complicated cases some caution must be exercised.

Sprain-fractures, when not detected and when treated by ordinary means like simple sprains, may lead to prolonged stiffness and much unnecessary suffering. So in cases of gross nerve lesion, continued pain and muscle atrophy, even of a permanent kind, may supervene, as well as actual local paresis or palsy. Disorganization of a knee-joint has been known to result from enforced exercises in a case of undetected displaced semilunar cartilage (Fig. 3). These extreme cases, however, are rare.

Certain remote contingencies arise which are quite beyond the control of the surgeon, such as osteoarthritis, myositis ossificans, palsy, tumour or tuberculosis, loose bodies in the joint, as well as others, such as deformity, which can be, for the most part, 


\section{GENERAL CONSIDERATION OF SPRAINS 17}

prevented. These remote and permanent consequences will be referred to again when dealing with the sequelæ of sprains. The question of prognosis is becoming day by day a more and more practical

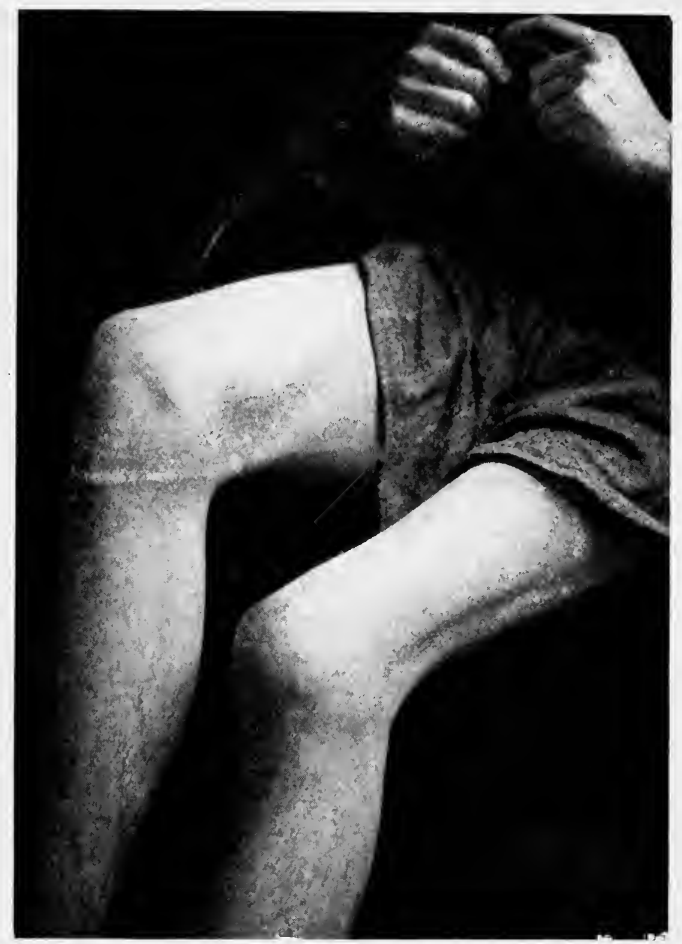

FIG. 3. Photograph of knees. The left shows permanent flexion and stiffness, result of unreduced semilunar cartilage. Owing to mistaken diagnosis, case had been treated at a gymnasium for many months with movements, exercises, and massage. Patient now quite lame. Correct diagnosis had not been made in the first instance, as he was treated by a bone-setter.

and important one. The new Workmen's Compensation Act makes it almost imperative that clinicians should be able to give a reliable opinion in cases of injury. 


\section{GENERAL CONSIDERATION OF SPRAINS}

Treatment. Simple sprains, as distinguished from sprains complicated with lesions of bone or nerve, are of two classes : those in which pain, stiffness, and loss of function are the only symptoms, and those in which there is swelling in addition. For the former class the term 'strain' is used.

Simple strains are for the most part limited to strains about joints and to the muscles of the back and neck, and the short flat muscles of the trunk. They are not a large class. Those suffering from strains seek assistance mainly for the relief of pain, which may be intense. Absolute rest to the injured part is the first indication where pain is concerned, and this may best be attained by the aid of strapping. Various materials are used for this purpose. The best are those which give a more or less resilient or elastic support, such as wash-leather strapping and leucoplast. Mead's or Leslie's plaster may be used, but do not answer the purpose so well ; they are, however, much cheaper. Perhaps, all things considered, Seabury's adhesive perforated rubber plaster is the most universally applicable; it is strong, allows of evaporation, and is not quite as irritating as some other forms; it has a further advantage of being comparatively cheap, and may be had in convenient sizes. All strapping must be carefully and evenly applied, so as to ensure perfect rest to the parts concerned. As soon as the pain ceases, or is materially lessened, gentle stroking massage may be employed twice or oftener in a day. Massage, in some instances, will further reduce pain, but slight voluntary movements should be encouraged from the very first, as thereby stiffness, the other 
symptom of strain, is readily and speedily overcome, and the healthy physiological nutrition of the joint or muscle promoted, with complete restoration to function.

Sprains with 'immediate swelling'. Bleeding into the tissues follows almost immediately upon the injury, the blood being poured out from the ruptured vessels. The swelling is usually established before the surgeon is called to the case. If however the case is seen early, an attempt should be made to arrest or check further hæmorrhage. Various means have been employed to effect this, and while one practitioner will employ cold as a routine, another will, with equal confidence, use nothing but warm applications. The writer has for years entirely abandoned the use of either cold or hot applications -neither is, in his opinion, sufficiently efficacious. Cold, to be of any real value, must be continuous and intense, and this is not without danger to persons suffering with organic (cardiac or renal) disease. Cases are on record where extensive sloughing has followed upon the continued use of cold in persons suffering from albuminuria, glycosuria and other debilitating affections. Warm applications are better tolerated, and even liked, by patients, but they would only seem to encourage rather than check the extravasation of blood. Any application of heat that would be sufficient to reflexly produce contraction and retraction of the torn ends of deeply situated blood-vessels, would not be borne with any comfort, nor would this be safe as a routine proceeding. The skin of some individuals is peculiarly liable to be damaged by high degrees of temperature, 


\section{GENERAL CONSIDERATION OF SPRAINS}

whether the heat is dry or moist. The most efficacious, and at the same time the most readily applied remedy is firm elastic pressure. Elastic pressure is suitable for all cases of extravasation, whether of little or great amount, whether immediate or deferred. It has the advantages of being absolutely safe, easily applied, and of universal application ; it ensures perfect rest, and, if employed immediately or soon after an injury, decidedly diminishes or completely prevents those secondary effusions so apt to follow. Furthermore, it promotes, to a marked degree, the rapid absorption of extravasated material, the next great desideratum in the treatment of all kinds of sprains. Thus, by ensuring rest, checking or limiting immediate hæmorrhage, preventing or at any rate diminishing secondary effusion, promoting the absorption of all extravasated material, it serves the double purpose of meeting the two first and all-important desiderata-those of checking hæmorrhage and removing extravasations. When properly applied, it takes the place of a splint and ensures rest; its mode of action may be observed in the treatment of contusions. The principle is simple : in applying equable elastic pressure of a higher degree than that of the blood pressure, the escape of material from the bloodvessel is mechanically prevented, and this pressure, exerted on the fluid materials that have already escaped into the tissues, being greater than that of the blood pressure in the vessels themselves, it happens that a process of absorption is soon initiated and the swelling is observed to subside apace. The same principle is at work in relation to the serous 


\section{GENERAL CONSIDERATION OF SPRAINS 21}

effusion which is almost invariably poured out secondarily into the synovial cavities of the larger joints. It is maintained that if elastic pressure can be properly and immediately applied to an injured joint, secondary effusions are unusual, and when such

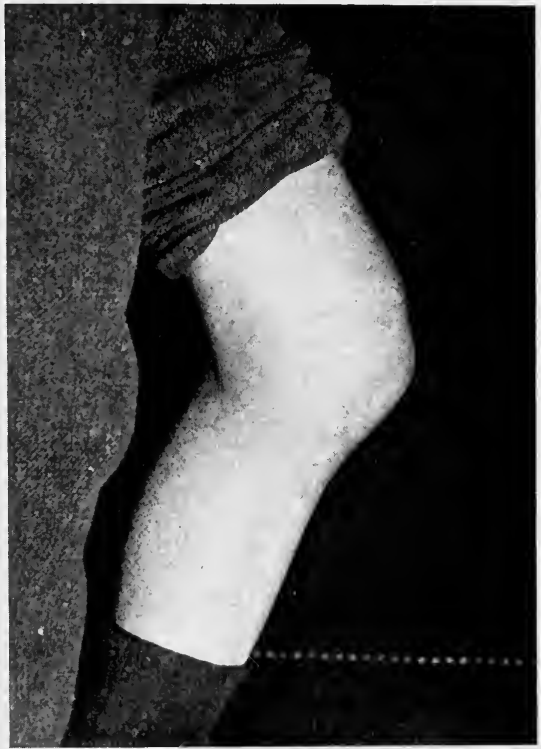

Fig. 4. Photograph of knee-joint in profile, taken two hours after severe sprain.

effusions do take place they do so to a more limited extent than when other remedial measures are adopted.

Elastic pressure may be used for any extravasation or effusion, whether localised or diffused, whether of rapid or more chronic formation. It matters not whether the material is confined within the capsule of the joint, or merely loosely collected in the inter- 


\section{GENERAL CONSIDERATION OF SPRAINS}

muscular and cellular planes of the trunk or extremities. The action is the same; its service invariably efficient. If a joint such as the knee be taken as an illustration, and if we suppose that, as the result of a twist, its capsule has become fully distended with effusion, and that most of the swelling took place immediately after the accident and continued to gradually increase for some hours afterwards, it will be found that such a knee assumes the posture of slight flexion (Fig. 4). This is a position which, from the nature of things, permits of the greatest relaxation of the muscles around the joint, and allows to the distended capsule its fullest capacity, and in this way best assures relief to the sufferer.

This is nature's first attempt at procuring rest, our first requirement in treatment; to extend such a joint and fix it in the straight position on a splint, as is so often recommended and practised, would not only violate this principle of securing rest, but render the patient exceedingly restless and uncomfortable. On the contrary, the limb should be left in the position that it naturally assumes, which is always that which is most comfortable to the patient. When the knee is partially bent, not only are the muscles relaxed, but the surrounding ligaments are also slackened; when, on the other hand, it is extended, these tissues are tightened up and exert pressure on the distended capsule, thereby tending to increase the pain and discomfort.

Method of applying Elastic Pressure. To the slightly flexed limb thick layers of cotton-wool are adapted. They are either rolled around the joint, or cut into shapes and so applied that a complete 
coating of at least one inch of thickness is attained. In length, the material should extend for at least two inches above and below the outside limitations of the capsular ligament. Gamgee-tissue, though more expensive, answers the purpose admirably ; it may be cut with greater nicety and exactitude than is ordinary cotton-wool, and so more easily adapted to the varying requirements. Over this thickness of material a strong roller bandage is tightly applied;

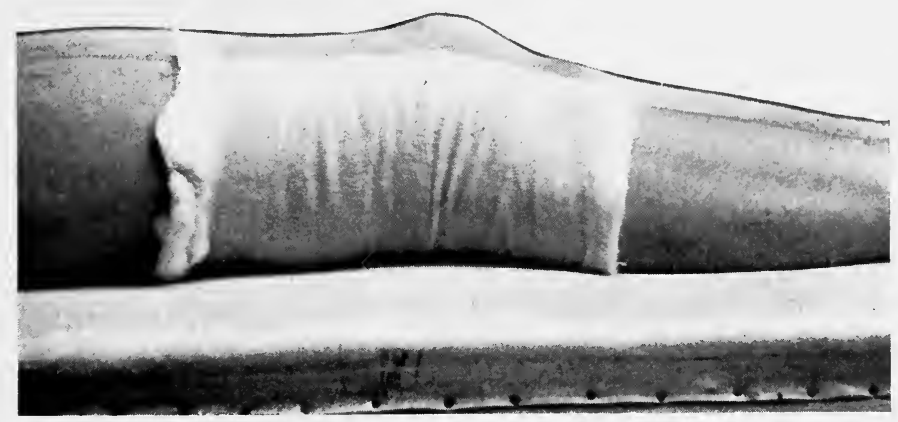

Fig. 5. Photograph of knee with elastic pressure applied for sprain. Author's plan. The knee is here represented as being too straight, it should be more flexed.

an inelastic yet resilient material, such as a domette or soft flannel bandage, is all that is necessary. Stout calico or crêpe-velpeau bandages are less useful, the former variety being a little $t \rho^{\circ}$ stiff, while the latter too soon lose their elasticity and become overstretched and unsuitable.

The bandage is then drawn as tightly as possible over the wadding so as to compress it to its utmost capacity, then fixed, to maintain it in this position. Great care must be taken to avoid creases in the wool, and to see that it is smoothly and equally 


\section{GENERAL CONSIDERATION OF SPRAINS}

applied, otherwise the pressure will not be evenly distributed. Four inches is a useful breadth for a bandage when applied to the trunk or lower extremity; a narrower one is more suitable for the upper limb. The photograph (Fig. 5) represents a knee-joint with elastic compression applied.

It is of great advantage, whenever possible, to remove the bandage about six hours after its first application, and to thoroughly re-examine the joint. Points of diagnostic significance are not infrequently lost sight of when making the first examination. By this time, in many cases, tension will have already become less. The patient, finding his pain considerably lessened, is usually less alarmed, and allows the surgeon more manipulative access to his injury. It may be assumed that whilst the first application of the elastic pressure mainly checks extravasation, the second and subsequent ones promote its absorption. It is well for the first three or four days, when practicable, to remove the bandage twice in the twentyfour hours. The surgeon will in this way be enabled to judge of the rapidity or otherwise of the process of absorption, while the intermittent removal and re-application of the bandage, by its influence on the local circulation, will directly assist in the treatment and hasten recovery, a fact of no little importance. As soon as the bandage is removed, the part that has been under compression becomes flushed with a new supply of arterial blood richly oxygenated, whilst, on its re-application the veins and lymphatics are once more compressed and their contents thereby emptied into the general blood-stream. This to-andfro flushing and depletion will be seen to be most 
beneficial, both in improving the nutrition and assisting absorption. It has been noticed, whether this is a true explanation or not, that swellings are more rapidly reduced in cases where the pressure has been intermittently applied than in those where it has been allowed to remain constant, and this is truer and more certain when the method has been adopted during the early stages of treatment. In most cases the effusion will disappear in about four days, but such a happy result depends largely upon the time at which treatment is commenced, and upon the care and time that the surgeon is able to bestow in carrying out the details.

The writer seldom uses massage in his practice until all or most of the effusion has disappeared. All that massage can effect, elastic pressure will, if properly and intelligently used. Where there are large hæmatomata in the tissues, or there is reason to suspect the presence of much clotted blood in a joint, the process of absorption must be expected to take longer. It is in these particular cases that the later use of massage, combined with elastic pressure, is most valuable. It has been said that elastic pressure is painful, that it produces numbness, that it is not well borne and for this reason should not be recommended. A word of warning seems necessary relative to this belief. Unless the operator applies a sufficient thickness of wadding, and unless the bandage is so applied as not to come within at least one inch of the upper or lower limits of the encircling wadding, painful constriction due to strangulation may result and cause discomfort. Pressure, when properly applied, is never uncomfortable; it is 


\section{GENERAL CONSIDERATION $N^{*}$ OF SPRAINS}

rather the reverse. A strong elastic rubber bandage should never be used. It is hot, allows of little or no evaporation, is too powerful, causes too much compression, and is often most uncomfortable. Rigid splints and fixed apparatus, whether made of wood, plaster, or other material, should never be employed, even in complicated cases such as sprain-fracture.

The primary object in the treatment of an injured joint is to restore its mobility in the shortest time. Everything should be done to prevent the formation of adhesions within or around it-weak, stiff, and painful joints result usually from the presence of adhesions. Much wasting of the surrounding muscles is apt to take place in a joint that is fixed in a splint. This is a serious complication, and must be prevented by all means. When the fluid effusion has been materially reduced, or for the "most part removed, and the torn ends of the bleeding. vessels, have had time to be effectually sealed off, then, and not until then, should masssge be employed. It is not usual to begin massage until at least seventy-two hours have elapsed. At first, gentle stroking massage should be tried for a few minutes, and then the bandage re-applied. If at the next inspection the fluid is still further diminished, and there is no heat in the joint, friction massage should be combined with stroking movements. This serves to break up the coagula and further disperse the absorbable elements of the clot into the surrounding tissues, and to promote more rapid absorption.

Active voluntary movements, though at first slight, may be allowed from the very beginning, as soon as the bandages are removed, as they are of 
primary importance in preventing adhesions and muscle-wasting, and of maintaining the proper nutrition of the structures of the articulation. They relieve rather than cause pain in most instances; they should be permitted early in the case only in those muscles that have escaped injury, and their range should be limited.

Passive movements are useful in a mechanical way, but possess little or no physiological merit. By stretching out the synovial membranes and capsular ligaments, they aid in restoring to their natural position fibres that are curled inwards and likely to form new and faulty attachments. Sometimes they occasion pain, but they are not to be compared with active or voluntary movements as regards their influence upon nutrition. The normal nerve-impulse, transmitted to the region of the joint through the muscles, will do more than any mechanical means, such as massage and passive movements, to restore physiological action and maintain the processes of repair. This fact should be steadily kept in view in dealing with other injured tissues of the body.

When a distinct interval of hours and even days occurs between the time of injury and the incidence of swelling, and in those cases where there is a recrudescence of symptoms after a period of apparent subsidence, it is probably due to secondary inflammatory changes. The treatment will depend upon whether or not there are present other signs of inflammation, such as pain on local pressure, heat, and increased tension.

In the absence of these acute symptoms, massage, 


\section{GENERAL CONSIDERATION OF SPRAINS}

or even movements both passive and active, may be persevered with. Especially useful are the gradually increased voluntary movements and exercises assisted by elastic pressure. If, on the other hand, there is pain, accompanied by a distinct rise in the surface temperature of the part, whether in the case of a joint or other region, all movements and massage should cease at once, and reliance be placed entirely upon the use of elastic pressure. The amount of pressure must be carefully regulated according to the degree of comfort or otherwise that it affords. A crêpe-velpeau bandage carefully applied and adjusted over a fair thickness of gamgee-tissue will not only give sufficient rest to an inflamed joint, but will allow it to assume that degree of flexion which is at the same time the most comfortable. It is in this particular class of cases that a fixed and rigid apparatus is most baneful; the limb often,from the method of its fixation in an extended position, becomes uncomfortable. The discomfort complained of leads the surgeon, in many instances at any rate, to the conclusion that he has to deal with a joint in which there is tension, or possibly even one with a progressive inflammation. Putting this interpretation upon the matter, he replaces the splintings and retains them in situ for an indefinite period, with the result that adhesions form, muscles waste, and a painful, stiff, and crippled joint remains.

These relapsing cases, if the term is used strictly in this special sense, are not infrequently associated with and dependent upon the health of the individual. Persons of a gouty, rheumatic, tubercular, or even syphilitic tendency, are subject to effusions in their 
joints, the injury being merely the localising cause. Nor must hæmophilia be forgotten. It becomes of the greatest importance, therefore, to make careful inquiry into the histories of all recurrent or relapsing cases, to search diligently and carefully for any other clue which may be corroborative of a suspicion as to constitutional taint. In a large percentage of cases such taint will readily be found, a fact which will greatly influence our views of the local treatment.

In rheumatic persons pain without effusion is often met with. The following case may be used as an illustration :

An undergraduate, thrown from his horse while hunting, fell heavily upon his right side, jarring the right hip-joint. He was able to continue hunting for a short run, when he felt his hip-joint becoming increasingly stiff. Returning to college he had a very hot bath, went to bed, and sent for assistance. A careful examination with an anæsthetic was considered advisable and immediately made. No fracture or dislocation was discovered. Treated as a sprain, the joint rapidly improved, and the patient was, able to resume gentle riding exercise fourteen days later. For the first ride or two he suffered no inconvenience whatever, and believed that he had completely recovered. He was then pressed by his friends into taking part in a football match, played in his accustomed place and thoroughly enjoyed the game. That evening the joint became once more stiff and exceedingly painful. A general anæsthetic was administered to allow of free manipulations of the joint as an aid to an exact diagnosis, and in addition the Roentgen rays were utilized. Nothing of the nature of a fissure could be detected in the bones of the pelvis or thigh. As the pain became not only more severe but almost constant, and having no reason to suspect either a gouty or syphilitic taint, since he was an exceptionally healthy and clean living man, the patient 


\section{GENERAL CONSIDERATION OF SPRAINS}

was put under the influence of the salicylates and aspirin in large doses. The next morning, and as soon as he had taken as much as $\mathbf{4 0}$ grains of salicine and $\mathbf{3 0}$ of aspirin, he stated that he 'was cured'. After a few days he ceased to take any medicine and resumed his riding exercises. Again the symptoms recurred, and this time there was definite effusion into the hip-joint. The anti-rheumatic remedies once more relieved his pain, though the effusion lasted for about a week. For months off and on this patient suffered from similar relapses whenever he over-exercised himself, and it was not until he had been prevailed upon to visit one of the well-known spas and to undergo a definite course of treatment that he finally lost all constitutional symptoms. This is but a type of innumerable instances of local injuries being influenced and exaggerated by some constitutional defect.

When there is a gouty history it is the usual custom to exhibit the iodides internally, and sometimes even colchicum, while at the same time local remedies are applied. Vasogen-iodine is an excellent local application; it produces but little irritation and is a convenient substance for 'rubbing in'. With syphilitic subjects small doses of mercury prescribed in pill are to be preferred to the continued administration of the iodides.

The treatment of hæmophilia is absolute rest coupled with the internal administration of calcium lactate. Ergot would seem to exert but little influence. Fortunately, these cases are extremely rare; nevertheless, the surgeon must not lose sight of them.

Tuberculosis as a sequela and complication will be referred to later. A large, superficially situated hæmatoma, especially if it is some distance from a 


\section{GENERAL CONSIDERATION OF SPRAINS 31}

joint, is best treated by a free incision, removal of all clots, and immediate suture. With aseptic methods drainage is not only unnecessary but usually inadvisable. Elastic pressure may with advantage be applied over the absorbable antiseptic dressing. When the skin wound is healed, which is usual in about ten days or earlier, and when the stitches have all been removed, massage may be begun. The scar should be avoided during the manipulation, and the movements confined for the most part to the peripheral regions at first. Extensive collections of blood are to be met with in the loose parts and spaces of the body, as in the upper thigh and buttock, in the axilla, sheaths of the rectus abdominis, and psoas and iliacus muscles. Such large collections are usually the result of arterial bleeding, and are found only in situations where a vessel of fair size may be torn. When muscles are torn across, the bleeding is mostly venous, on rarer occasions arterial. A branch of the deep epigastric artery is sometimes wounded when the rectus abdominis muscle is torn across as the result of violent muscular strain, or from blows upon the lower abdomen.

\section{Tension in Joints after Sprains}

The 'tension in joints' of writers is the condition which sometimes follows immediately an injury to a joint, or occurs at some later period, it may be hours, days, or even weeks after. In such a condition we find the capsule of the joint distended, it may be to its fullest capacity, by a fluid effusion. When this is early in its appearance, immediate, in 


\section{GENERAL CONSIDERATION OF SPRAINS}

fact, it must be regarded as the result of hæmorrhage ; when coming on gradually, creeping on, as it were, it is the consequence of secondary and inflammatory processes. Inflammation may result from renewed local injury or from any of the constitutional states already enumerated, such as gout or rheumatism (p. 28). Local heat is invariably a symptom of tension; it may be marked and easily to be detected with the aid of the hand, or so slight as to be hardly discernible by touch. The more rapid the incidence of the swelling, as a rule, the more distinct are the evidences of heat locally. Pain, often constant, readily increased by any movement, whether passive or active, is complained of. This is often severe, so severe, indeed, as to give rise to symptoms of constitutional disturbance such as sleeplessness, loss of appetite, and general irritability. In the most severe cases, where even a bandage is tolerated with difficulty, one of two lines of local treatment is adopted. Where there is no important constitutional contraindication existing, such as marked or severe cardiac or renal disease (glycosuria or chronic albuminuria), and where there is no suspicion of hæmophilia, much relief from pain will result if, with the aid of an anæsthetic, the joint is aspirated. All that is required in the majority of cases is that an ounce or at most two ounces of serum be withdrawn from the cavity. The relief afforded is as quickly experienced as is the cure of toothache when the tooth is extracted. There is never any necessity for drawing off all, or even most, of the contents of the joint; indeed, it has been found that recurrences are more usual after the full removal than after partial. The require- 
ments can be sufficiently met if only as much effusion is withdrawn as will diminish the tension and relieve the pain so that elastic pressure may be applied and tolerated with some degree of comfort. Not only does aspiration relieve pain, but it initiates the processes of absorption. The blood-vessels and lymphatics around a joint that is completely distended are overstretched or mechanically blocked, so that either absorption ceases altogether or goes on at a disadvantage. The withdrawal of the fluid with the relief of tension in the joint, assisted by the elastic compression applied to the tissues around the joint, give such assistance as is necessary for displacing the fluid towards the general circulation. In this way the continual absorption of extravasated materials is insured. Such is the theory upon which the practice is based.

When a patient objects to aspiration or any other form of operation, or when there are other contraindications, the symptoms are often materially and readily relieved by submitting the joint to dry heat in a bath. This may be carried out by means of a radiant heat apparatus. In a certain proportion of cases, although not a large one, this method is useful, and may even work like a charm; yet, occasionally, it must be admitted, this plan of treatment is absolutely useless and fails entirely. When dry heat is unobtainable, or where it has been given a fair trial and has not come up to expectations, hot fomentations with laudanum or lead lotion may have a soothing effect. As soon as it is noticed that the tension is diminishing, either of itself or as the result of treatment, elastic pressure, followed by or 


\section{GENERAL CONSIDERATION OF SPRAINS}

combined "with massage, may be commenced and continued until all or most of the fluid has disappeared. Movements, voluntary and passive, must be withheld until tension has quite or almost gone, and so long as any pain or local temperature remain. In recurrent cases, and in those in which the integrity of the joint is at stake owing to chronic distension of the capsule, and where considerable wasting of the muscles exists and seems to be increasing, arthrotomy and digital exploration of the joint itself may be necessary and even advisable. No open operation should, however, be Hghtly undertaken, especially where the knee-joint is concerned. The writer has on several occasions found that the opening of a knee-joint is the only satisfactory means of dealing with faulty adhesions within its capsule. Arthrotomy and temporary drainage are only to be recommended as a last resource where other means have failed, and when it is considered that the joint must otherwise become permanently damaged. The surgeon must be, moreover, one who is fully alive to the risks of opening the knee-joint and one who has the fullest confidence in the methods of his surgical technique, for unless absolute cleanliness and thorough asepsis can be guaranteed, arthrotomy should never be practised. More will be written in a later chapter as to the details of arthrotomy as advocated and practised by the writer (vide p. 177 et seq.). 


\section{SPRAINS COMPliCated With INJURy to the Nerves}

Of the complications of sprains this is one of the most important, and requires special attention as regards diagnosis and treatment. In the laceration of muscles, as well as in the overstretching of the ligaments of joints, branches of nerves are liable to injury. Even a gross lesion in a nerve may escape the notice of the surgeon when making his first examination, unless he carefully looks for it. It is often only when imperfect recovery has taken place, and when the reasons for this are sought, that a clinician begins to suspect that there may have been some injury to a nerve. When pain radiates along the branches of a large trunk like the great sciatic, or where there is marked and distinct paralysis, the observer's attention is at once arrested; but when the nerves, coursing as they do between the abdominal muscles and between the ribs, are injured, as in sprains of the sacro-lumbalis, psoas, and other muscles of the trunk, they may readily escape his notice; nor is this surprising when we consider the predominance of the other symptoms in all cases of severe sprain. Numbness or tingling in some part remote from the injury is of frequent occurrence, and would seem to indicate an overstretching. Such symptoms are met with when the head of a dislocated bone stretches or presses upon a nerve. Numbness usually passes off in from twenty-four to thirty-six hours, or as soon as the pressure is relieved or the stretching force relaxed. Should the symptoms 


\section{GENERAL CONSIDERATION OF SPRAINS}

persist, massage may be tried and kept up until distinct relief is afforded. When, on the other hand, the pain radiates along the course of a particular nerve or group of nerves, absolute and complete rest becomes imperative. Massage and movements do positive harm, and herein lies the inherent difficulty in treatment. We have seen that in simple sprains and strains, movement and massage are advocated while fixity is deprecated. In no class of complicated cases do the routine movements and exercises recommended by the bone-setter cause more harm than in this. The límb or joint must be given absolute rest, though not necessarily placed in a splint, until the pain has completely disappeared or is rapidly subsiding. Then very gentle friction massage may be cautiously tried. If the pain is in the least degree increased after exercise it should be forbidden. A good alternative plan to the use of massage is that of bandaging the whole limb or part of the body, as the case may be, with a bandage of stout elastic webbing. By this device it is possible to partially deplete a limb or other part of its blood. When such a bandage is retained for say five minutes or less, and then suddenly and rapidly removed, local flushing results. In this way fresh arterial blood is brought to the injured part, while on the re-application of the bandage the vitiated materials are removed by compression and hurried into the general circulation. The advantage which accrues from this practice is that the general nutrition of the nerve-endings is improved. This is especially true of the treatment of chronic cases, and in this particular class it may be necessary to remove 
and re-apply the bandage two or more times in twenty-four hours. At first, progress is slow, but after a few days, as a rule, the pain will gradually subside and disappear. The local neuritis seems to be remarkably influenced by the increased oxygenation, just as the flushing caused by taking a glass of wine will, in many persons, rapidly dispel an attack of acute supra-orbital or facial neuralgia. The repeated and alternating depletion and flushing of the injured tissues stimulates the local circulation. Whatever the true explanation may be, the fact remains that the effect is good and that it is nearly always speedily beneficial. In the more chronic forms, those resulting from neglected treatment in the first instance, electricity in the static form has been found useful, while massage may or may not succeed. Blistering, and even the point of the actual cautery, have been employed on many occasions to counteract the intensity of the pain as it courses along the nerve-trunk. Opiates should be avoided, because these cases often last a long time, and the continued use of drugs is baneful and can only at best give but temporary relief. One of the most rapid and obstinate forms of the wasting of muscles results from gross nerve lesion. It is more rapid, more complete, and more lasting than any of the forms that result from mere disuse. 


\section{Sprains with Fractures and 'Fracture- SPRAINS'}

The treatment of this class differs from that of a simple sprain in certain particulars. Thus, where small fragments are detached, pulled away with the ligaments or tendons, it becomes necessary to aim at giving sufficient rest to enable if possible bony union to take place. Elastic pressure, applied in the same way as for simple sprain, is useful, for, when employed, a modicum of rest is assured without the necessity of making use of actual splints. Passive movements, carefully made, may be begun from the very earliest, but any forcible voluntary efforts which would tend to separate the bony fragments must be strictly prohibited. Massage may be used all the time, but cautiously at first. Exercises are of value late in the case. Where there is fracture without separation, as in fissures in the lower end of the fibula or tibia, the metacarpals or small bones of the carpus, and where there is but slight effusion, strapping as for an ordinary strain is convenient. Passive movements are more helpful on the whole than active; the latter, however, may be allowed, but only to a limited extent and providing no strain is directly put upon the region of the fracture. The patient may, in the case of the smaller joints, be allowed to flex or extend the joints fully without using much force. Exercises should be encouraged after the bones have had time to unite, but not before. Unless these rules are strictly adhered to, imperfect recovery will result, for faulty or incomplete union must follow whenever forcible voluntary 


\section{GENERAL CONSIDERATION OF SPRAINS 39}

movements are permitted in the early stages. The main difference between the treatment of simple sprains and the treatment of sprains complicated by fracture is, that, in the former, voluntary or active

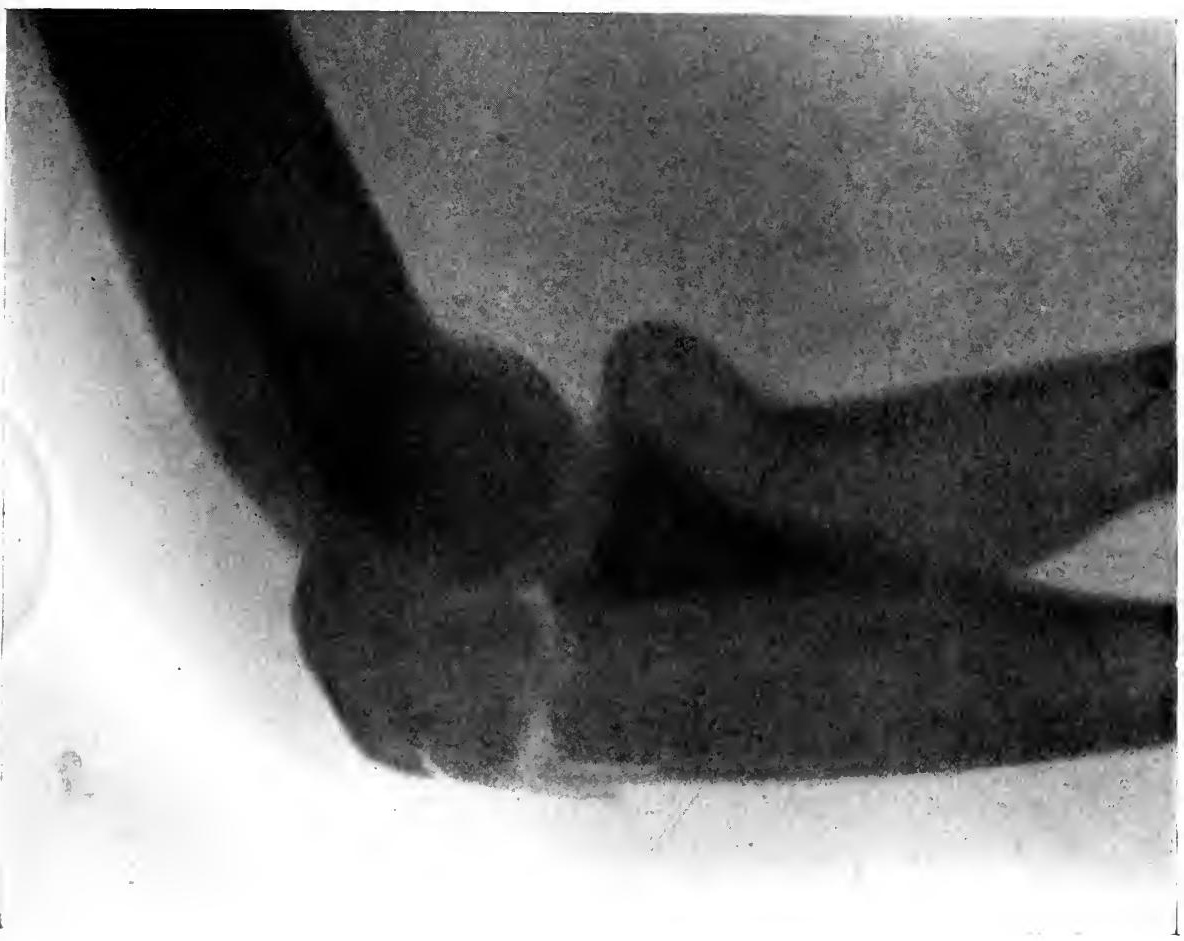

FIG. 6. Radiogram of a fracture of the olecranon without displacement, at first regarded as a sprain. To illustrate 'fracture-sprain'. Massage and early movements in such a case are absolutely contra-indicated.

movements are permitted and encouraged from the very earliest stages, and are essential to a speedy recovery; in the latter they must be secondary to passive movements and allowed only in the later stages. Massage is of equal service to both classes, 


\section{GENERAL CONSIDERATION OF SPRAINS}

and, speaking generally, may be begun early. In simple sprains passive movements are of secondary importance, while in those complicated with fracture they are of greater service than are voluntary exercises, and especially is this true of the early treatment; exercises are helpful in both classes in the later stages.

Many sprains with fracture have resulted in stiffness, from the fact that the nature of the injury was not at first recognized and treatment was applied as for simple sprain. Vigorous treatment by massage and early movement by exercises are unsuitable where they are concerned. It is of the utmost importance that in all cases of unusual severity, and in doubtful cases, an X-ray phrotograph should be taken from different aspects. This affords our best means of diagnosis, upon which alone must depend the success or otherwise of treatment; moreover, without a correct diagnosis much unnecessary suffering will be experienced and considerable time lost. 


\section{CHAPTER III \\ THE SEQUELÆ OF SPRAINS}

Many disabilities result from the faulty or careless management of sprains, while some cannot in any way be ascribed to the fault of the surgeon. They arrange themselves into two categories : the preventable and the unavoidable. Of the former group there are many, of the latter, few.

Of the preventable forms, we meet with :

(1) Stiff joints and painful joints.

(2) Atrophy and wasting of muscles acting on joints.

(3) Recurring or relapsing synovitis in joints.

(4) A relaxed condition of joint known as 'wobblyjoint' or 'weak-joint'; the opposite condition to Group 1.

(5) Persistent pain in a joint.

(6) Crippled or deformed joint.

(7) Improperly united muscles and tendons.

(8) Ankylosis.

Stiff and painful joints as a result of sprain are almost invariably due to prolonged fixation in splints or other rigid apparatus, assisted by the prohibition of or delay in beginning movements during the treatment. For some reason, not altogether independent of textbook teaching in the past, it has been the habit to regard so-called rest as a primary and all- 
pervading essential in the treatment or management of any injury. The treatment of sprains, perhaps, of all injuries has suffered most from this doctrine. That a joint, designed and constructed as it is by nature for movement, should be dealt with in precisely the same way when it suffers violence as a bone arranged and planned for a purpose the very opposite, seems strange; yet, strange as it may seem, it is only too true that even at the present day there are surgeons who treat sprained joints in precisely the same way as they do-fractures. Nothing can be more harmful to the normal movements of a joint than a prolonged and constant use of splints, and if for any reason a splint should be considered advisable, the same should be removed at least once a day, so that movements in the joints may be allowed. The writer, for over eighteen years, has never had occasion to use a splint, and he firmly believes that sprains will do better if left alone without any special treatment than when they are subjected to prolonged fixation. The bugbears 'inflammation' and the 'dread of tuberculosis', would seem to haunt the minds of some as if they were veritable demons. One need hardly point out that in the absence of local temperature such fears are ungrounded.

Joints remain painful and stiff as the result of immobilization, whereby the surrounding tissues become matted together; the nerve-endings are pressed upon by exudation products which are unabsorbed, and are injured by an impeded and sluggish blood-supply deficient in oxygen ; circumstances which all lead up to an impaired nutrition. 
Atrophy and wasting of muscles acting on joints. This results from two main causes: $(a)$ a definite injury to the nerve-supply, and $(b)$ disuse, often associated with the long-continued application of splints and other apparatus during the course of treatment.

(a) Examples of the former class are the changes observable in the distal portion of a ruptured tendoachillis which has remained ununited. The distal part of the tendon, cut off from the nerve-supply to the muscle fibres on the proximal side, atrophies, shrivels, and takes on the consistence and appearance of a piece of wash-leather. Again, when the adductor longus muscle becomes torn from its pelvic attachment, in a case of so-called 'rider's sprain', it is no uncommon circumstance to find the muscle almost completely wasted away. The rapid wasting in the deltoid, resulting from overstrain or partial rupture, is doubtless due to nervous influences-possibly the cutting off of the nerve-supply.

(b) Examples of wasting from disuse, arising as a consequence of unduly prolonged fixation and tardy resort to movements and exercises in the treatment of a large joint like the knee or shoulder, are of too frequent occurrence ; and it cannot be emphasized too often, in connexion with this, even at the risk of being tedious, that the wasting of muscles begins almost immediately after an injury, and that passive movements by themselves have little or no beneficial or preventive influence. Voluntary movements, however slight in the beginning, gradually increased as they are better tolerated, assisted by the skilful application of massage, will alone retard or prevent this form of atrophy. Later on, properly regulated 
exercises, planned so as to work against resistance, do much to promote complete restoration to usefulness. Voluntary movements and massage should be persevered with in both forms of wasting, but the surgeon must be prepared for disappointment in a

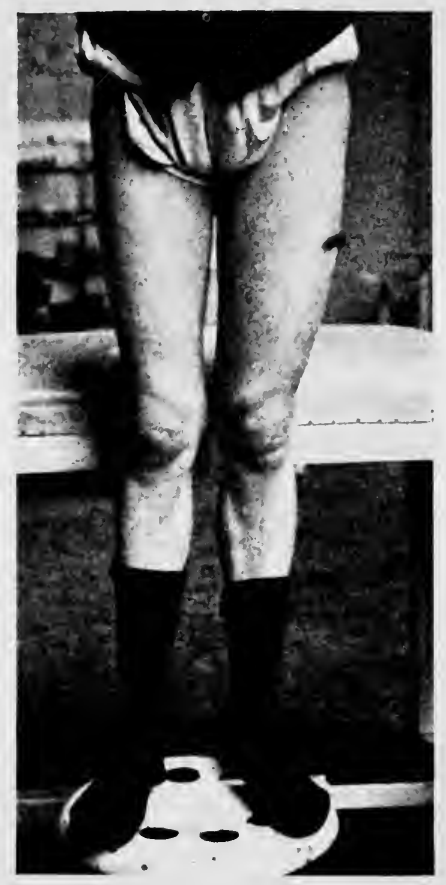

FIG. 7. A case of atrophy from disuse. Right limb one inch less in circumference than left.

certain number of cases, notably those in which a gross nerve lesion is the primary cause.

Fig. 7 is the photograph of the limbs of a young gentleman who in falling from a bicycle twisted his right knee. The right limb affords an excellent example of atrophy from disuse. The picture was 
made eighteen months after the sprain, and at that time the limb measured exactly one inch less than its neighbour. At no time had a splint been applied, and as no nerve lesion had been found it must be assumed that the shrinking was caused by disuse alone. The knee was encased in bandages for weeks at a time, and the patient was only allowed, for the greater part of the time, to walk with crutches, for ' whenever he put his foot to the ground the joint swelled up and became painful'. The early part of the treatment was carried out in France, the late in London. On no occasion was he seen or treated by an unprofessional person, but from first to last was under the care of several of our greatest surgical authorities. During the eighteen months in which he was lame he was treated in various ways and by many methods. Beginning with bandaging and rest, massage (manual and electrical), points de feu, blisters, Scott's dressing, radiant heat, Bier's hyperæmic and congestive treatments, kataphoresis, were in turn tried. No expense was spared, nothing neglected. The patient thought that kataphoresis with iodine checked the tendency to relapse of the synovitis, while the active muscular exercises which he was eventually taught to practise strengthened the limb. When last seen, the limb was still smaller than the other, but he was able to walk eight or ten miles and to bicycle over sixty miles without fatigue. The case is instructive, as it tends to prove that no form of surgical treatment will prevent atrophy if the muscles themselves are prevented from taking part in active movements and exercise.

Recurring or relapsing synovitis. In a certain 
number of severe articular sprains, in which there has been considerable tearing of the external ligaments, the capsule and synovial membrane, the effusion, which at first was copious, clears up readily and satisfactorily under rational treatment, but as soon as the patient begins to walk or move the joint it again refills. The tension may be high or moderate, and there may be some local increase in temperature. When elastic pressure is applied the effusion is absorbed in a very short time, but relapse after relapse, recurrence after recurrence, follow, and lead to a general relaxation of all the ligaments. This troublesome condition is generally due to the presence of an adhesion or adhesions within the joint. The simple folding of a small and insignificant portion of the synovial membrane upon itself, or some irregular attachment by a tag of the capsular ligament which has become curled inward towards the articular surfaces of the bone, are sufficient to produce synovitis whenever dragged upon during the ordinary movements of the joint. Such a state of affairs is best prevented by the routine advocated in a former chapter-that of putting every severely sprained joint through its normal movements before any treatment whatever is commenced, an anæsthetic being used if necessary. When adhesions have formed, they are best remedied by forcible movements under an anæsthetic, the movements being followed by the usual course adopted for the treatment of recent sprains. If the effusion should keep on recurring in spite of this, it is well to perform an exploratory arthrotomy to remove any band or adhesion existing within the joint. The knee is the 


\section{SEQUEL $Æ$ OF SPRAINS}

joint which is most prone to these recurrent attacks. In some of the most obstinate cases the condition is due to the presence of thickened synovial fringes. The accompanying photograph shows the usual

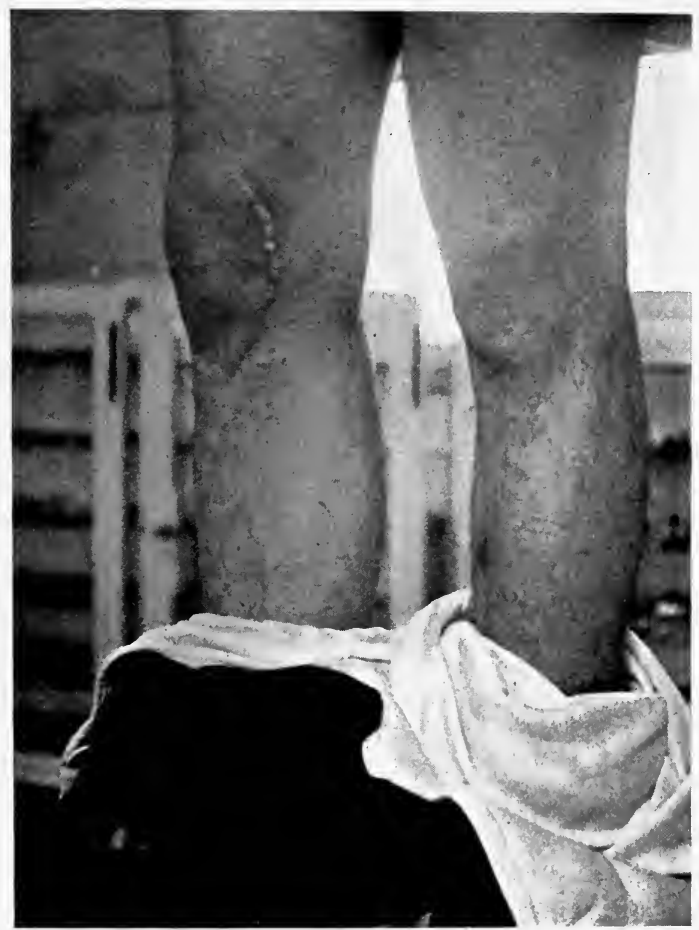

Fig. 8. To illustrate the scar left from the operations for exploratory arthrotomy and removal of internal semilunar cartilage. From a case three months after an operation for removal of synovial fringes.

incision employed by the writer whenever he makes a digital exploration of the knee-joint. It extends in a slightly curved direction over the inner side of the joint for about four inches, having its centre opposite the internal semilunar cartilage. When the skin and 
fasciæ have been divided, the capsule comes well into view. This, in its turn, is divided parallel with the skin incision; the joint is then opened. By everting the outer edge of the wound, the under surface of the patella, with the infra-patella pad of tissue (ligamenta mucosa et alaria) may be freely exposed to view. The finger of a gloved hand may now be inserted and a careful exploration made of every corner of the articulation. The synovial membrane, in these cases, is often of a pale yellowish fine-dotted appearance, slightly granular on its visceral surface. The secretion varies in different individuals and at different stages, according as to whether the effusion is recent or late. While at times it is liquid and slightly viscid, at others it may be of the consistence and appearance of boiled sago. If the synovial membrane is examined microscopically it presents the usual characteristics of a catarrhal inflammation. Adhesions, however small, should be clipped away with scissors, and any hypertrophied portions of synovial membrane carefully removed. It is imperative that all bleeding points should be carefully attended to ; dry swabbing is much preferable to douching or irrigation. The writer has almost entirely abandoned the practice of douching out joints, and sponges with dry swabbing instead. A gauze drain is inserted at the middle of the capsular wound, and the rest of the capsule is brought together with catgut sutures; the skin wound, with silkworm gut. A bicyanide gauze dressing, covered by a thick pad of salicylic or boracic wool is then applied, but no splint. The drain should be removed in twenty-four hours, the wound then sealed and left 


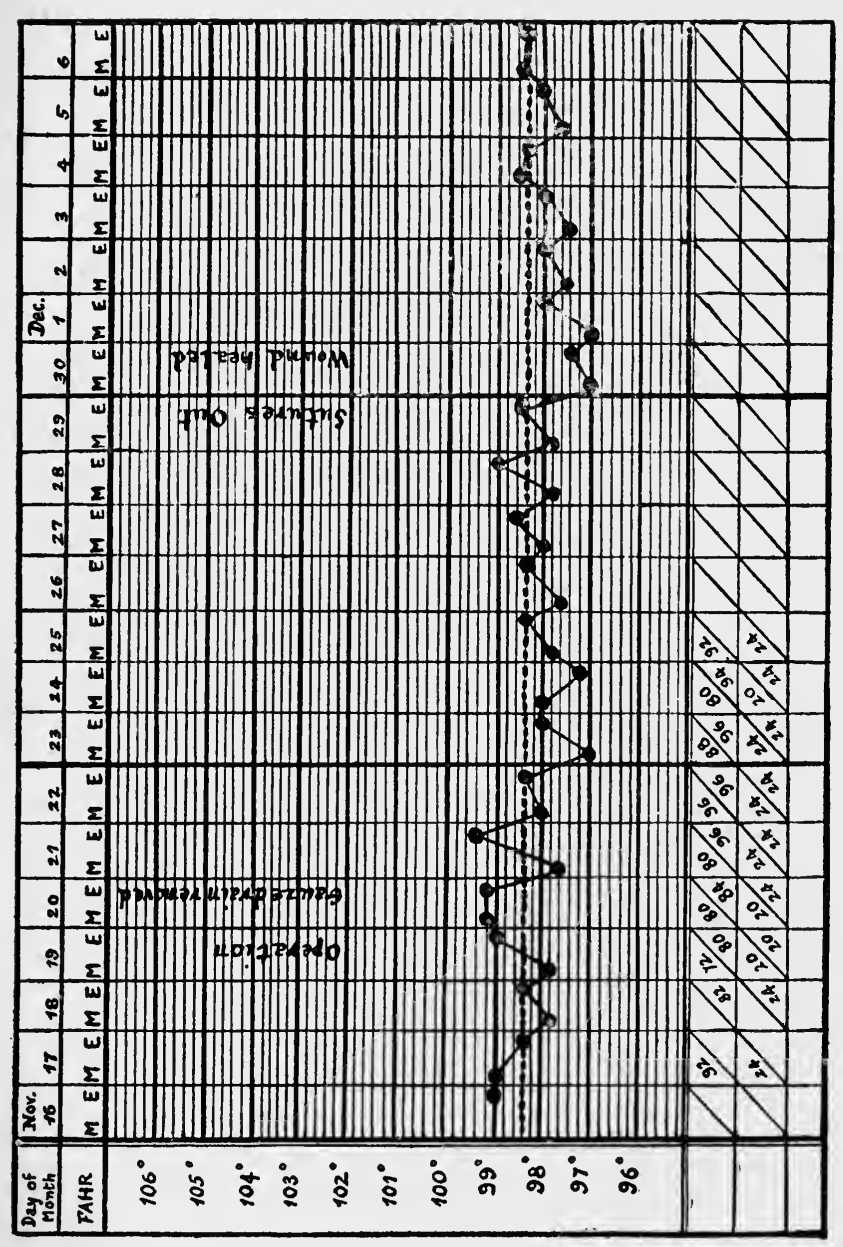

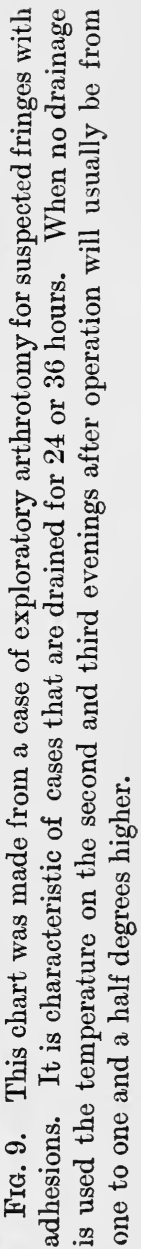


undressed for nine days, providing the temperature remains satisfactory. The accompanying temperature chart is characteristic of exploratory arthrotomy. There is usually a rise of temperature for the first two days; this should subside on the third or at latest on the fourth day. Gentle passive movement may be tried at the first dressing, twenty-four hours after the operation; massage and voluntary movements are best postponed until after the removal of the stitches, usually from the eighth to the ninth day, and they must be both continued until the joint becomes firm and strong and the tendency to muscular atrophy and to recurrent effusion has passed.

A generally relaxed condition in a joint, popularly known as 'wobbly-joint' or 'weak-joint'. The socalled 'wobbly-joint' is the result of muscular atrophy in the proximal muscles of a sprained joint. The accompanying photograph (Fig. 10), taken from a case in which fixation in plaster of paris for the greater part of six months was permitted, on the assumption that the slight elevation of local temperature indicated early tuberculosis, is typical and characteristic of the condition.

The subject was a young military officer who, while playing at football, twisted his left knee. The pain was confined to the outer and posterior regions of the joint. At the time, no locking or impaired mobility was experienced, and he was immediately able to walk. Some swelling of the joint was noticed next day when a bandage which had been applied was removed. He remained on the sofa, but being in India and many miles away from any medical attendant he began to walk about in a few days. At the end of ten days, the 'pain and swelling having quite subsided', 


\section{SEQUELA OF SPRAINS}

he again played football. Pain quickly returned, and this time was referred to the centre of the back of the knee. $\mathrm{He}$ experienced no sensation of anything giving or tearing,

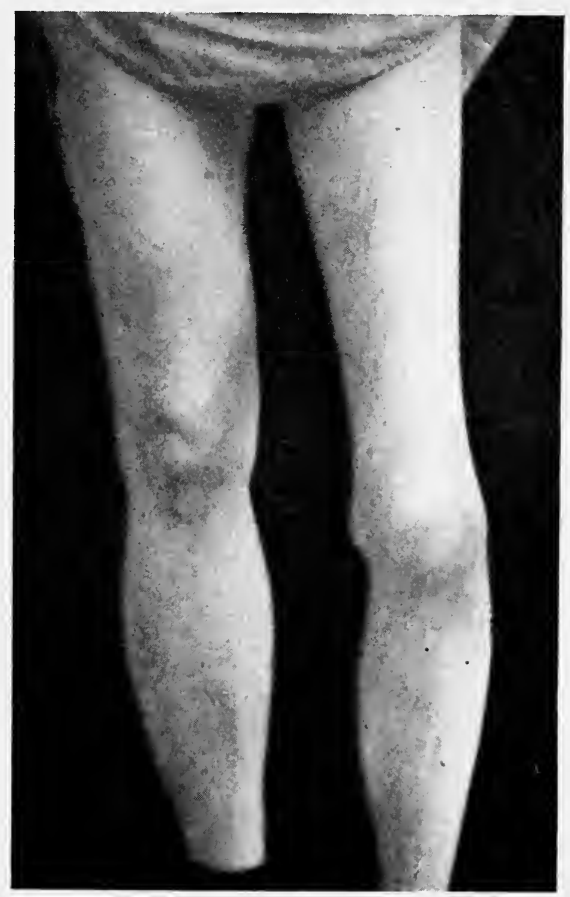

Fig. 10. Рнотоgraph оғ вотн тнighs. The left knee shows deformity from unreduced semilunar cartilage (internal). The limb had been retained for nearly six months in plaster splints. Note marked atrophy of the muscles of the thigh on proximal side of the joint, also semiflexure of the knee, with hypertrophy of the muscles of calf. This is somewhat charactrristic of knees long retained in splints without movement. Such a joint readily fills when exercised, and illustrates the so-called ' weak' or ' wobblyjoint'.

and although he could bend and straighten the joint freely, it rapidly swelled up, as he described it, ' to an enormous size.' Surgical advice was now sought, but not obtained until thirty-six hours later. Rest in bed, evaporating lotions, 
and the usual textbook routine were gone through; the swelling gradually subsided, and splints were used from the first. Three weeks later he was allowed to walk, with the limb encased in a moulded splint, but the joint at that time was weak, stiff, and painful. As he did not seem to prosper, and the joint swelled whenever he stood erect, and was hotter than its neighbour, his medical attendant thought that tuberculosis was probably beginning. For the next five months the limb was kept fixed in a plaster casement.

These details have been supplied here as illustrative of the line of treatment so often adopted, and with such untoward results. It is important to notice the more marked atrophy of the proximal muscles of the thigh as compared with those in the leg or distal to the joint. In these relaxed states of the ligaments the recurrent synovitis in most cases is a passive one ; the joint filling simply from loss of support to its capsule, secondarily to wasting and enfeeblement in the muscles which control its movement.

It is of the utmost importance that it should be clearly understood that the swelling is secondary to, and dependent on, the wasting of the muscles, and that treatment should be directed towards rectifying the enfeebled condition of the controlling muscles rather than to the joint itself. Local treatment, such as blistering and other forms of counter-irritation, are helpful only to a limited degree and will not cure the condition. Massage and movements, both active and passive, and those against resistance, must be strenuously persisted in until such time as the tone of the capsular ligament is restored or at any rate materially improved. 
The improvement will be in proportion to the amount of perseverance shown in carrying out the exercises, and the degree of recovery to the length of time that has been saved or lost before rational treatment was begun. It may take months before the strength of a limb is restored, and in some, fortunately a small percentage of cases, complete recovery must not be expected. Especially is this true when, in addition to the mismanagement by prolonged fixation, there has been extensive laceration of the tendons and aponeuroses in and around the joint.

Persistent pain in a joint is characteristic of gross injury to nerves. It is generally referred along the line of a particular nerve or group of nerves, notably after injury to the largest joints, the hip, knee, shoulder, and elbow. In some cases it appears to be of the nature of neuritis, occasioned by the bruising of the nerves themselves, or to adhesions of their sheaths to the surrounding parts analogous to the pain experienced when a nerve is caught in callus at or near the seat of a fracture. The normal and healthy nutrition of the nerve becomes altered. In a certain proportion, the pain or neuralgia is caused or intensified by gout or rheumatism; as a rule such cases are readily improved by the appropriate remedies for these constitutional diseases. The pain is generally worse at night, and in a few exceptional cases has been so severe as to necessitate the exhibition of opiates. Friction massage at the hands of an experienced masseur will generally serve to diminish its intensity, so that strong drugs are rarely needed. Aspirin in full doses will in many 
cases relieve the attacks of aching pain, and in some remove them altogether.

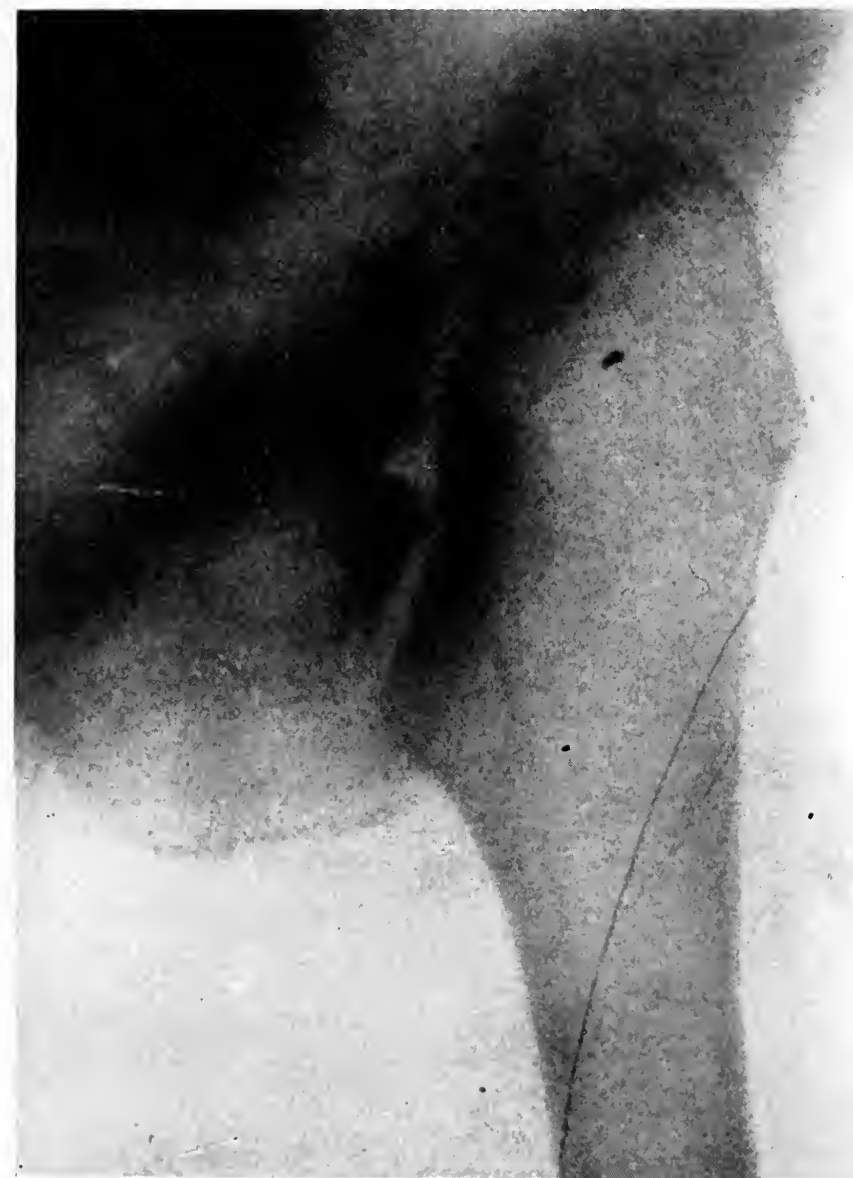

Fig. 11. False joint at right hip, the result of treatment for supposed sprain by bone-setter. The neck of the bone has been worn away by continued movements and exercises, and the patient permanently lamed and left with a deformed hip.

The crippled or deformed joint. Deformities formerly were considered to be the result of osteo- 


\section{SEQUEL E OF SPRAINS}

arthritic changes, but are now known, through the experience gained by radiography, to be frequently owing to the separation of the epiphyses or even to fractures. The accompanying radiograms (Figs. 11,

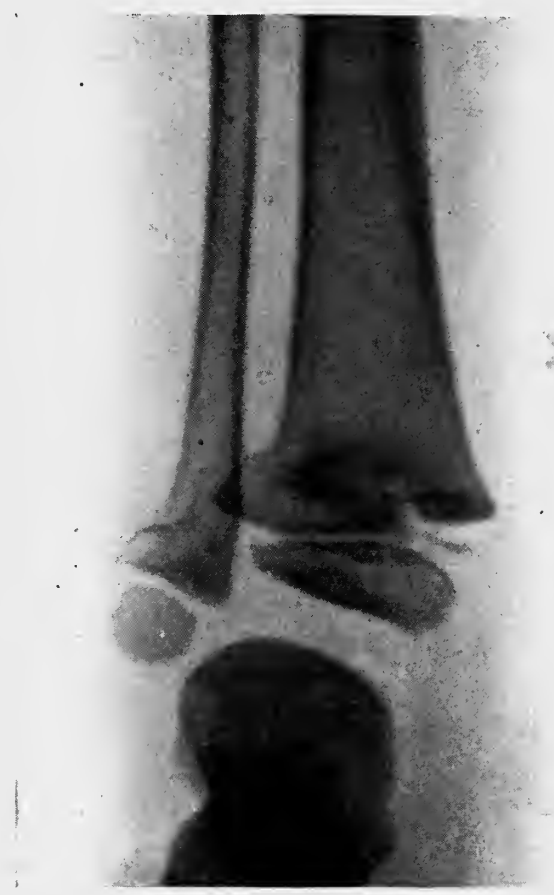

FIG. 12 is a radiogram of a deformed ankle in boy, the result of fracturesprain with separation of epiphysis of tibia and greenstick fracture of fibula -at first thought to be a sprain.

$12,13,14,15$ and 16) bear out this fact. Deformities may be met with in the large as well as the small joints ; the flattening out of the hip with atrophy of the gluteal muscles, so often represented in the older textbooks as resulting from a fall with bruising, is typical. At one time this condition was ascribed to 
some subtle change in the neck of the femur, whereas we now know that it results from a fracture through the neck of the bone. The genu-valgum or genuvarum which follows upon a sprained knee in a growing youth is frequently the result of fracture through a condyle of the femur, or to partial separa-

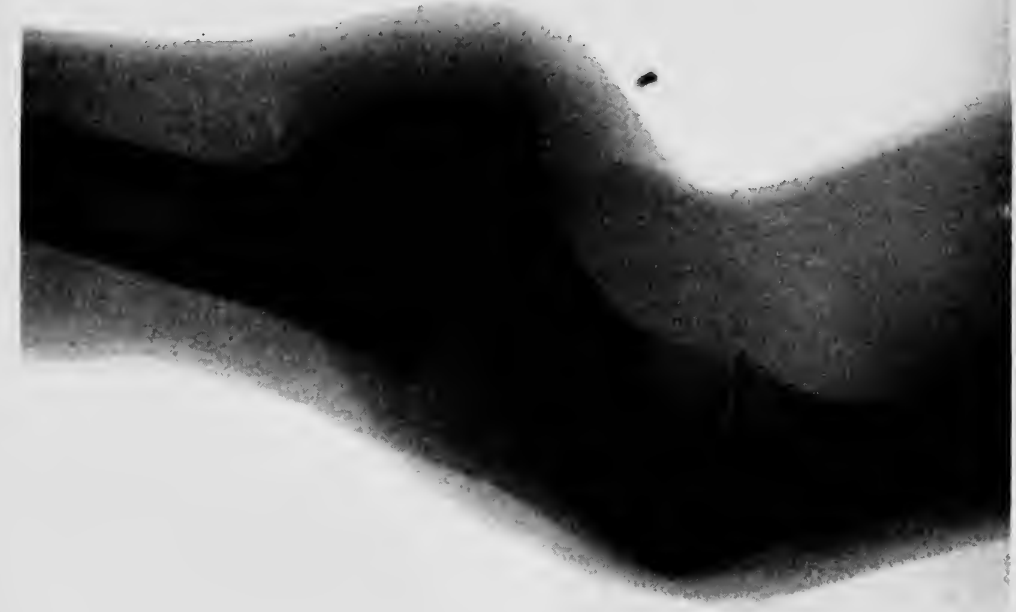

Fıg. 13. New false joint in lower third of humerus, the result of moving the limb to prevent stiffness at the elbow. Note ball-and-socket arrangement of false joint and development of a styloid-like process which had become detached by a second accident a year later. To illustrate the dangers of indiscriminate movements in the treatment of joints.

tion of or severe bruising over the growing epiphysis, either at the lower end of the femur, or upper extremity of the tibia. Examples are more frequently met with at the elbow; here, as the result of some slight strain in early life, a permanent deflection of the forearm outward with an apparent lengthening 
of the inner condyle may be seen in the adult. At the wrist injuries to the epiphysis often lead to deformity. Figs. 14 and 15 illustrate such a case.

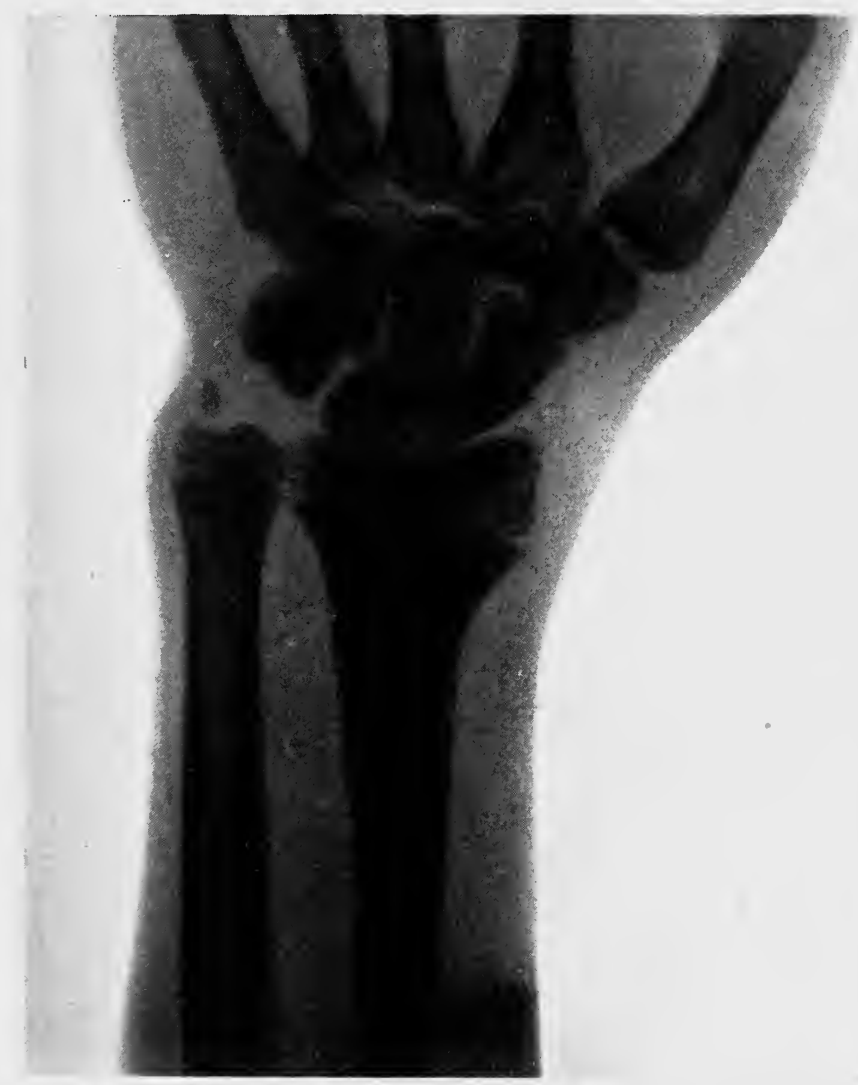

Fig. 14. Injury of wrist treated as sprain. Injury to epiphysis of radius resulting in defective growth (Mr. J. Keogh Murphy's case).

A boy of 16 two years ago fell backwards and saved himself by landing on his hand, and was treated as for sprained wrist. Since that time the wrist has gradually altered in shape. To remedy this the 
epiphyseal line of the ulna was excised and the hand put up in a slightly adducted position (Mr. J. Keogh Murphy's case). In the fingers, fractures of the phalanges are by no means uncommon; wicket-

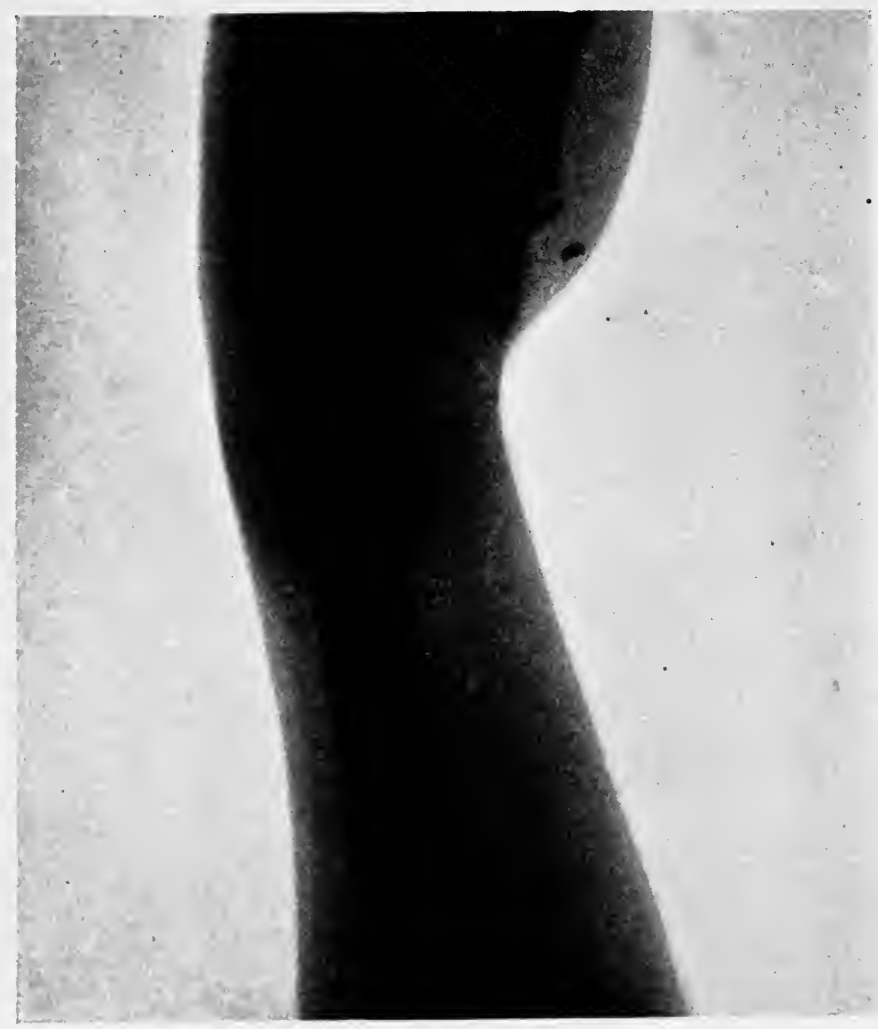

Fig. 15. Same case as Fig. 14 seen in profile.

keepers are specially liable. Without the X-rays it is next to impossible in most cases to make a correct diagnosis; a wise surgeon will therefore do well to bring them to his aid whenever it is practicable. 
Improperly united muscles and tendons. As a consequence of laceration of important muscles like those of the thigh, the adductors, as well as the extensors and flexors of the knee, wide separation is

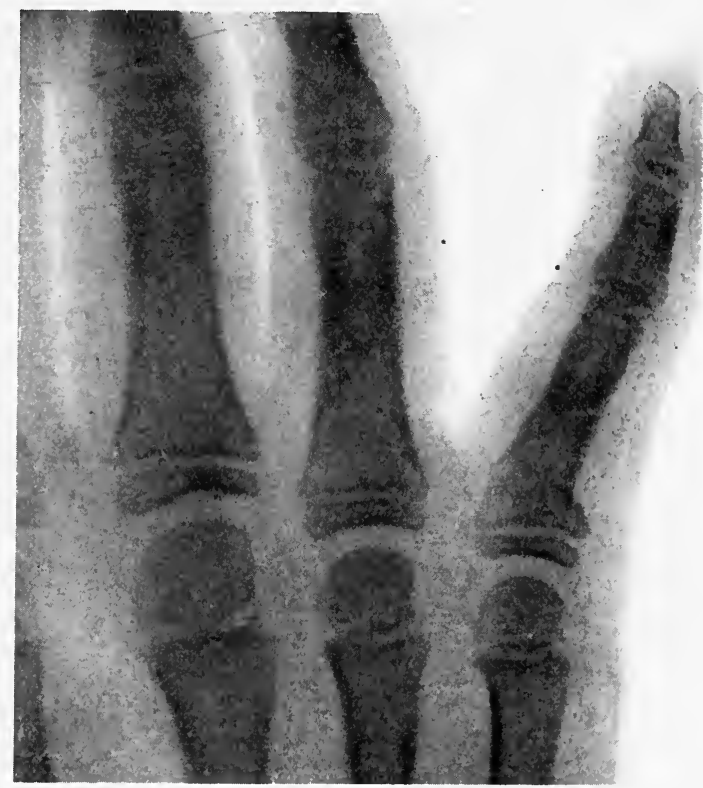

Fig. 16. Radiogram of fracture of the first phalanx of little finger of a child, treated previously as sprain. Note the absence of displacement of the fragments. The joints, both above and below, were full of fluid and masked the symptoms of fracture. An injury at the junction of the upper epiphysis with the shaft of the bone led to permanent deformity.

apt to take place between the torn ends. Unless in the course of treatment the fibres are brought together, so that more or less firm fibrous union takes place, the scar is apt to stretch at a later period and give rise to insecurity and disfigurement. The proximal portion has always a tendency to be drawn away from the seat of injury. It is fortunate 
that the other muscles of the same group will as a rule develop and increase in size proportionately to make up somewhat for the deficiency and actual loss of power in the torn muscle. It has been found, however, that rupture of the rectus femoris and portions of the deltoid muscle have seriously influenced the stability of the knee- and shoulder-joint respectively. It is important, therefore, that close and complete apposition should be secured from the first, even if it is necessary to obtain the union by means of an open operation. No undue strain should be permitted until the united parts have had full time to become firm.

Ankylosis may result from severe sprains. Fortunately, the condition is an exceedingly rare one, if we except those cases of stiff joint which at first sight similate true bony ankylosis but which readily yield to 'breaking-down' under an anæsthetic. Such a case usually originates from a sprain with fracture, the true nature of which was unrecognized and treated by excessive and ill-advised movements. The enforced movement gives rise to increased formation of callus in the neighbourhood of the articular surfaces, which not only tends to mechanical locking, but affords opportunity for new and extensive adhesions to form.

\section{Unavoidable Sequelæ}

These permanent and remote consequences of sprain are entirely beyond the control of the surgeon, but are nevertheless of much interest to him in so far as they influence, in the main, his prognosis. They consist of :- 


\section{SEQUEL E OF SPRAINS}

1. Local paresis or paralysis.

2. Ossifying myositis, as concerns sprains in muscles, and

3. Osteo-arthritis in relation to articular strain.

4. Loose bodies in the joints.

FIG. 17. Ossifying myositis in muscles of thigh, the result of a blow. Note the crescent-shaped mass attached to the middle of the shaft of the femur anteriorly. From an X-ray photograph taken nine weeks after the accident.

1. Local paresis or paralysis result for the most part from overstretching or laceration of nerves or their branches; but it is rarely met with. When the torn ends of a muscle are widely separated, as the consequence of sudden violence, the nerve of supply may be damaged. Repair, in the generality of instances, results from treatment, and cases of permanent defect are rare; when they do occur they 
are unavoidable. Friction and kneading massage may do good and will need to be carried on for a considerable time. Electrical stimulation, in certain cases, may also assist.

2. Ossifying myositis is also rare. It is almost invariably met with in the large muscles and in those

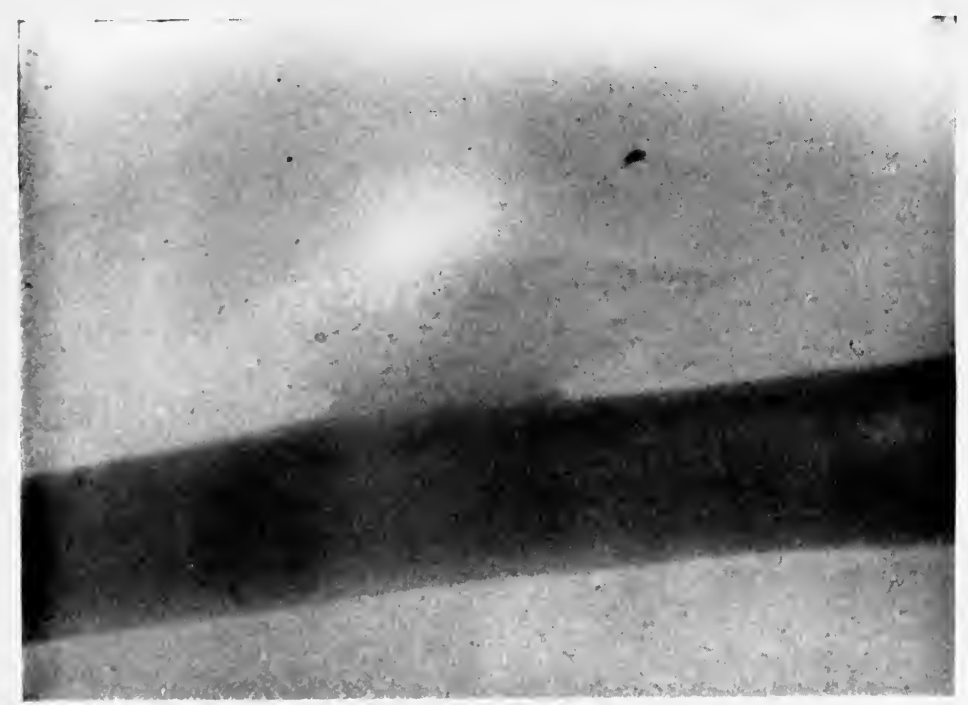

Fig. 18. Myositis ossificans in extensor muscles of the thigh, result of a kick by a horse. Note the intimate connexion of the mass with the anterior surface of the femur. From an X-ray photograph taken sixteen weeks after the accident.

whose fibres take origin directly from some rough bony ridge, or which are directly attached to the periosteum without the intervention of much tendinous material. The writer believes that in all such cases portions of the periosteum become detached with the muscle at the time of injury, and are pulled away from the bone by the contraction of the muscular fibres. The detached periosteum soon 
SEQUEL E OF SPRAINS

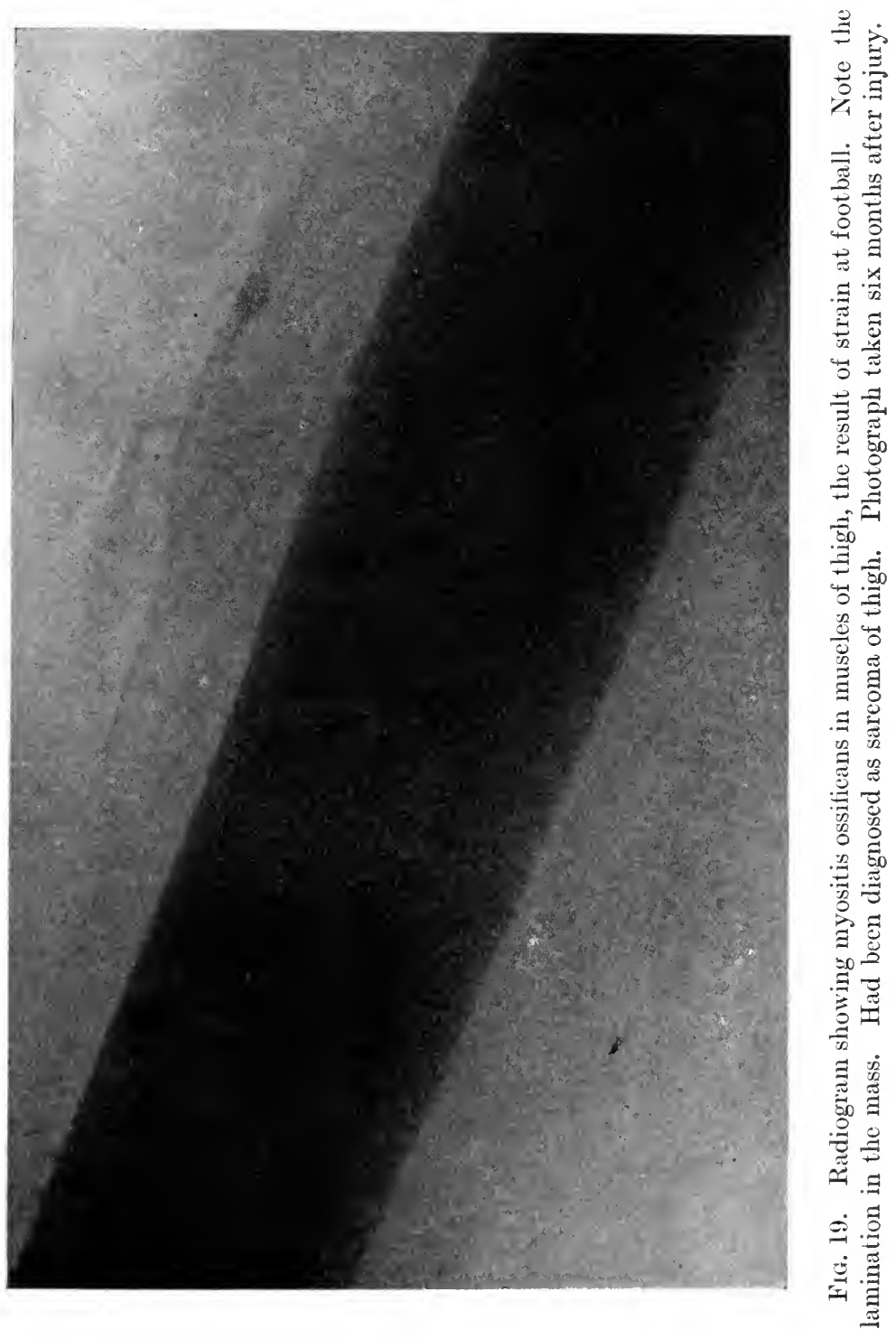


becomes enveloped in the subsequent extravasation, which for the most part consists of blood-clot, for the vessels of the periosteum bleed profusely whenever they are torn. The osteo-blasts, which are normally numerous between the deeper layer of the periosteum and the bone, stimulated in their new situation partly by the increased blood around them, partly by the contractions of the muscle, and perhaps by movements of massage during the treatment, proliferate and proceed to the deposition of new bone. This theory is based upon the following observation. The accompanying X-ray photographs were taken from two different cases; in the one (Fig. 18) the bony growth is seen projecting from the anterior surface of the femur into the quadratus extensor muscle. This resulted from a direct blow, the kick of a horse, in an adult man. It will be noticed, in comparing it with the next picture (Fig. 19), that an ossification has resulted in the same muscle and at about the same situation in the thigh. The second picture represents, however, the result of a strain of the muscle produced while the patient, a youth, was playing at football. In this case, what happened was probably that the periosteum was stripped from the shaft of the bone, owing to a sudden and violent contraction of the crureus muscle, and is therefore an example of indirect violence. The new growth of bone in this case lies in the belly of the muscle, parallel with, but separated from, the femur; it is seen to be laminated. The pathological condition in this particular instance is similar to that pertaining to callus-formation at the seat of a fracture. The periosteum being torn, the osteo- 


\section{SEQUELA OF SPRAINS}

blasts are set free into the blood-clot which surrounds the fragments of bone, and stimulated by the movements of the broken fragments, rapidly proliferate, and produce new bone.

On one occasion the writer was able to verify the existence of torn periosteum with its deposition of

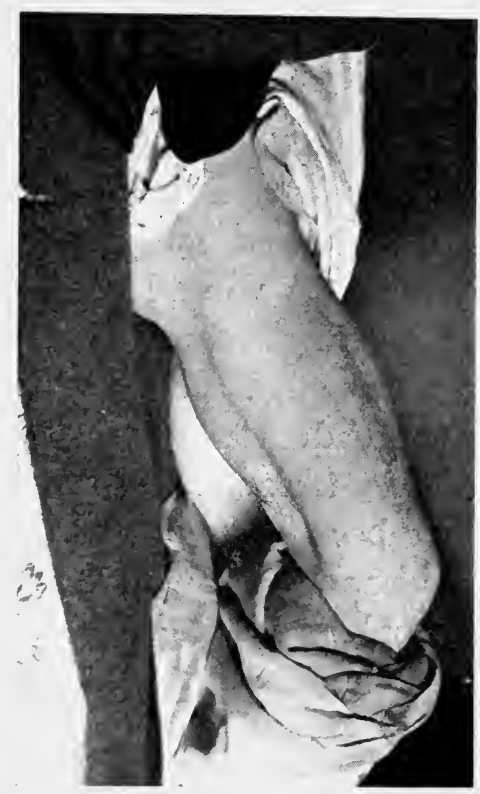

Fig. 20. Photograph of thigh showing a swelling anteriorly about the middle, due to ossifying myositis in the extensor group. The result of a football strain.

new bone, in a case of extensive hæmatoma in the calf. The patient, a gentleman aged twenty-two, was thrown from his horse while hunting, and being caught in the stirrup was dragged. In addition to other injuries he tore away portions of his soleus muscle from its origin at the oblique line of the tibia. When, ten days after the injury, an incision was WHITELOCKE 
made into the enormous swelling that resulted, for the relief of the much-impeded circulation in the foot, and after the clots had been removed, right along the whole length of the muscular detachment were to be seen and felt signs of commencing ossification. The detached portions of the periosteum still adhered to the muscle fibres and were rough and gritty to the touch. Ossification had already begun in the clot which lay between the soleus and the gastrocnemius muscles.

The detached portions of the periosteum were at the time carefully clipped away with scissors, the clots removed, and the wound closed. It spite of these radical measures, a sharp spicule, owing probably to some detached portion having escaped notice at the time, developed and grew from the oblique line of the tibia. Doubtless, if the other portions had not been removed at the time of the operation, considerable ossifying myositis of the soleus, and probably of the gastrocnemius also, would have resulted. Other examples have been met with in the adductor longus and gastrocnemius at their origin, and in the deltoid at its insertion. In 'rider's sprain' the adductor longus, as well as the pectineus, are usually separated at their pelvic attachments, close to the bone, and in the tearing of these muscles it is probable that the periosteum is separated from the pelvis and that the new bone is deposited among the muscle fibres which arise from it. Ossification in the deltoid and gastrocnemius commences close to their attachments to bony ridges, the one from its insertion, the other from its origin. Cases have been reported where the 
condition was described as a 'calcareous deposition' in the muscles, but, in the cases above described, true bone was developed histologically identical with the structure of the cortex of a long bone.

The ossification of a muscle may be complete, as in the adductor longus, but is generally only partial. The symptoms of ossification develop slowly; at first there is usually little pain, and all that the patient complains of is that he has a swelling which aches, and that the limb is heavy and the movements in the affected muscles slow and increasingly difficult. The joint controlled by the muscle becomes limited in its movements, and this disability increases as time goes on. The muscles increase in size, are but slightly tender, and become gradually harder. In the later stages and in the worst cases they may become completely rigid, but this is fortunately unusual.

In a few cases, a certain but limited degree of improvement is noticed, owing partly to a shrinking of the new bone (as shown in Figs. 17, 18, radiograms taken at different stages of the same case) and partly to stretching of the muscles as they are more and more exercised.

An accurate diagnosis is of the greatest importance. Amputation through the thigh has before now been performed under the impression that the surgeon was dealing with a sarcoma growing from the periosteum of the femur. In one of the cases described before, the early diagnosis was that of tumour, but radiography saved the situation. All doubtful cases should be submitted to the X-rays.

Preventive measures are of very great importance, for other means, short of complete removal of the 
muscle with the hope that the neighbouring muscles may develop and take on some of the duties, are of no avail. Where large hæmatomata arise, either as the result of direct violence or the consequence of strain, in situations where these ossifications are known to occur, it is a wise plan to operate early. By making a free incision into the muscle through its sheath, and by turning out all clots and removing all torn fibres of periosteum, much may be done to prevent the occurrence.

When the process has actually commenced, rest is essential ; nothing that will encourage new growth, such as friction massage, should be permitted. Movements, passive as well as active, for the same reason must cease until such time as all tendency to increase has gone by. Later, massage may assist in making the remainder of the muscle more supple, but the main portion of the muscle will for the most part remain permanently useless and the condition unalterable. Entire removal of the growth may be considered when the muscle is completely rigid and useless, especially if it is a source of inconvenience. A rigid adductor has been excised to enable the individual to ride.

3. Osteo-arthritis. The connexion of these changes with injury is undoubted, but what the exact causal relationship is, remains still a matter of conjecture. Osteo-arthritis is more prone to arise in persons of middle and advanced life than in the young and growing; it is possible that there are many varieties of the degeneration influenced, or led up to, by different predisposing causes; some persons would seem to be more liable than others. 
The whole subject of the etiology and pathology of osteo-arthritis is so little understood at the present time that a prolonged discussion at this

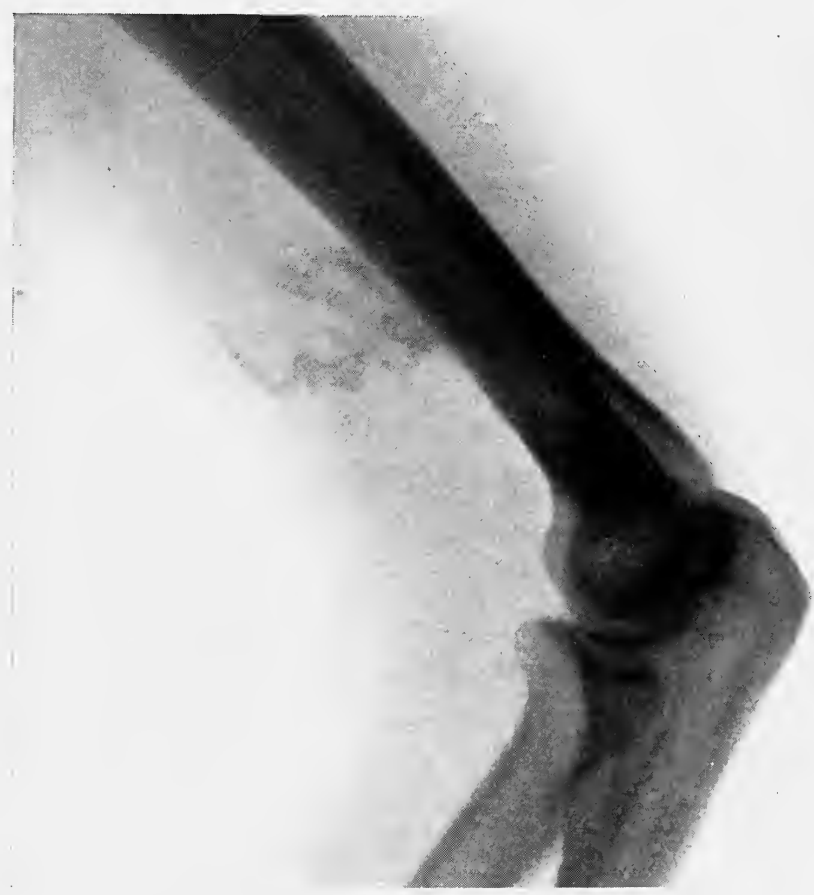

FIG. 21 is a radiogram of an arm showing the condition of myositis ossificans in the brashialis anticus muscle. The injury was caused by the arm having been caught in a chain and violently pulled. The periosteum had evidently been damaged at the time. The new bons developed in the brashialis anticus, but did not involve the biceps in front of it. The picture was made some weeks after the accident.

place would serve little purpose ; that it follows not infrequently upon injury, even upon slight injury, is an indisputable fact, and so also that it usually results in those cases in which recurrent effusion of a chronic or relapsing type have been manifest : in 
fact, just in that particular group of cases that is usually treated with enforced immobility for long periods. No satisfactory means either of preventing or remedying the condition, when it has once been established, are known to the writer, nor is it possible to prognosticate with any certainty that this or that particular injury will or will not be followed by these slowly-forming, yet subtle and progressive changes, so frequently found in and around the bony surfaces of joints.

4. Loose bodies in joints may be grouped with the unavoidable sequelæ of sprains, and while in many instances, more especially in certain varieties, a traumatic origin may be easily traced, in some the relationship, though strongly suspected, can hardly be said to have been satisfactorily established. There is still much controversy as to the precise etiology and pathology of loose bodies.

These structures are not infrequently met with, and may be arranged for convenience under two primary headings :

1. Those introduced by accident into joints from outside, and include such foreign bodies as needles, bullets, and pieces of glass.

2. Those originating in the joint itself. It is with this second group that we are for the present concerned.

Loose bodies originating in the joints. Under this grouping are two further subdivisions.

(a) Loose bodies which result from the detachment of structures which normally form part of the articulation ; as examples we find detached portions of semilunar cartilages and pieces of articular car- 
tilage pulled from the subjacent bone by various forms of violence.

(b) Those derived from the growth or formation of structures which do not form part of the joint in normal circumstances.

The commoner type of loose body belongs to this second subdivision, and is developed within the joint.

Three well-known conditions are met with.

1. The so-called 'rice bodies'.

2. Detached hypertrophied villous processes of the synovial membrane.

3. True loose cartilages which, however, by some are said to be due to the development of true hyaline cartilage in the synovial fringes and not to any traumatic influence.

Loose bodies derived from detachment of portions of inter-articular cartilages, and of pieces of articular cartilage or bone within joints will be treated of in a succeeding chapter, when the subject of 'internal derangements of the knee' come under consideration.

That they are frequently the result of a severe sprain few will deny.

Fibrinous loose bodies (corpora oryzoidea) may be mentioned, and though their connexion with sprain is a remote one it cannot be denied that on some rare occasions they do unmistakably result from sprain.

Since these are generally associated with tuberculous changes in synovial membranes, or with such affections as Charcot's disease or arthritis deformans, their consideration need not be enter- 
tained in the present treatise. They would seem to be sufficiently treated of in the textbooks on general surgery.

Bodies composed of organized connective tissues, such as fat, fibrous tissue, cartilage and bone,

FiG. 22. Radiogram of a knee-joint, showing a partial detachment of a small portion of the rim of the external condyle of the femur. The fragment was loosely attached to the periosteum, but being partially movable produced chronic synovitis.

may be found growing from synovial membranes in joints which to all intents and purposes appear to be healthy, as well as in those associated with some form of general disease.

As to the origin of this type of loose body authori- 


\section{SEQUEL $Æ$ OF SPRAINS}

ties differ. While most surgeons regard them as overgrowths of the synovial fringes within the joints, some maintain that they invariably arise as a result of injury by detachment of a portion or portions of an articular surface.

König regards them as due to a detachment of portions of the articular surfaces by a morbid process to which he has given the name of osteochondritis dissecans.

On the other hand there is much to justify the view that they may come into existence in the same way as innocent new growths do in other parts of the body, and are therefore really neoplasmic in nature.

The accompanying radiogram (Fig. 22) was made from an injured knee-joint. It shows the partial separation of a portion of the external condyle of the femur.

It is probable that in the course of time this fragment would have become wholly detached and given rise to a loose body freely movable in the articulation.

Fig. 58 is from a photograph taken of a portion of a patella which had become detached some years previous to its removal. It should be noted that this specimen presents for the most part a smooth and polished surface. Even that portion of it which originally at the time of its first detachment was rough and irregular is seen to have become smoothed over by the growth over it of hyaline cartilage. The cartilage would seem to have grown over the specimen from its circumferential margin so as to eventually encroach upon and completely cover over its frac- 
tured surface. The result is that from what was once an irregular and somewhat flattened fragment, we find a smooth and polished, somewhat rounded, loose body.

Fig. 23 represents a photograph taken of structures removed from the knee-joint of a man of middle age. The patient had detached a portion

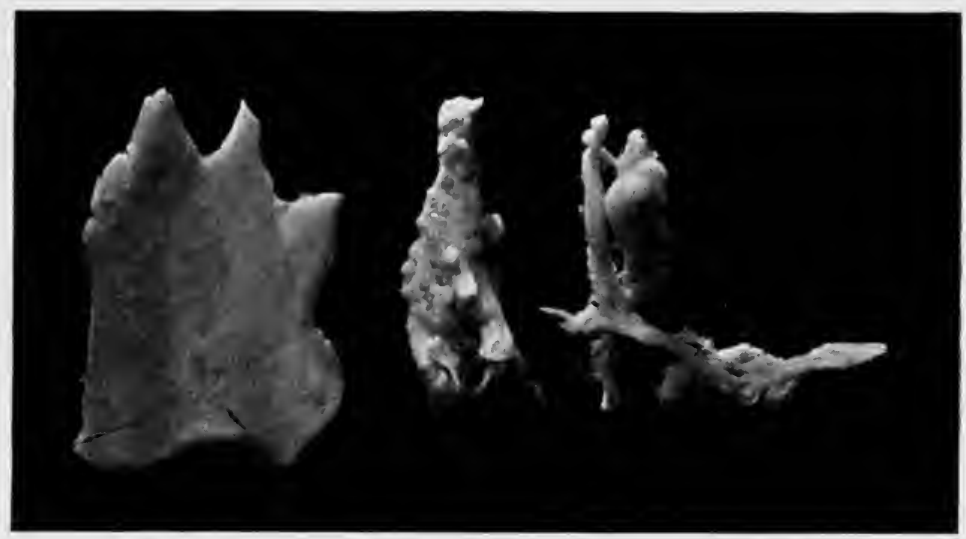

Fig. 23. Structures removed from the same knee-joint. That on the left is a portion of a much flattened internal semilunar cartilage which had become displaced. The middle one is a portion of the thickened synovial membrane, with papillæ developing, while the specimen on the right is the much thickened ligamenta alaria, with a pedunculated cartilaginous body attached. A history of the case is given in the text. From a photograph somewhat enlarged.

of one of his internal semilunar cartilages early in life and had suffered afterwards from many attacks of relapsing synovitis.

On the left of the figure the structure represents a portion of the detached cartilage. It is much wider from side to side than is natural. The flattening-out process has been produced by the cartilage having been from time to time caught between the 


\section{SEQUEL E OF SPRAINS}

articular surfaces of the bones during the movements of the joint. In its structure are signs of commencing calcification.

The middle figure represents a portion of the synovial membrane much thickened and hypertrophied, showing numerous papillæ of different sizes growing from its visceral surface.

The figure on the right of this depicts the remains of the ligamenta alaria removed from the intercondyloid region of the joint, also at the time of operation. It may be noticed that the alar ligaments have become very fibrous and so drawn out as to represent two firm bands. Attached to one of these ligaments were two oval highly polished pedunculated bodies, one of which is shown in situ.

The case is interesting and unique as affording an opportunity for studying the consequences which may follow on the neglect of a knee-joint in which a detached semilunar cartilage is present.

The movements of the piece of semilunar cartilage which had become separated and loose within the joint for years gave rise to a very chronic form of synovitis. As a result the synovial membrane became much thickened and roughened. On its visceral surface the papillæ increased in size, so that the interior of the whole joint was studded with numerous minute papillæ in various stages of development. The ligamenta alaria had become attenuated and converted into thin fibrous bands; and from the inter-condyloid attachments of these two well-formed pedunculated bodies were to be seen. Their pedicles had not yet become sufficiently long to allow of their being nipped by the 
bones in the normal movements of the joint. One of these oval bodies was cut and examined microscopically. It presented histologically a well-defined mass of fibrous tissue in the centre of which were several rapidly dividing hyaline cartilage cells. Thus, in these pictures taken of specimens from the same joint, it is possible to demonstrate the formation of the two chief types of loose bodies in joints-the one a portion of a semilunar cartilage that had become detached and free through violence; the other type, the result of true growth and development, taking place in the tissues of the joint as a result of the undue stimulation of a constant and prolonged irritation.

It is furthermore quite conceivable that had this individual at some time contracted tuberculosis, the deposition of the homogeneous or laminated fibrinous bodies ('rice bodies') would have been superadded so as to complete the list of the three commoner varieties of loose bodies with which we are familiar.

The detached cartilage, it should also be noted, shows distinct signs of calcareous deposition, a possible forerunner of other osteo-arthritic changes presumably about to begin. This case is an important one when considered from an etiological standpoint. It embodies in itself and illustrates practically the views held by various writers on the subject.

While, however, the etiology and pathology of loose bodies afford ample opportunity for speculation and difficulty, the clinical features and symptoms, as well as the treatment, are straightforward and simple. 
The symptoms of a loose body caught or nipped between the bones of a joint are very characteristic and will be considered more in detail under the heading of displaced semilunar cartilages (p. 147 et seq.). They are essentially those of sprain and arise from the same cause, viz. overstretching of ligaments. Pain, swelling, and temporary loss of function accompany in varying degrees every such accident.

When a body becomes completely and freely movable as a result of detachment, the patient is usually soon made aware of its peregrinations, and the structure may be observed to move from place to place. When, however, it is moored to its place by means of a pedicle or other attachment it remains in the same locality, while the pain and tenderness experienced on pressure or during manipulations become more localised.

The resulting synovitis is apt to assume a very chronic and recurrent type.

Treatment consists in the removal of the loose body by opening the joint. This should be done only under the strictest aseptic principles. Formerly every device short of opening directly into the joint was practised, so dangerous was the operation and so great the risks of disaster. The surgeon of to-day should no longer have qualms, providing his technique is modern and he has full confidence in his assistants. Before opening such a joint, one subjected to an obstinate and chronic synovitis, it is advisable that the patient should be kept in bed for a few days to diminish the amount of effusion and allow ample time for the limb to be properly prepared. 


\section{CHAPTER IV}

\section{INDIVIDUAL ARTICULAR SPRAINS}

\section{OF the UPPER EXTREMity}

The Sterno-clavicular joint, by reason of its great natural mobility, is seldom sprained or dislocated; occasionally, however, the surgeon meets with such a case. When it does occur, it is generally the result of a pulling form of violence applied so that the extended arm is forcibly dragged outwards and backwards away from its sternal attachment.

The most common injury is a partial separation of the inter-articular cartilage from the capsular ligament anteriorly. Pain is often considerable, increased with every movement of the limb, and the joint is exquisitely tender to the touch. The effusion into the capsule is probably for the most part blood, as there is often considerable extravasation in the immediate neighbourhood of the joint, spreading far and wide over the upper part of the chest. Diagnosis presents little difficulty; the bones usually retain their normal relationship.

Treatment consists in bandaging the limb securely to the side of the chest, with the fore-arm supported in a sling for three or four days. After this time, massage and movements, active and passive, should be commenced and continued up to the time of complete recovery. Three weeks is the average duration of treatment. 
The Acromio-clavicular joint, unlike the sternoclavicular, is more restricted in its range of movements, and for this reason is more frequently sprained. The ligaments being strong, are seldom torn across, but are frequently overstretched, and as there is as a rule little or no swelling, the injury partakes more of the nature of a strain than a sprain. It may result either from a direct blow or from a heavy fall upon the shoulder. The point of greatest tenderness corresponds with the line of the joint over the ridge of the meniscus. Pain is seldom severe unless undue pressure is brought to bear upon the joint itself. The diagnosis from fracture of the outer end of the clavicle or of the acromion is often one of extreme difficulty and may sometimes be quite impossible without the assistance of radiography. The history of a fall upon the shoulder is rather in favour of a sprain, while a direct blow is usually followed by a fracture. An exact diagnosis is of much importance, both in the relation to prognosis and treatment. These sprains, when neglected, are peculiarly liable to result in a painful joint, and complete cure, even when the injury is treated on rational lines, is often delayed for some unexplained reason. It has frequently been noticed by the writer that a sprain at the acromio-clavicular joint will often take twice as long to get well as will a fracture of the clavicle. This is particularly noticeable in dealing with young athletes, persons who are usually in the best possible health ; in them, complete recovery takes place more quickly from almost any other sprain in the body than it does from the one in question. It may be that in many cases in the past, on which his 
experience is mainly based, they were really cases of unrecognized sprain-fracture, made worse by early movements and massage. A more extensive use of radiography in the future may alter this view. It is his custom, in all cases of accident insurance, when compensation is sought, to give a guarded prognosis as to the date of recovery. Treatment is best carried out by bandaging the arm to the side with the forearm in a sling, leaving the injured part fully exposed to view. Rubbing should not to commenced till ' the bruising is coming out', usually after the third day. Movements of the joint had better be postponed for a week, for immediate movements do more harm than good. If all the pain has disappeared in three weeks the case may be considered to have done well. There is seldom any after-stiffness or muscle-wasting. If the pain should return, especially if it be nocturnal, rheumatic or gouty remedies are indicated ; blistering may be tried in obstinate cases.

Sprains of the shoulder-joint. This joint, depending as it does for its stability mainly upon the strength of the surrounding muscles and tendons, and to a less degree upon that of its ligaments, is much more predisposed to dislocation than sprain. Sprains of the shoulder-joint are rare, and result more from the laceration of the tissues arranged around the joint than from tearing of the ligaments themselves. The capsular ligament is so thin, and possesses so little inherent strength, that it is easily torn. This is seldom possible, however, unless the surrounding muscles are caught unawares or are lacerated before the strain is brought to bear upon it. The whole region becomes swollen and tender 
to the touch, the point of the greatest intensity corresponding with the upper attachment of the long head of the biceps, immediately below the acromion process. The tenderness may be acute along the whole line of the biceps tendon. Any manipulation whereby the head of the humerus is made to move in the glenoid cavity will cause pain, as will any stretching force applied either to the tendons or ligaments. The swelling is occasionally considerable; not only is the capacious capsule itself full of effusion, but extensive bleeding takes place into the tissues around. The hæmorrhage is mainly from the vessels of supply to the muscles and tendons, that have been torn across, and the blood may be diffused into the loose tissue of the axilla, or into the inter-muscular spaces, or it may become collected into definite hæmatomata between the fibres of the deltoid muscle, or in the large bursa (sub-deltoid) situated between this muscle and the capsule of the joint. The swelling often renders the differential diagnosis a matter of considerable difficulty, so that an examination with an anæsthetic or radiography is often not only advisable but indispensable. This particular injury, especially when there has been much bruising, is very liable to be followed by prolonged pain and stiffness in the upper arm, with considerable crippling. The muscles around, and which move the joint, notably the deltoid, supra- and infra-spinatus, not infrequently undergo rapid atrophy. Neuralgic pain, usually attributed to neuritis and referred to the neighbourhood of the deltoid insertion, is often complained of. It probably results from the circumflex nerve or its WHTELIOCKE 


\section{2 INDIVIDUAL ARTICULAR SPRAINS}

branches being caught and pressed upon in the matting together of the muscles by the extravasated material outside the capsule of the joint. As soon as an exact diagnosis has been arrived at, and all complications excluded, treatment should be begun. When the surgeon is called to see a case soon after the injury, his first aim should be to check or limit the amount of extravasation, for the quantity of blood poured out has an important influence in the later management. This may be best effected by the early application of elastic compression. The axilla should be well padded and the whole region of the joint smoothly covered with cotton wadding, over which a firm spica bandage is applied. When however the case is not seen until later, at the time when the swelling has reached its maximum and the patient is unable either to abduct or to adduct his arm, or indeed to move it in any way without causing intense suffering, warm applications are very comforting and may relieve the tension to a certain extent. Flannels wrung out of warm lead lotion or a decoction of poppy-heads may be wrapped around for an hour or two. These warm applications find their particular use with a greater certainty in sprains of the shoulder than perhaps in any other joint. In a short time, say in two or three hours, elastic pressure may be substituted. By this time the patient will have lost some of that intense aching and the sense of stretching which so often accompany rapid swelling, and will more readily submit to the application of a bandage. Whenever it is feasible, during the early stages of treatment, the elastic pressure should be removed and re-applied 
twice at least in the day. If there is a large collection of blood in the axilla, or the sub-deltoid bursa is over-distended and painful, so that a bandage cannot be worn with any comfort, it is often advisable to aspirate and draw off the fluid parts of the blood as a preliminary to the application of pressure. In a few cases of very severe contusion of the shoulder, in which large hæmatoma were present, it has been found of great advantage to make an incision through the deltoid, and to turn out all the clots in its substance or in the bursa beneath it. Sprains of this joint are always, when severe, accompanied by much bruising, so that the treatment of sprain is largely the treatment of contusion. Massage should never be commenced until the swelling has distinctly begun to subside, though gentle movements of a voluntary type may be allowed from the first. In making passive movements, care should be taken not to rub the head of the humerus against the glenoid cavity. The shoulder-girdle should be fixed by an assistant while the surgeon goes gently through the various movements, commencing with flexion and extension, followed by rotation, leaving abduction till the last. As most sprains occur when the arm is in the abducted and extended position, it stands to reason that the lower part of the capsule suffers the greatest damage. Abduction is generally resisted for a considerable time, and for two reasons. When the arm is raised, the sub-deltoid bursa is pressed upon and squeezed, and the biceps tendon is made to slip in its groove ; both of these movements are painful. The pain of abduction may sometimes be partially overcome by simply employing 


\section{INDIVIDUAL ARTICULAR SPRAINS}

stroking massage for a few minutes immediately before the passive movements are tried. Passive movements find their greatest value in the treatment of sprained and contused shoulders. Voluntary movements, however slight, must be made from the earliest stages, and continued until complete restoration to function is established. Unless this detail is carefully attended to, rapid wasting, not only of the deltoid but of all the muscles on the proximal side of the joint, is likely to supervene. Even when the arm is bandaged to the side, movements in the fore-arm and hand should be made, for this allows nervous impulses to pass to all the muscles of the arm. The bad results ensuing from neglected sprains and contusions are due to an insufficient appreciation by surgeons of the fact that it is the matting together of the tissues surrounding the joint, and not the tearing of the ligaments themselves, that is responsible for the stiffness and pain afterwards. Every effort should therefore be made throughout the whole charge of the case to prevent or break down these adhesions by movements and massage. A shoulder that has become stiff and painful through neglect and indifferent treatment should in the first place be thoroughly moved under an anæsthetic, so as to have all the adhesions completely broken down, and from that time onwards treated as a recent sprain.

In very extreme cases, even after the stiffness and pain have gone, muscular atrophy may never be completely recovered from, and a weakened joint remains. The only treatment which seems of real value in cases exhibiting considerable muscle-wasting is a continued and systematic course of muscular 
exercises. It is usually recommended that exercises, arranged so that the muscles act against resistance (as are carried out by means of weights and pulleys or with the aid of elastic cords or bands), should be practised at a gymnasium under an instructor. Without underestimating the value of any form or forms of active voluntary movements, whenever they are performed regularly and systematically, the writer thinks that much can be done by the patient himself at home. In the earlier stages, at any rate, a gymnasium is quite unnecessary, nor is any definite apparatus required. All that is wanted is that the individual should be clearly instructed as to what movements are required and what it is that these are expected to do for him. He will be able to prevent or even overcome atrophy by the simple expedient of extending both arms, clenching his fists firmly (whereby all the muscles of the arm and forearm are made tense), then very slowly and gradually flexing the wrists, then the elbows, at the same time that he lifts both arms as high as possible above his head. This should be done three or four times in a day, actual fatigue being always avoided. The system of making the muscles contract firmly without giving them too much work, as is often done when dumb-bells are used, is all that is required for developing healthy muscles during athletic training. The same principle will be found equally successful when applied as a means of regenerating muscles that have atrophied; it has the additional advantage of not requiring any special apparatus.

The elbow-joint is very frequently sprained as the result of a fall on to the outstretched hand, or 


\section{INDIVIDUAL ARTICULAR SPRAINS}

from a twist of the fore-arm. The damage is generally greatest to the external lateral and orbicular ligaments, but any of the ligaments may be injured.

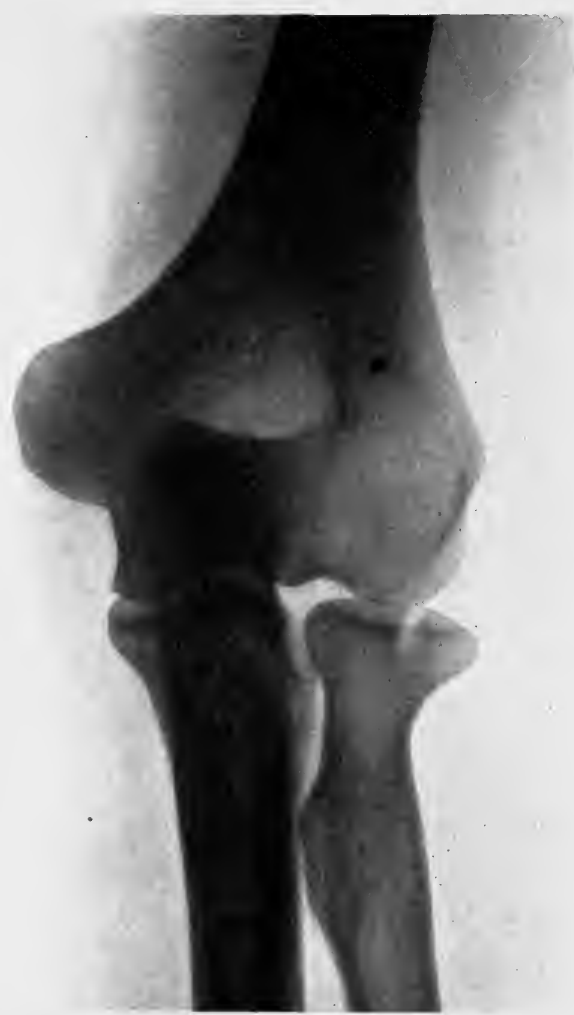

FIG. 24 is a radiogram of a fracture-sprain at the elbow. The head of the radius was fissured as the result of a fall down a bank upon the outstretched arm. The symptoms were merely those of simple sprain; a fracture was suspected from a slight difficulty in complete supination, some weeks after the accident. From a lady aged 36 .

Effusion takes place into the synovial cavity, and the joint becomes rounded off and swollen, while the natural hollows around it are filled up and disappear. 


\section{INDIVIDUAL ARTICULAR SPRAINS}

The diagnosis must be made from fracture and dislocation, and, in children, from partial separation of an epiphysis. In sprain, the bony points about the joint retain their normal relationship; this fact is usually sufficient to establish the diagnosis ; should

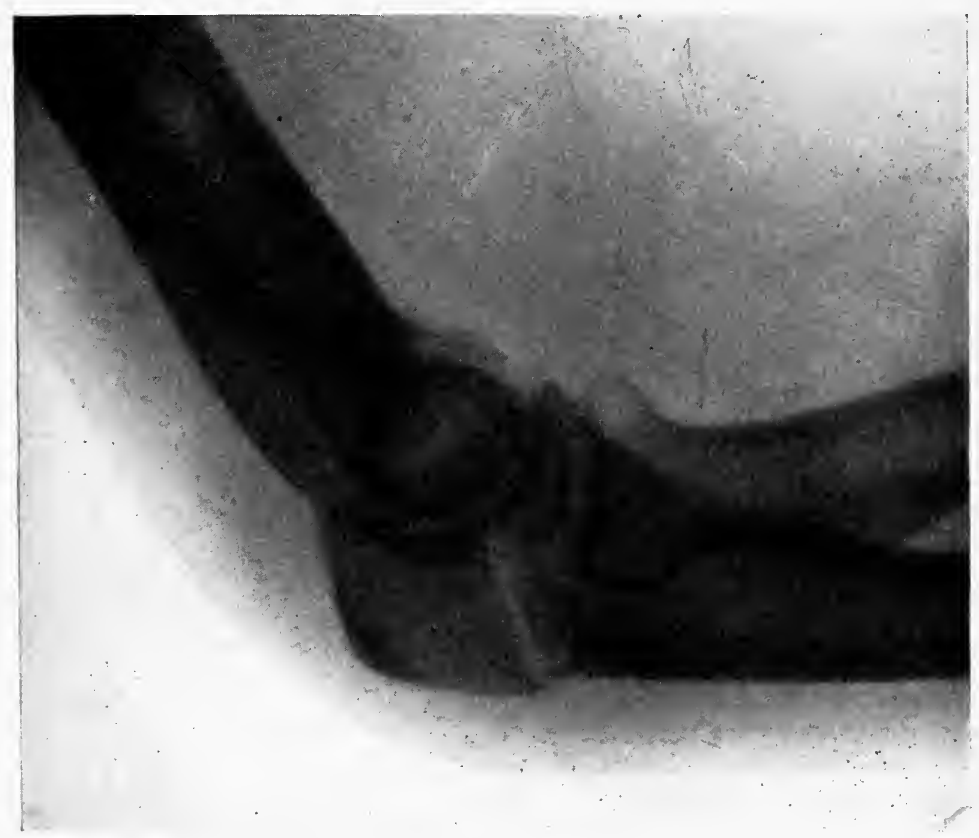

FIG. 25 is a radiogram of a fracture-sprain at the elbow, produced by a fall and treated as a simple sprain until its true nature was detected by radiography (Mr. J. Keogh Murphy's case).

there however be any doubt, radioscopy will be useful. Of partial separation of an epiphysis in a child it is often difficult to make certain, even with the aid of the $\mathrm{X}$-rays. A very tender spot in the line of an epiphysis will often put the observer on his guard. Treatment resolves itself into elastic compression 


\section{INDIVIDUAL ARTICULAR SPRAINS}

until all effusion has disappeared, followed by early movements and massage. Complete recovery may be expected in from fifteen to seventeen days when there is no fracture or complication.

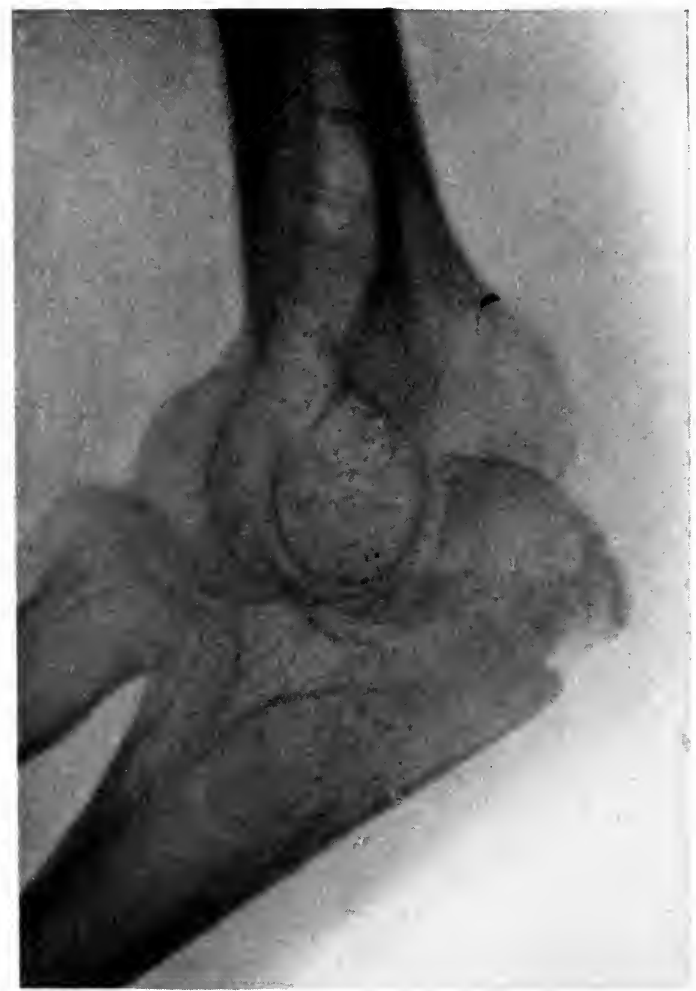

FIG. 26. Radiogram of an impasted fracture of the olecranon. Thought at first to be, and treated as, a simple sprain. Stiffness resulted.

'Dislocation by Elongation,' or 'Pulled Elbow'. A subluxation of the head of the radius, though technically a dislocation, may be considered clinically as a sprain. It is met with in children under six, results from the child being lifted or dragged 
by the hand, and is usually on the left side. It is of not infrequent occurrence. The pulling and twisting of the fore-arm disengages the head of the radius from the orbicular ligament. The peculiar attitude struck by the fore-arm is due to the edge of the ligament passing between the bones, and in this way preventing rotation. The child complains of pain, and is unable to use its arm; the fore-arm remains halfway between pronation and supination. When an attempt at supination is made, severe pain is complained of at the elbow. There is remarkably little deformity, although the head of the radius may be seen projecting in front of the joint. The wrist is also painful, for it is strained by the same lifting process, and there may be even effusion into this joint, with swelling. The child is usually brought complaining of the pain at its wrist rather than at the elbow, and it is only after an examination has been made that the double injury is discovered. As this is, in the main, a true sprain of the wrist, it has been considered right to place the injury amongst the sprains. Reduction frequently takes place spontaneously, and the arm resumes its wonted mobility. Whenever in a child under six both the elbow- and wrist-joints are found full of effusion, this particular injury may be suspected.

Treatment. A general anæsthetic is usually necessary, for the manipulations are painful. The elbow is flexed, and, while traction is made on the fore-arm, pronation and supination are alternately practised. When reduction has been effected, both the joints should be strapped with plaster, and the fore-arm 
placed and kept in a sling. Massage over the strapping may be commenced from the first. In the course of about ten days the movements in both joints should be painless and free.

'Sprained Elbow' is a term used for a painful condition of the muscles arising from both condyles

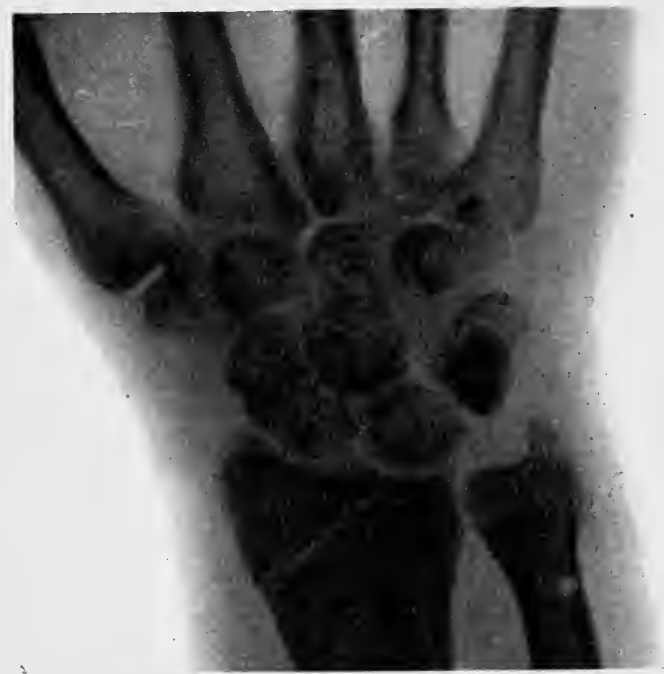

FIG. 27. Fracture through lower end of radius without displacement, resulting from bang of swinging gate against the outstretched hand of a young lady while riding. Symptoms only of sprain; real condition detected by radiography. (Mr. Edwin Dening's case.)

of the humerus and produced by excessive or faulty exercise of these muscles, chiefly of the supinator longus and the extensores carpi radiales longiores et breviores. Many forms of exercise, such as lawntennis, fly-fishing, golf, and handling a coachingwhip, may produce it. The pain radiates from the condyles downwards into the fore-arm. As it is in reality and more properly a muscular sprain it will 
INDIVIDUAL ARTICULAR SPRAINS 91

be referred to when treating of muscular sprains. The inter-muscular septa and fasciæ of the fore-arm are occasionally overstretched or torn, as evinced by

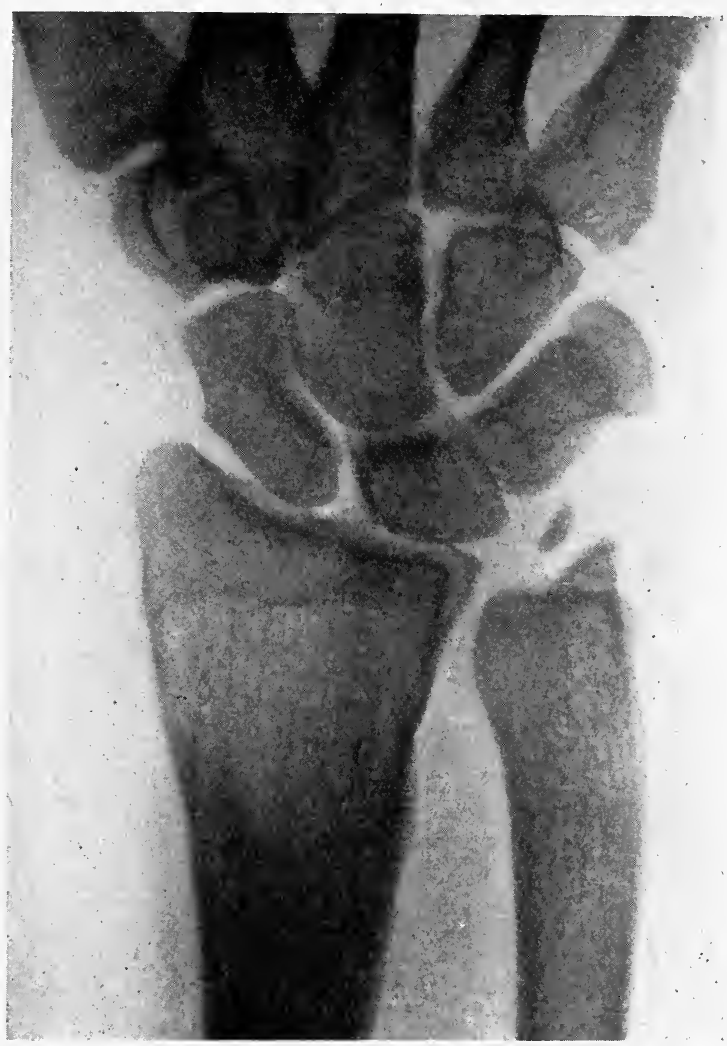

FIG. 28. Fracture of the tip of styloid process of the ulna. From symptoms was regarded as simple sprain. Fracture detected late in the treatment by the X-rays.

the exceptional presence of a muscle hernia, and the marked discoloration of the skin which follows on later.

Sprain of the wrist. Simple sprain is a very 


\section{INDIVIDUAL ARTICULAR SPRAINS}

common injury and results from a fall upon the hand and various twisting forms of violence incidental to athletic exercises. Pain and swelling, with loss of function, are the chief symptoms. The swelling is often considerable, and renders the diagnosis diffi-

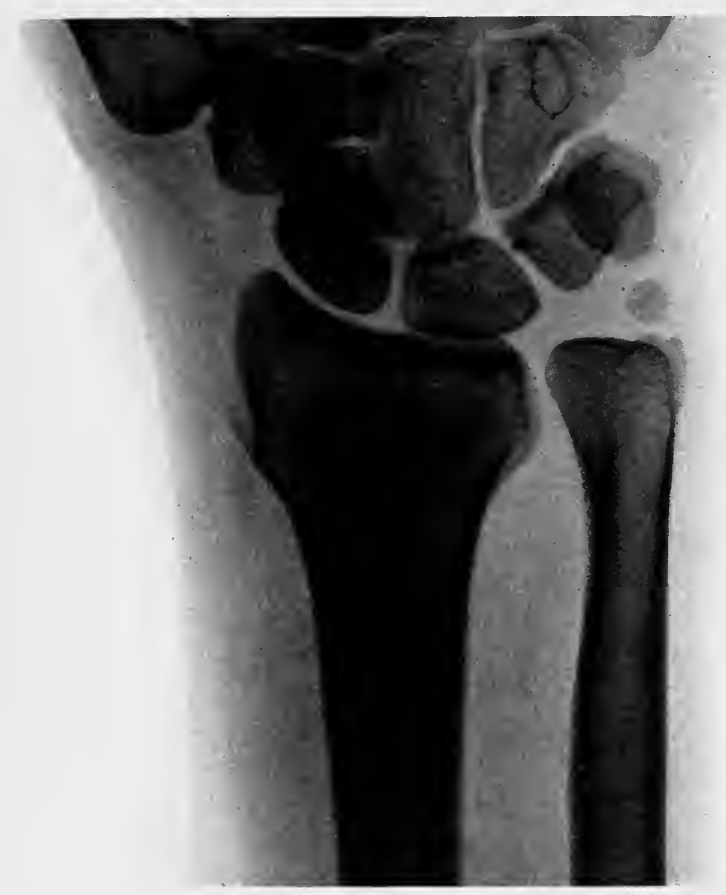

FIG. 29. Impacted fracture of lower end of radius without displacement. Fracture suspected from severe pain when supination was attempted.

cult. This has to be made from Colles's fracture, and other fractures at the lower end of the radius, especially when the fragments are impacted or without displacement, as in Figs. 27 and 29, from fracture of the styloid processes of the radius or ulna, Fig. 28, 
separation of the radiocarpal epiphysis, Fig. 30, from dislocation of the radiocarpal articulation, and from fracture of one of the carpal bones. Mr. E. W. H. Shenton ${ }^{1}$ has drawn attention to a diagnostic sign

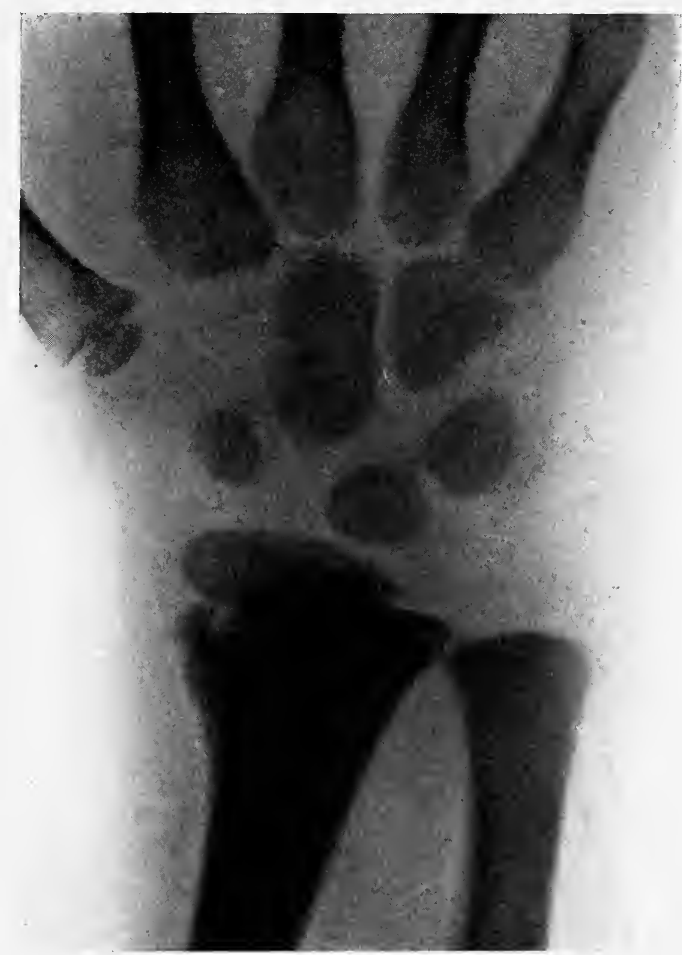

Fig. 30. Sprain-dislocation at wrist, with partial displacement outwards of the hand with the lower epiphysis of the radius, and showing some tearing of the periosteum of the diaphysis. Symptoms those of sprain.

of much value in the differential diagnosis of injuries in the region of the wrist. He states that if the hand is turned so that the thumb is uppermost, either by the operator or the patient himself, in Colles's frac1 Guy's Hospital Gazette, Nov. 27, 1909, p. 488. 


\section{INDIVIDUAL ARTICULAR SPRAINS}

ture the movement is never executed without evidence of acute pain. If the movement can be performed with ease or moderate discomfort Colles's fracture

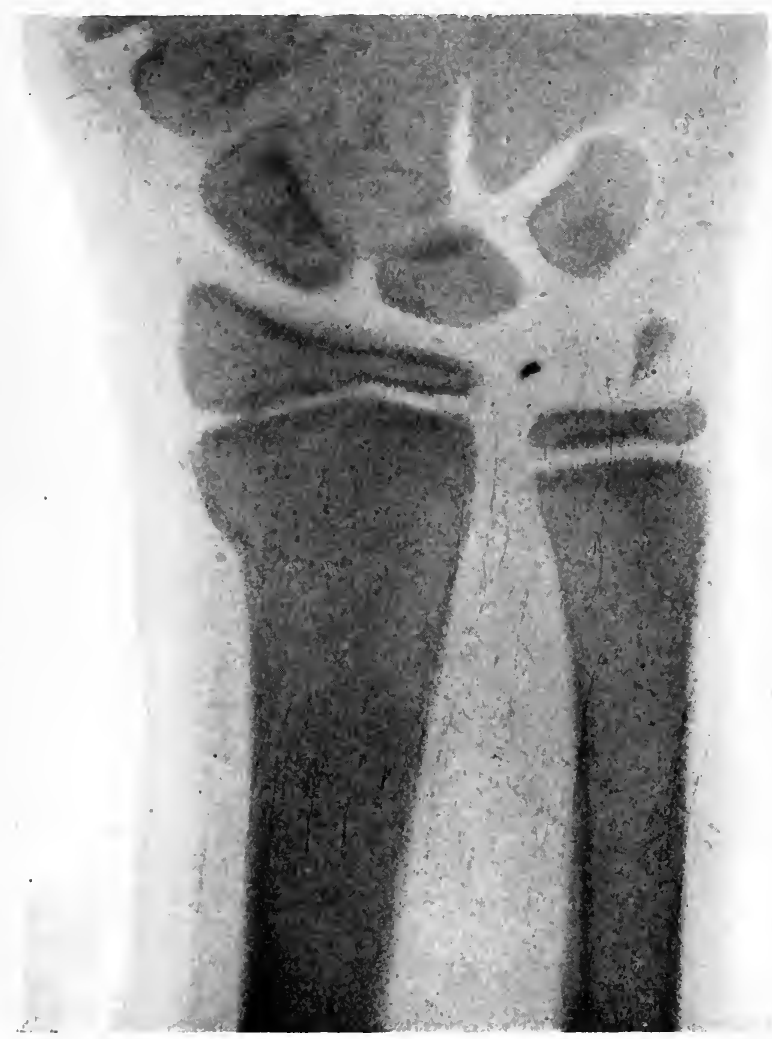

Fig. 31. Complicated sprain of wrist. Greenstick fracture through lower end of the radius without displacement; also detachment of the tip of the epiphysis at the lower end of ulna. Exact condition determined only by the help of radiography. Originally thought to be simple sprain.

is not present. The observation was verified by the use of the X-rays.

In other fractures at the lower end of the radius, 


\section{INDIVIDUAL ARTICULAR SPRAINS 95}

which cannot properly be described as Colles's fracture, as in Figs. 27, 29, 31, and in which there is neither deformity nor displacement of the fragments to be detected, the movements of pronation and supination are likewise always painful. In every severe

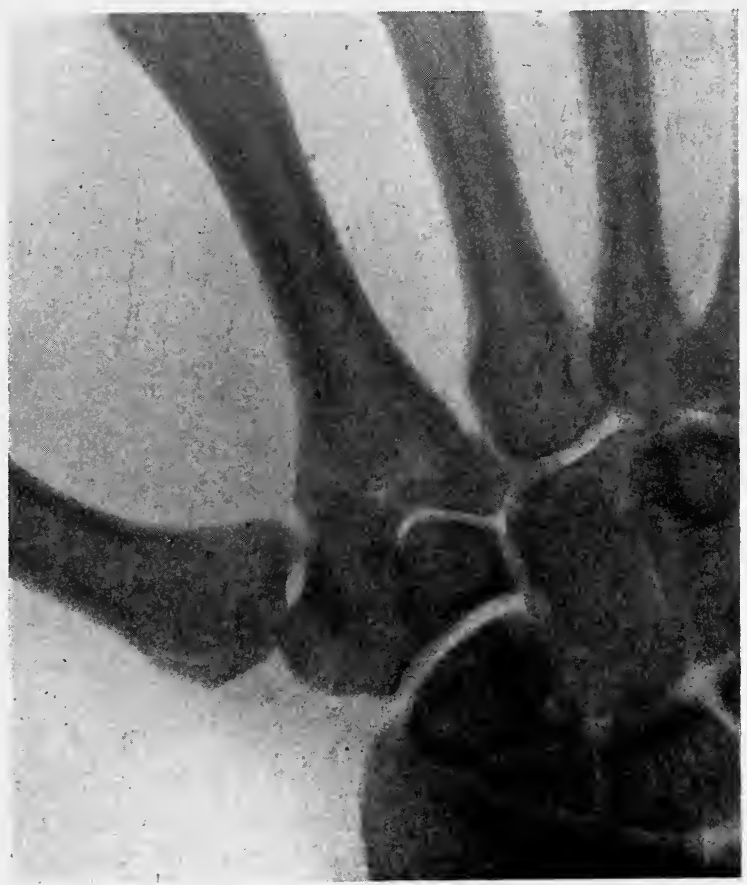

FIG. 32. Fracture of scaphoid. Fracture-sprain of wrist; symptoms only of simple sprain; result of misplaced blow with the fist while boxing. The bone is split into three pieces.

sprain of the wrist when the movements of pronation and supination are distinctly painful a fracture should be suspected.

It is of the greatest importance to ascertain whether there is a fracture of one of the carpal bones. The normal relationship of the styloid processes to 
96 INDIVIDUAL ARTICULAR SPRAINS

the other bony points is of importance, for they remain normal in simple sprains.

The swelling is most marked anteriorly and posteriorly along the line of the joint, and, besides this, there is usually some effusion running up the sheath

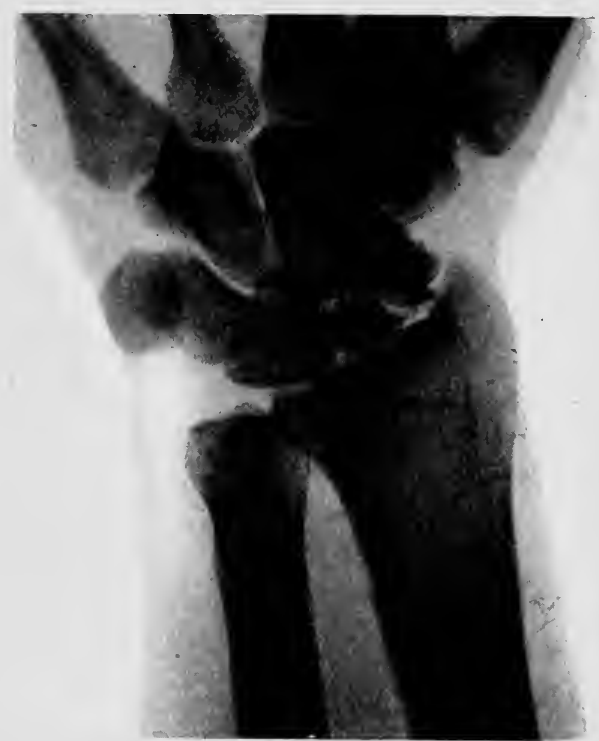

Fic. 33. Fracture of scaphoid bone of right wrist, result of a Rugby football accident. Patient had been treated by movements and massage on the assumption that it was a case of simple sprain. For details vide text, pp. 98-101.

of the tendons into the fore-arm and down into the hand.

Treatment consists of elastic pressure, strapping, early movements, with or without massage. It is next to impossible to determine the presence or absence of a fracture through one of the carpal bones without the $\mathrm{X}$-rays.

Fracture of the scaphoid bone. The writer, during 


\section{INDIVIDUAL ARTICULAR SPRAINS}

the year 1908, met with three cases of fracture of the scaphoid bone, all of which had at first been regarded as ordinary simple sprains. ${ }^{1}$ Two of these had occurred some weeks before the writer saw them; the third was seen directly after the accident. A

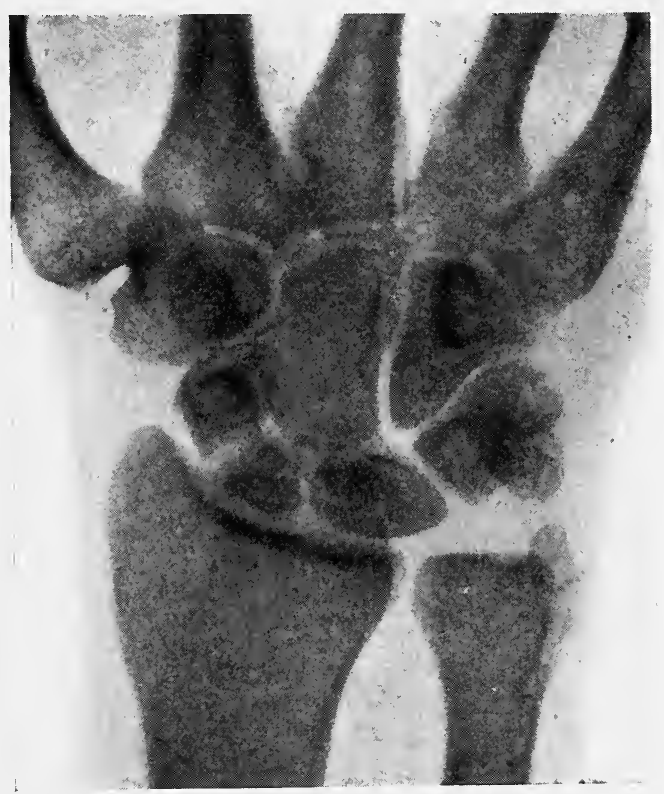

Fig. 34. Fracture of scaphoid bone of carpus, from Association football goalkeeper's accident. Symptoms same as those of sprain; diagnosis only possible by X-rays. Treated by rest for fourteen days, and later by movements.

correct diagnosis was made, in all three cases, by means of radiography, without which in his opinion it could not have been established.

As the condition is a comparatively rare one, and the treatment so dependent upon an accurate

1 Since this was written four additional cases have been treated. One of them is illustrated by Fig. 35 .

WHITELOCKE

H 
diagnosis, it has been thought wise to record the cases somewhat fully.

The accompanying radiographs show very distinctly in each case the presence of a fracture through the scaphoid bone. The patients were all undergraduates, and distinguished athletes. The injuries were produced by different forms of athletic exercise and in different ways.

In the first case the patient was a heavy-weight boxer, and while delivering a blow struck the end of his clenched fist so as to drive it somewhat to the radial side and almost directly backwards on to the wrist, the muscles of the fore-arm being at the time firmly contracted.

The second case was that of a Rugby football player who fell while running with the ball.

The third was the University Association football goalkeeper, who had his hand forcibly wrenched backwards in endeavouring to keep the ball out of goal. The clinical symptoms were remarkably similar in all the cases, so that a description of one will serve for all. The Rugby football player (Mr. W. Elliot Stanford, of New College) was good enough to write out the details of the history and symptoms of his accident. It seems a more or less accurate account of what occurred before and after he came under personal observation, and is here given in his own words.

' I was playing Rugby football in the Parks at Oxford, on October 15, 1907, and while running along at top speed I was tackled hard and low, and fell violently towards my right side. My idea is, that in falling, I got my right arm into such a position that my hand was wrenched back by 
the whole weight of my falling body ; but of this I cannot be quite certain. It might very well have been that the wrist was doubled up underneath me as I fell, and not really forced backwards; but the former was my impression at the time. The effect was very painful, and the hand and fore-arm were practically paralysed for the time being. Immediately after the game I returned to college and had a hot bath, the effect of which probably was to counteract the tendency to swell. The wrist had swelled, but not to any great extent, and become stiff, and of course I could not attempt to use it at all. Immediately after the bath I had the wrist bandaged with a cooling liniment, and this treatment I continued for the next few days. The swelling reached its maximum the day after the accident, and then gradually decreased, until the only sign of swelling was a. little thickness in the part of the joint immediately above the ball of the thumb, which remained for some time after. Not knowing that there was any fracture as well as the sprain, I did not as a rule have the wrist bandaged; I attempted to loosen it by massage, and succeeded to a certain extent. I could not use the hand except merely to hold with the fingers when there was no actual strain on the wrist. I could move my hand backwards and forwards to a small extent and the scope of the movement gradually increased up to a certain point. During the first fortnight I twice jarred the wrist rather badly. On each occasion it was a sudden strain backwards, and after each of these jars the wrist was worse for a time. Any sudden movement of the wrist, however slight, hurt it. After the first fortnight I could flex the wrist forward, that is towards the palm, until it was bent almost as far as it could be normally ; but the process was rather painful. I could bend the hand backwards to a very limited extend, and any effort to press it further backwards was painful if the fingers were bent, but if the fingers were stretched out, I could force the hand back at will, by a muscular contraction of the fore-arm, and although it could not be bent any further than when the 


\section{INDIVIDUAL ARTICULAR SPRAINS}

fingers were flexed, yet, strange to say, it caused no pain, but on the application of external pressure by pressing it back with the other hand it became painful. Since the $\mathrm{X}$-rays examination showed that there had been a fracture, I have been wearing the strapping you recommended, around the wrist, and using it as little as possible. The joint is now a little stronger, but still gives pain on making the movements described above, and I can still feel slight pain on pressing the fingers of the left hand on the thumb side of the right wrist, particularly underneath the part of the joint which is directly above the ball of the thumb ; the joint frequently grates and cracks when I bend it.'

These details were written láte in November, or six weeks after the accident. On July 29, 1908, he wrote the following additional account :

' I have been exercising my damaged wrist lately, and at present it seems to be troubling me less than it did a month or two ago. There is now very little stiffness or pain in palmar and ulnar flexion, if the movements are steady, but any sudden movement, particularly if unexpected and therefore unresisted, still produces a sharp pain in the region of the fractured bone. From a position in which the fingers are in the same line with the fore-arm it is impossible to flex the joint radially, either by passive or active movement, and any effort to do so is attended with pain of a sharp and burning nature, when the movement is sudden and unexpected, if for instance I catch my thumb in putting my hand into my pocket. I cannot bend my hand back any further than I could months ago ; it resists both active and passive movements beyond a certain angle, about $5^{\circ}$ from the normal limit of dorsal flexion; but while attempts to force it further are painful, active force produces very little pain. This restriction I find a great handicap, as it prevents me from pressing away with my palm unless I keep my elbow bent. The palmar surface of the wrist is still slightly thickened below the root of the thumb, and if I 


\section{INDIVIDUAL ARTICULAR SPRAINS 101.}

let my hand hang and press against a certain small spot on the inside of the small hard lump, a slight click is produced which hurts slightly the first time, but repetition does not produce further pain. I ought to have said, in referring to the palmar flexion of the wrist, that the freedom of flexion is

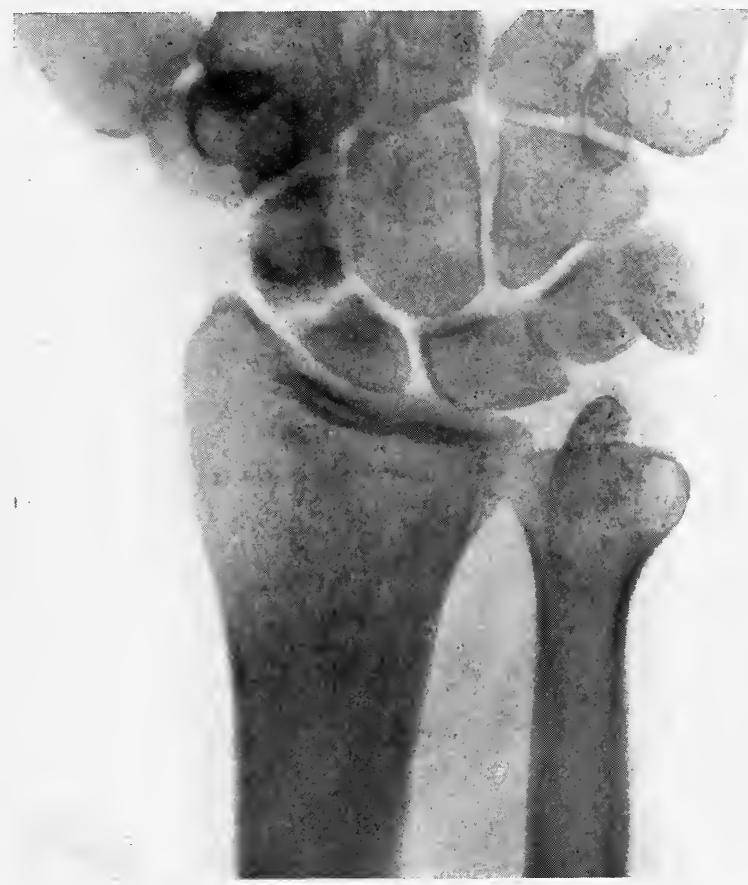

FrG. 35. Radiogram showing complete fracture of scaphoid bone of left wrist as result of fall on to the hand.

less on the thumb side than on the other, with the consequence that there seems to be a certain amount of ulnar flexion involved. The wrist cracks and grates to a certain extent when I exercise it.'

The history and progress of this case are, as was stated before, very similar to that of the boxer's. In 


\section{INDIVIDUAL ARTICULAR SPRAINS}

each of these two cases the injury was, in the first instance, regarded as a simple sprain and treated by early and continued movement, and resulted in the same unsatisfactory manner. In the goalkeeper's case the symptoms at first were similar, but as an immediate diagnosis was made with the help of radiography, instead of early passive movements and massage being used, the wrist was strapped and the arm worn in a sling for a fortnight. No massage was allowed until after this time, although each day gentle voluntary movements of the fingers were practised while the wrist was firmly grasped and supported. The result was that the case was completely cured, so that the goalkeeper was able to play football in six weeks from the time of his injury, bony union having probably taken place. In the former two cases the fragments remained detached, so that each carpus now virtually contains nine bones instead of eight. Until by long-continued movements the fragments of the scaphoid shall become sufficiently polished, or so covered by hyaline cartilage, that they move smoothly upon one another, it is likely that stiffness, pain, and limited movements will continue.

This group of cases admirably exemplifies the statement made elsewhere, that sprains with fracture must be treated in a way quite different from that used for simple sprains. Massage and early passive movements are not only useless, but harmful. Such a sprain should be allowed complete rest for at least ten days, any subsequent stiffness and tendency to atrophy being prevented by allowing 


\section{INDIVIDUAL ARTICULAR SPRAINS 103}

gentle and judicious voluntary movements in the fingers, and it is only in the later stages that massage is really of service.

The patients are all well-known athletes; at the

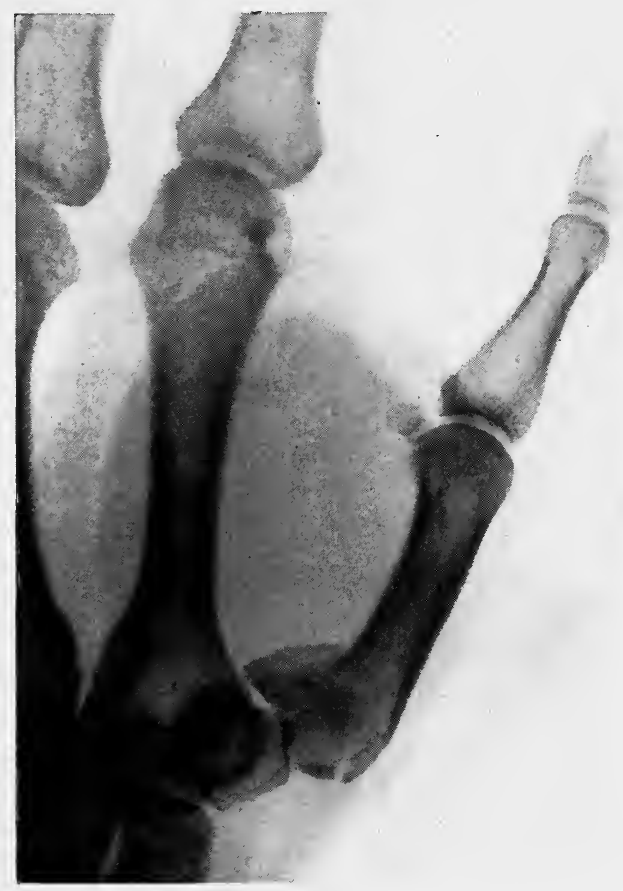

FIG. 36. Fracture at base of metacarpal bone of the thumb, resulting from a fall on to the abducted and outstretched thumb. No crepitation could be felt, and the symptoms were only those of sprain. Bennett's fracture.

University; the boxer was a heavy-weight champion, and the goalkeeper represented his University against Cambridge six weeks after the accident.

The three accompanying radiographs (Figs. 32, 33, 34) show plainly the precise degree of fracture in 


\section{INDIVIDUAL ARTICULAR SPRAINS}

each case. In Fig. 35 the fracture resulted from a fall on to the hand.

Sprains of the thumb are very frequently met with in athletes. They result from any form of

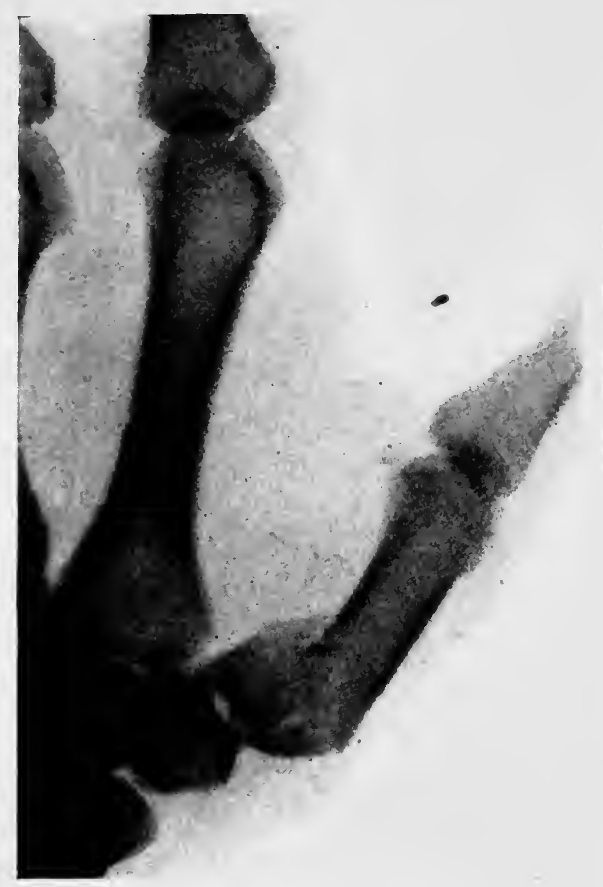

FIG. 37. Fracture at base of metacarpal of the thumb. Treated formerly as simple strain. Radiogram shows impaction of fragment, Bennett's fracture so-called, dorsal view.

violence sufficient to overstretch or tear the ligaments either of the carpo-metacarpal or the metacarpo-phalangeal joints. The symptoms are pain, swelling and stiffness. The pain is increased by any form of movement. It must be distinguished from the fracture occurring at the base of the metacarpal 
bone first described by Bennett of Dublin, and which has since taken his name, also from fractures of the body of the metacarpal bone itself. The accompanying photograph is from a case in which there was a linear fracture through the metacarpal bone and which was at first regarded as one of simple

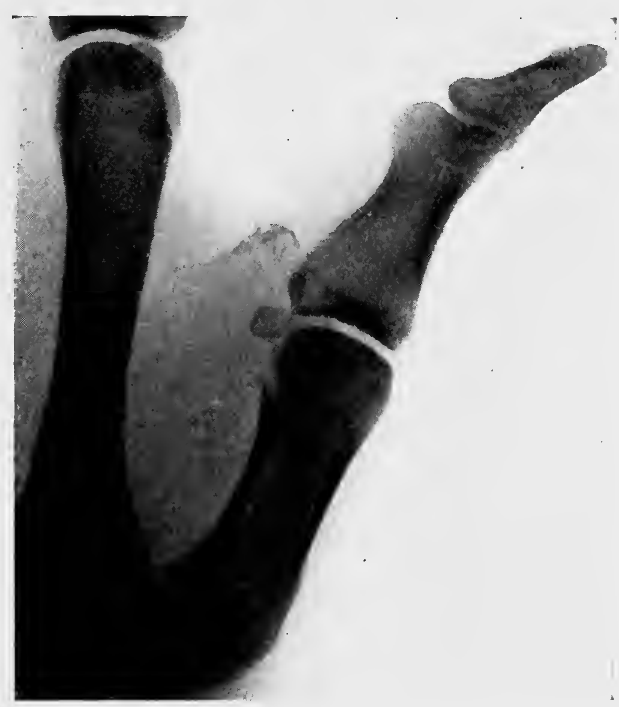

Fig. 38. Linear fracture of metacarpal bone of the thumb. No displacement. Carpo-metacarpal and metacarpo-phalangeal joints both full of effusion. Had been treated as sprain until X-ray revealed presence of fracture. Result of accident at cricket.

sprain. In this case there was effusion, both into the carpo-metacarpal and the metacarpo-phalangeal joints. It occurred in the person of the University wicket-keeper, and was the result of a direct blow from a cricket ball. No symptoms of fracture could be detected, and it was only after the usual methods of treatment for sprain had failed to remove the 


\section{INDIVIDUAL ARTICULAR SPRAINS}

symptoms that a fracture was suspected and an X-ray photograph taken. By referring to the picture the split in the bone may be seen quite distinctly, though there is no evident displacement of the fragments.

The treatment of simple sprain of the thumb is by means of plaster strapping, with movements and massage from the very earliest time. Stiffness is likely to remain unless the movements are maintained for some time after all pain has disappeared. Sprain-fractures, and sprains with fracture, on the other hand, must be treated as fractures, and not by indiscriminate movement; at first rest is essential.

'Drop-top,' or 'mallet-finger', is a sprain of the attachments of the long extensors of the thumb and fingers to the dorsum of the terminal phalanges. It is met with in cricketers, chiefly wicket-keepers, and results from a cricket ball striking the tips of the fingers when they are extended as in the act of catching. The digits are forcibly flexed and the attachments of the extensor tendons are either considerably overstretched or completely separated from their phalangeal attachments. The terminal phalanx droops forward, and although it may be completely restored by manipulation, the patient is unable to extend it at will. The resulting disfigurement is often unsightly. The writer knows an old professional wicket-keeper who has one thumb and six fingers crippled in this way. The accompanying photograph is from the hand of a schoolboy in whose case there was an oblique fracture through the phalanx, a fracture-sprain, and not a 


\section{INDIVIDUAL ARTICULAR SPRAINS 107}

true sprain as at first supposed. The deformity at first sight closely resembles drop-top, and but for the $\mathrm{X}$-rays might have been mistaken for that condition.

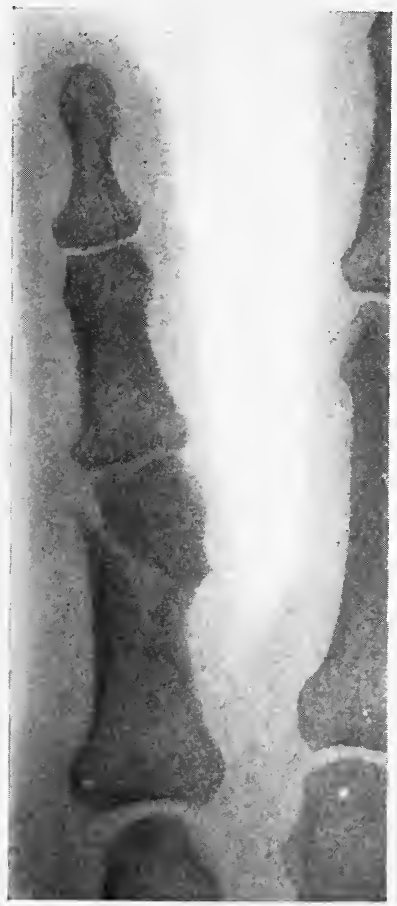

39

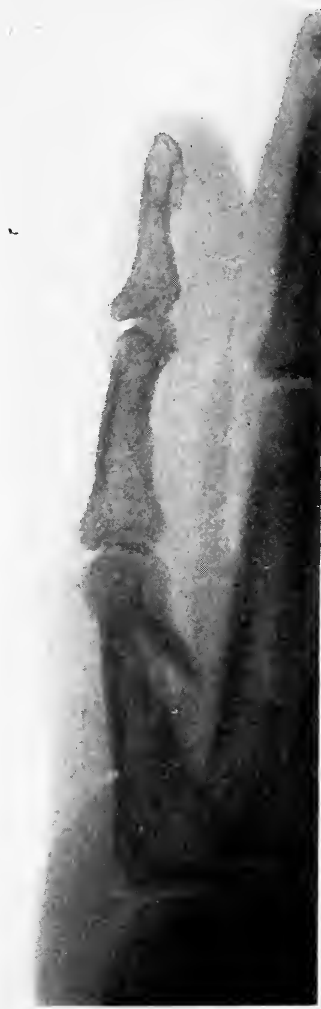

40

FIG. 39. Radiogram of fractured phalanx of index finger, the result of a fall. Had been treated as a sprain by movements and massage. Without X-rays the fracture could not be detected, as the displacement was slight and the swelling marked.

Frg. 40. Profile view of a greenstick fracture of the first phalanx of finger. Symptoms those of simple sprain.

The treatment is to fix the finger to a dorsal splint extending beyond its tip, and to strap the phalanx 


\section{INDIVIDUAL ARTICULAR SPRAINS}

to it with plaster. Movement and massage should not be used for at least fourteen days. The result is often disappointing, and in the case of the thumb

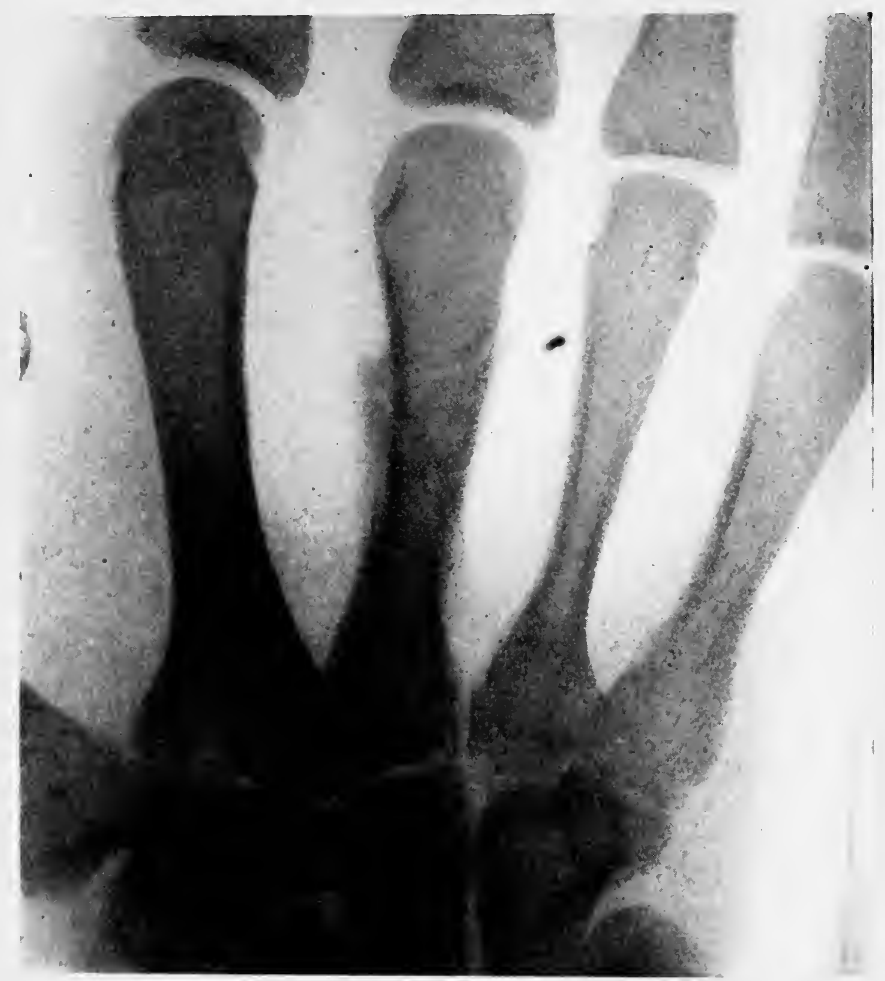

FIG. 41. Fracture (greenstick) of metacarpal bone of middle finger. No displacement, but showing callus. Had been treated as a sprain of hand, resulting from a fall.

causes considerable inconvenience. On a few occasions the writer has operated and stitched the end of the detached tendon to the periosteum, having taken the tendon as far forward as possible, then fixed the finger in the hyper-extended position by 
means of a moulded splint, which was worn for about three weeks. The result in four of the seven cases was good; in the remaining three there was marked improvement, though the power of extension was never complete. The method, however, is useful, and may be recommended where the deformity is a source of annoyance to the individual. In two of the cases the patients were anxious to enter the public services, one the Army, the other the Indian Civil Service; they were both finally admitted.

Fracture of the first phalanx of the little finger is often thought to be a sprain. Without radiography it is often exceedingly difficult to detect a separation of the upper epiphysis of this bone. It is a common injury with schoolboys, and is sometimes caused by catching the tip of the finger in parts of the clothing, or in their pushing against one another with outstretched fingers. The treatment is best carried out by strapping the finger to its neighbour for a few days until the pain and swelling have subsided, and then using massage. Definite deformity may remain, and the pain occasionally lasts a considerable time.

\section{Sprains of the Lower Extremity}

Sprains of the hip are comparatively rare and result from any form of violence which causes overstretching or laceration of the ligaments. There is often considerable effusion into the joint, and the whole articulation is tender on pressure, and painful when moved, however slightly. The limb assumes the position of slight flexion, with abduction and 


\section{INDIVIDUAL ARTICULAR SPRAINS}

external rotation, that which, owing to the distension of the capsule, affords the greatest ease. On measurement no alteration in the relation of the bony points to one another will be found, and there is no shortening. At first, especially when there is much swelling and bruising around the joint and the tenderness is marked, it is exceedingly difficult to exclude fracture. In such cases the wise plan is to treat the injury as one of fracture by putting the patient to bed and watching for other symptoms.

The prognosis of sprains and contusion of the hip in persons of middle age and onwards must be guarded. There are many cases on record in which it would appear that a simple strain was the determining factor for osteo-arthritic changes, which afterwards made the joint absolutely stiff. The gluteal muscles are liable to considerable wasting.

As to treatment, the individual should be put to bed and kept there for some days ; no splint is necessary, and none should be allowed. Friction massage of the thigh and buttock may be commenced early. Elastic pressure is not convenient. Active movements of the foot and bending of the knee and ankle should be encouraged all the time, and as the pain diminishes the patient may be allowed to turn himself in bed and to flex the joint itself. These movements are most beneficial when repeated several times in the day. Passive movements and kneading massage are of more service late in the case, beginning usually about the tenth day, or about the time that the patient should first be allowed to get out of bed. The movements and rubbing must be continued regularly and carried out systematically for at least a fort- 
night after the patient is able to walk. The muscles waste rapidly and become weak and therefore require strict attention during the whole period of treatment, and on to the time of complete recovery. Joints that are treated by fixation are the ones most prone to osteo-arthritis and permanent stiffness.

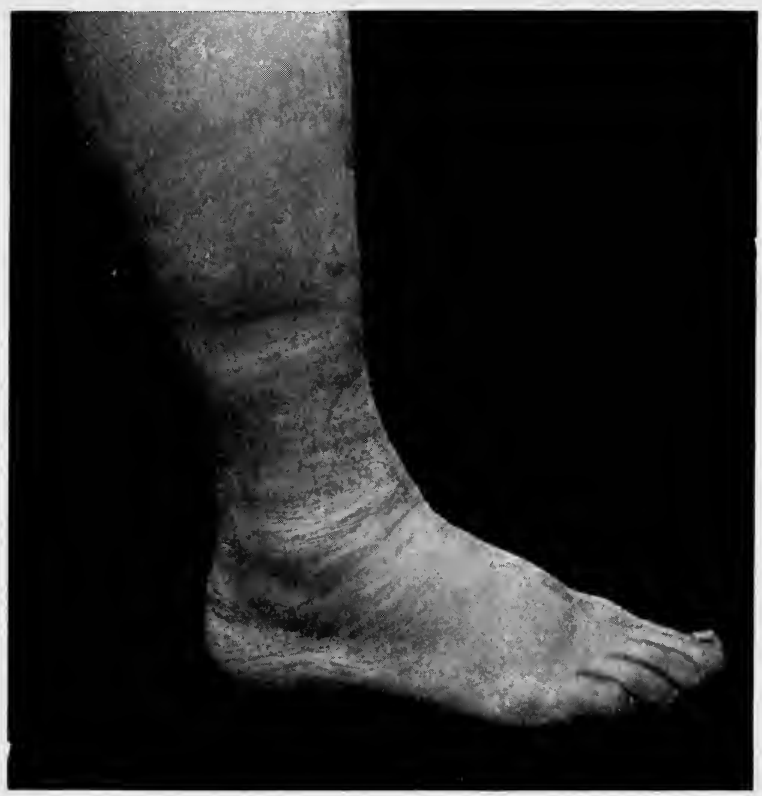

Fig. 42. To illustrate a severely sprained ankle. The photograph was taken to show the effect of an improperly applied elastic bandage. The indentation seen is the result of tightly bandaging the limb without sufficient wadding. Note the characteristic appearance of bruising around the joint.

Contusions of the hip or its neighbourhood should be treated in the same way as sprains, i. e. by rest in the early stages, followed later by massage and movements.

Sprains of the knee are treated of in Chapter V. 


\section{INDIVIDUAL ARTICULAR SPRAINS}

external rotation, that which, owing to the distension of the capsule, affords the greatest ease. On measurement no alteration in the relation of the bony points to one another will be found, and there is no shortening. At first, especially when there is much swelling and bruising around the joint and the tenderness is marked, it is exceedingly difficult to exclude fracture. In such cases the wise plan is to treat the injury as one of fracture by putting the patient to bed and watching for other symptoms.

The prognosis of sprains and contusion of the hip in persons of middle age and onwards must be guarded. There are many cases on record in which it would appear that a simple strain was the determining factor for osteo-arthritic changes, which afterwards made the joint absolutely stiff. The gluteal muscles are liable to considerable wasting.

As to treatment, the individual should be put to bed and kept there for some days ; no splint is necessary, and none should be allowed. Friction massage of the thigh and buttock may be commenced early. Elastic pressure is not convenient. Active movements of the foot and bending of the knee and ankle should be encouraged all the time, and as the pain diminishes the patient may be allowed to turn himself in bed and to flex the joint itself. These movements are most beneficial when repeated several times in the day. Passive movements and kneading massage are of more service late in the case, beginning usually about the tenth day, or about the time that the patient should first be allowed to get out of bed. The movements and rubbing must be continued regularly and carried out systematically for at least a fort- 
night after the patient is able to walk. The muscles waste rapidly and become weak and therefore require strict attention during the whole period of treatment, and on to the time of complete recovery. Joints that are treated by fixation are the ones most prone to osteo-arthritis and permanent stiffness.

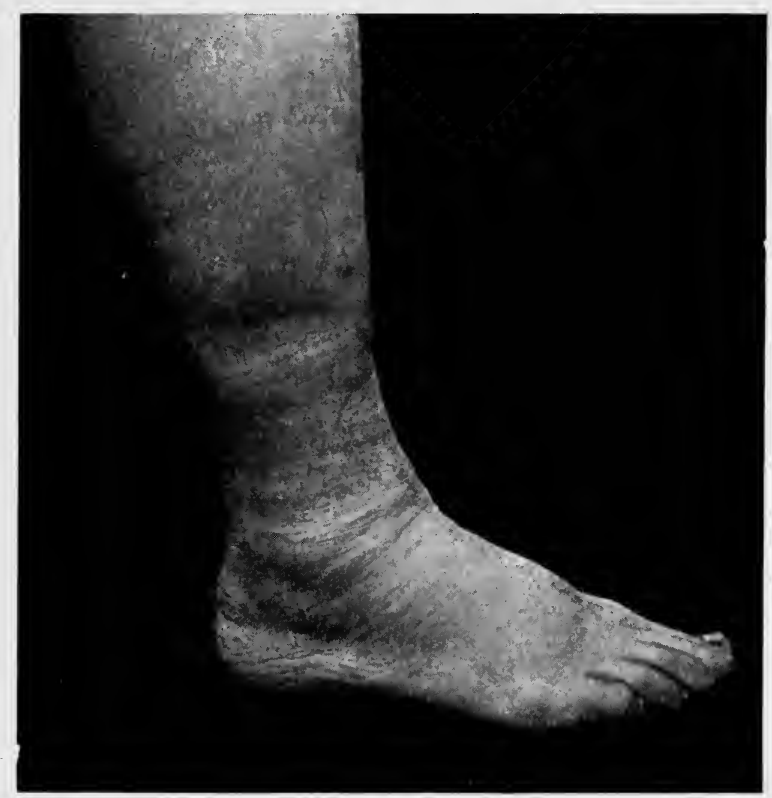

Fig. 42. To illustrate a severely sprained ankle. The photograph was taken to show the effect of an improperly applied elastic bandage. The indentation seen is the result of tightly bandaging the limb without sufficient wadding. Note the characteristic appearance of bruising around the joint.

Contusions of the hip or its neighbourhood should be treated in the same way as sprains, i. e. by rest in the early stages, followed later by massage and movements.

Sprains of the knee are treated of in Chapter V. 


\section{INDIVIDUAL ARTICULAR SPRAINS}

siderable subcutaneous extravasation of blood, on some occasions extending from the ball of the little toe to half-way up the leg, following the lines of the tendon-sheaths.

The most suitable treatment for strains when there is little or no effusion is strapping. This should be applied in overlapping strips and laid on smoothly. Beginning at the ball of the toes, the strips of plaster should be crossed in front of the joint so as to cover it completely and give firm support. (For special method of strapping vide Chapter X, Fig. 83, p. 256.) In the slighter cases the patient may be allowed to stand as soon as the plaster has been applied. Movements in the toes with slight flexion and extension at the ankle ought to be encouraged daily, even if a certain amount of pain is experienced. Whenever the strapping becomes loosened it should be immediately re-applied and continued to be worn until all pain has disappeared and the strength and movements in the joint are fully restored. Massage may or may not be needed.

When there is effusion into the capsule and extravasation into the tissues around the malleoli and into the tendon-sheaths, no plan succeeds so well as elastic pressure. This should be applied as soon as possible, the sooner the better, and removed and re-applied two or three times a day. Massage and passive movements must not be allowed until all the effusion within the capsule has gone; after this they may be practised with much advantage. Active movements may be commenced from the very first and continued throughout the treatment, but the weight of the body ought not to be put upon the 
ligaments for at least ten days after the accident. The prognosis is good in all simple sprains, providing the patient is not allowed to walk too soon. A neglected sprain renders the joint either painful or weak, or both. If walking is permitted before the fluid has disappeared, or before the ligaments are sufficiently strong to support the weight, a relapse is almost certain to follow. Few things are more tiresome, both to the patient and surgeon, than a weak and painful ankle.

When walking is first begun it is well that a bandage should be used as a support during the day and removed at bedtime. An elastic anklet may be worn on the first two or three occasions on which violent exercise is indulged in, but should be removed as soon after as possible. Strong elastic bandages are to be condemned, for when worn continuously they produce weakness in the joint.

Cases that relapse after an interval of days or even weeks, when there is absence of fresh injury, are generally due to rheumatism or gout. An acute attack of gout or rheumatism may supervene on an injury to the ankle. These constitutional complications should be met by the remedies appropriate to each.

'Metatarsal pain' may be classified with sprains. It is of a chronic or subacute nature, and is dependent upon a stretching of ligaments and perhaps tendons. As its name denotes, it is a painful condition met with in the sole of the foot at the metatarso-phalangeal joints. It may usually be traced to the wearing of tight and improper boots with narrow soles and high heels. The toes are squeezed together and the whole 


\section{INDIVIDUAL ARTICULAR SPRAINS}

weight of the body is thrown forwards and downwards on to their bases. The transverse ligaments which bind the heads of the metatarsals together are unduly stretched dorsally as one or other metatarsal bone becomes displaced beneath its neighbour. This stretching force is brought into play whenever the foot is put forward in the act of walking. Sometimes there is a limited swelling over the metatarsophalangeal joints. The anterior part of the sole is tender to the touch, and is often very painful when the individual first attempts to walk after removal of the shoes.

The treatment consists in substituting a broadsoled boot with flat heels for the improper one previously worn, and rubbing the foot night and morning with some stimulating embrocation. The pain is sometimes so severe as to make walking impossible. If shampooing does not succeed in relieving the pain, wrenching the foot under an anæsthetic, as a preliminary to rubbing, may succeed. The deep friction produced by the application of an electric vibrating machine has sometimes been of signal service in intractable cases.

The tarso-metatarsal joint of the great toe is occasionally sprained during the performance of athletic exercises. The symptoms and diagnosis present nothing unusual, and the treatment is on general lines.

Fracture of the tuberosity of the fifth metatarsal bone is by no means uncommon, and used formerly to be frequently mistaken for sprain of the foot. It results from a violent twist of the outer side of the foot, as in wrestling when barefooted, or from landing 
awkwardly in jumping. The diagnosis is sometimes difficult without the $\mathrm{X}$-rays, as there may be very little displacement of the fragments. It is a painful affection, and may cause lameness for several weeks. The treatment consists of rest and plaster strapping. Massage over the strapping will often relieve the pain and reduce the swelling in the early stages.

Sprains of the trunk. Besides the muscular sprains of the trunk, which will be dealt with in a succeeding chapter, we will here mention the

Sprains of the sides. In violent falls and twists of the body the ribs are frequently pulled asunder or squeezed together in a forcible way. The result is a condition in which the intercostal muscles are overstretched, and become painful with almost any movement of the trunk and with every deep inspiration. There may or may not be tenderness on palpation. Swelling is seldom conspicuous, though a considerable amount of contusion is not unknown. The diagnosis is made from a fractured rib by auscultation and the use of the X-rays. In cases of fracture the stethoscope will generally elicit crepitus. The pain, though often acute and considerable early in the case, usually becomes much diminished in three or four days. The treatment in the early stage consists of accurate strapping with plaster as for a broken rib. The plaster should at all times embrace at least three-fourths of the circumference of the chest and be retained for about eight days. Massage may be applied over the strapping, and begun early, as it often tends to diminish pain. Chronic pains of a neuralgic type may persist for weeks or even months after such an 


\section{INDIVIDUAL ARTICULAR SPRAINS}

injury. Patients of middle life and later, especially those of a gouty predisposition, are most liable to the neuralgic condition, nevertheless, young persons are not wholly exempt.

Sacro-coccygeal sprain. This is a troublesome affection, and may result from a variety of causes, the most usual being from falls on to the lower part of the spine, whereby the ligaments of the coccyx are overstretched or partially torn. The ligaments of the sacro-iliac joints may be injured from the jarring at these joints produced by heavy falls on to one or other tuberosity of the ischium or on to the end of the spine. Falls in the hunting, polo, or football fields have been known to produce it. The symptoms are pain, usually much increased on any attempt being made to rise from the sitting posture to the erect, or to sit again after having stood for some little time. Tenderness, often acute, is complained of over the whole or most of the lower region of the spine and buttocks. There may or may not be swelling; it is usual to find none. The diagnosis from dislocation or fracture of the coccyx is made by the absence or presence of pain in defæcation. In fracture or dislocation of the coccyx there is always pain during this act. A finger passed into the bowel will also readily discover a dislocation or fracture of the coccyx.

The early treatment consists in reducing any displacement of the coccyx should one be present (this is done by levering it into position by means of a finger inserted into the rectum, with the aid of an anæsthetic if necessary), then of putting the patient to bed for a few days. The recumbent position is 
necessary to procure rest as well as to relieve painful defæcation. Should the pain be severe, aspirin may be used, and, when absolutely necessary, even morphia. Warm applications to the perineum and lower dorsum are often appreciated. Later, massage and electrical vibrations will be helpful in clearing up any deep effusions. The prognosis must be guarded, for the condition known as coccygodynia, a painful neuralgic affection, may supervene and occasion much trouble, and especially is this true in neurotic subjects.

Various devices and means have from time to time been adopted to relieve the symptoms of this lastnamed affection. When the pain is very severe and the bruising extensive, the treatment as for a fractured pelvis may with advantage be adopted for the first few days, followed later by that for sprain with contusion. L. W. Ely ${ }^{1}$ has found that the ordinary treatment by palliative means, and even by resection, is highly unsatisfactory. He states that for years he has treated these cases by massage of the coccyx. The bone is held by the forefinger in the vagina and the thumb on the outside; it is then moved backwards and forwards, and the soft parts are moved about on the bone. Almost instantaneous improvement follows.

1 Journ. Amer. Med. Assoc., 1910, vol. liv, p. 968. 


\section{CHAPTER V}

\section{SPRAINS IN THE REGION OF THE}

\section{KNEE-JOINT}

Surgical anatomy. For the better understanding of the many and varied lesions met with in this part of the body, which, for convenience, are grouped together clinically as sprains, a short reference to topographical anatomy may be of service.

As the articular surfaces of the bones of the knee are simply opposed to one another without any interlocking or fixation, and as the integrity of the joint is dependent almost entirely upon the strength and arrangement of its ligaments and tendons, it is peculiarly liable to sprain. Almost every ligament and tendon, as well as the synovial membrane, may be at some time or other overstretched or lacerated.

The capsular ligament is strengthened and supported in various directions by the tendons which are inserted into it and pass over and around it. It envelops the whole articulation and encloses the ends of bone as it were in a tube. Additional support is afforded by the blending of its fibres with those of the other ligaments in its immediate vicinity.

It is lined by a synovial membrane of great laxity and extent, which is folded or pleated in various directions and gives off folds which pass inwards between the bones, completely envelops the interarticular or crucial ligaments, and for the most part 
surrounds the upper and lower surfaces of the interarticular fibro-cartilages. It is almost invariably injured when the capsule is overstretched or torn.

The patellar ligament and tendon of the quadriceps extensor represent together a powerful anterior ligament, its lateral expansion blending intimately with the capsule on either side.

The posterior ligament is also of very great strength, and is made up of bundles of fibres passing in almost every direction and reinforced here and there by strips from the tendons at the back and sides of the joint.

The internal lateral is a powerful ligament, flat and ribbon-like, passing obliquely downwards and slightly forwards from the inner surface of the internal condyle of the femur to the inner surface of the tibia in direct continuation of the line of attachment of the adductor magnus tendon to the tubercle of the internal condyle of the femur. The lower -attachment extends well below the inner tuberosity of the tibia. It consists of two parts, a superficial and deep. The superficial is about $1 \frac{1}{2}$ inches wide and is attached above and below to the femur and tibia respectively. The deep portion is as it were an offshoot of the superficial, and, passing deeply, is attached to the convex border or base of the internal semilunar cartilage at its middle, as well as to the capsular ligament in front and behind this point.

The long external lateral ligament is a cord-like structure of fair strength passing almost parallel with the internal ligament, attached above to the external condyle of the femur, and below to the 
head of the fibula. It is separated from the external semilunar cartilage by the popliteus tendon which intervenes. This tendon grooves the outer border of the semilunar cartilage deeply and gives it support.

The crucial ligaments are two, the anterior external and the posterior internal. These ligaments cross one another and bind the femur to the tibia very firmly. They are placed between the condyles of the femur entirely within the joint, are almost completely enveloped by the synovial membrane, are of great strength, and are only torn as the result of extreme violence.

The inter-articular fibro-cartilages are two, the internal larger and the external smaller. Without going into anything like a full anatomical description of these structures, it is necessary that the reader's attention should be directed to certain facts in relation to them : facts which explain the occurrence and the frequency, or otherwise, of certain injuries to which they are liable. The semilunar cartilages may be regarded as the remnants of two complete inter-articular disks placed between the condyles of the femur and the upper surface of the tibia. Through successive periods of time they have become more and more worn away and atrophied towards the centre of the joint, partly owing to the vertical pressure of the weight of the body and partly to the friction produced by the rotation which normally takes place around the tibial spines in every movement of the joint, whether in flexion or extension.

As the internal condyle of the femur is larger than the external and on a lower level, and as in all movements it is held more closely applied to the 
tibia than is the external condyle, so the internal semilunar cartilage is subjected to a greater amount of grinding strain than its fellow. Is it surprising, then, that we find this cartilage more worn away than its neighbour, more attenuated, narrower from side to side, and proportionately weaker? The wearing away is more at its anterior end than at the posterior. The external cartilage is more movable and is not subjected to as much grinding between the bones as the internal. On rare occasions it is found as a complete disk, or with but a small central foramen. The anterior half of the internal cartilage is weaker than any other part of either cartilage.

As regards the attachments of the horns of the cartilages it will be seen that the posterior ones have very firm attachments to the tibia; especially is this the case with the external cartilage, which is reinforced by two fibrous slips. One of these arises from the posterior crucial ligament and the other passes from it to the outer surface of the internal condyle of the femur posteriorly. This latter slip is often absent. The anterior cornu of the external cartilage is also firmly bound down to the tibia while the anterior extremity of the internal cartilage varies greatly in its arrangement and strength of attachment. In some cases it is represented only by a delicate strip which passes right across in front to be attached to the front of the external cartilage, and finds no direct tibial attachment. At other times it is but loosely connected with the upper and anterior border of the tibia, and blends with the capsular ligament or the periosteum, the most common site for its attachment being to the front of 


\section{SPRAINS IN THE REGION OF}

the internal tibial spine immediately anterior to the attachment of the anterior crucial ligament.

This anatomical variation in the attachment of the anterior horn of the internal semilunar cartilage is largely responsible for the displacements and detachments so frequently met with in the anterior half of this cartilage. To a more detailed consideration of the surgical significance of this point reference will again be made when treating of the internal derangements of the knee.

The body of each cartilage is bound down to the upper edge of its respective tibial tuberosity by means of the coronary ligaments. These bands are of fair strength and are attached to the peripheral convex bases of the cartilages. The internal lateral ligament gives attachment on its deep surface to the internal cartilage, while the popliteus tendon lying in a groove in the outer border of the external cartilage assists in maintaining its position.

Sprains of the knee are comparatively common as the result of a twist or a wrench. The ligaments are overstretched or torn across partially or completely. The joint becomes much swollen through effusion into it. This effusion extends to the utmost limits of the synovial cavity so that the hollows on each side of the patella are abolished and the quadriceps tendon pushed forwards, and when the joint is full the patella is raised from the trochlear surface of the femur and made to 'float'.

The 'floating patella' may be felt to strike the femur when pressed upon suddenly. It must be mentioned, however, that this symptom of floating patella may be deceptive, and must not be too 
much depended upon in making a diagnosis. Beginners are wont to overestimate its value. Two facts are important in relation to it; firstly, the patella does not float unless there is considerable effusion, sufficient to distend the capsule more or less completely: unless this is borne in mind when the symptom is absent, slight effusions may be overlooked; and, secondly, a degree of floating is present when the synovial membrane itself is thickened and where there may be only very little fluid in the joint. This condition of things exists in certain cases of early tubercular disease and must not be mistaken for effusion.

I have known a surgeon declare that there was no fluid, because he was unable to make the patella float and rap against the femur. Manipulation of the patella in a sprain is a painful process, and a very unnecessary one for making a diagnosis.

If the surgeon, on passing his eyes critically over the natural hollows on each side of the patella in a recently injured knee, finds them obliterated, he may be certain that he has to deal with an effusion into the joint and may never need to touch the swelling to establish his diagnosis. On the inner side of the patella, at a point corresponding with the hollow referred to just now, the only structures which overlie the synovial membrane are the skin and fascia, and a thin expansion from the extensor tendon blending with the capsular ligament. The eye is all that is needed to make the diagnosis, and patients may be spared unnecessary pain if this simple fact is borne in mind.

The differential diagnosis from fracture and separa- 
tion of epiphyses is not always simple, but careful attention to the normal relationships of the bones will go far in preventing mistakes.

The point of greatest tenderness in sprains of the knee is usually at the attachment of the internal

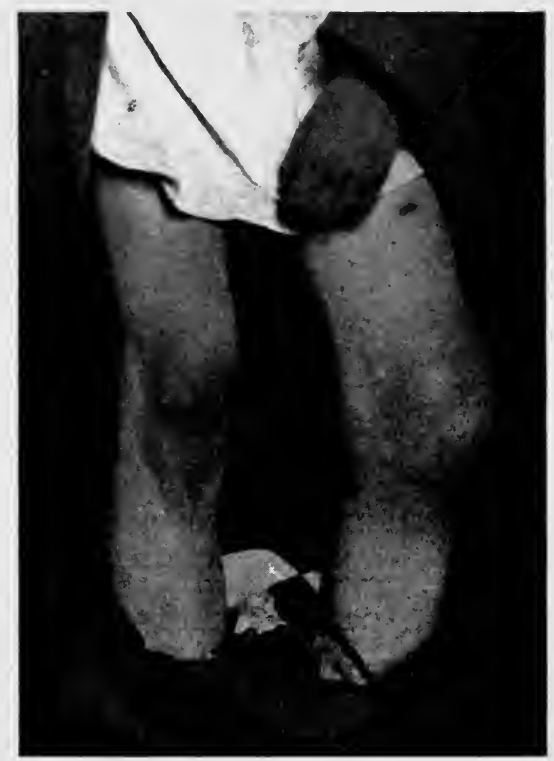

FIG. 44. Photograph from sprained knee four hours after the accident. Note the obliteration of the natural outlines of the joint when compared with the other knee. This is typical of full distension of a knee-joint with blood or synovia.

lateral ligament to the inner surface of the femoral condyle, less often over the middle of the base of the internal semilunar cartilage. When the external lateral ligament is most affected, and this is rare, the tenderness and pain are located near the head of the fibula and back of the joint.

When a sprain of the knee has been neglected or 
improperly treated, stiffness and weakness are the predominant after-effects. When there is much atrophy of the quadriceps extensor muscle and of the gluteus maximus and tensor fasciæ femoris, the capsule loses its proper support and sags. Even a slight strain to such a knee is sufficient to cause a copious effusion into the synovial cavity. To this chronic and relapsing condition the name of traumatic hydrops has been given. The frequency with which this form is met with in football players has given to it the popular name of 'footballer's knee'. In old people sooner or later these chronically distended joints are followed by symptoms of osteo-arthritis.

Treatment. In all cases of acute traumatic synovitis of the knee the patient should be confined to bed. Elastic pressure if immediately applied will relieve pain, arrest and diminish the effusion, and hasten absorption. Slight active movements in the toes and ankle may be commenced from the first; massage and passive movements not before the effusion has disappeared. Speaking generally, the quickest recoveries follow those cases that are seen early and in which the modern and rational treatment is begun from the first and carried out by the sugeon himself until all symptoms have disappeared.

Given a young healthy individual, he should be perfectly able to resume all his athletic exercises in three weeks.

In the chronic and relapsing forms often the best line of treatment is to bring about an acute attack by forced movements and manipulation under an anæsthetic, then to employ the rational régime as 
if the joint were being treated for a first attack. Massage may be needed for a considerable time and should be continued until the muscles quite regain

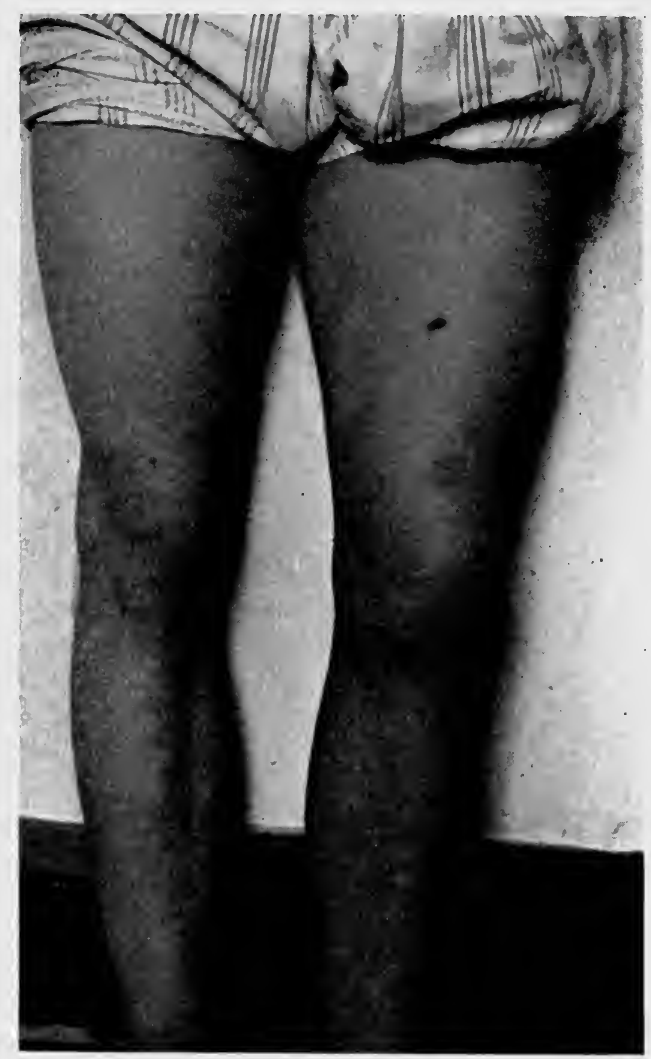

Fig. 45. Acute traumatic synovitis of the left knee-joint as result of displacement of the external semilunar cartilage. The swelling came on gradually, though quickly. The photograph was taken two hours after the accident. Note the slightly flexed position of the joint, and its general enlargement, with loss of anatomical detail when compared with its neighbour.

their strength. During all this time a non-elastic bandage may be worn for support. As soon as it is safe the bandage should be discontinued at night, 
and only used when support is actually needed. The wearing of a bandage, although of some slight support, will not be of itself sufficient to prevent recurrent injury; its presence, however, serves

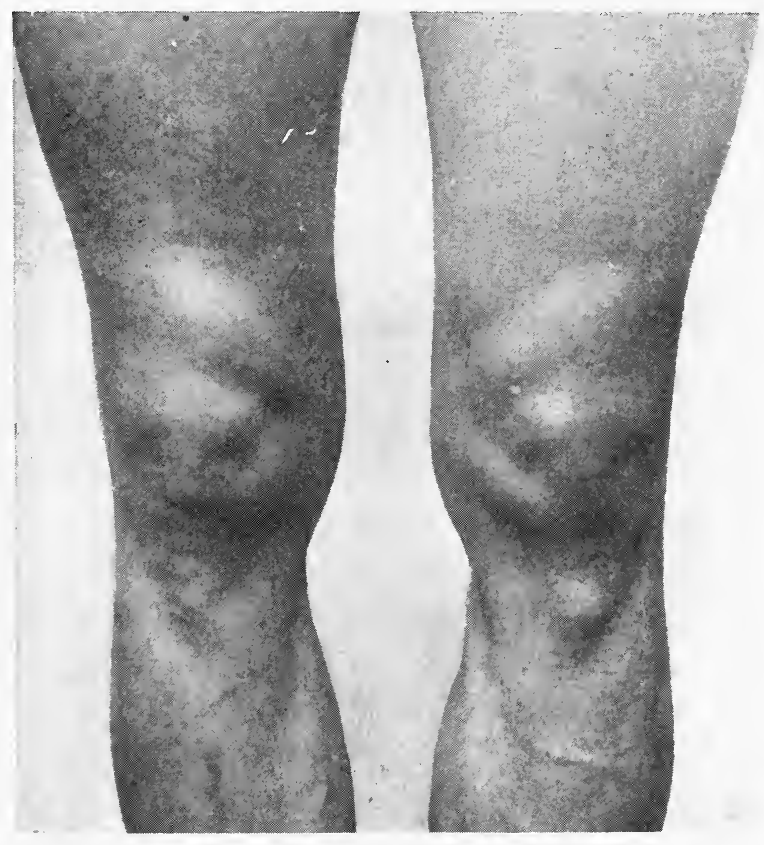

Fig. 46. To illustrate the appearance of a knee-joint, the subject of a chronically thickened synovial membrane. The outlines of the capsule may be clearly noted, while the dimplings of the skin and the fossæ around the joint are not obliterated as they would be in cases of synovitis with effusion. It will be well to compare with Figs. 44 and 45.

admirably the purpose of a 'gentle reminder'; indeed, by some surgeons it is recommended more for the latter purpose. The method advocated for developing and restoring the wasted muscles is treated of in the chapter dealing with massage and movements. 


\section{Sprains IN WhICH THE INTERNaL Lateral}

LIGAMENT IS MOST CONCERNED

The internal lateral ligament is, of all the structures of the knee, that most frequently affected by twisting and wrenching forms of violence. Nor is this surprising, if its situation and attachments are considered. Though a very strong band, and capable of resisting a great amount of strain, it is the main support of the inner side of the joint, so that every stretching force applied to the outer side of the articulation makes its impression upon it. It most generally suffers when the limb being in the extended position is forcibly made to assume or approach the condition of genuvalgum. Any forcible separation of the head of the tibia from the internal condyle puts its fibres upon the stretch, and according as the degree of separation is little or great, stretching or laceration results.

Sprains of the internal ligament are exceedingly common amongst athletes. Football accounts for the greater number; but it is met with in players of every sort of game and pastime. The part of the structure which most readily tears is its attachment to the femur. Next in frequency are the deep fibres which are attached to the capsular ligament and internal semilunar cartilage. The lower or tibial attachment in the writer's experience is rarely torn.

Symptoms and diagnosis. If the sprain has been slight, the patient will probably complain of pain located over the whole of the inner side of the joint, increased by every movement whether of flexion or 
extension. There may be nothing to be seen on inspection. Effusion in such a mild case rarely follows if the joint is a healthy one, but even a slight injury may produce it in one previously damaged.

When, however, the force has been sufficient to produce laceration, the pain is very often considerable at first, lessening gradually later. It is usual to find an intensely painful spot of small size either just over the upper attachment of the ligament to the inner side of the condyle, or exactly opposite the middle of the border of the semilunar cartilage. In most cases it is in the former situation. The swelling is small and localised at the painful spots above mentioned, and is due to hæmorrhage from tearing of a small blood-vessel.

If the case is seen early there may be no effusion into the capsule, but this will invariably come on a few hours later. If there is immediate effusion into the joint, it is due to hæmorrhage, and results from tearing of the capsule and synovial membrane as well as the lateral ligament.

When the pain is localised at the level of the semilunar cartilage, especially when there is swelling from extravasation of blood, the case may be, and often has been, mistaken for a detachment with displacement of the cartilage. To the clinical importance of this fact reference will again be made in the chapter dealing with displacements of the internal semilunar cartilage.

In addition to the pain and swelling, it will be noticed that the limb assumes the semiflexed posture, the patient is unable to extend the joint without considerable pain, or to bear any weight on the limb. 
The history of the injury, direction of the force, a feeling perhaps of something snapping or tearing at the time of accident, taken together with the localised pain and swelling already referred to, and the absence of immediate effusion into the capsule, will go far to establish a diagnosis. Rupture of the deep fibres, or separation of them from the internal semilunar cartilage, followed as it invariably is by localised swelling situated exactly over the line of the cartilage, is recognized by the absence of the other symptoms of slipped-cartilage. When in doubt, the patient should be completely anæsthetized and an attempt made to reduce the suspected displacement. If such an attempt at reduction as is made by the manœuvre described elsewhere (p. 163 et seq.) for the reduction of a displaced cartilage proves a failure, it is to be assumed that there is nothing capable of reduction, and that the swelling represents a blood-clot and not a projecting cartilage. Later in the case, the usual play of colours will demonstrate the locality of the effusion and give a fair estimate of its amount.

The treatment is by elastic pressure and movements, the details of which are described in chapter ii, p. 22 et seq., and in chapter ix.

\section{Sprains in which the External Lateral} LIGAMENTS ARE MOST CONCERNED

The external lateral ligaments are much less frequently injured than the internal. The stretching force necessary has to be applied to the inner side of the joint while it is extended, and, from the very nature of things, as one might expect, is not of 
common occurrence. When the external lateral ligaments yield there is generally considerable tenderness over the popliteus muscle and at the back of the joint, as well as over their lower attachments to the head of the fibula. Association football players may overstrain or rupture their external ligaments, when they cross legs with one another in kicking at the ball from different directions at the same time, each falling with his leg across that of his opponent. Effusion may or may not take place into the synovial cavity. The diagnosis presents no special difficulty, and the prognosis and treatment is the same as for ordinary sprains of the knee.

Separation of the body of an internal semilunar cartilage from the deep fibres of the internal lateral ligament of the joint. It sometimes happens that as the result of a sprain the deep fibres of the internal lateral ligament of the knee become separated from their attachment to the convex border of the internal semilunar cartilage. When this occurs hæmorrhage follows and the blood clotting in this situation gives rise to a definite localised swelling often readily palpable. On several occasions this swelling would seem to have been mistaken for displacement of the cartilage from the joint. Not infrequently when the violence is sufficient to lacerate the synovial membrane as well, bleeding into the joint results. It is quite conceivable that a portion of the blood-clot insinuating itself between the cartilage and the tibia on the one side and between the cartilage and the condyle of the femur on the other, might become organized and produce a definite form of internal 
derangement. Such a condition has actually been noted during an operation in a supposed case of displaced cartilage. Although no definite displacement of the semilunar cartilage was to be found, the organized blood-clot had so insinuated itself between the bones as to render extension of the joint impossible. A sufficient explanation was thus given of the circumstance that every previous attempt at reduction by manipulation had ended in failure.

Sir Wm. Bennett ${ }^{1}$ describes a somewhat similar condition as giving rise to the same train of symptoms, but he attributes the cause to a bruising of the cartilage. It is here maintained that the injury is in the main due to a laceration of the deep fibres of the internal lateral ligament and not always to a mere bruising of the edge of the cartilage.

\section{Schlatter's Disease}

This is the name now given to an injury to the tibia close to the knee. It is met with in athletic boys, between the ages of eleven and sixteen, and results from a sudden and violent strain. In the execution of an athletic performance like broad-jumping, or vaulting, or as the result of a miskick at football, the tubercle of the tibia becomes partially detached with the patellar ligament. The separation of the tubercle of the tibia becomes possible in those cases in which it is developed as a separate centre of ossification; the usual arrangement is that it is

1 Internal Derangements of the Knee-joint. Longmans, Green \& Co., 1903. 
developed in common with the upper epiphysis of the "tibia.

In the process of detachment the ligamentum patellæ takes with it not only this accessory epiphysis but also a varying amount of the neighbouring periosteum. In addition to the detachments of the bone and periosteum there is hæmorrhage. The effused blood, which may be of fair quantity, finds its way under the detached periosteum and fills not only the gap between the fragments, but also the bursa which normally lies between the lower end of the patellar ligament and the head of the tibia.

This lesion must not be confounded, as it sometimes has been, with fracture of the upper epiphysis of the tibia in which its overhanging beak-like projection has been snapped across by direct or other violence.

The following history given in a recent case very aptly describes the usual onset and early symptoms.

'I was broad-jumping at school and in landing felt something snap and was unable to stand. The pain was not severe, and limping home I saw the School Matron, who bathed my knee with hot water and applied a bandage. The next day I could walk but not run. I felt stiff, but as there was little or no pain and the bruising was not much, I did not see the School Doctor.'

The condition can hardly be said to be rare, as during the last year I have seen five cases.

As time goes on the pain which at first is described as trifling becomes worse, and the patient finds that he is unable to run, or to take part in active games. The swelling increases whenever he takes a long 
walk, and becomes so tender as to prevent his kneeling. The slightest local pressure increases the tenderness, whilst the pain, which has now become constant, and of an aching type, is sufficient to keep him awake at night. The knee-joint always becomes stiff during the periods of repose, and painful afterwards when first moved. Having arrived at this stage, perhaps for the first time the surgeon's advice is sought.

On examination, in the early stages, a distinct swelling will be seen and may be felt over the tibial tubercle; this may or may not be tender. Viewed from the front it is rounded; in profile, with the knee bent, it presents a distinct convex projection forwards. There is a certain amount of discomfort when the joint is bent. The swelling is at first due to hæmorrhage. As time goes on it becomes smaller and harder and is due to ossification in the clot. The deformity for this reason remains permanent. Fig. 46 is a photograph showing the characteristic swelling over the head of the tibia and the permanent deformity in the left leg of a man who in boyhood had 'sprained his knee'.

In a few cases a bony plaque or raised thickening continuous with the anterior surface of the tibia remains as the only disfigurement. In others, and especially in those which have been treated continuously, and for long periods, by massage, in addition to the bony thickening around the tubercle, an ossification in the lower end of the patellar ligament itself may be noted.

Fig. 47 is a radiogram of the knee of a schoolboy aged sixteen, who three and a half years before gave 
the joint a sharp wrench while playing football. Nothing was thought of this by the matron, and "he was allowed to go on playing for six weeks, while

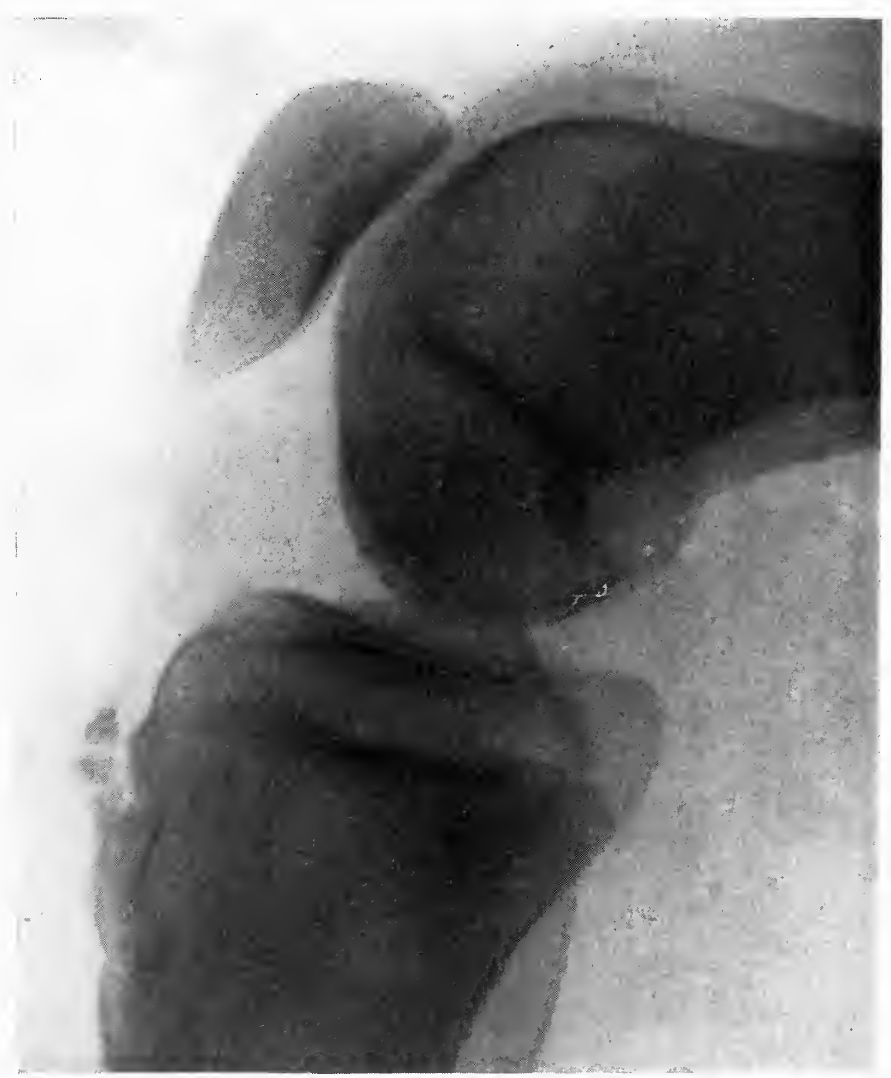

FIG. 47. Radiogram of case of Schlatter's disease. Note the separation of the tibial tubercle and ossification in the lower end of the patellar ligament.

the lump below the knee grew bigger and daily more painful'. After six weeks he saw a medical man, who advised him to wear a knee-cap, stopped his games, and told him at that time that he would be 
all right in ten years. His parents then sought for him other and special advice, and he was taken from one specialist to another. The concurrence of professional opinion was that he would get well in time, but that he must give up all athletic exercises. In the end he was advised to have Swedish massage, which was continued daily for a whole year. When seen three and a half years after the first injury he still had a lump at the head of his tibia, which became tender after a long walk. He also complained of an intermittent stiffness in the knee, worse when he first got out of bed in the morning. He could not take part in any violent games with pleasure, for he 'readily became stiff and his pace in running was gone'. The accompanying radiogram was taken at that time and shows clearly the reasons for his disability. If examined closely there will be seen an old detachment of the epiphysis for the tibial tubercle as well as some detached fragments of bone in the lower extremity of the patellar ligament, just anterior to the situation of the patellar bursa. It may be easily realized how much less supple the patellar ligament must be when thus reinforced by processes of bone growing amongst its ligamentous fibres. Such an irregular and stiffened ligament must press unduly upon and irritate the immediately subjacent bursa. Every attempt at active exercise produced swelling probably by inducing a subacute bursitis. Frictionmassage continued as the treatment for many months was probably the cause of the ossification in the tendon, for we know that in other situations of the body myositis ossificans is apt to be made worse by friction. Fig. 48 is the radiogram of a similar case. 
It was taken from a boy of fifteen, who injured his leg twelve months before in running forwards to vault over a gymnasium horse. He had been treated at

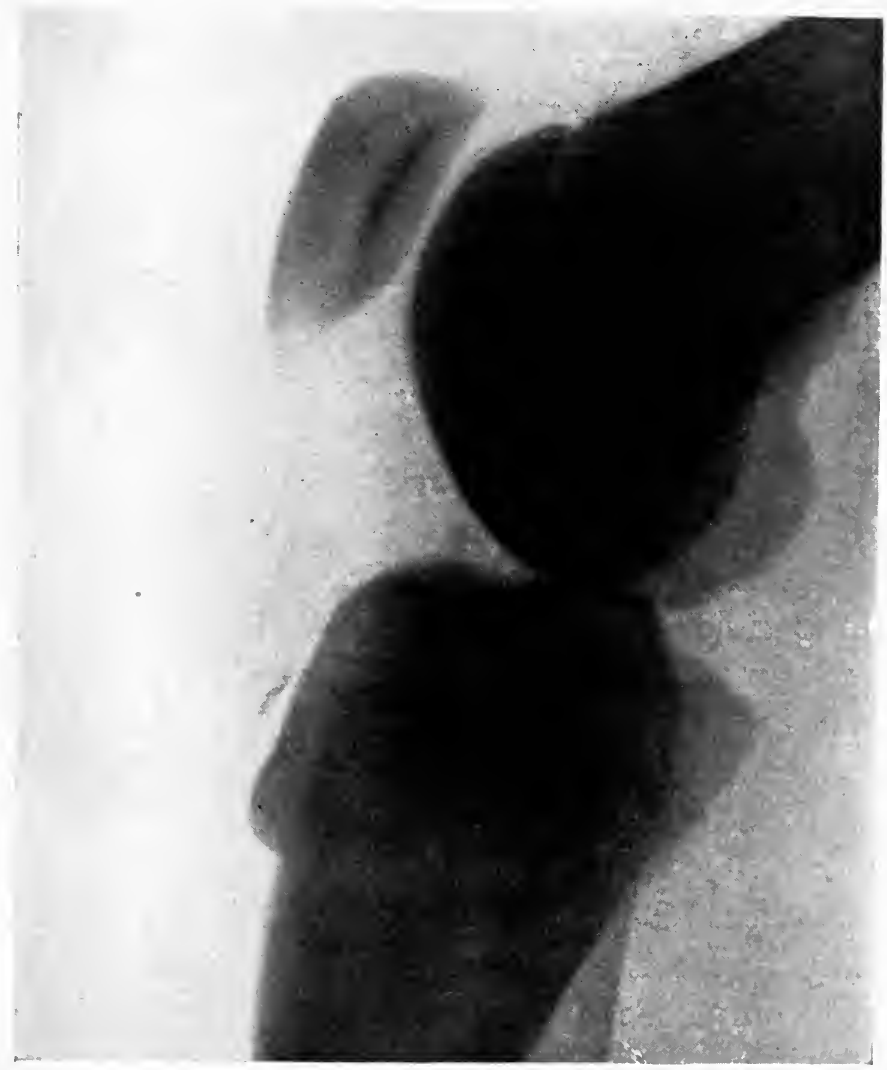

Fig. 48. Case of Schlatter's disease. Note the displacement of the tubercle of the tibia upwards and the small centre of ossification in the patellar ligament.

school and elsewhere by rest, blistering, bandaging, electricity, and continued massage. On examination, he complained of 'being unable to walk far, or to play at athletic games. Whenever he exercised himself 
the swelling at the top of his leg increased, became hot and throbbed. The pain after exercise was worse at night, and the joint was fixed each morning when he awoke; no treatment other than rest seemed to benefit him'.

The picture shows a pulling upwards of the tibial tubercle without complete separation, and in addition, a small centre of ossification beginning in the lower end of the patellar ligament. The figures are interesting in so far as they clearly depict the precise anatomical and pathological conditions of the disability, and they help to explain the reasons for some of the symptoms.

The increase in the swelling after exercise is probably due to a bursitis. It is possible also that friction-massage carried out assiduously, and for a long time, is more likely to do harm than good. Over-stimulation of a blood-clot in close relationship to lacerated periosteum is not unlikely to lead to the deposition of bone. The loss of function is mainly due to stiffness and inability to bend the joint quickly, for there is comparatively little weakness or true loss of power.

The diagnosis must be made from separation of the upper epiphysis of the tibia, from fracture of its tongue-like and downward projecting process, and from a superficial bursitis. The precise history of the injury, the comparatively slight pain and loss of power experienced in Schlatter's Disease, usually serve to distinguish it from the more serious injury; but the use of the X-rays will alone definitely establish the diagnosis. Whilst the immediate prognosis is uncertain, the remote is good. 
All the symptoms except the deformity pass away in the end.

The treatment of ordinary cases is mainly prophylactic. All violent exercises and kneeling must be prohibited, and only gentle walking exercises with a stiff knee permitted. A roller-bandage to allow of equable compression and to give support during the day is comforting; this may be removed at bedtime to allow of counter-irritation for the relief of the bursitis.

Gentle massage to diminish pain and aid absorption is sometimes beneficial early in the case, but if continued as a routine method of treatment for long periods may result in permanent injury.

Ossification in the ligament has been known to follow upon continued massage. In two cases recently it became necessary to perform an open operation. In the one the exigencies of a public service demanded a rapid cure, in the other it was done to relieve severe nocturnal aching. In the former the operation took place two weeks after the accident, in the latter nine months.

After an incision had been made into the swelling in the more recent case, and the blood-clot removed, a partial detachment of the tubercle of the tibia could be seen.

The surrounding periosteum was considerably torn and some of its fibres had passed beneath, and were lying under the detached ledge of bone. The upraised fragment was replaced by a few simple strokes with a mallet, and retained in position by extending the limb and suturing securely the rents in the periosteum and other fibrous tissues. In the old case, instead of 


\section{SPRAINS IN THE REGION OF}

blood-clot the swelling was made up of an irregular, bony, somewhat flattened, subperiosteal overgrowth. From this the thickened periosteum stripped readily. There was no ossification in the patellar ligament. With the hope of relieving the nocturnal pain a chisel was driven through the margins of the new growth into the head of the tibia on each side. The condition found was, however, more of the nature of a chronic periostitis than a true ostitis.

In both cases a rapid cure resulted. The experience gained from these cases, taken in conjunction with the fact that the natural cure is always slow and tedious, lends colour to the view that in suitable cases, and in those where the saving of time is of importance, the open operation may afford the best means of a speedy cure. It is not suggested, however, that an operation should be performed in every case. A few other cases have been recorded during the last two years.

Professor Alexis Thomson ${ }^{1}$ describes an interesting case and illustrates his paper with radiograms. The injury was bilateral. In the same paper attention is drawn to Sir James Paget's Essay on 'Periostitis following Sprains', and the writer quotes extensively from the essay to show the similarity of the description there given with the features of Schlatter's disease as described by that author.

\section{Exostoses as a CAUSE OF KNeE-SPRAIN}

Bony outgrowths (exostoses) in the neighbourhood of the knee-joint are by no means rare. They are usually found in connexion with the epiphysial

1 Edin. Med. Journ., March, 1908. 
lines or growing parts of the bones. The tibia is as liable to them as the femur. The commonest sites are at, or near, the adductor tubercle of the femur, and over the inner surface of the head of the tibia, more rarely, springing from the external condyle of the femur close to the attachment of the external lateral ligament.

During the process of development, and while the bony growths are still small and sessile, the tendons in their immediate proximity are apt to be hitched on to or partially caught by them during the ordinary movements of the knee. Such an accident results in an overstretching of the tendon or ligament with all the accompanying symptoms of sprain. In a recent case in which there was an exostosis growing from the upper epiphysis of the tibia on its inner surface, the tendons of the semi-membranosus, semitendinosus and gracilis muscles were frequently caught either separately or in conjunction, when the knee was suddenly and unexpectedly straightened. Pain, often described as acute and sickening, and lameness are the symptoms usually complained of.

In a few cases a snapping or clicking sound is described.

Tenderness on local pressure is usual. It is caused partly by the overstretching of the fibrous structures, and partly by a traumatic bursitis.

Synovial bursæ usually develop between the tendons and the exostosis over which they play, and from the constant stimulation to which they are liable become enlarged and may lead to considerable swelling. In this variety of sprain there is seldom if ever any accompanying synovitis in the joint 


\section{SPRAINS IN THE REGION OF}

itself. The injury is to be regarded as extraarticular.

When the bony growths become pedunculated they are liable to fracture either by direct violence or muscular action. Fracture of an exostosis frequently leads to spontaneous cure of the symptoms of sprain.

The 'snapping sound' is presumably produced by the sudden slipping of the tendon over the growth, and perhaps, to some extent, by compression of the contents of the interposed bursa. It is often elicited by the alternate movements of flexion and extension of the knee.

The diagnosis is, as a rule, simple when the exostosis is large enough to be palpable. Treatment consists in removal of the growth. No other method is curative. When for any reason an open operation is contra-indicated, local counter irritation affords most relief since it influences the accompanying bursitis. Relapses must be expected when only nonoperative methods are adopted.

\section{Partial Dislocation of the Patella OUTWARDS}

The patella in some individuals has a tendency to displacement outwards. Normally, the line of action or pull of the quadriceps tendon does not correspond with that of the patellar ligament. In certain conditions such as genuvalgum or chronic synovitis of the knee-joint, the axes of these lines of action become even more dissimilar, so that there is a distinct tendency for the patella, placed as it is at 
the angle between the lines of traction, to become drawn outwards during the extension of the knee. Dislocation outwards is normally prevented on the one hand by the resistance offered to the patella by the outer margin of the trochlea surface of the femur, and on the other by the support given to it by the internal lateral expansion of the quadriceps tendon, which attaches it to the capsule and inner side of the limb. If for any reason the outer edge of the trochlear surface is ill-developed, or the ligamentous attachments of the patella at its inner side become weakened by previous injury or disease, the tendency for the patella to pass outwards is increased. With such predispositions as those mentioned, even a moderate degree of violence will be sufficient to allow of partial dislocation of the patella during forcible extension of the knee.

The injury is accompanied by all the usual symptoms of sprain of the knee, such as pain, loss of function, swelling and effusion into the synovial cavity.

Pain is usually referred to the inner side of the joint, and is increased with the movements of flexion and extension. The joint becomes distended with fluid, and the limb weak and insecure.

The diagnosis may be made by observing the movements of the patella during flexion and extension of the knee, when it will be seen to pass further outwards than usual. There is always a risk in neglected cases for the patella to become completely displaced. The treatment in a mild case is the same as for other varieties of sprain. In relapsing and more aggravated cases, something more may be necessary. The patient may be so incapacitated by 


\section{THE REGION OF THE KNEE-JOINT}

the repeated attacks of synovitis that aspiration of the joint or even arthrotomy may be called for.

In every case of arthrotomy the surgeon's aim should be to suture accurately all the torn fibres of the accessory extensor apparatus on the inner side of the knee as well as any rents in the capsule itself, and to use every means in his power to obtain firm union.

The subjects of complete dislocation of the patella outwards are so prone afterwards to these partial dislocations with all the inconvenience appertaining to them, that it becomes a question whether open suturing at the time of the injury may not be on the whole the most satisfactory means of dealing with this troublesome affection. 


\section{CHAPTER VI}

\section{INTERNAL DERANGEMENTS OF THE KNEE-JOINT}

'Displaced Semilunar Cartilage,' 'Subluxation,' 'Slipped Cartilage,' are terms used synonymously for a strain to which the knee is liable. It is usually the result of sudden violence in the form of a wrench or twist whereby the attachments of one or other semilunar cartilage becomes unduly stretched or torn. A cartilage may, however, become so extremely movable from a gradual stretching of its ligaments as to be caught between the bones even during ordinary movements. It is more common in males, and may occur at any period between the ages of twelve and sixty ; before or after these periods it is almost unknown.

When the ligaments are extensively torn, and the movements proportionately free, the cartilages are often dislocated inwards into the joint and come to lie in the space between the femoral condyles. More rarely they are displaced outwards. The writer has never met with a case of typical outward displacement, although such cases are on record.

Not only are the ligaments liable to stretching and laceration, but the cartilage itself may be torn across partially or completely (vide Fig. 49). When the tear is complete, the detached portion is liable to be caught and squeezed between the bones. 
Lacerations have been met with in almost every part of the cartilages, but for the most part they occur towards the anterior extremity ; for not only is this the weakest part anatomically, but it is also the portion most liable to strain.

From an examination of many strains, and from his operative experiences, the writer has formed the

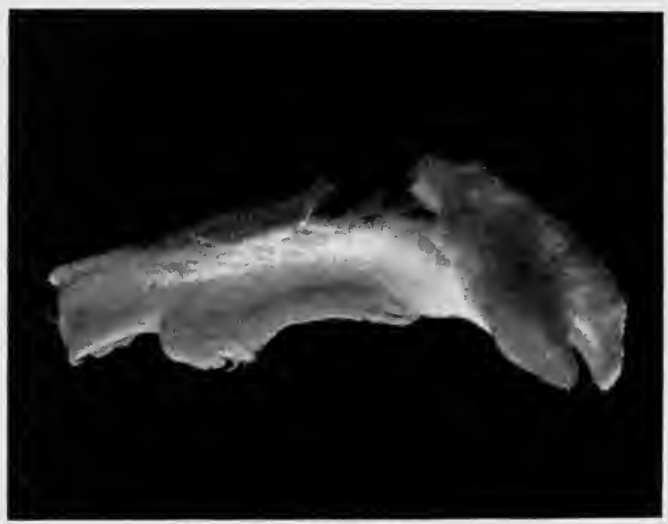

FIG. 49. The anterior half of an internal semilunar cartilage of the knee removed by operation for partial detachment and chronic synovitis. The larger end is posterior; the specimen was cut from the posterior fixed portion. Note the thinning out and internal projection of the inner margin of the cartilage. This thin margin when caught between the bones, which was frequent, accounted for the occasional sudden pain and the synovitis. The coronary ligaments had been torn, but the anterior horn remained attached in the intercondyloid space.

opinion that the most common site for the cleavage to take place is at a point just in front of the junction of the anterior and middle thirds of the internal cartilage, and not at the anterior extremity, as is often stated. The anterior third is the most movable, as well as the most attenuated part of the structure, and being stretched during every movement of 
flexion and rotation, readily becomes loose. When actual loosening once takes place, every movement of flexion and rotation tends still further to stretch or undo the coronary attachments; eventually, by some sudden or excessive violence, the cartilage

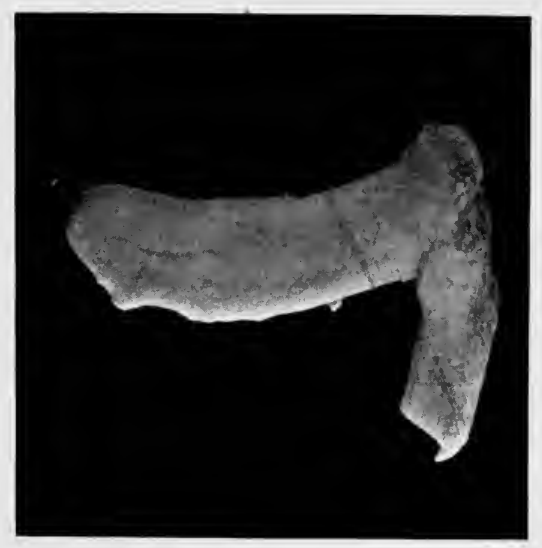

Fig. 50. Photograph of the anterior half of a left internal semilunar cartilage removed by operation. The horizontal portion represents the anterior third of the structure which had become detached from the coronary ligaments at its periphery, but remained attached at its anterior cornual site. The vertical limb represents the posterior part of the cartilage, while the angle between them demonstrates admirably the author's observation that the usual site for laceration of a cartilage is near this point, viz. the junction between the anterior and middle thirds, and not, as generally supposed, at the anterior cornual attachment. The patient, who formerly was unable to take any exercise, is now able to do anything and to play at any violent game, for the detached portion, which was wont to fall between the bones during rotation, has been removed.

already loosened at its anterior border slips backwards into the intercondyloid space and escapes for a time, or it may then and there be completely torn across.

Fig. 50 shows the anterior half of an internal semilunar cartilage removed recently. It had not 
been ruptured, but was loosened from the tibia in its anterior third owing to tearing of the coronary ligaments.

This specimen was obtained from the knee of an undergraduate, aged twenty, who, in October 1907, for the first time, while 'scrumming' at football, slipped his cartilage. Between that date and the time of operation in March 1908 there had been four distinct recurrences; indeed, no sooner had the symptoms disappeared under treatment and the patient begun to walk about, than some trivial movement, such as getting in and out of his bath, again disabled him. Each relapse seemed more easily produced than the former one, while the 'cure' took longer each time.

Growing weary of the inconvenience he decided to have the cartilage removed.

An examination of the specimen shows distinctly the acute angle formed between the anterior movable part and the posterior fixed portion. The longer limb in the picture represents the anterior, the shorter the posterior, where it was cut from the hinder part of the cartilage during its removal.

The anterior limb was directed inwards from its outer attachment towards the intercondyloid notch, in which situation it was seen to lie whilst the limb remained in the extended position. While the joint remained open, so that observations could be made, and the knee was flexed and the leg rotated outwards, this movable portion shifted its position and was brought to lie between the femur and tibia, and so caught that extension was rendered impossible.

During the year 1908 four cases were met with 
while operating in which rupture, partial or complete, had taken place at the spot above indicated. At different times cases have been seen illustrating the successive stages from the first loosening of the cartilage at its anterior coronary attachments to its complete displacement between the condyles of the femur, and so on to that of partial and even complete fracture with displacement. Fig. 50 shows a cartilage with partial detachment at the angle of junction between the anterior and middle portions. In this particular instance a rare opportunity was afforded of studying the mechanism of a kneejoint in which the anterior portion of an internal semilunar cartilage was not only movable, but became displaced and caught, in certain movements of the joint. When ordinary flexion and extension were executed slowly and without any, or with very little, passive rotation by the operator (the patient being under an anæsthetic), the cartilage remained displaced into the intercondyloid notch and was not caught between the bones; but whenever the movements were rapidly executed and considerable rotation allowed, it became nipped between the femur and tibia, so that the joint was locked and complete extension made impossible. The inner margin of the specimen shown in Fig. 49 shows a thinning out from its being frequently nipped between the femoral condyle and the tibia.

On rare occasions the cartilage is split longitudinally instead of across. The writer has met with a case in which an internal cartilage was partially torn into three strips in its anterior two-thirds, so that the two inner portions were flattened out and 


\section{INTERNAL DERANGEMENTS OF}

loose. The most internal strip had been completely detached posteriorly, and passing inwards between the bones was doubtless the cause of the symptoms. The cartilage itself remained intact at its posterior third, and was firmly fixed to the tibia both by its posterior cornu and coronary ligaments. Fig. 51

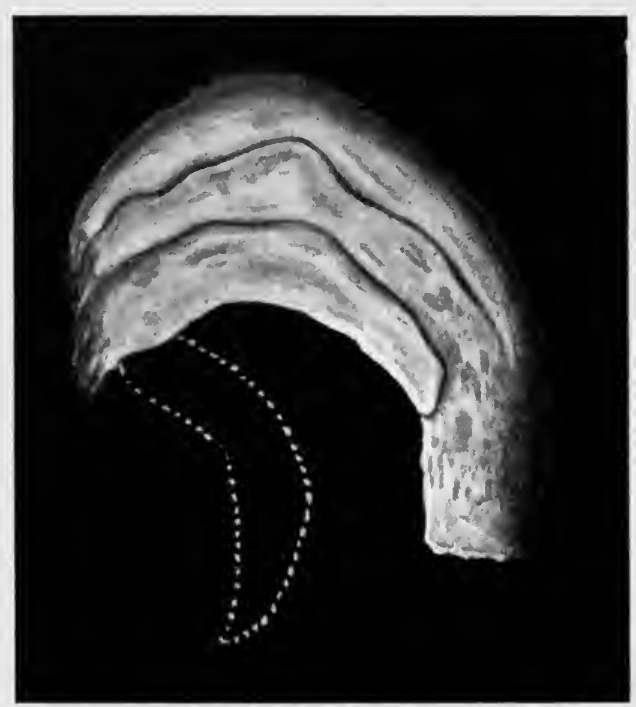

FIG. 51. Drawing from a specimen of an internal semilunar cartilage of the knee, showing longitudinal splitting into three strips in its anterior half. The narrow part is posterior. The cartilage was much broader than normal, and while attached at its usual site anteriorly the innermost strip became detached posteriorly and, as indicated by the dotted line, was wont to slip inwards into the joint and get caught between the bones.

is meant to convey roughly some idea of the appearance of the specimen, which unfortunately was torn by a bystander before it had been photographed.

The patient was a man of thirty-five who, from the age of twelve, had worked in a coal mine. He stated that when he was about fifteen he strained his knee and was laid up for nearly a year off and on, but that he afterwards became quite 
well and able to do his work, although often stiff when he first began work each morning. The joint at times would swell considerably, and, as far as he could remember, he was never able to straighten it completely. This did not inconvenience him in the mine, for, as he said, he was generally lying on his side, or squatting, while at work. It was only when he went for a long walk on Sundays that the joint would swell and 'bother'. There was always some 'clicking' or 'creaking' when the joint was worked ; this both he and the colliery doctor attributed to rheumatism. Shortly before he sought advice he one day ran after a dog, and, in trying to kick it while standing on his weak leg nursing his knee, he wrenched the joint and 'felt something go' with a 'snap', became powerless from pain, felt sick, and fell to the ground. There was some evidence of old osteo-arthritic changes in the joint. The rarity of these cases of longitudinal splitting, together with the interesting history given by the patient, were considered sufficient to warrant a detailed account.

The anterior two-thirds of the cartilage was remcved by the method of operating to be described later, the patient made a complete recovery, and resumed work at a colliery eight weeks after. He has since gone on well, suffering in no way from the osteo-arthritic changes which had already begun at the time of the operation.

The internal cartilage is much more frequently injured than the external. Various factors are concerned in this. First and foremost it may be stated that the internal cartilage does not enjoy the same range of movement as its fellow, while at the same time a greater strain is put upon it during the ordinary movements of the joint.

The external semilunar cartilage is more circular in shape, forms the arc of a smaller circle, while its cornual attachments, both before and behind, are 


\section{4 INTERNAL DERANGEMENTS OF}

embraced by those of the internal. This gives it a greater degree of mobility whereby it escapes to a greater extent the grinding strain of the condyle upon the tibia during the movements of rotation.

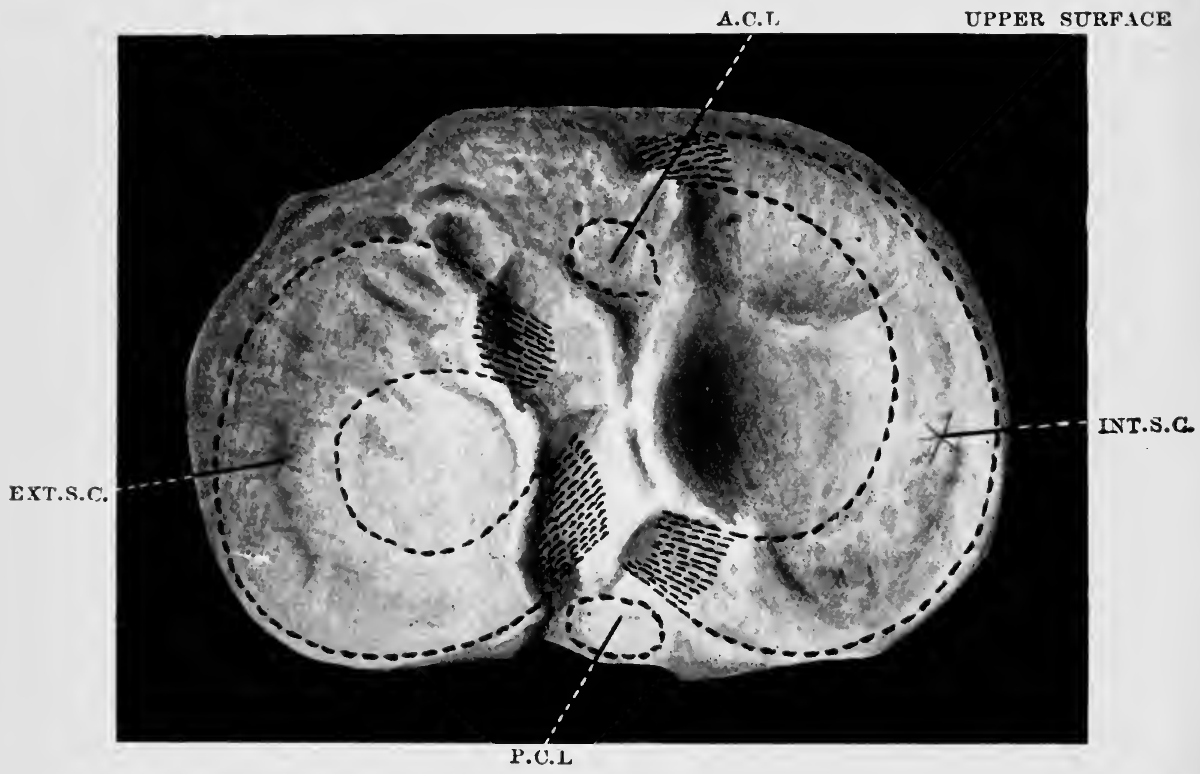

Fia. 52. Drawing made from the upper surface of the head of a left tibia, to indicate by the dotted lines the anatomical relationships of the semilunar cartilages one to the other, and to demonstrate their precise points of attachment, as well as the relative positions of the attachments for the anterior and posterior crucial ligaments.
A.C.L. = Anterior crucial ligament.
P.C.L. = Posterior crucial ligament.
EXT.S.C. = External semilunar cartilage.
Ixт S.C. = Internal semilunar cartilage.

The range of movements of the internal cartilage is furthermore limited by its attachment to the capsular and internal lateral ligaments. The external cartilage is not attached to the long external lateral ligament, and although its border is grooved by the 
popliteus tendon, it does not seem to derive much support from it. Again, if we compare the anatomy of the cartilages themselves, the reason is at once forthcoming.

The semilunar cartilages may be regarded as the remains of what were originally two complete disks interposed between the surfaces of the femur and tibia. By the process of time these disks have been so worn away in certain localities as to become much thinned and assume a semilunar shape. The wearingaway process has progressed to a greater extent towards the interior of the joint, so that the cartilages, originally of equal thickness throughout, now come to possess a thin edge directed towards the tibial spines, and a thicker border or base towards the periphery.

As the strain in all the ordinary movements of the joint is greater on the inner than the outer side, it follows that the internal cartilage becomes more worn away than its fellow, and for the same reason the anterior segment of the internal cartilage is more attenuated and narrower from side to side, than any other portion of either cartilage.

The fact also that the anterior portion of the internal cartilage is the most attenuated explains not only its inferior strength as compared with the other parts of those structures, but materially influences its mode of attachment anteriorly.

The horns of both cartilages are attached to the tibia anteriorly and posteriorly, and it is necessary that attention should be drawn to the precise modes of attachment.

The anterior horn of the internal cartilage is 
attached in front of the internal tibial spine and immediately in front of the anterior crucial ligament, whilst its other end is fixed to the tibia in front of the posterior crucial ligament.

The anterior horn of the external cartilage finds attachment in front of the external tibial spine and behind the anterior crucial ligament, whilst its posterior cornu is bound down to the rough space between the tibial spines in front and to the outer side of the attachment of the internal cartilage. It will be seen that the posterior horns of both cartilages are firmly attached to the tibia, while the external cartilage receives in addition a strong fasciculus from the posterior crucial ligament, as well as an occasional slip $(\text { Poirier })^{1}$ which passes from its outer and posterior surface to be attached to the outer surface of the internal femoral condyle.

The anterior horn of the external cartilage is firmly attached to the tibia, and is a structure of considerable strength, while the anterior horn of the internal cartilage varies greatly as to its strength and precise mode of attachment.

It not infrequently happens that the anterior horn of the internal cartilage is very attenuated and may be attached:

(1) to the periosteum in front of the upper tibial border ;

(2) to the anterior border of its neighbouring semilunar cartilage, taking, as it were, the place of the transverse ligament;

(3) to its usual site by very feeble ligamentous union.

1 Poirier, Traité d' Anatomie Humaine, Tome premier, p. 674. 
When such an enfeebled development of the cartilage exists there is a strong predisposition to its displacement, and this fully explains why the internal is so much more frequently displaced than the external cartilage. The main body of each cartilage is fixed to the upper margin of the tibia and held firmly in position by the coronary ligaments. The internal cartilage also derives firm attachment at its inner border or base, especially at its middle portion, from fibres both from the capsular ligament and the deep fibres of the internal lateral ligament of the joint, while the external receives no corresponding reinforcement.

The reinforcing fibres from the deep portion, or as it is sometimes described, the deep internal lateral ligament, are, as we said previously, and as we shall see again later when treating of a special sprain of the knee in which they are mainly concerned, of very great surgical and clinical importance.

In certain cases the body of the cartilage becomes separated from its coronary and capsular attachments, although the cornual attachments remain intact.

'The only common form of displacement, however, is that in which the anterior part of the internal cartilage is torn away from its tibial attachment, or from the transverse ligament, occupying any position between the two extremes of outward projection towards the triangular space that lies on the inner side of the joint between the patella, femur, and tibia, and that of inward dislocation into the intercondyloid notch. Most commonly, the floating end of the cartilage occupies a position intermediate between 
those two extremes and is consequently liable to be nipped between the two bones at any moment.' ${ }^{1}$ The free end is liable to be caught and even ruptured during the ordinary movements.

The following changes have been observed as taking place in the detached portion; sometimes it becomes flattened out and fibrous, or contains calcareous deposit, at others bulbous and thickened, but in most instances, and except in those of very long standing, it retains to a remarkable degree its elasticity and truly cartilaginous appearance.

When detached, these portions may even increase in size and present a smooth polished surface.

The determining cause of a displacement is usually some sudden twist or screw action of the body while the leg remains firmly planted on the ground. It can only occur when the limb is to some degree flexed. When the femur is forcibly rotated outwards on the tibia the internal condyle is brought into intimate contact with the anterior extremity of the cartilage and puts it on the stretch. If the stretching force is great or sudden it is quite easy to understand how the cartilage may be torn at its weaker anterior attachment, and in this way become displaced. Various forms of athletic exercises predispose to the injury: football, skating, lawn-tennis, cricket, and other games afford numerous opportunities. 'Scrumming' at Rugby football, whereby the feet are firmly planted on the ground and, while the knees are semiflexed, the body is made to swing forcibly to one or other side, is eminently suitable. Figure-skating,

${ }^{1}$ L. B. Rawling, 'Displacement of the Internal Semilunar Cartilage of the Knee-joint.' Practitioner, September, 1907, p. 358. 
where the performer swings quickly round and pushes off with the foot, is often a cause. It occurs more frequently in the left knee than in the right, as most sudden movements are made from right to left, so that greater strain is put upon the left knee. ${ }^{1}$

\section{Symptoms and Clinical Characteristics}

The advice of the surgeon may be sought either at the occurrence of the first disaster or later, after many recurrences.

The history is usually a very definite one. The patient, while playing at some game, or as the result of a sudden and violent movement, is seized with pain, often so intense as to cause him to fall to the ground in a fainting condition. There may have been a sensation as of something having given way, and a distinct click or crack may have been heard, and if the cartilage remains nipped between the bones, the limb may become 'locked' in a slightly flexed position. So long as the displacement remains, the position is always one of slight flexion ; complete extension is impossible and painful, while full flexion is usually comparatively easy and almost painless. If pressure is made over a point corresponding to the anterior attachment of the cartilage, i.e. in the line between the bones close to the ligamentum patellæ, acute tenderness will be complained of.

Sometimes spontaneously, at other times by the assistance of a bystander, the cartilage slips from its position between the bones, returns to its natural

1 Sir William Bennet, K.C.V.O., Internal Derangements of the Knee-joint (Longmans, Green \& Co.), p. 74. 
position, full extension is permitted, and the patien may even be able to walk, though lamely. Palpation, as a rule, is of very little assistance in making a diagnosis, for owing to the tenderness which is usually present, the patient objects to its practice ; and as the cartilage, in the very great majority of cases, is displaced inwards towards the joint, it cannot be felt. Considerable effusion rapidly intervenes, and in the space of a few hours the capsular ligament may be distended to its fullest capacity, and the patient thereby confined to his sofa perhaps. for weeks. In course of time, especially if treated by rest, massage, and early movements, he may make a complete recovery. As a rule, however, when the displacement results from much tearing of the ligaments of the cartilage, or when the cartilage itself has been torn across, recurrences may be expected. These repeated attacks are liable to occur at any time, causing much inconvenience, and in the worst cases may even lead to disorganization of the articulation and permanent disablement.

In the recurrent displacements the symptoms are of a similar nature, though they may not always be of equal severity. The patient usually learns some dodge or particular manœuvre for reducing the displacement. Sooner or later the joint becomes weaker; it slips out at the most inopportune moments, so that the subject may neither be able to enjoy life nor earn his livelihood. He then seeks the advice of a surgeon, or perhaps a bone-setter, as to the means whereby the displacement may be prevented and the joint restored to its former strength.

There is a prevalent idea amongst surgeons, and 
not apparently without reason, that osteo-arthritic changes are liable to follow on these repeated attacks of acute recurring synovitis.

The prognosis of recurrent displacements is therefore far from good, and steps should be taken in every case to prevent them either by palliative or curative means.

Diagnosis. The differential diagnosis has to be made from

(1) sprains of the lateral ligaments of the knee ;

(2) loose bodies in the joint, whether free and unattached or pedunculated and attached;

(3) thickened outgrowths and hypertrophied fringes of the synovial membrane;

(4) partial separation of the internal cartilage from the deep portion of the internal lateral ligament of the joint.

An inquiry into the history of the case, and the exact site of greatest tenderness, will often be of assistance in differentiating between these varied conditions.

In the case of a displaced cartilage the pain is always referred to the same spot, while in other loose bodies it may be at any part of the joint, and varies in intensity from time to time. Thickened synovial fringes and outgrowths, when they become caught between the bones, so closely similate a movable cartilage that a correct diagnosis is impossible without opening and exploring the joint. Detachment of the internal lateral ligament from the cartilage gives rise to a painful swelling situated immediately over the middle of the basal border of the cartilage, coming on soon after the occurrence of the injury, WHITELOCKE 
and due to hæmorrhage. Palpation will elicit a boggy swelling, tender to the touch, liable to increase from subsequent inflammatory changes, and followed some days later by the usual signs of bruising-play of colours. The characteristic locking may be absent, though complete extension will be impracticable.

Treatment of the first attack is for convenience divided into two stages:-

1. The immediate treatment, or reduction of the displaced cartilage.

2. The subsequent treatment.

Various methods and devices have been adopted for the reduction of a slipped cartilage, some employed by the profession, others by the patients themselves.

Amongst them may be briefly noticed that of alternately flexing and extending the joint, combining with these the movements of rotation. Though often successful, this is by no means a reliable plan, and is the method generally adopted by bystanders and others.

Another method, which involves practically the same principles as the last, is that to which some years ago attention was drawn by a pamphlet, ${ }^{1}$ as having been first suggested by a master at one of our noted public schools. It consists in making the patient, when it is possible, engage in swimming. The alternate flexion and extension, together with the amount of rotation involved in the act of swimming, is all that is necessary to effect reduction. It is a simple and generally effective device, but as it is not often that a swimming-bath is at hand, and

${ }^{1}$ Football Injuries, by R. H. Anglin Whitelocke, M.D., F.R.C.S. (J. \& A. Churchill), 1904. 
as many persons are unable to swim, it is more convenient to place the patient prone upon the floor and instruct him to imitate the movements of swimming. It has been a matter of surprise how very generally successful the practice is. The patient, often amused and not a little curious, strikes out vigorously, takes his muscles unawares, as it were, and succeeds at the very first attempt in completely extending the joint.

It has been considered useful to refer in this place to such a plan, for these accidents occur at such unexpected times and in such inconvenient places that skilled assistance may not be forthcoming. A patient, the subject of recurrent attacks, is thus enabled to help himself early in the case, and gain much time in starting the subsequent treatment.

The method which for some years has been invariably practised, and which is based upon anatomical considerations, is here illustrated, for the sake of clearness, by photography (Figs. 53, 54, 55, 56). It is a plan which is not only safe but convenient, is suitable for longstanding displacements as well as recent, and when properly carried out seldom fails.

It consists in making the patient, his clothing having been sufficiently removed, lie flat upon the floor or some low couch or bed. The surgeon then, standing on his outer side, flexes the leg upon the thigh and the thigh upon the body as much as is possible. The flexed knee is then adducted so as to cross the middle line of the body and come to lie across the navel. In this position the hamstring muscles as well as the extensors of the thigh are fully relaxed, while the internal and external lateral liga- 


\section{INTERNAL DERANGEMENTS OF}

ments of the knee are in their slackest state. The surgeon then grasps with the one hand the lower end of the femur just above the knee, and with the other the region of the ankle, and while keeping the patient's thigh in the fully flexed position with the aid of his own knee, he abducts the leg from the thigh

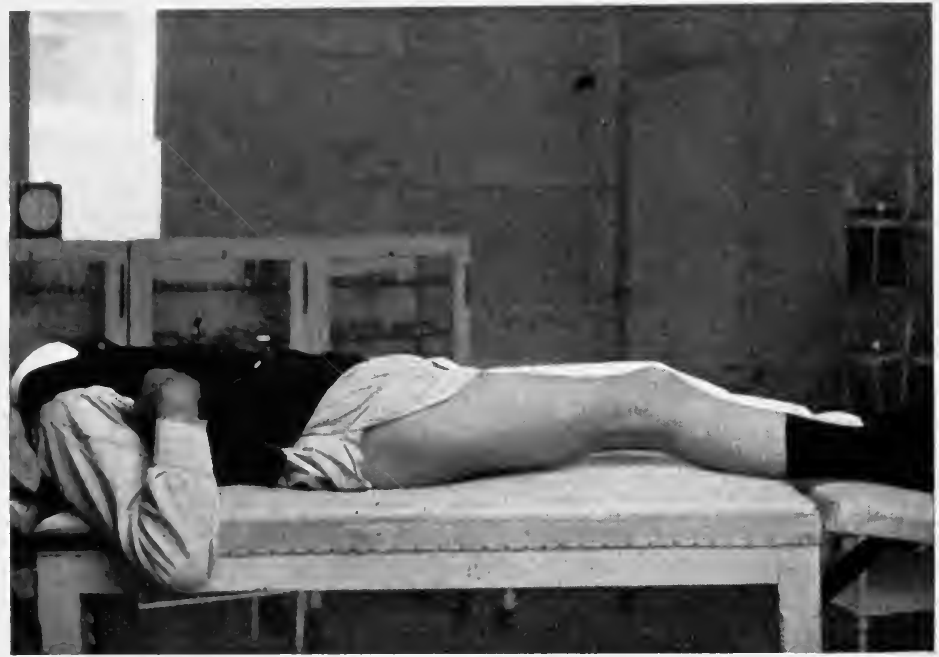

Figs. 53, 54, 55, 56, illustrate the author's method of reducing displacements of the semilunar cartilages of the knee.

FIG. 53. The posture assumed by a knee in which the cartilage has not been reduced and is still out. Note signs of atrophy in the limb.

so that the greatest possible interval is allowed between the internal condyle and inner tuberosity of the tibia. In many instances when this stage has been reached the surgeon's hand grasping the knee may experience a sensation as if something slipped beneath it, or he may hear a click, a snap, or other noise indicative of the movements of the cartilage. In most cases this looked-for result does not arrive 
until after the next stage, that of rotating the tibia upon the femur first internally and then externally, followed by a smart and sudden extension of the limb. When the reduction has been effected the limb at once becomes capable of complete extension and the patient experiences immediate relief.

The surgeon must not be satisfied until the extension is as complete in the injured knee as in the

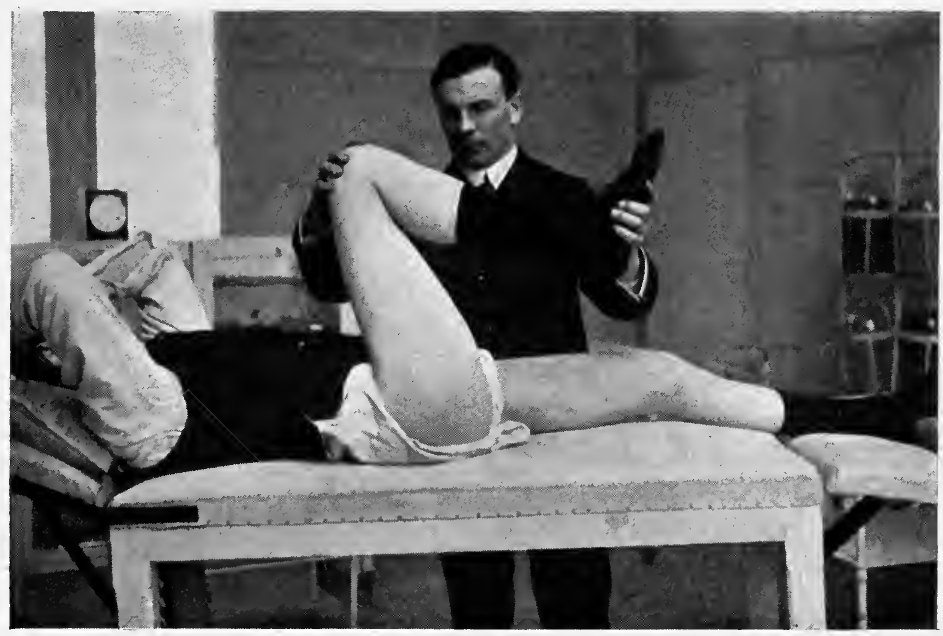

FIG. 54. First position with flexion of knee- and hip-joints.

other. In recent cases of displaced cartilage an anæsthetic is rarely needed, as the manœuvre is not painful and patients usually readily submit to it. In women, for other and obvious considerations, it may be better to administer an anæsthetic.

In longstanding cases, where the inability has existed for weeks and even months, and where subsequent inflammatory changes have, taken place or are suspected, an anæsthetic, besides rendering the 
manipulation simpler, will enable the surgeon to make a more complete examination and satisfactory differential diagnosis. If it happens that one or two attempts fail, in a recent case, an anæsthetic should be administered, and an attempt made to decide once and for all whether it is a case of 'slipped cartilage' proper, or one of hypertrophied syno-

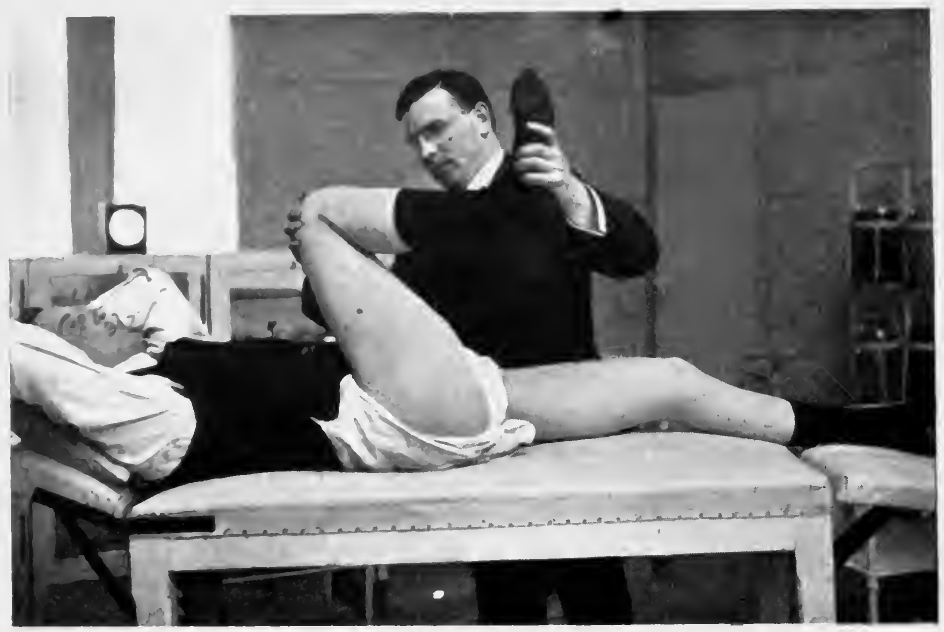

FIG. 5\%. Second position of complete flexion with adduction across line of the body for replacing an internal semilunar cartilage, vide text. Compare the directions of the foot in Figs. 55 and $\Sigma 6$.

vial fringes or other condition giving rise to a somewhat similar train of symptoms.

When reduction by manipulation is impossible, even with the aid of an anæsthetic, it will be necessary to open the joint and remove the cause. The details of such an operation will be considered later.

2. Subsequent treatment. As soon as the cartilage is replaced means should be adopted to check 
as much as possible the outpouring of serous effusion into the capsular cavity. The success or otherwise in preventing or limiting the effusion will depend upon the length of time that has elapsed before the patient comes under proper surgical treatment, and to the amount of perfunctory assistance or violence rendered by bystanders or officious friends. These

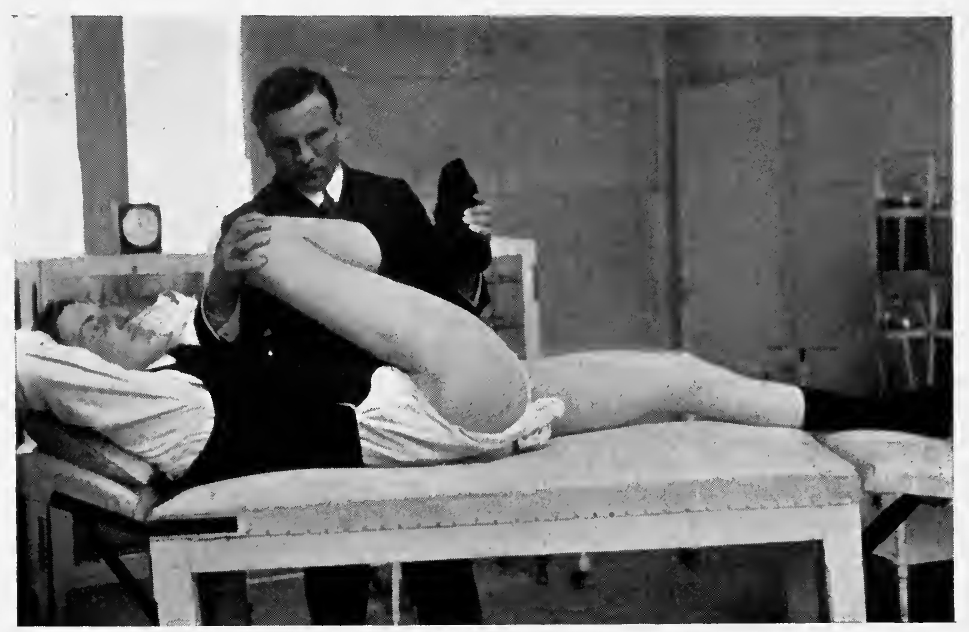

Fig. 56. The position for replacing an external semilunar cartilage. For the sake of clearness the operator is here depicted as standing on the inner instead of the outer side of his patient.

persons, with the best intentions in the world, often contribute unconsciously to the seriousness of the case. When seen soon after reduction, the patient should be put to bed or confined to a couch. To check effusion and to facilitate its absorption, nothing is of greater service, or indeed as useful, as the method already described as applicable to all sprains with effusion, namely, elastic pressure with the aid of a pad of cotton-wool and tight bandage. 
This application enables the knee to assume the posture of greatest repose-that of semiflexionwhereby the pain is diminished. It is sufficiently of

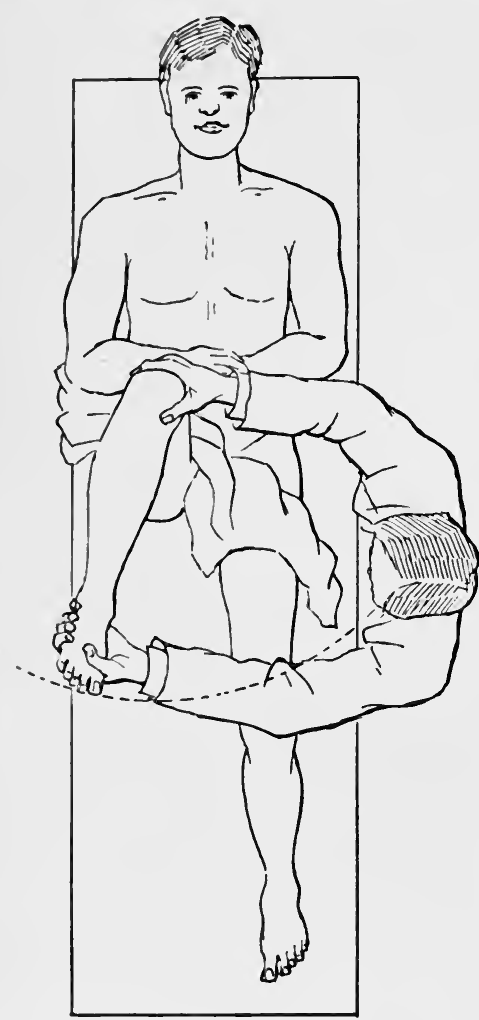

A

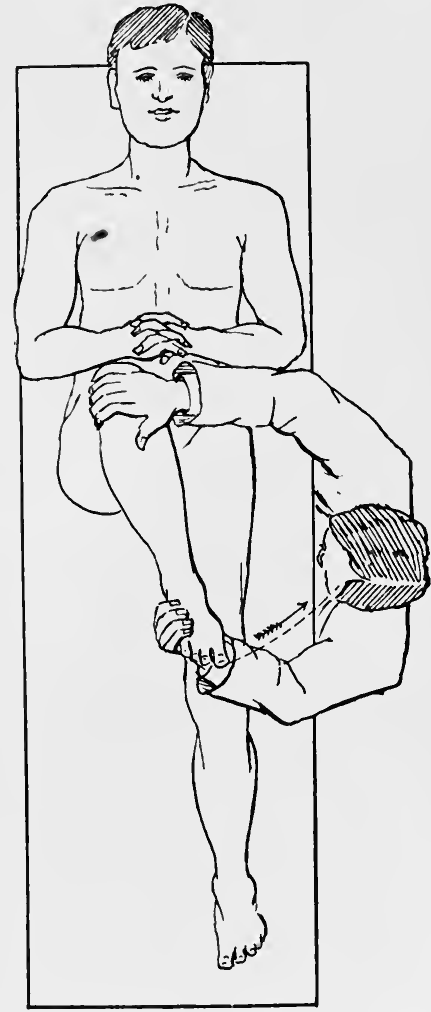

B

Fig. 57. A and B are schemes to illustrate further the author's method. The arrowheads indicate the directions in which the leg is moved or swung in disengaging a locked cartilage. In scheme $\mathbf{B}$ the operator's right hand should be placed on the lower thigh instead of on the head of the tibia.

the nature of a splint to secure temporary rest, and can be easily and conveniently removed and reapplied once or oftener each day during the first few days. 
Treatment by ice-bags and warm applications only delays matters, and does not favourably influence absorption. Nor should any fixed apparatus like a plaster splint be used. Such a knee should be treated in the same way as any other sprained joint, and the possibility of stiffness arising from the formation of adhesions should always be guarded against.

The effusion should be absorbed in about a week, and until this has all but disappeared neither massage nor passive movements are of service: on the other hand, active movements of the limb remote from the joint, such as those of the ankle and foot, should be encouraged from the very first. These active movements of the muscles of the leg and foot, while putting no strain on the knee itself, influence its nutrition, and prevent anything like muscular atrophy in the muscles acting on the knee. Passive movements may assist in preventing or in stretching newly-formed adhesions in some joints, but in the case of the knee they also must tend to move the cartilages themselves and drag upon their newlyformed attachments, the very thing that we wish to avoid in this particular instance. Passive movements cannot prevent atrophy; they act simply mechanically, never physiologically, and may well be entirely dispensed with in this affection.

Massage in the early stages is not only unnecessary, but is inferior in its utility as compared with elastic pressure, where rapidity of absorption is the keynote of treatment. Its uses, however, in the later stages of sprain treatment cannot well be overrated, and the author is second to none in its advocacy when: old blood clot and solid œedema require 
removal. After the effusion has disappeared active movements should be daily increased, providing no strain of a rotatory kind is permitted; a firm domette or crêpe-velpeau bandage should be substituted for the wadding, and the patient allowed to get up, but not to walk. The knee should at first be always supported with a firm bandage so applied as to keep the joint still. This may be removed once or twice daily to allow the joint to be massaged, or the whole limb may be rubbed without removing the kneebandage. An all-rubber bandage should in no case be applied. Such an appliance is too powerful. It not only prevents the proper supply of oxygenated blood from being brought to the joint, but it is very often uncomfortable.

In about a fortnight's time the patient may be permitted to walk, still wearing a bandage. $\mathrm{He}$ should be instructed to avoid any movements of rotation, and to keep the foot well turned out. The bandage need not be worn at night after three weeks, but should be re-applied before getting out of bed each day, and worn continually during the day for at least a month or five weeks.

The bandage will in no way prevent displacement, but acts as a reminder that liberties may not yet be taken, nor violent exercises resumed.

In a certain small proportion of cases treated by rest, massage, and movements, a complete cure results, but the vast majority become victims to recurrence.

Many surgeons advocate a prolonged system of treatment by plaster splints and other fixed apparatus, and while from theoretical considerations this 
would seem only reasonable and common sense, the writer has been led to believe, after a considerable experience, that the cases treated by the shorter régime and with movements from the first, are, to say the least, no more liable to recurrence. For some reason the re-attachments of a loosened cartilage seldom become firm enough to keep it in place, and it is possible that a considerable number of cases of ' cure' have really not been in the first instance true cases of displaced cartilage. The experience of most operators as to the unsatisfactory results of suturing down the cartilages supports this contention. From an examination of many specimens of ruptured or even partially torn cartilages removed by operation, the writer has seldom if ever met with anything giving the definite appearance of an attempt at union. Sometimes one finds the floating end of a detached cartilage bulbous, rounded off, even smoothly polished. The same observation applies to portions of articular cartilages that have become detached by accident. A careful examination will usually show that the once roughened edge or surface of such a detached portion is becoming rounded off and covered over with hyaline cartilage. Fig. 58 was taken of a specimen from the knee of a gentleman who, two years previously, had been bumped against the edge of a gate by a restive horse while out hunting. He had suffered considerable pain, and had been laid up for some weeks and treated elsewhere. He sought advice because occasionally he experienced sudden pain when mounting his horse, followed by swelling of the joint, never at any other time. This became a nuisance, and he was advised 


\section{INTERNAL DERANGEMENTS OF}

to have his knee explored. When the joint was opened the semilunar cartilages, ligamenta alaria and mucosa were all intact and natural to look at, but the synovial membrane had the appearance of yellow ochre, and some yellow semi-gelatinous material could be expressed from the capsule.

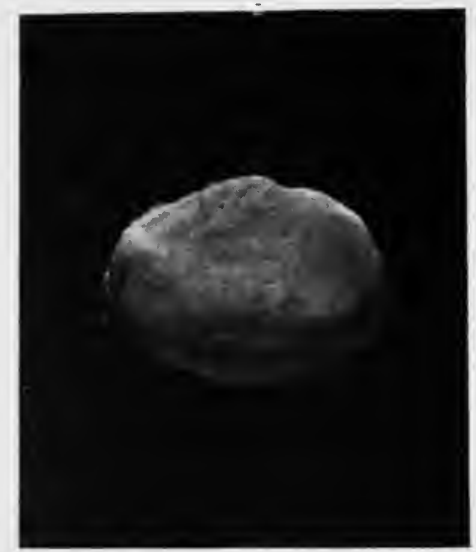

Fic. :8. A portion of bone detached from the edge of a patella some years previously. The specimen had become almost entirely covered by hyaline cartilage, which had grown over its fractured edges from the periphery. It is illustrative of the formation of one variety of loose bodies in a joint, and of the growth of cartilage over detached fragments of bone in joints. About twice its natural size.

From the under surface of the outer condyle of the femur was projecting the specimen here figured. It fitted into a depression in the corresponding surface of the tibia. On removal it was found to be a piece of bone almost completely enclosed in hyaline cartilage. On the outer edge of the patella was a roughened patch also completely covered with hyaline cartilage from which this piece had evidently been detached two years before. The hyaline cartilage 
had grown over both the roughened surfaces of the patella and that of its detached fragment, and well illustrates the process of formation of at least one variety of loose cartilage in the knee. As to whether this growth of cartilage over the bones is mainly due to the effect of polishing movements, or to some protective influence exerted by synovial secretions upon detached bodies, it is difficult to give an opinion ; still, the observation is interesting in this relationship.

Treatment of recurrent or relapsing displacement of an internal semilunar cartilage. As was stated before, the majority of cases suffer a relapse, and the resulting disability becomes one of serious inconvenience, to say nothing of the risks of permanent disablement to which such a joint becomes liable, either from its disorganization and ultimate weakening, or from crippling osteo-arthritis. The surgeon has often to decide which is the more suitable line of treatment. If we assume for the moment that the patient has experienced at least one definite recurrence, then two lines of treatment are open to us-the one tentative or palliative, the other curative or permanent.

1. The palliative treatment is by the utilization of some such apparatus as the one described in Fig. 59, p. 174. The essentials of such an appliance are that while it allows freely of flexion and extension of the joint, it prevents or controls rotation of the tibia on the femur. Various instruments have been invented and patented and largely advertised, but they all possess certain serious disadvantages. They are expensive, liable to go wrong, often clumsy and inconvenient, and while they do very well for ordinary 


\section{INTERNAL DERANGEMENTS OF}

walking and gentle exertion, they prevent the wearer from joining in the usual athletic exercises. To young patients they are particularly irksome, and even older ones complain of them, especially when they have to be worn continuously for some considerable period of time. As by a radical operation the surgeon at the present day is able to dispense with the use of trusses in cases of hernia, so in all

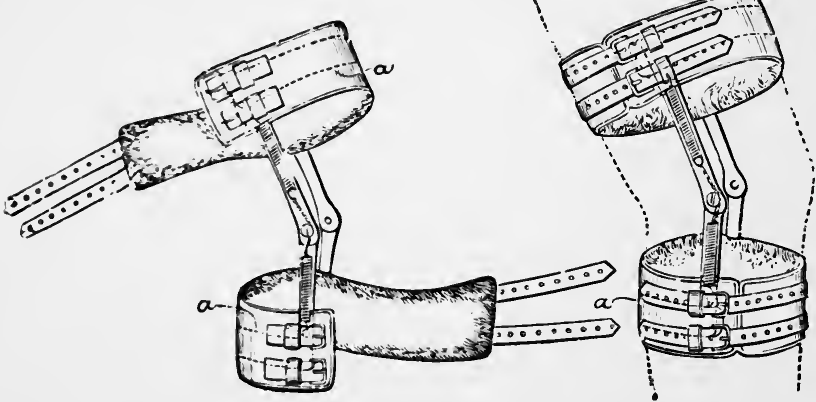

FIG. 59. The block represents a very useful apparatus to be worn by those cases unsuited for operation. It allows of flexion and extension of the joint, but no rotation, so that displacements of the semilunar cartilages are prevented. The block was kindly lent by Messrs. Lindsey \& Sons, instrument makers at Oxford and London.

probability will he be able, and that before long, to give up recommending trusses and other paraphernalia for the knee-joint in cases of displaced cartilage.

2. The operative treatment is, all circumstances considered, more satisfactory in this class of relapsing cases. A few years ago surgical opinion was about equally divided as to whether or no an operation was advisable; to-day most surgeons are in favour of it. 
Many circumstances have been instrumental in bringing about this change of opinion, and amongst these may be mentioned the improved technique in all surgical procedures, whereby the opening of a knee-joint has been robbed of that unspeakable dread which our forbears seem to have had for it. To-day the modern surgeon, although fully recognizing the risks of such a proceeding, is able to meet the difficulty with as much equanimity and confidence as he would any other major operation. And not only have the risks of disaster been lessened and almost abolished, but the better understanding of the precise pathology and anatomy of a displaced and torn cartilage has led surgeons to realize that without operation true union is in most cases not to be expected, and that at the best non-operative methods are but tentative. Furthermore, it is recognized that removal of the cartilage, if properly carried out, results in a permanent cure; so much is this the case that persons who formerly were dismissed the services as suffering from an incurable form of lameness are now, thanks to a skilful operation, enabled to remain. Many athletes have been enabled to resume their former games without any recurrence or any semblance of it. It may be stated generally that here, as in all other serious operations, the pros and cons should be carefully weighed so that none but suitable cases and those likely to be cured should be subjected to operation.

It may be premised that any operation other than removal of the displaced or free portion is unlikely to succeed. The method of suturing the cartilage to the tibia, as advocated by some writers, invariably fails, and it is difficult to explain why such an opera- 
tion is still recommended except on theoretical grounds. Every case of this plan of treatment that has been brought under the writer's notice has proved a failure. It is only those parts that are loose and

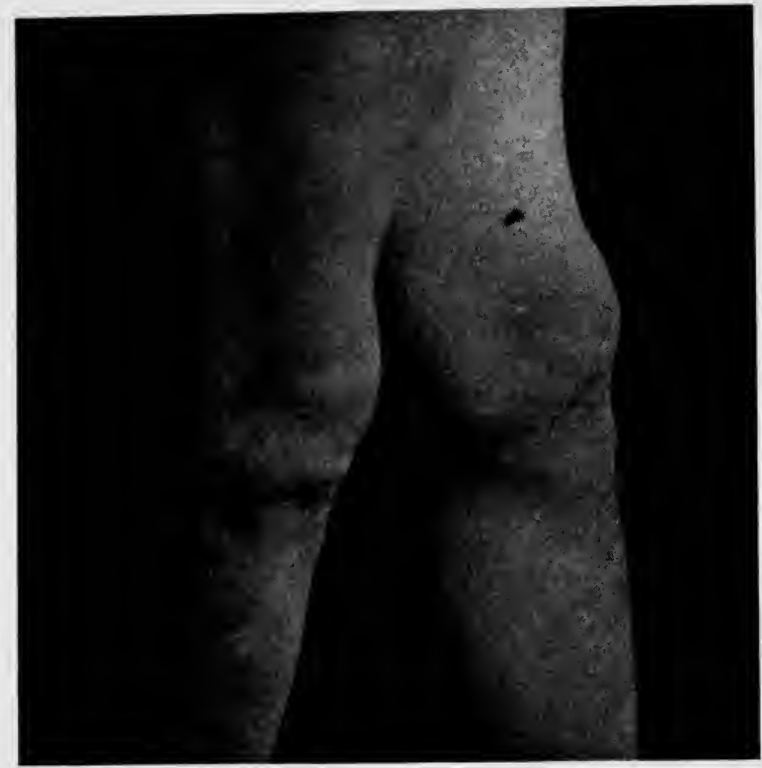

Fig. 60. A recent photograph to illustrate the scars of the transverse incisions formerly used by the author. The case is mentioned in the text. At a single sitting portions of both internal and one external semilunar cartilages were removed for displacement more than eleven years ago.

movable that should be removed, and it may be stated with a degree of confidence that the removal of a portion or the greater part of either cartilage will in no way diminish the usefulness of the joint. Twelve years ago the writer himself removed (at one sitting) portions of one external cartilage and both the internal cartilages from a patient, who is now 
able to ride perfectly and to play polo with great skill. Previous to operation he was unable to walk or take any exercise, as the least movement produced a displacement of one or other cartilage.

Preliminaries to operation. Success will depend upon certain details being carefully and sedulously carried out. First and foremost it is advisable that no operation should be undertaken while signs of serous effusion exist; it should be postponed, if possible, until all has disappeared. The patient should not be permitted to walk for three or four days immediately preceding the operation, and during this time should be kept resting in bed or on a sofa. Every attention must be paid to cleansing and disinfecting the skin of the knee and adjacent parts, which should also be clean-shaved. The skin of the thigh, knee, and leg should be daily washed with a solution of ether soap, followed by antiseptic compresses, and every means should be taken to secure absolute cleanliness. It is advisable that the surgeon and his assistant should wear sterilized rubber or other gloves, and every means be taken to ensure complete asepsis before, during, and after the operation.

Operation. The limb having been prepared, the surgeon stands on the outer side of the patient with his assistant facing him. The limb is slightly flexed and placed on its outer side, resting on a sandbag or other support. A slightly curved incision is made through the skin and fascia, beginning over the most prominent part of the internal condyle of the femur, and brought downwards and forwards to about threequarters of an inch from the inner margin of the 


\section{INTERNAL DERANGEMENTS OF}

patella, and then downwards and slightly backwards to the tuberosity and inner surface of the tibia, the lower limb of the incision being made parallel with the anterior portion of the internal lateral ligament (vide photograph). The middle point of the incision

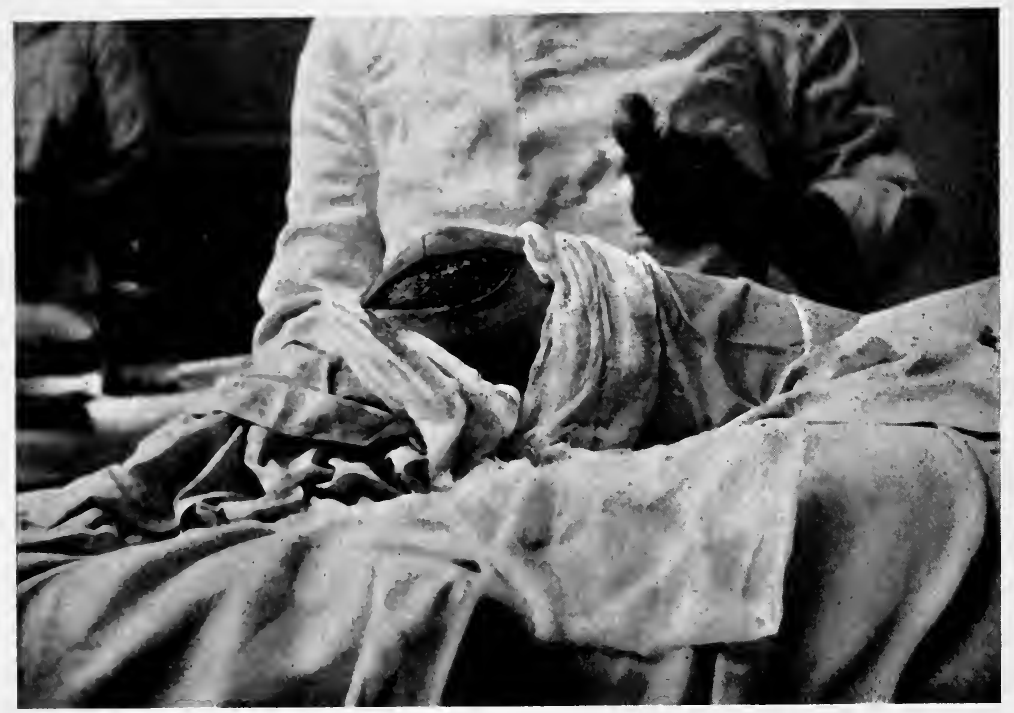

FIG. 61. Incision for exploratory arthrotomy of the knee-joint or for removal of a detached internal semilunar cartilage. First stage, through the skin and fascia. exposing the internal lateral ligament. In this position (slight flexion of the joint) of the limb the edges of the wound readily fall apart and do not require retraction.

should correspond with the line of the joint. The sides of the incision are then retracted, while all bleeding points are carefully secured and ligatured.

The internal lateral ligament should then be defined and opened somewhat obliquely so as to be exactly parallel with the direction of its fibres. It is customary at this stage to meet with the branches of the upper and lower internal articular arteries. 
Branches may be seen coursing under the periosteum over the head of the tibia. If not by this time cut: across they may be picked up and divided between two pairs of forceps and ligatured. The opening through the internal lateral ligament is best made

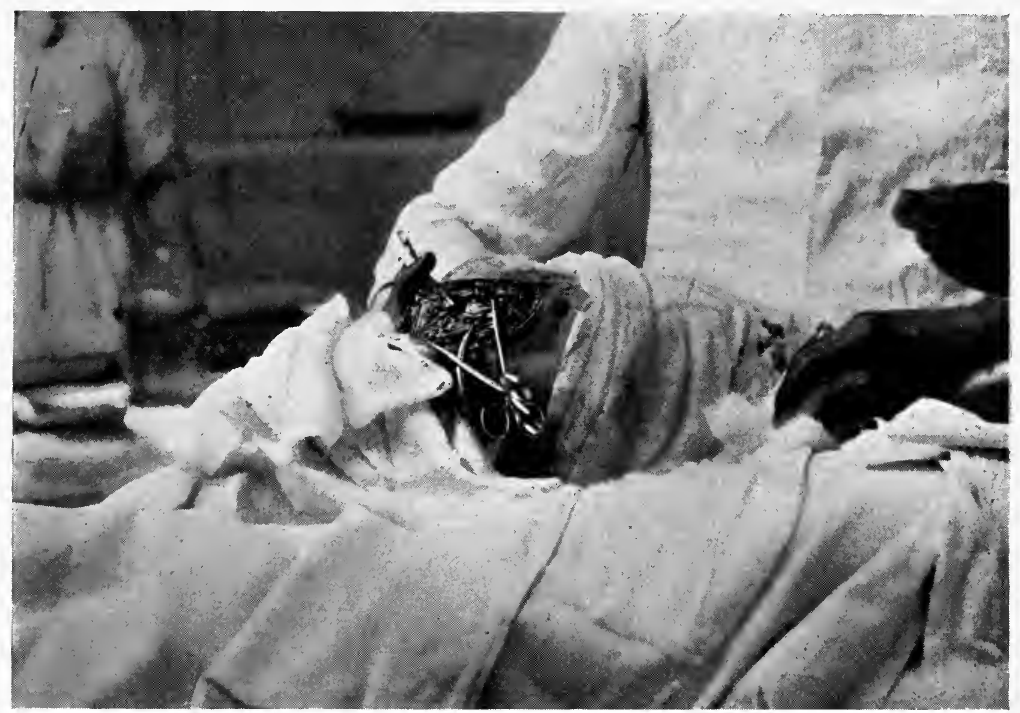

Fig. 62. Second stage in the operation for removal of a detached internal semilunar cartilage from the knee. The internal lateral ligament has been opened parallel to its fibres and its edges retracted. The synovial membrane has been opened parallel with the incision in the ligament, thus exposing the edge of the internal condyle of the femur. The thumb in the figure is represented as grasping a pair of forceps which is holding up the edge of a displaced cartilage. The other forceps are attached to the edges of the internal ligament and are being used as retractors.

three-quarters of an inch from the border of the patella. The cut edges of the internal ligament are now retracted so as to expose clearly the synovial membrane. All bleeding points should be ligatured before the joint is opened. A tourniquet is never required. The membrane may be divided trans- 


\section{0 \\ INTERNAL DERANGEMENTS OF}

versely or vertically, though the vertical incision is perhaps preferable, and the articular surfaces of the joint then come into view.

By drawing the patella outwards the internal cartilage and the infra-patellar pad (ligamenta

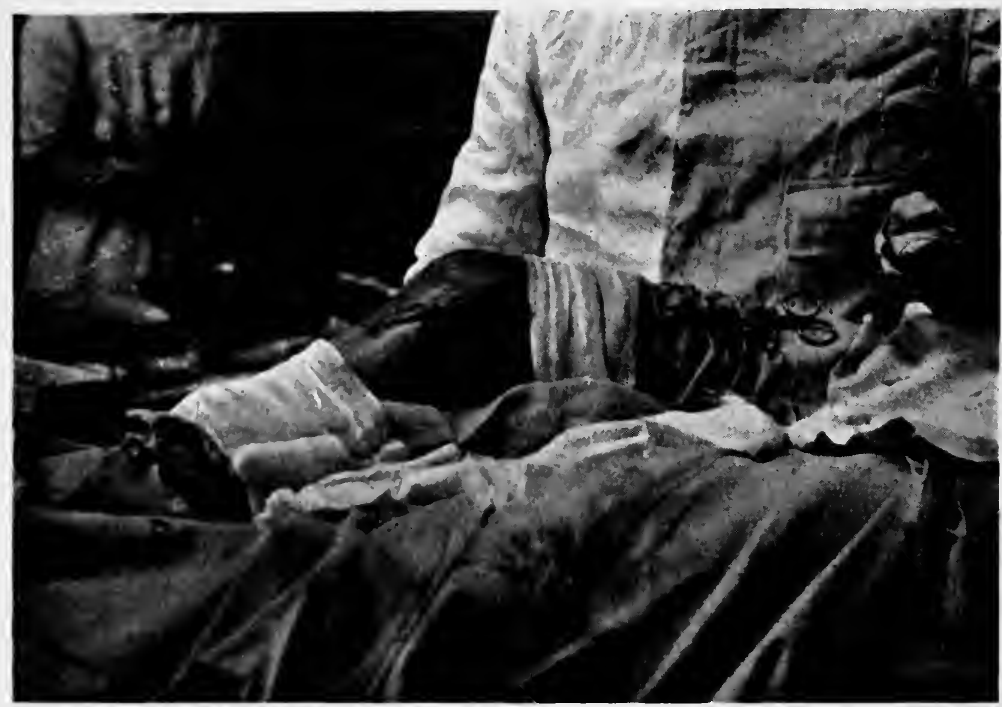

FIG. 63. The third stage of the author's operation for removal of a detached semilunar cartilage. It shows the wound elosed with a continuous silkworm gut suture and a wick of gauze inserted at the level of the eartilage for drainage. The drain represented in the figure is too long, and should be removed twenty-four hours after operation. The extent and direetion of the incision are well shown.

mucosa et alaria) are brought into view and may be thoroughly examined. The inter-condyloid notch should in all cases be carefully explored, for into this particular situation the cartilages are most frequently displaced, and here also other loose bodies, whether free or pedunculated, find a resting-place, The displaced cartilage, whether torn or merely 
loosened, is then seized with catch forceps, brought into the space bounded by the edge of the patella, tibia, and femoral condyle, and snipped off with scissors.

As much of the cartilage as is loose should be removed and no more.

When more than the anterior portion of the cartilage is loose and has to be removed, it may be necessary to enlarge the incision backwards by dividing the posterior fibres of the internal lateral ligament transversely. This should be avoided whenever possible, as the joint is liable to be weakened afterwards by the giving of the scar tissue even when the union has been satisfactory at first. There are surgeons who advocate a transverse division of the ligament, but it is obvious that however good the union may be it will be liable to yield when strain is put upon it later.

The offending cartilage having been removed and all bleeding stanched and blood removed from the joint, a digital exploration of the other cartilage and synovial folds should be made, on the same principle as abdominal explorations are recommended as a routine after laparotomy.

Thickened synovial fringes and folds, and even pedunculated bodies, are not uncommonly met with in long-standing cases, and, unless they are removed at the time, will require subsequent treatment. It is a good plan always to drain the joint for twentyfour hours. ${ }^{1}$ Most surgeons do not drain; formerly

1 The advisability of the practice of digital exploration of the knee-joint, as well as that of employing drainage after arthrotomy, has been questioned. As regards the former practice, the writer 
the writer did not, now he invariably does. He has found from experience that unless the capsule is drained, in nine cases out of ten it becomes rapidly distended, usually with thin slightly viscous bloodstained synovial secretion, sometimes with a thick tenacious viscid material not unlike boiled sago. Again, should any bleeding or oozing of blood take place into the joint after the operation it will be readily got rid of. Every surgeon must be aware that the presence of blood in a synovial cavity such as a knee is a serious complication when considered in the light of after-treatment.

Furthermore, pain is much less when drainage is employed, and if we compare the temperature charts taken of cases treated in all other respects in exactly the same way with this single difference, we may note that in those that are drained there is about one degree less, showing that the absorption from the joint and the tension are less. A thin gauze drain is inserted and the membrane and internal lateral ligament closed around it with catgut sutures. The skin is sutured with silkworm gut. An absorbent dressing is applied, and over this a tight bandage, and the limb retained in the slightly flexed position between sandbags, but without a splint.

If the pain is severe during the first night, aspirin considers that no satisfactory exploration or examination is possible without it. As to the latter, he sees no reason for making any alteration. In his hands, after a considerable operative experience, there has been no contretemps, no cause for regret; and he regards the objections as ex cathedra and theoretical. The reasons for his departure from the views generally in vogue at the present time as regards these details are pointed, and are given fully in the text. 
(grs. 10) may be given, or an injection of morphia (gr. $\frac{1}{4}$ ). Twenty-four hours later the drain should be removed and an absorbent dressing re-applied. On the eighth or ninth day the stitches should be taken out and a collodion or celloidin-gauze dressing substituted for the absorbent dressing.

The patella should now be moved from side to side as it lies in the trochlea, but it is advisable to defer other formal passive movements and massage of the knee until a few days later.

In about fourteen days, if the wound is firmly healed, the bandage may be discontinued at night, re-applied in the morning, and the patient allowed to get out of bed and walk on crutches. All movements may now be permitted, but the patient should not bear the weight of the body on the affected leg for at least two weeks. In six weeks the patient should be quite well and able for most things. The results are usually excellent, and it is quite exceptional to meet with a patient who is not completely satisfied. A recent case was able to play cricket in the University freshmen's match six weeks after the above operation had been performed. ${ }^{1}$

To ensure lasting success the patient must be encouraged to persevere in carrying out all his movements and not to rest satisfied until he is capable of doing everything he was able to do before the accident occurred. When walking is first tried the knee should be held stiff while the soles of the feet are placed flat on the ground.

1 The last four cases done by the author left hospital in fourteen days, walking well. 
Figs. $64,65,66,67$ are charts taken from recent cases in which one or other semilunar cartilage had been removed from the knee by arthrotomy, and after a complete digital exploration had been made. In Figs. 65 and 67 some fringes were clipped away in addition. Note the difference in the temperatures of 64 and 65 ,

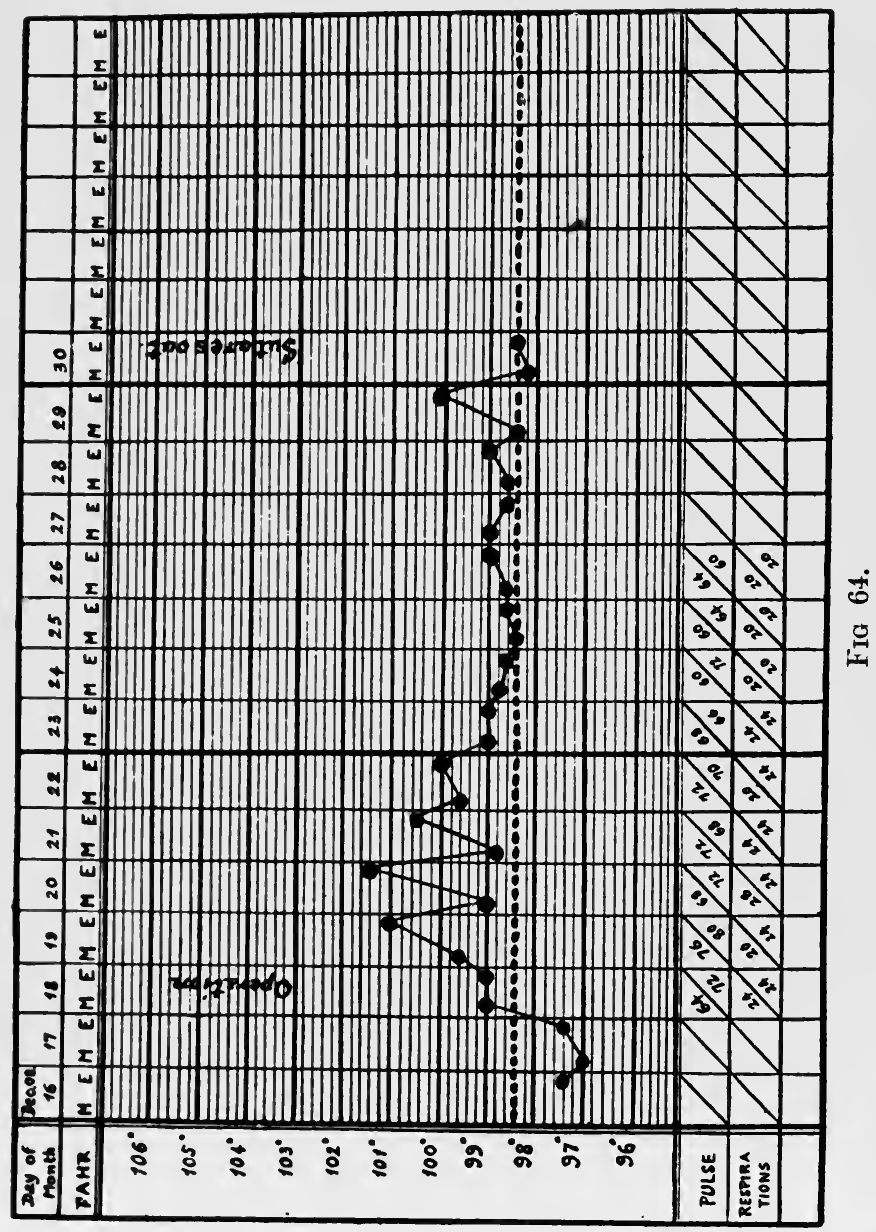


as compared with those of 66 and 67 . In the former no drainage was used, in the latter a gauze wick was inserted and removed twenty-four hours later. In every other respect the cases were treated alike. These charts are typical of what the surgeon should expect when operating with a strictly aseptic régime.

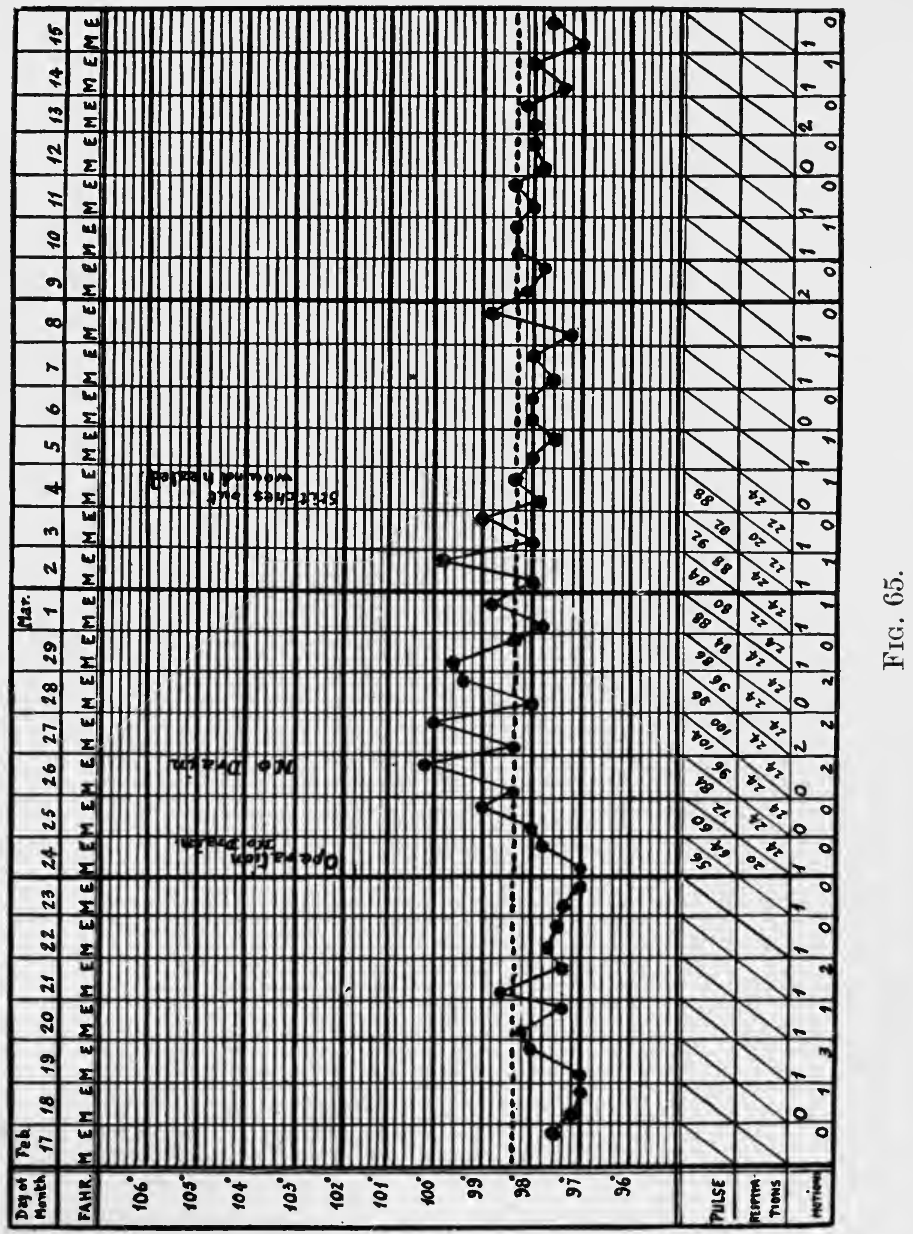


Figs. 64, 65, 66, 67 are eharts taken from recent eases in which one or other semilunar eartilage had been removed from the knee by arthrotomy, and after a eomplete digital exploration had been made. In Figs. 65 and 67 some fringes were clipped away in addition. Note the difference in the temperatures of 64 and 65,

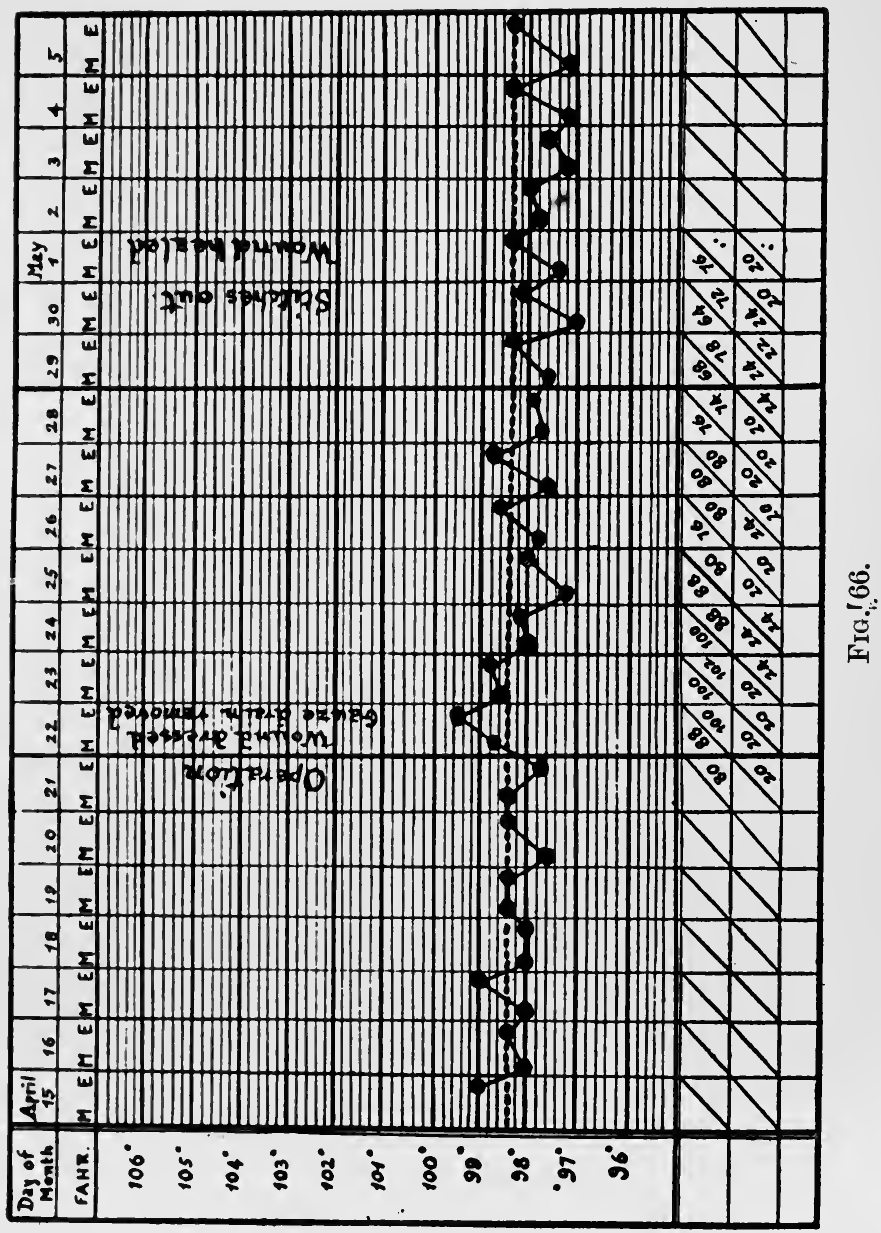


as compared with those of 66 and 67 . In the former nodrainage was used, in the latter a gauze wick was inserted and removed twenty-four hours later. In every other respect the cases were treated alike. These charts are typical of what the surgeon should expect when operating with a strictly aseptic régime.

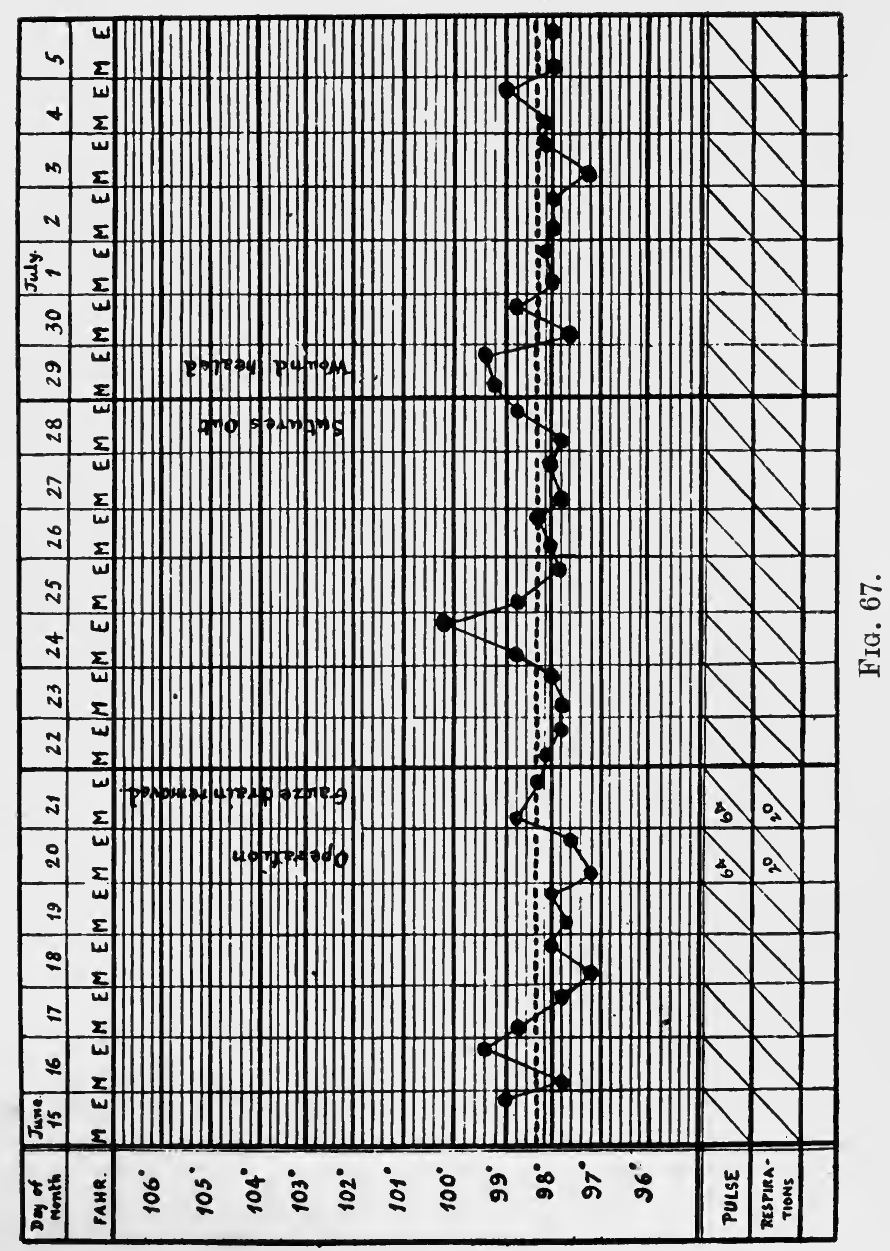


Displaced external semilunar cartilage. There are several cases of displaced external semilunar cartilage on record, in most of which the detachments were situated anteriorly ; in one or two exceptional cases the posterior horn was ruptured. The tendency in most of these was for the displacement to be inwards towards or into the inter-condyloid space. Mr. Annandale ${ }^{1}$ relates a case in which the external cartilage could be seen and felt displaced. Mr. Godlee $^{2}$ showed a specimen taken from the dissecting-room, in which the external cartilage had been torn from its circumference, and lay in the intercondyloid notch. Mr. Langton ${ }^{3}$ mentions a case in St. Bartholomew's Hospital of a loose body in the left knee. The external semilunar cartilage had been torn from its posterior attachment and was further detached laterally for half or more of its length. Such cases, for anatomical reasons, are extremely rare. The writer has only met with three ${ }^{4}$ authenticated

1 British Medical Journal, February, 1887, p. 319.

2 Transactions of the Pathological Society, vol. xxxi, p. 240.

3 Transactions of the Pathological Society, 1888, p. 282.

4 Since the last edition was published, still another case has come to hand. A gentleman while playing football stepped upon the heel of another and was violently thrown. 'He felt something give, suffered much pain, and was quite unable to straighten his right knee.' That evening the joint swelled up. He was treated by rest and bandaging, and recovered satisfactorily up to a certain point. As soon as he began to walk, however, the displacement recurred, accompanied by all the former symptoms. He was sent to the writer, who opened the joint and found a detachment of the anterior third of the external semilunar cartilage from the margin of the tibia. The anterior horn was intact, but the loosened part of the cartilage was displaced inwards and lay between the bones. The detached portion was excised, and the patient made a good recovery. 
cases in which the external cartilage had been detached; one occurred in the practice of a friend, another in a patient from whom he had also removed both internal cartilages. In these two cases the displacement was into the inter-condyloid space and resulted from a laceration near the junction of the anterior and middle thirds which allowed the posterior segment to alter its position. The third case occurred in a youth who twisted his knee as the result of a fall from a ladder. The accompanying photograph shows the condition of his limb before operation. He stated that he did not know what happened to his knee, except that he 'felt something snap', and 'suffered agonies of pain'. His knee immediately became swollen, and he was unable to put the foot to the ground, nor was he able to keep his knee fully extended. He was kept by his medical attendant in bed for three weeks; at the end of this time, although most of the swelling had disappeared, he was unable to put his foot to the ground without pain, and he could not stretch the limb to its fullest extent. For over seven months he was unable to walk any distance without considerable pain. His occupation was that of a milkman, and finding an increasing difficulty in doing his work, he applied to the Radcliffe Infirmary for treatment. On examination, the knee was found slightly bent and could not by any means be fully extended. There was marked atrophy of the muscles on the proximal side of the joint, and though a thickening of the synovial membrane was evident, there was no distinct synovitis. He complained that, besides the joint being painful, it was apt to swell if he 
walked more than a few hundred yards. The photograph shows plainly the posture of the limb and
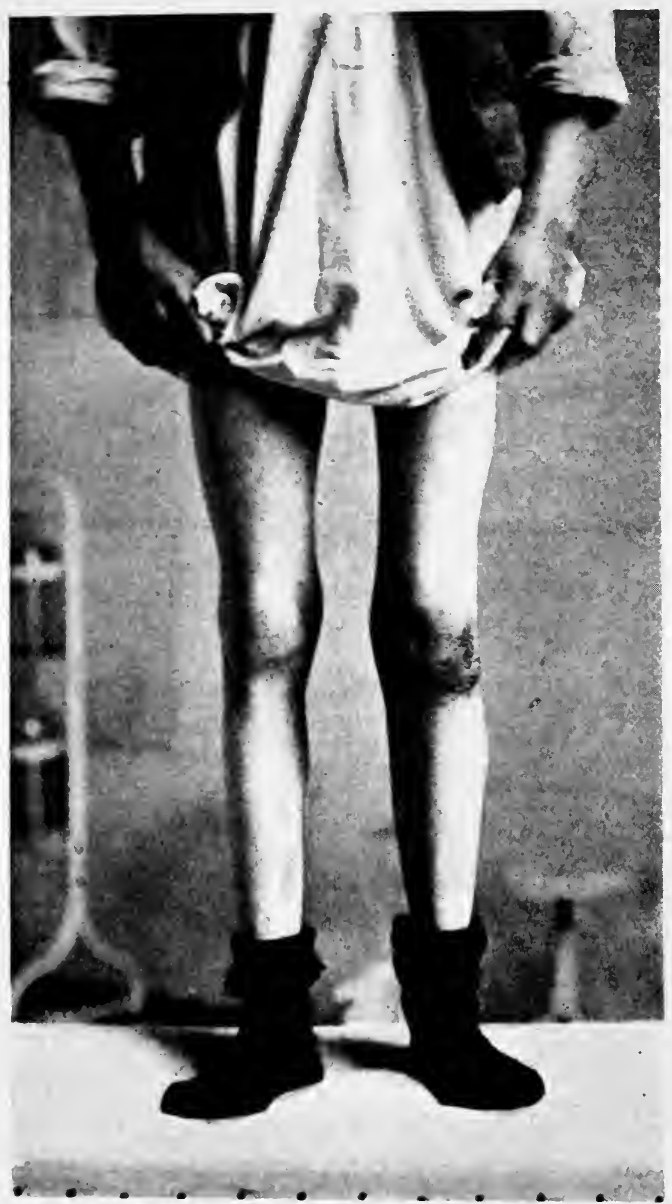

Fig. 68. Case of long-standing displacement of left external semilunar cartilage described in text. Note atrophy of muscles of thigh with commencing hypertrophy of those of the calf ; also flexion of knee and foreshortening of limb. The discoloration shown was due to former blistering.

atrophy of the muscles. After due preparation, the knee was opened by a vertical incision corresponding 
with a line over the centre of the base of the cartilage. The skin, the superficial fascia, and the capsule were divided in turn, the joint opened and the cartilage exposed. It was found to have been torn across at its posterior extremity, and as some of the coronary ligaments had been detached, the cartilage thus loosened could be caught between the bones in certain movements of the limb. The movable part of the cartilage was excised and the capsule sewn up with catgut, a gauze drain inserted, and removed twenty-four hours later; the skin sutures, of silkworm-gut, were removed on the ninth day. The patient left the hospital in three weeks. At the end of five weeks he was completely cured as far as movements were concerned, and able to do his work as if nothing had happened. Regeneration was taking place quite satisfactorily in the wasted muscles of the thigh when he was last seen a month ago (four months after operation).

From his experience of these cases the writer is of opinion that when the diagnosis can be made, especially if recurrences are frequent, the above operation is the best method of treatment.

Since the above account was written the author has met with two more cases, making five in all. The last one, a photograph of which is appended, occurred in a schoolboy aged sixteen who wrenched his knee awkwardly at football. The diagnosis was made from the history of the accident, the excessive tenderness over the line of the cartilage, together with the characteristic posture of the limb; the joint could not be extended fully. Reduction was performed by manipulation under complete anæsthesia, and 
the joint was subsequently treated as an ordinary sprain.

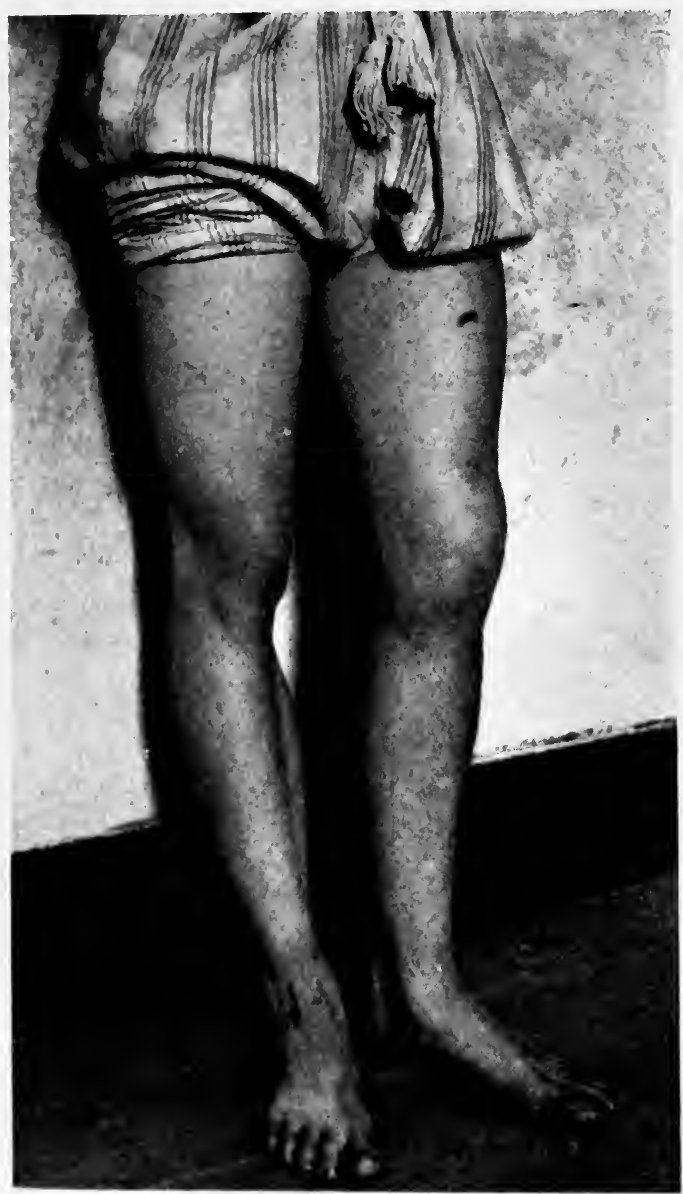

Fig. 63. Photograph showing characteristic flexion and swelling in left knee-joint, from a recent case of displaced external semilunar cartilage. Note posture of limb as identical with that found in displacement of internal cartilage. Diagnosis was subsequently verified by operation after he had suffered a relapse.

Hypertrophied synovial fringes. In very chronic long-standing cases of recurrent synovitis, it occa- 
sionally happens that the synovial pad (the ligamentum mucosum) behind the patellar ligament becomes thickened and hypertrophied, the ligamenta alaria on each of its borders become indurated, thickened, and may even present papillated fringes. As these structures increase in size, they are liable to be caught and pinched or bruised between the femoral condyles and the tibia. The bruising further stimulates their increased growth so that in time they form definite structures constantly liable to injury. When nipped, they give rise to a sudden pain and checking, rarely to locking, of the movements of the knee. As a rule when the condition exists by itself, it is suspected rather than diagnosed; an exact differential diagnosis being impossible. When once begun, the tendency is for the symptoms to increase rather than to diminish ; the disability, therefore, is a progressive one. The condition is best treated by opening the joint as for an exploratory arthrotomy and completely clipping away the whole of the infra-patellar swelling, being careful to see that no thickened folds of synovial membrane are left in the inter-condyloid space of the femur. The fringes on rare occasions develop pedunculated bodies which, although fixed or moored in the first instance, become detached later and form one variety of movable bodies in the joint. 


\section{CHAPTER VII}

\section{GENERAL INJURIES TO MUSCLES AND TENDONS}

Muscles and tendons are liable to injury in many ways, as the result of different forms of violence; thus they may be strained or sprained from overstretching, may become herniated or protrude through openings in the fascia enveloping them, displaced or dislocated from the grooves in which they normally course, or even partially ruptured or torn across. The tendons which surround a joint that is sprained or dislocated almost invariably suffer, though to a less extent than the ligaments. In fractures they are often seriously damaged.

Muscle sprain. When the fibres of a muscle or its aponeurosis or tendon are subjected to violent overstretching or partial tearing, it is said to be strained or sprained. This occurs very commonly in persons actively engaged in athletic performances, less frequently in those who follow laborious occupations. It is usually the result of sudden violence where a muscle or group of muscles is taken unawares, though in exceptional cases repeated muscular efforts may be accountable. Many examples may be given, such as 'rider's sprain' affecting the adductors of the thigh, 'jumper's sprain' the hamstrings or muscles of the calf, 'tennis elbow' the pronator radii teres, 'driver's or angler's elbow' the common 
origin of the supinator extensors of the fore-arm, ' labourer's back' with which the sacro-lumbalis is concerned, and 'bowler's side' affecting the oblique muscles of the abdomen. Each of these injuries, to which the popular name has been given, is an example of muscle injury. The symptoms are pain, tenderness on pressure, with inability to carry out the particular movement or movements concerned in the injury. As a rule, recovery is rapid, though in some cases the individual may not be able to resume his duties or to take part in athletics for weeks or even months.

The treatment consists in discontinuing movements which give rise to spasm or pain when a muscle or group of muscles is exercised. If the pain continues and the recovery is delayed, a good plan is to support the painful part with firm strapping, and to encourage the patient to use the muscles freely. Massage and electric vibration are helpful, and may be used when the strapping is removed for readjustment; or it may even be applied over the strapping.

The term 'strain', as in the case of articular injuries, is generally meant to indicate a disability of less severity than sprain.

Herniæ of muscles occur not infrequently among young and powerfully built athletes, and are the result of a sudden violent contraction of the muscle when carrying out some supreme athletic effort. The belly of the muscle, in shortening suddenly and expanding proportionately in width, bursts its way out and protrudes through its ensheathing fascia. As a rule, the cleavage in the fascia is in the longi02 
tudinal direction, but it may be transverse. In young cavalry soldiers, hernia of the adductor longus muscle is not infrequently met with. The recruits, often powerfully built men, trained to gymnastics or marching, are suddenly ordered to mount and to

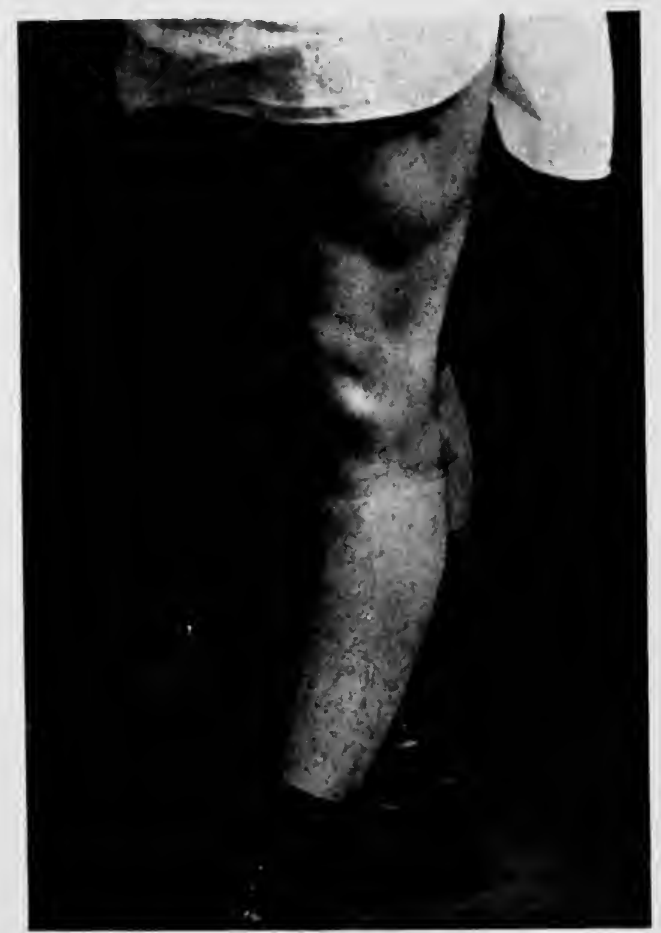

FiG. 70. Hernia of muscle in thigh, result of muscle sprain.

ride on barebacked horses, without stirrups. Unaccustomed as many are to riding, it is not surprising that, in their frantic efforts to escape falling off, they rupture the sheaths of the adductor muscles in gripping the horse. The tensor vaginæ femoris, the rectus femoris, the tibialis anticus, the rectus abdo- 
minis, have all afforded examples. Herniæ of muscles are comparatively rare, and may be recognized by the existence of a soft swelling in the course of the muscle, which is soft and prominent when it is relaxed, but becomes smaller and harder when it is made to contract. Often there is no pain and surprisingly little inconvenience; the patient presents himself mainly to ascertain the cause of the swelling, or for an explanation of a sensation which has been met with in a certain number of cases, and has been described 'as if water was trickling over the part'. This latter symptom is probably due to some interference in the course of a superficial nerve. A feeling of insecurity is occasionally complained of.

The diagnosis is easy, and in some cases where the hernia is small, little or no treatment is required. When the inconvenience is considerable, it may be necessary to operate. If the case is recent, and the split a longitudinal one, a curved incision through the skin and fascia is made so that its convexity is directed upwards over the course of the muscle, the flap so marked out is turned downwards so as to expose fully the rent in the fascia; the muscle is then put on the stretch to retract it. Any adhesions that are present must be freed, and the edges of the fascia brought together and sewn as accurately as possible. There may be considerable ecchymosis superficial to the fascia in the more recent cases. When the rent is transverse, as occurs in the abdominal rectus or in the long adductor of the thigh, it may not be possible to bring the edges completely together; in such a state of affairs it is well to draw 
the edges together as near as possible and to fix them as best we can with a stout catgut suture.

The after-treatment consists of resting the limb until the external wound is healed, and then in using gentle massage and encouraging slight active movements. The results are generally satisfactory, even where the suturing of the fascia has been incomplete. It has been suggested that the hernia should be excised; this is quite an unnecessary mutilation, and of very doubtful utility.

To prevent adhesion between the muscle fibres and the line of suture, the Faradic current may be employed with advantage, the movement occasioned by the contraction of the muscle tending to stretch or break down any adhesions that form.

Displacement or dislocation of tendons. Although many cases are recorded, it may be said that, speaking generally, displacements of tendons are rare. The most familiar examples are the dislodgement of the peroneus longus tendon from the groove behind the external malleolus on to its external or anterior surface, the tibialis posticus to the anterior surface of the internal malleolus from its place behind it, and the long head of the biceps flexor cubiti inwards or outwards from the bicipital groove of the humerus. The small tendons at the back of the neck are occasionally displaced over the posterior tubercles of the transverse processes of the vertebræ. In the majority of cases, notably in those of the peronei (longus et brevis) and the tibialis posticus, there is in the course of the tendon a sudden and abrupt deflexion around a bony prominence which takes the place of a pulley. The bony prominence in each 
instance is deeply grooved, so that the tendon lies securely and is roofed in, so to speak, by a ligamentous expansion or confining sheath. The biceps cubiti is displaced by the movements of the humerus

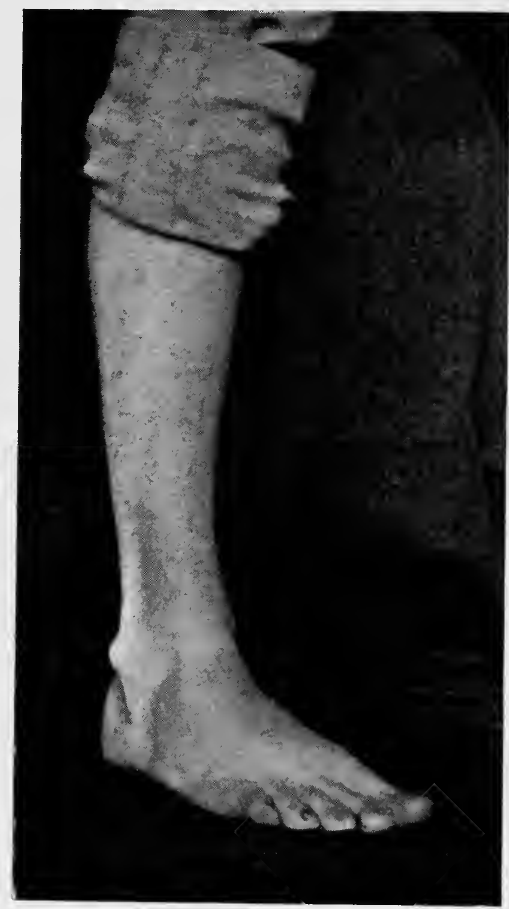

Fra. 71. Dislocation of the peroneus longus tendon. Note formation of false bursa between it and external malleolus. Taken from a schoolboy, aged 15. Was restored subsequently by operation by author's method. Vide text.

around it as it lies in a straight course in the bicipital groove. The peronei may be found dislocated in a patient who has sustained a violent twist or wrench of the foot.

'If, while the foot is either inverted or pointing 
straight forwards, the peroneus longus is made to contract, it cannot slip, for its tendon is tightly drawn into the bottom of the groove at the back of the external malleolus; but when the foot is everted,

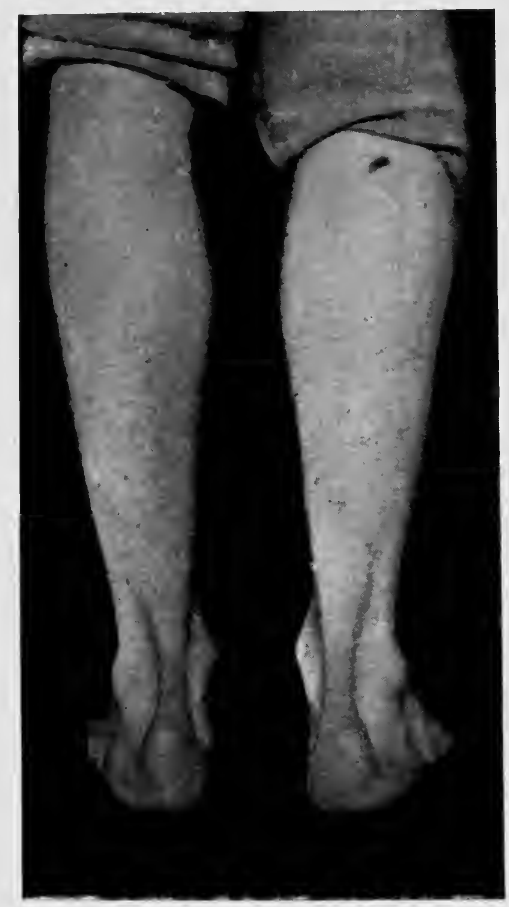

Fig. 72. Back view of displacement of both peroneal tendons (longus et brevis) on right side. Note the bursal enlargement over the external malleolus. The photograph is unfortunately not a good one.

the tendon has a tendency to leave its groove, so that its sheath becomes its pulley, and this, if the strain is severe, may give way.' (Marsh, ${ }^{1}$ Professor Howard.)

1 Clinical Essays and Lectures. J. \& A. Churchill, pp. 1-8. 
A schoolboy, aged sixteen, had been running in his stocking soles after a football ; he accidentally stepped on the ball, twisted his ankle and fell. When examined a few minutes later, there was a large extravasation of blood over the malleolus and on the outer side of the ankle; he complained of a sickening pain and inability to move his foot, and vomited. The tendon could be distinctly felt lying in front of the malleolus, even through the effusion, and was easily replaced by manipulation. The foot was then placed at a right angle to the leg, inverted, and fixed in this position by means of a moulded felt splint. After forty-eight hours, massage and gentle passive movements were begun (the tendon being retained in position during the movements by the fingers of the manipulator's other hand, while the foot was kept inverted), and continued daily. At the end of three weeks the splint was discarded, and as all swelling had disappeared, the ankle was strapped and the patient allowed to voluntarily exercise the muscles of his foot with the movements of flexion and extension. In four weeks he was able to walk, though he complained of a feeling of weakness in the ankle; in six weeks he was quite well and able to run. It is now over four years since the accident, and there has been no recurrence.

If the foot, instead of having been everted, had been inverted, the possibility is that he might have displaced the tibialis posticus, for in this position this muscle tends to leave the groove behind the inner malleolus where it is merely retained in position by the fibrous sheath upon which it pulls directly.

Fig. 73 represents such a case. The photograph was taken from the leg of a youth aged seventeen, who violently twisted his foot in stepping out of a window on to a croquet ball. The foot, on which there was only a thin shoe at the time, became suddenly inverted, "he felt something give at the inner side 
of his ankle, and suffered considerable pain.' After this the ankle became much swollen and he was unable to walk for some days. The true nature of the injury was not recognized at the time, so that when he presented himself for examination some

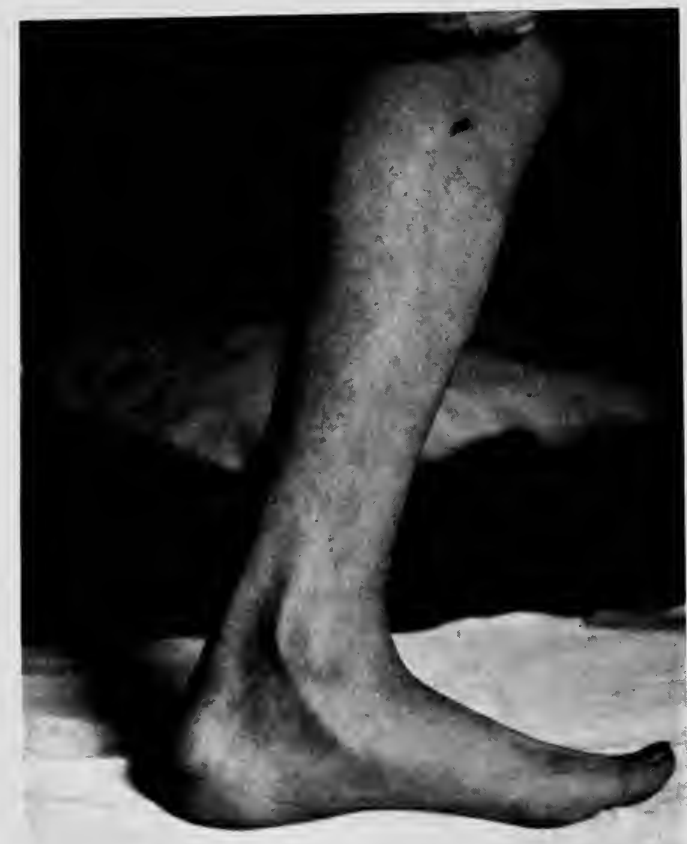

FIG. 73. Displacement of the tibialis posticus tendon on to the inner malleolus; the result of a twist of the foot while in the position of inversion.

months afterwards, he was able to produce the displacement of the tendon at will, and still suffered from a weak ankle.

The author has seen a case in which both the peronei tendons (longus et brevis) could be dislodged at will by the patient, 
a young lady, by simply everting the foot in a sudden and jerky way. The tendons jumped out of their grooves with a snap, occasioning very little pain but producing complete disablement. All discomfort and crippling disappeared as soon as the tendons were replaced, and this could be readily and easily performed by gentle manipulation. For years the patient treated the matter with indifference, until finally the recurrences became so frequent as to be annoying. The ordinary means of retaining the tendons by strapping and wearing a special appliance succeeded so long as they were persevered with, but as soon as they were left off recurrence took place. Accordingly, it was deemed advisable to do away with the difficulty, and an operation was performed. A curved incision was made over the outer malleolus and side of ankle, the flap turned upwards and the groove in the fibula exposed. This groove was found to be shallow and almost completely filled up by portions of the sheath ; all this tissue was cleared out, the groove deepened, the tendons replaced, and the remains of the sheath stitched firmly and accurately over them with catgut. The ankle was put into a moulded splint and, as soon as the skin stitches had been removed, at the end of eight days, passive movements and massage were begun and practised two or three times a day. The result was in every way satisfactory.

As recurrence seems, for anatomical reasons, so liable, immediate operation appears to offer the best prospect for a permanent cure, contra-indications such as enfeebled health and old age being alone considered. Given a young, athletic, and energetic person, an open operation gives far the best prospect of satisfactory treatment; the same means may be employed in the case of displacement of the tibialis posticus.

The long tendon of the biceps has on many occasions been displaced from its groove, either out- 
wards or inwards, generally inwards, as a result of violent or repeated movements of the upper arm, such as are made use of in wringing clothes; the sheath which converts the groove into a tube is the tendinous expansion of the pectoralis major, and when this is torn across the tendon slips out. The patient suffers pain, is unable to extend the fore-arm until the tendon has been replaced by abducting the arm and manipulating it. - The displacement may often be produced voluntarily, and in such cases needs no special treatment. Reduction may be brought about by manipulation; a good plan is to direct the patient to place his hand on the shoulder of another person and, while it is thus abducted, to advise him to carry out the movements of rotation first in one direction and then the other.

The small tendons at the back of the neck are not infrequently displaced, resulting in temporary locking with pain, which is sometimes very acute, and centred at the exact spot. Such accidents occur from some sudden and awkward movement of the head, as in turning hurriedly to one side or the other.

An example would perhaps better explain matters.

A young man, leaning out of an upstairs window and talking to his companion in the garden below, was suddenly startled by the banging of a door behind him. Turning his face to the right sharply, he heard a ' click', felt an agonizing pain in the right side of his neck, found his chin approximated to his right shoulder and fixed in that position. Every attempt at straightening the neck or moving his head to its proper position was impossible, by reason of the pain, which was most intense. It was possible that a small tendon had been displaced, and presumably a portion of the splenius capitus or other muscle had become caught and hitched over 
the posterior tubercle of one of the transverse processes of an upper cervical vertebra. ${ }^{1}$ Holding his head between his hands, and with an expression of mingled fear and anxiety, he presented himself, saying: 'For God's sake, doctor, be quick, for I've either broken my neck or slipped it out of joint!' It was no easy matter at first to assure him that there was no real cause for his anxiety. Nitrous oxide gas was administered, and by means of gentle rotation, combined with to-and-fro movements of the neck, the trouble was after some difficulty overcome. As soon as the patient recovered from the anæsthetic he volunteered the statement that he was absolutely free from any pain.

A few days ago an undergraduate arrived with his head turned to one side so that the vertex was approximated to his left shoulder; he complained of acute pain whenever he attempted to straighten his neck, or indeed to carry out any movement other than that of bending his head towards the left shoulder. He stated that while swimming in a bath, engaged in a game of water-polo, he turned his head suddenly to the left in endeavouring to see the ball ; felt something snap, and believed that he had dislocated his neck. A careful examination elicited that he had pain on pressure over the fourth and fifth cervical vertebræ posteriorly, which was intensified with any attempt at movement. By gently massaging and stroking the muscles of the back of the neck on both sides of the spine, the local pain was in a short time considerably diminished; then standing behind the patient, who was seated on a low chair, and laying hold of the painful place with the left hand and approximating the left side of his face to his shoulder so as to relax the muscles on that side, reduction was brought about by a gentle rotation of the head from left to right. While the movements of rotation were being executed, the muscles at the back of the neck were manipulated with the fingers of the left hand. Pain

1 The view has recently been advanced that some at least of these cases are in reality subluxations at the cervical joints rather than displacements of tendons, and certainly with some reason. 
was immediately relieved and the patient walked out apparently well. On the day after, he was again seen, and complained of nothing more than a slight tenderness over the left side of the neck; no fixing apparatus was thought necessary, and the case did well. Other similar cases are on record : one in which a jockey, in riding home an easy winner, was incautious enough to turn suddenly his head over his shoulder that he might see the other riders. His head became fixed in this position and he rode home past the winning post still looking backwards, much to the astonishment of the onlookers! On dismounting, many attempts were made by friends and admirers to rectify matters, but their efforts were all to no purpose. A surgeon coming on the scene, with the aid of some chloroform, enabled him to fulfil his engagement to ride another race, which he did, history relates, again with success.

Some of these cases which were formerly thought to be mere displacements of tendons in the neck, back, and loins, are now believed to be cases of partial dislocation. A few that were at first regarded as sprains have been proved by radiography to be genuine cases of fracture (vide pp. 207, 208, report of cases of fracture of the transverse processes of the lumbar vertebræ).

On a few occasions, hospital patients of the labouring class have applied for assistance with the history of having ' ricked' the muscles of the back, either in lifting or in slipping awkwardly while carrying loads either up or down a ladder. These disabilities were until quite recently regarded as mere displacements of portions of the sacro-lumbalis muscle, since they were generally relieved by the administration of an anæsthetic aided by deep rotatory manipulation and kneading. Indeed, these simple expedients were 
usually so eminently successful that they recalled to one's mind in a forcible way the fabulous results achieved by the old time bone-setters who, by their various manipulations, wrought miracles, but with this difference, that in these particular cases there is genuinely a 'something out of place'.

The after-treatment is usually simple. There is seldom any sign of hæmorrhage or ecchymosis, the pain being immediately relieved by the manipulations; only a certain degree of tenderness remains which may last for three or four days. A bandage or some adhesive strapping may be applied over the seat of mischief, more as a placebo than a necessity.

Recently radiography has proved that some of these cases which were thought to be sprains are in reality cases of fracture of the transverse processes of the lumbar or dorsal vertebræ. S. Lange ${ }^{1}$ records the case of a commercial traveller, aged fifty, who in lifting a heavy tray out of a trunk felt something give way in the small of his back. Great pain followed, and compelled him to cease work. Next day, when bending over a washstand, he felt excruciating pain in the same place and fell to the floor. After this he could do no work by reason of the pain, especially in stooping, riding on cars, and walking fast. When in bed he suffered occasional twinges of pain. Examination showed an area of tenderness the size of a dollar on left of the last lumbar vertebra, in the angle between the crest of the ilium and spine. To this spot all pain was referred. It resisted all treatment, such as rest in bed, strapping, salicylates, potassium iodide. Skiagraphy showed a frac-

1 New York M $\epsilon$ d. Journal, Oct. 9, 1909, p. 691. 
ture of the left transverse process of the fifth lumbar vertebra. The process was detached and tilted up and showed an irregular outline. The bone was evidently broken by violent contractions of the patient's powerful erector spinæ muscle. Another case is reported by the same writer, in which the transverse process of the first lumbar vertebra was fractured by the direct blow of a crank. There was marked rigidity of the muscles over it, and tenderness on pressure. X-rayś displayed a fracture of the transverse process with upward displacement of the fragment. These cases reveal a new cause for chronic lumbar pain. Fracture of a transverse process of lumbar vertebra has been considered rare. In March 1908, Ehrlich, ${ }^{1}$ reported what he considered the first case of isolated fracture of the transverse process of a lumbar vertebra diagnosed during life. Three post-mortem cases had previously been recorded.

In December 1908, Haglund ${ }^{2}$ added some other cases in which the diagnoses had been made by X-rays. The causes were related as being-

1, Coal bucket fell on lumbar spine.

2 Cases occurred while riding a bicycle.

1, While a man lifted his son.

1, Patient fell downstairs.

1, An athlete who frequently fell on his back.

The frequency with which the symptoms of chronic sacro-lumbar pains occur and the difficulty of diagnosis, give much clinical importance to this

1 Deutsche Zeit. fïr Chirurgie, p. 413.

2 Ibid., cxvi, p. 321. 
fracture. If unrecognized, many are thought to be sprains, and non-union, or delayed union will follow, because the fragment will move with every movement of the body. The pain may persist and become chronic. In some cases the patient inclines his body towards the fracture. Diagnosis is made by the history of accident, tenderness on pressure, pain varying in intensity, increased in walking and stooping, relieved by lying down, and attendant muscular rigidity. X-rays alone can show the fracture. Prognosis is important from medico-legal standpoint and compensation. Treatment, by strapping and rest.

Various other tendons in the body have been at times displaced, but the occurrence is so rare that little notice need be taken regarding them here. The principles of treatment are the same for all.

Rupture of muscle or tendon. A muscle or tendon may be ruptured in almost any part of its course, either in its continuity or near to its points of attachment. In the greatest number of cases it occurs where the muscle fibres join with the tendinous. Each individual muscle would seem to have its weak spot, and rupture takes place with remarkable regularity at certain definite situations. Degenerated and enfeebled muscles are said by some authorities to be no more prone to rupture than are the muscles of healthy and vigorous persons.

Tearing of muscle may result from outside injuries, such as blows and crushes, but is more generally occasioned by sudden, indirect, and involuntary forms of violence. When rupture takes place in the belly of a muscle, the fibres contract and retract, 
the amount of separation depending upon the length of the muscle between its proximal and distal attachments, as well as its limitations by lateral or other attachments, to a bone or neighbouring aponeurosis.

The long muscles in the extremities furnish the best examples of separation after tearing. When a muscle is torn, the space between its retracted ends becomes rapidly filled with blood, which is usually venous though sometimes arterial. The hæmorrhage may be copious, and give rise to much swelling. In all cases, however slight, there is some bleeding; this is an important fact in the matter of treatment. The blood clots and forms a temporary bridge or platform between the divided ends; this is ultimately replaced by fibrous tissue which in the end forms a link of union between them. Not infrequently, when a tear takes place through the belly of a long muscle, the fibres of the proximal end are curled upon themselves within the sheath of the muscle, and form adhesions in faulty positions; or may even become tucked up behind and give rise to increased shortening and a proportionately longer interval. The distal end of a torn muscle or tendon invariably undergoes degeneration when its nerve of supply is cut off. More frequently, muscles are only partially torn across, and we meet with every degree, from laceration of a few peripheral bundles of fibres to complete severance of the whole muscle. Speaking generally, partial lacerations are found in the short and flat muscles of the body, while the long muscles of the extremities afford examples of the complete. Instances of complete rupture are found amongst 
those which have been brought about by sudden and unexpected contractions. Athletic exercises produce many, so much so that a considerab!e number of these injuries have derived popular names from the particular form of sport or exercise in which they occur; thus we have 'bowler's side', 'rider's sprain', 'lawn-tennis leg', 'cricketer's shoulder', and 'sprinter's thigh'. The clinical symptoms are characteristic and alike in both groups. There is usually a history of sudden pain localised in some definite spot, with the sensation as if struck by a whip or a stone, or as if something was torn. A distinct snap may be heard and the limb becomes powerless. At the seat of rupture there is tenderness and swelling. When the swelling is insignificant, and the separation great, a distinct gap may be felt in the muscle, which increases in width whenever it is made to contract. The treatment is therefore indicated according to the importance of the particular muscle involved, and to the degree to which it is incapacitated. An important muscle, such as the quadriceps extensor of the thigh, may require an immediate operation, whereas a small one like the plantaris may be treated equally well by posture and strapping. Approximation of the fibres is of the first importance, and whilst in the one case this may be best carried out by an open operation with careful suturing of the fibres and their sheaths with catgut, in the other, bandages, strappings, and special contrivances will be all that is required. Primary and immediate suture, if effected within a few days, gives far better results than secondary suture at a later period. Partial ruptures 
result from the same kind of injury as complete, and give rise to the same inconveniences, though to a less degree. There is pain and tenderness, and even a slight depression or gap may be left.

For treatment, while absolute rest is not recommended, the requirements will in most cases be readily met by strapping, so as to support the injured part, assisted at the time, or followed later, by rubbing and active movements.

Athletic exercises are responsible for lacerations in almost all the muscles and tendons of the body at some time or other, and more especially is this the case with those who attempt violent athletic exercises without first training these structures gradually. The system of training here referred to is that of gradually increasing exercise, and has no necessary regard to dietary or other considerations. At the public schools and universities, where athleticism is a recognized accomplishment, and where youth and early manhood, and to a limited extent early middle life, enter into real competition, there is no kind of injury so commonly met with as that of sprained, overstretched, dislocated or lacerated muscles or tendons. During an extensive experience of nearly twenty years at one of the old universities, the writer has come to the conclusion that, for some reason or other as yet unexplained, these accidents seem rather to be on the increase than otherwise. Few muscles in the extremities escape, nor are the muscles of the trunk exempt.

Certain circumstances influencing the tendency to rupture. Certain circumstances seem to influence 
the tendency to rupture, and among them may be enumerated-

1. The season of the year.

2. The temperature of the air.

3. Want of training or fitness.

4. Age and physique of the individual.

5. The kind of game or exercise.

It has often been observed and remarked upon that at the universities and public schools there are more athletic accidents during the autumn or October term than at any other time or season. The reason probably is that during the long vacation athletes have been employed in carrying out forms of exercise different from those of set athletics. When they return to school or college, without having had sufficient or gradual training, they plunge at once into strenuous games of football, running, and rowing. It is therefore not surprising that their tendons are ruptured and their ligaments torn.

The temperature of the air undoubtedly influences the muscles. Modern physiology teaches that while cold retards muscular contraction, warmth accelerates it; cold renders muscle tissue more inelastic, and inelastic muscles are more prone to laceration. The winter months, the cold seasons, assisted often by a want of proper clothing, bring many to seek the assistance of the surgeon.

Want of training is a self-evident cause. Tendons must be made accustomed to the pull of their attached muscles if they are to meet with their attacks successfully. Unless the muscles are gradually trained to the requirements of the individual, they will, indeed, are forced to yield under 


\section{INJURIES TO MUSCLES AND TENDONS}

some unexpected and supreme effort. And this leads to the following statement, that even in the case of a thoroughly trained athlete, whose muscles are properly tuned, so to speak, it would appear that his muscle is torn not entirely by its own contraction, but rather by the contraction of the antagonistic muscles taking place before its own act of contraction is completed.

Age influences in a measure.- The younger the athlete, the more supple are his tendons, and perhaps within certain limits the less powerful is the pulling force of the muscular fibres proportionately to the tendinous. Men of good physique are more liable than weaklings.

The kind of game affects the proportion of injuries; thus football and rowing are more productive than sprinting or long-distance running. Rugby football accounts for more accidents than Association, though generally they are of a less severe type. 


\section{CHAPTER VIII}

\section{INJURIES TO SPECIAL MUSCLES AND TENDONS}

In the preceding chapter certain general outlines were given as to the management of the lesser injuries to muscles and tendons, such as overstretching, hernia, and displacement, where, on the whole, the symptoms are never severe and the disablement not particularly serious.

In this chapter it is proposed to deal more in detail with the ruptures of muscles and tendons, since they often lead not only to considerable crippling at the time, but also, if mismanaged or improperly treated, to permanent loss of power.

As has been already pointed out, almost any long muscle or its tendon may be ruptured, and as it would be tedious to treat of each individually, only certain of the most important will be considered. No reference will be made to lacerations produced by external injury, or the spasms of tetanus, or during the throes of parturition ; allusion will therefore only be made to the rupture of muscles by their own forcible contraction during ordinary or violent athletic movements. The principles of treatment are the same for all, whatever the cause.

The muscles of the trunk are sometimes ruptured when direct violence is applied to them, especially 
when contracted; they may also be ruptured by their own forcible contractions.

The abdominal rectus is stated to have been torn in a patient enfeebled by enteric fever by simply turning in bed or in attempting to rise from the recumbent position. Cavalry soldiers have been known to tear the muscle in mounting on horseback, and rowing men from missing a stroke in pulling.

The chief symptoms are a sudden stablike pain below the umbilicus (for rupture always is below that point) and inability to straighten the body; a swelling is present, often of extensive proportions, transverse to the line of the body and to one side of the middle line. Usually there is no sulcus to be felt, nor ecchymosis to be seen at first. The swelling has been mistaken for umbilical hernia and cold abscess.

Treatment consists, in a mild case, or one of partial tearing, in flexing the body so that the chest approaches the pelvis, and maintaining this position in bed for one or two days. Plaster strapping is very useful (vide Fig. 74), and may be applied from the chest to pelvis anteriorly in strips or in one broad piece of strapping. When the rupture is complete and the hæmorrhage extensive, an open operation with suture of the muscle and its sheath is strongly advocated. Much time is saved, and the probability of an abdominal hernia prevented.

The oblique muscles of the abdomen are often partially torn by awkward twists or wrenching of the body. The pain is often referred to the attachments in the neighbourhood of the twelfth rib, but 
occasionally also along the crest of the ilium. Tenderness on pressure will localise the exact seat of mischief. Swelling at first may be inconsiderable, while later the signs of bruising (play of colours) may be well

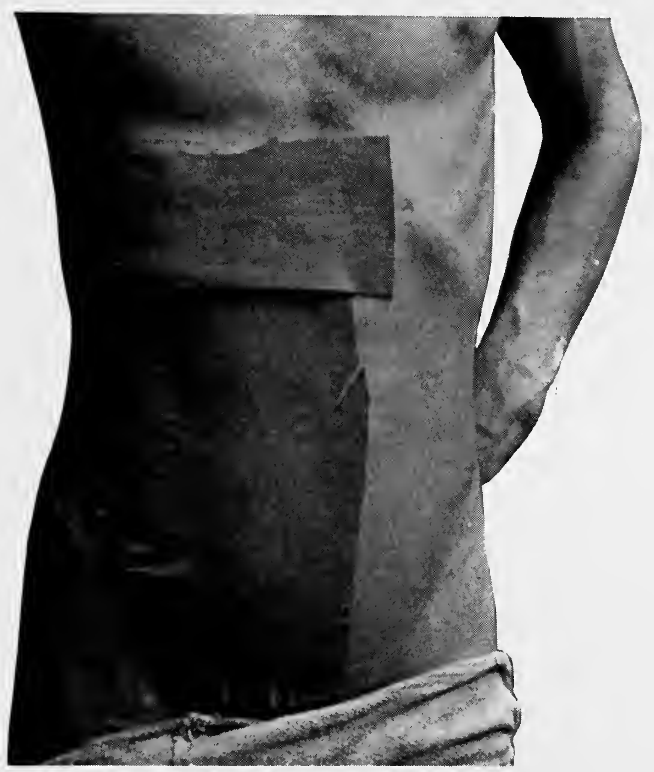

Fig. 74. Rupture of right rectus muscle of the abdomen with strapping applied of a single piece of wash-leather plaster. The strapping should reach from the upper thigh to the margins of the ribs, and be fixed above by a cross piece, and made to extend across the middle line of body. The transverse slip of plaster should be laid on firmly and not loosely as here shown.

marked. The affection known as 'bowler's side' is the result of the tearing of some fibres of one or both oblique muscles, and results from fast bowling on a wet and slippery wicket.

The most suitable treatment is to strap as for a broken rib, from the spine around the injured side 
across the middle line in front to the other side, commencing about four inches above the last rib, and extending well below the iliac crest (vide Fig. $75 \mathrm{~B}$ ), and then allowing the patient to go about as usual. Cure will usually result in from twelve to fourteen days.

The ilio-psoas muscle is rarely completely torn, though partial tearing is not an uncommon occurrence in beginners at rowing. - It results from an awkward swing of the body in the leg drive from the stretcher, and is more often produced with a fixed than sliding seat. There is pain in straightening the body, felt in the iliac fossa and upper thigh, and there may be considerable ecchymosis immediately below Poupart's ligament. The treatment consists of rest for a few days from the particular movements involved. No local application seems of much service. The ilio-psoas tendon has been ruptured in the thigh in a boy who attempted to lift a heavy basket; the injury was followed by extensive swelling.

The sacro-lumbalis may be partially dislocated or torn. Replacement of displaced fibres under an anæsthetic, and subsequent plaster strapping, is usually sufficient. While there is always tenderness over the seat of the rupture, the swelling seldom shows externally, though doubtless effusion takes place between the deep muscular planes.

In the upper extremity, the pectorals, deltoid, biceps (both long and short heads), are the most commonly torn; very rarely fibres of the triceps and muscles of the fore-arm. These ruptures result generally from indirect violence.

The pectorals have been torn in violent efforts 


\section{MUSCLES AND TENDONS}

to prevent a fall by catching at a suspended rope. The symptoms are pain on movement, tenderness on pressure, severe ecchymosis over the side

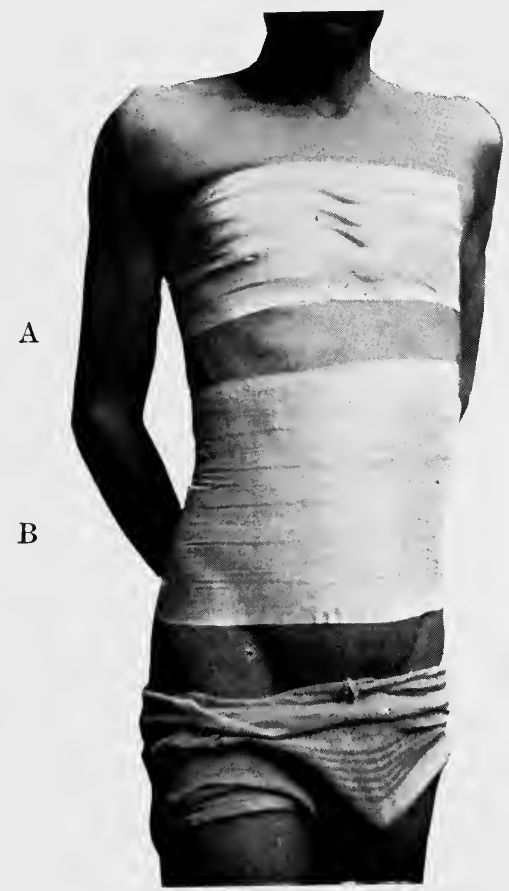

FIG. 75. A. Strapping applied for sprains of the intercostal muscles and side. The plaster should encircle at least two-thirds of the circumference of the body and be laid on from above downwards during expiration.

B is for sprain or rupture of the oblique muscles of the abdomen'bowler's side'. The plaster should extend from the lower thorax to below the crest of the ilium.

of the chest, and complete inability to use the shoulder.

Treatment by strapping the arm to the side with the fore-arm in a sling-massage often useful later.

' Cricket shoulder' is a strain produced in throwing 
a ball, and is usually caused by the rupture of some fibres of the deltoid near to its insertion, or to the overstretching or partial laceration of the sheath of the pectoralis major, where it encloses the long head of the biceps in the bicipital groove. It has been reported that even the head of the biceps has been displaced in the effort of throwing a cricket ball. Since the deltoid is very prone to atrophy and stiffness whenever it is injured, it should never be fixed in a position of rest; on the contrary, it is wise to strap the shoulder-joint with adhesive plaster and allow the patient to begin the ordinary movements early in the treatment. The same may be said in respect to the treatment of the long head of the biceps, for, if left in repose, adhesions are liable to be formed and extend even into the joint itself, leading to permanent stiffness. In all cases it is wise to support the injury by accurate strappings. The limb may be rubbed over the plaster. Active movements should be encouraged from the time the strapping is applied. When such an injury has been neglected, and the deltoid has borne the brunt of the injury, it becomes imperative to develop the powers of that muscle by special exercises, so that its fibres are made to contract. Graduated weights and pulleys are sometimes needful in aggravated cases.

When the biceps tendon is chiefly affected, the adhesions may be loosened and broken down under an anæsthetic preliminary to other treatment.

The biceps flexor cubiti is often ruptured by its own forcible contraction, and may suffer in one or other head or in the belly of the muscle after its two 
parts have united. The most common injury, and perhaps the most troublesome to deal with, is separation of the long head from its scapular origin. No method of treatment by posture seems satisfactory, for the end of the tendon is pulled away from its attachment in the glenoid cavity. Theoretically, an open operation would seem the most suitable, but it involves opening the joint, so that it is doubtful whether it is worth the while or is really practicable. The method generally adopted is that of flexing fully both the shoulder- and elbow-joints, strapping the arm across the chest, and maintaining it in this position for at least a month. The writer has met with only two such cases in his whole experience. The first he treated after the manner just described, and obtained but a moderate result. The second he met with five months after the injury, and although in this case there had been no attempt to retain the arm in the flexed position for longer than a week, the result was, to say the least, as good if not better than in the former. This has led him to the conclusion that such a case might be better treated as other cases of rupture are, by strapping and early movements, avoiding for a time anything that will unduly impinge on the injured tendon itself. The diagnosis is very simple, as the individual is unable to perform the usual movements of the muscle. The treatment of rupture through the belly of the muscle differs according to the extent of the injury. When the tear is only partial strapping alone may be sufficient, but when there is a complete rupture an open operation with accurate suturing is perhaps best. 
Rupture of the muscles and tendons of the fore-arm. A few cases of partial tears in the muscles and rupture of the tendons in the fore-arm have been met with, and some have been recorded. The accident is, however, comparatively rare. They may be treated by the methods ordinarily advocated in this volume for injuries of a similar nature.

In the lower extremity many of the muscles and tendons are liable to rupture, thus one or other muscle or tendon in the extensor and adductor groups of the thigh, as well as the hamstrings, suffer as the result of violent athletic exercises, while rupture of the calf muscles is no uncommon occurrence.

The rectus femoris may tear in any part of its muscular course, or its direct head may pull off the epiphysis for the anterior inferior spinous process of the ilium in persons under the age of eighteen. The accompanying photograph (Fig. 76) shows a typical example of rupture through the body of the muscle. It happened to a gentleman in endeavouring to keep his balance while skating. This form of injury, as it sometimes occurs in a sudden effort to run while fielding at cricket, has been called 'cricket thigh'. Fig. 77 is the photograph of the left thigh of a hospital patient, showing a rupture of the rectus muscle in its lower third. It was the result of a slip while playing at football. It caused very little inconvenience at the time. Now, some months later, the patient complains only of slight weakness in the limb, and loss of pace in running.

Two cases of separation of the epiphysis for the anterior inferior spine of the ilium have been 
reported. ${ }^{1}$ They occurred in undergraduates who injured themselves while starting in a sprinting race when the body was suddenly made to assume the erect

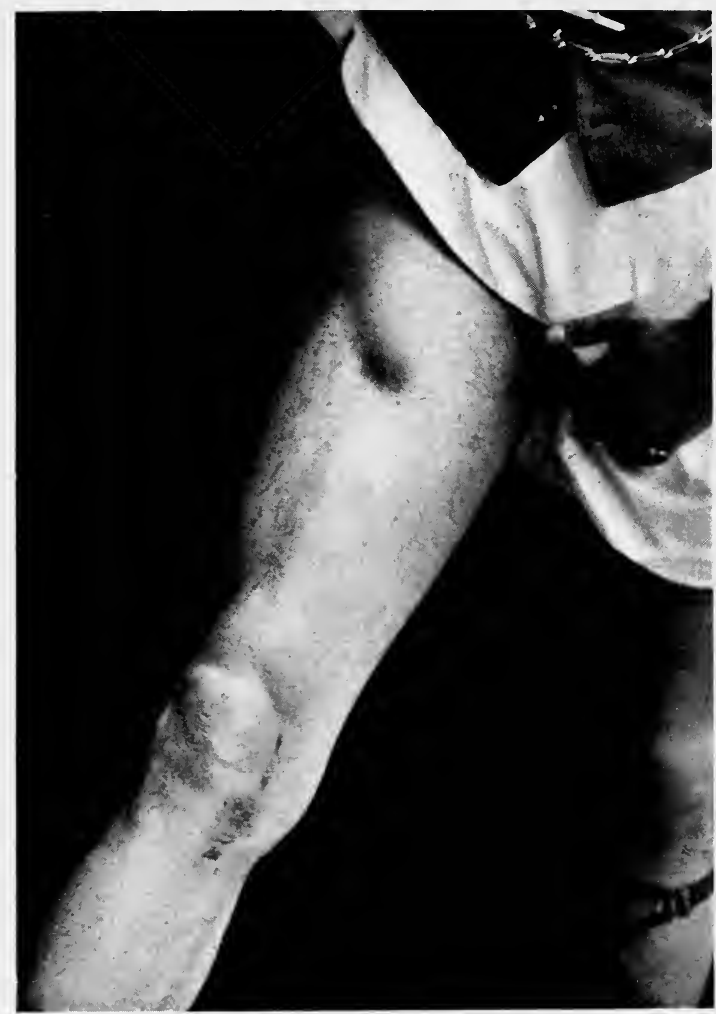

FIG. 76. Rupture of rectus femoris muscle, done while skating. Patient subsequently displaced the internal semilunar cartilage of his knee, which was removed by the author. The scar on the inner surface of the joint represents the line of incision.

position after being in a stooping one. The details as to the precise mechanism of the injury and its subse-

1 'Separation of the epiphysis for the anterior inferior spinous process of the ilium through enforced muscular action.' By R. H. Anglin Whitelocke, Lancet, 1893, vol. ii, p. 1302. 
quent treatment are there fully gone into. It is sufficient to state that they were at that time treated as a fracture would be, with a plaster-of-Paris spicabandage.

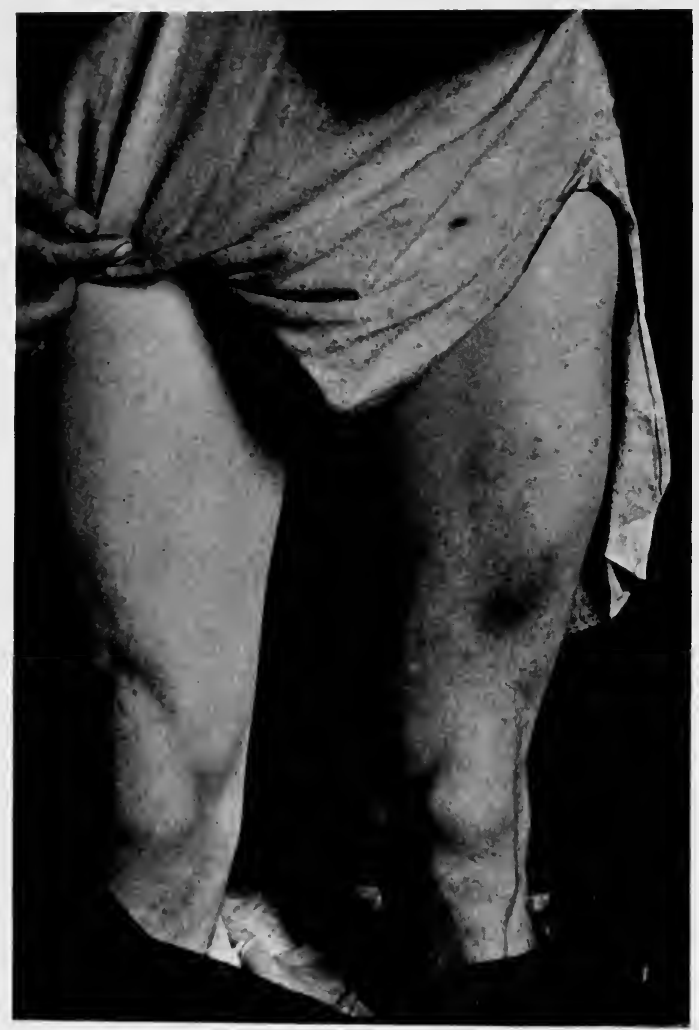

FIG. 77. Photograph of rupture of rectus femoris muscle in its lower third (vide text).

The symptoms of rupture in the rectus femoris are surprisingly slight. There is little pain; often the feeling of snapping or tearing is experienced, followed by a weakness in the limb. Swelling may be imme- 
diate and extensive, and even mask the existence of the usual sulcus when viewed for the first time; palpation will, however, very readily disclose its presence.

Treatment depends upon circumstances. The direct head, with its detached epiphysis, may be treated by temporary fixation by strapping and allowing the patient to walk about, or by a plasterof-paris bandage and crutches. The former plan will probably, judging by analogy, yield a more satisfactory result. Where there is complete rupture through the belly of the muscle, especially where there is a large hæmatoma and no serious contraindication as to general health, the open operation is the most suitable. When the rupture is only partial, strapping the thigh with broad strips of Seabury \& Johnson's perforated rubber plaster so as almost to encircle the limb and draw the muscles together, is quite efficacious. The plaster (vide Fig. 78) should be applied in strips at least three inches wide. It should never be allowed to encircle the thigh completely. A good plan is to leave a gap of about an inch between its ends. This gap allows enough play in the muscles of the thigh during walking and other exercises, and unless it is left, when the muscles enlarge by contraction, they will either burst asunder the application, or tear their own fibres at the point of greatest constriction. The plaster, properly applied, gives ample support, and enables the individual to walk about at once, and in the milder cases he may even resume his athletic exercises in the space of a few days. Another result. of the injury is met by this method of strapping. wHITELOCKE 
The skin and fasciæ are supported by the firm, more or less resilient plaster, so that the extravasated material is compressed. As the patient walks, the muscles of the thigh, owing to their contraction, become alternately larger and smaller, so that a to-

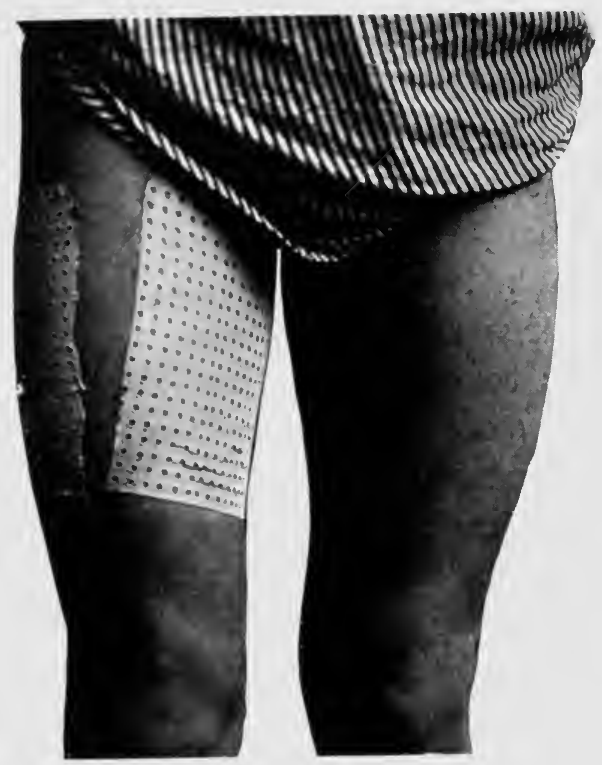

Fig. 78. Photograph of thighs of patient to show on the right side author's method of strapping for rupture of fibres of the hamstring muscles. The plaster is not allowed to meet, or to completely encirele the limb. A space is left to allow of muscular expansion during exercise. For rupture of the adductors or extensors of the thigh the separated ends of the plaster are placed externally.

and-fro movement is exerted on the materials that are extravasated between them and the overlying skin; by this churning process the extravasation becomes dispersed up and down the thigh under the strapping, and is driven by the elastic compression 
in the direction of the venous flow, and hurried onward into the general circulation. The effect produced on the local circulation is not unlike that of friction massage.

The advantages of strapping when applied to ruptured muscles may be summarized as follows. When it is evenly and smoothly applied, further extravasation becomes impossible; the muscles are supported so that further tearing is prevented; it becomes safe to allow the individual to walk or to otherwise exercise his muscles, and the exercise will not only prevent weakness and muscle atrophy, but it actually disperses and drives onwards all local extravasated material into the general blood-stream. Healthy local nutrition is ensured with much certainty, as the general health of the patient will be bettered if he is allowed to go about rather than live in the house during convalescence. This is the whole rationale for the use of strapping in the treatment of ruptured muscles. The fibres that are torn seldom unite completely, and this seems a matter of little consequence, for other fibres develop to take their place, or neighbouring muscles enlarge to make up for any deficiency. The limb should be carefully shaved, washed over with a spirituous lotion, and sponged dry before the rubber plaster is applied. Seabury's Rubber Plaster is sufficiently adhesive, and as it contains rubber does not readily slip. The perforations allow of a measure of evaporation, yet even this will not prevent a modicum of eczematous irritation occurring in the skins of certain individuals. The irritation when it does occur acts therapeutically as a somewhat mild counter-irritant, and perhaps in 
the end does good and compensates the individual for his discomfort. As soon as the plaster becomes loose by reason of the absorption of the subjacent extravasation, it must be removed and re-applied; this may be necessary on the second or third day when the extravasation has been copious in the first instance.

Rupture of the quadriceps extensor tendon is a troublesome injury, and results, from a sudden effort to regain the balance. Whether the patella fractures, or this tendon or the ligamentum patellæ tears, usually depends upon the amount of flexion in the limb at the time the strain takes place. The diagnosis is easy: the gap in the tendon with loss of power in the limb are quite sufficient to distinguish the condition.

The treatment is of the greatest importance. In slight cases, where there is only partial rupture some distance from the knee and without much extravasation, the method of strapping mentioned above will suffice, providing also that the limb is kept in the extended position for about ten days. The leverage at the knee is so great that it would be unwise to allow flexion before some definite healing had had time to take place. When the rupture takes place near the patella the matter is most serious, and it should be treated just as if the patella itself had been fractured. The joint is sure to be opened into and to be full of blood. When there are no definite contra-indications such as organic disease or the patient's refusal to. allow operation, the joint should be immediately opened, the clots turned out, and the fibres of the tendon sutured as accurately as possible with catgut. 
This operation has been performed on several occasions, always with success, and the view is strongly held that if the surgeon has perfect confidence in his operative technique, he will obtain better results by the open method than by any other. The patient will be able to walk in four weeks and to do practically anything in six weeks, providing always that his splints are left off not later than a fortnight, or soon after complete healing has taken place in the skin wound, and that early movements are practised. The details of the operation will be described more fully when dealing with rupture of the patella ligament.

The hamstrings are seldom completely torn across, though partial tears are very common in broadjumpers, sprinters, and football players. The two most common sites are close to the common origin from the tuberosity of the ischium and about the centre of the back of the thigh. The biceps, semitendinosus, and semi-membranosus may all suffer. The tearing results from an awkward landing in the broad-jump by the individual endeavouring to prevent falling backwards after the jump. Sprinters unaccustomed to run in shoes with spikes and without heels frequently tear portions of the hamstring muscles before they get into proper training. A rarer injury is one found in the neighbourhood of the insertions of the semi-tendinosus and gracilis to the inner surface of the tibia below the knee, caused by an overstretching in certain lateral movements of the leg as in 'side-kicking' at Association football. It gives rise to pain, swelling, and impaired movements. A depression may be felt in the hamstring tendons close to the ischial tuberosity, but 
in the majority of cases the diagnosis is made from the history of 'something going' during running or jumping, and although at first there are few signs of extravasation, the usual play of colours of an old bruise will develop later. The treatment consists in raising the heel of the boot, strapping as for other muscle-tears in the thigh, and allowing the patient to walk about from the very first. As a rule the cure is effected in about fifteendays. Recurrences, however, are not uncommon.

In the adductor group, the adductor longus et brevis, as well as the pectineus, are torn, from muscular efforts pure and simple.

The adductor longus is so frequently injured in those engaged in horse-riding - as cavalry soldiers and huntsmen-that the name of 'rider's sprain' is now almost universally adopted in surgical literature to denote this particular affection. The muscle may be torn close to the pelvic attachment or at its lower end; when in the former situation, which is the more usual, the nerve of supply is often damaged and atrophy follows. There is always extensive bleeding, and the swelling passes up to, or even above, Poupart's ligament, and down into the perineum and thigh. The pain is sometimes slight and out of all proportion to the severity of the injury, but not uncommonly there is a severe stabbing pain referred to the region of the anus. The loss of adductor power is considerable, and the tenderness on pressure over the whole region is marked. The diagnosis is simple; the history of something 'snapping ' while riding a swerving horse, or in mounting a restive animal, inability or difficulty in performing 
adduction, followed by severe extravasation and the presence of a gap in the course of the muscle, make it certain.

The treatment in recent cases is directed towards preventing and removing the extravasation. Much time will be lost if the old régime of using cold lotions is adopted. Either elastic pressure by means of cotton-wool and a bandage, or firm rubber-strapping, serves the purpose better. If a definite hæmatoma forms, and there is no reason against it, an incision should be made, the clots removed, and the muscle itself either sutured or excised. The loss of the muscle is surprisingly little felt. Cases are on record where it has been removed late in the case because the proximal end formed a painful swelling, troublesome to the rider, and in a few which were followed by ossification. The other adductor muscles develop and take on its functions. In mild, or incomplete cases the part should be strapped with plaster, and the individual allowed to walk about, or even ride a quiet steady hack after a few days. Some of the writer's most successful cases have been treated in this way. When the adductor brevis or pectineus is torn it must be treated on similar lines.

The adductor magnus tendon, at its insertion into the adductor tubercle, is sometimes strained in injuries involving the inner side of the knee. Tenderness is occasionally experienced extending along the muscle high up into the thigh when the internal lateral ligament of the knee has been wrenched or torn in sprains of the knee.

The ligamentum patellæ may be torn either from its lower attachment to the tubercle of the tibia or 
from the patella above. In each case it is usual for a small fragment of bone to be detached with it. In rare cases it snaps across near its middle. It may be incompletely torn. The diagnosis is by no means difficult, for the patella is usually displaced and drawn upwards above the knee. When very small fragments of bone are detached, nothing but the $\mathrm{X}$-rays will make the diagnosis certain. It is usual to regard this injury as being, of as much serious consequence as a fracture of the patella (vide also Schlatter's disease, Chapter V).

As to treatment, posture and fixation is a very uncertain method, and as the cavity of the knee-joint is always torn into, as is the case in complete fractures of the patella, the joint is filled with blood. Whenever there is no insuperable objection to an operation it is best to open the joint and to suture the ligament accurately with catgut. The writer has used this plan in four recent cases with the greatest satisfaction. All except one could walk freely and bend the knee well beyond the right ankle in the space of five weeks. The exception, a timid boy, did not obtain full movements until ten weeks after the operation, but this was chiefly because he would not exercise the limb when left to himself. The technique is the same as for suture of fractured patella, an operation which the writer now invariably performs, by using catgut instead of wire. The accompanying Figs. 79 and 80 illustrate a case in which both the ligaments were torn at the same time in a man who, having slipped, tried to save himself from falling backwards. His case was subsequently treated elsewhere for months by posture and splints, with the result that there was 
no union on either side. He has, however, been able to walk with the aid of the somewhat cumbrous appliance, depicted in Fig. 81. The case was not considered suitable for operation owing to his age and alcoholic habits.

'Lawn-tennis leg' has been variously attributed to rupture of the plantaris tendon, to rupture of

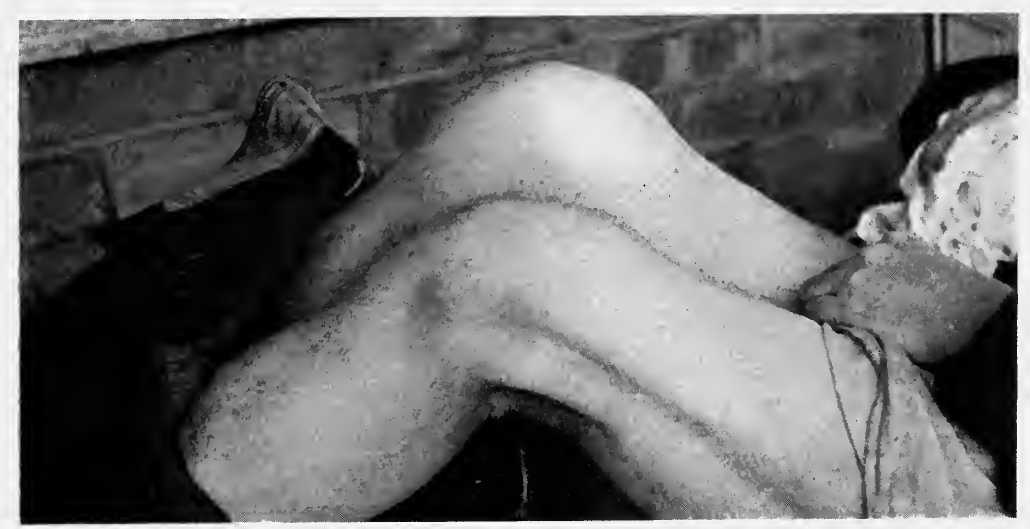

Fig. 79. Old case of double rupture of the patellar ligaments. The patient had met with the accident many years before, from trying to save himself from falling backwards after slipping in the street. He was able to walk with the aid of a hinged apparatus worn on each knee. The characteristic flexion of both joints as the result of loss of power in the extensors as well as the subsequent contraction of the hamstrings is well shown. Patient refused operation. Mr. R. H. Sankey kindly procured the patient, and made the illustrations for the author.

fibres of the soleus, and even to an injury to the deep veins situated between the muscles of the calf. That sometimes the symptoms are due to the rupture of one muscle, and at others to another, there seems little doubt. A few cases have been met with in which the symptoms could best be attributed to a separation of some of the fibres of the soleus muscle 
from its attachment to the oblique line of the tibia. The symptoms are sudden pain, often described as if the individual had been struck by a stone thrown at him, or cut with the lash of a whip, followed by an inability to walk, and later by swelling and tender-

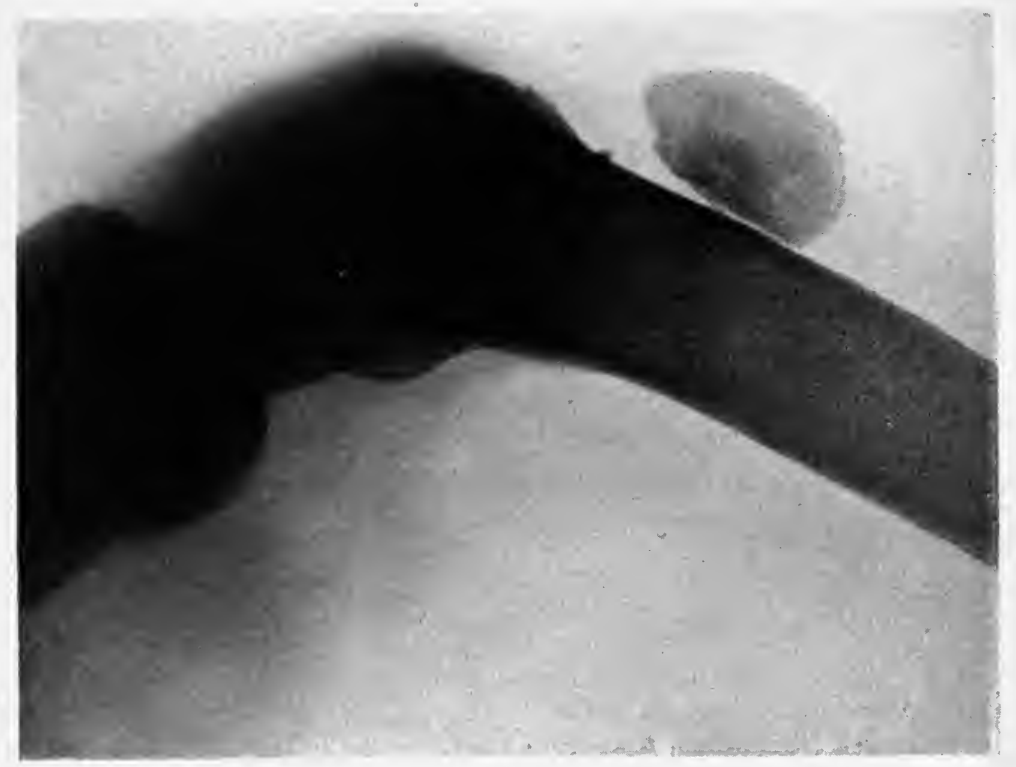

Fiti. 80. Radiogram of case of ruptured patellar ligament of long standing to show the displacement of the patella upwards from the front of the joint to the anterior surface of the femur. Taken from the same case as is described in the text.

ness of the calf with discoloration of the skin near the ankle. The diagnosis is made from the history of the case taken together with the symptoms.

The most suitable treatment is to strap the leg from two inches above the ankle up to a level two or three inches below the knee, by means of stout rubber plaster, and then to allow the patient to 
walk about, making a point of keeping his heel flat upon the ground all the time. A flat, low-heeled boot is best; even running may be permitted in a few days with this line of treatment; active exercises should not, however, be permitted until all or most

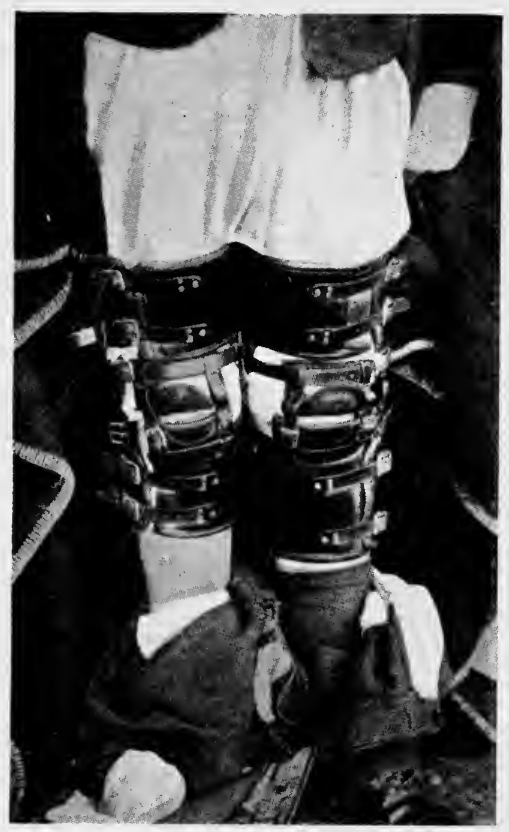

FIG. 81. To show the apparatus worn for many years by a patient, the subject of rupture of the patellar ligament on both sides. He was able to walk with tolerable comfort.

of the swelling has disappeared. The subsidence or not of the swelling is the best guide as to the commencement of active exercises. The strapping should be removed and re-applied as often as it becomes loosened by the absorption of the effused blood beneath it. The prognosis is invariably good, and 
the patient should be free from all symptoms in from a fortnight to three weeks.

When, however, the injury is due to tearing of the fibres of the soleus, recurrences may be met with, though they are not common.

The tendo-Achillis is often ruptured from one to one-and-a-half inches from its attachment to the os calcis as the result of sudden violent strain. The symptoms are so slight that many cases are on record in which the individual walked about afterwards for even considerable distances with but little pain and inconvenience. A feeling of weakness in the ankle, whenever she got on to her toes, was all that was complained of by a young lady of eighteen, who ruptured this tendon last year in jumping out of a punt. When she first presented herself for inspection she limped slightly into the room and seemed to have little or no pain. On making an examination, eighteen hours after the accident, there was found considerable ecchymosis over the region of the heel, and a distinct gap could be made out at the narrowest part of the tendon. There was tenderness on pressure over all the muscles of the calf. Whenever the ankle was bent, the feeling of discomfort was increased and the gap in the tendon became more evident. The case was treated in the usual textbook fashion, by means of a laced shoe, to the heel of which an elastic apparatus was fixed. The elastic band was then passed upwards to be attached to a broad leather strap which fitted accurately the middle of the thigh and was so arranged that the heel was drawn towards the knee and the toes pointed. This allowed of complete relaxation of the muscles of the calf. The 


\section{MUSCLES AND TENDONS}

apparatus was worn for fourteen days and then removed. After this, gentle muscular exercise was allowed while she lay on the sofa, but the full weight of the body was not permitted until twenty-eight days had passed. When she first began to walk she

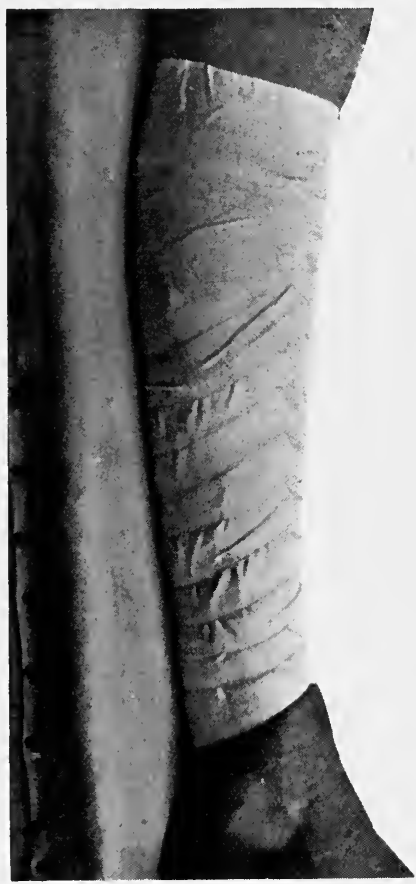

Fig. 82 represents a leg that has been strapped for tearing of some of the fibres of the soleus muscle. The strapping has been too tightly applied below. To prevent constriction, the lower strap should be applied over a piece of folded lint or proper padding.

complained of a feeling of insecurity, but this passed off in two or three days, when she expressed herself as being not only able to walk but to run.

John Hunter ${ }^{1}$ is said to have ruptured his tendoAchillis. The accident occurred when he was aged

$$
1 \text { Palmer, Life, p. } 34 .
$$


thirty-nine, while dancing. ' $\mathrm{He}$ did not confine himself to bed, but continued to walk during the cure.' He kept the heel raised, and compressed the muscle gently with a roller. $\mathrm{He}$ is said, however, to have experienced ossification of his tendon. It is interesting here to note that so long ago as Hunter's time it was considered sound practice to allow a patient to walk about with a torn tendon, providing, however, that it was strapped and supported. The fact that ossification of the tendon supervened had nothing to do with the ambulatory method of the treatment, but was probably simply another example of the unavoidable sequelæ of a sprain, to which reference has already been made. Do what the surgeon may, in certain cases of articular sprain osteo-arthritis will supervene, and in muscular strains, myositis ossificans. These untoward effects are so far regarded as non-preventable. Every surgeon is aware of the ready way in which tendons unite after tenotomy, and many will have seen old cases of rupture through tendons, where very fair union has taken place, in animals and persons who had received but scanty treatment, and where walking and other exercises have been permitted, or at any rate been indulged in. The writer has recently made dissections of two such specimens, one in a horse and the other of the tendo-Achillis of a dissecting-room subject. The blood-clot which fills the intervening space in the tendon-sheath readily organizes, and will do so whether the tendons are put at rest or whether they are exercised or moved from the beginning. Prolonged fixation by apparatus is, as a rule, quite unnecessary. If non-union should occur, and the 


\section{MUSCLES AND TENDONS}

disability is great, an open operation, with suturing the ends of the tendon, can be at any time performed. It is a safe and comparatively simple matter. There are many instances on record of the successful suturing of tendons; indeed, such is an everyday occurrence.

The treatment consists in strapping the whole length of the tendon firmly to the bones of the leg by encircling strips of plaster, and allowing the individual to walk about with a somewhat raised heel as soon as the tendency to swelling has ceased. It has proved a matter of considerable interest to find that the system of treatment so consistently advocated in this volume is precisely similar to that taught and practised by John Hunter so many years ago. 


\section{CHAPTER IX}

\section{MASSAGE, MOVEMENT, AND EXERCISES IN THE TREATMENT OF SPRAINS AND BRUISES}

From time immemorial rubbings and movements have been used for the relief of stiffness and pain in joints. The practice, like many other things, seems to have originated in the East. Formerly much in vogue in this country, for a time it almost dropped out, only to be revived in recent times with renewed vigour and unabating popularity. The system is the chief asset of the itinerant quack and everwise bone-setter ; and, like most good things, it is liable to be abused.

Massage and movements, when employed in the cure of disease, are surgical procedures, and as such should only be used under the supervision and guidance of persons trained in surgical methods. Unless some such limitations are imposed, harm will result. To take a single example, what could be more baneful than the daily rubbings of a joint swollen and full as the result of early tubercular infection?

Before proceeding further, it may be useful to discuss the so-called 'physiology of Massage'. The various movements of massage undoubtedly improve the nutrition of an injured part by primarily increasing its blood-supply. By gentle friction-massage the local blood-vessels become dilated, and fresh oxy- 
genated blood is brought; it is easy to satisfy one as to this, for not only does the skin of a joint formerly cold and pale become reddened, but it is warmer to the touch. By experiment, Drs. Brunton and Tunnicliffe demonstrated that not only does more blood pass through the muscles in a given time while they are being massaged, but that the increased flow lasts for some time after. Doubtless all the tissues in the neighbourhood of the injury partake of the increased blood-supply and reap the advantage.

The kneading and percussion movements act mechanically in breaking up all coagulated material, such as blood-clot and lymph, found amongst the muscles and tendons and other tissues around an injured joint ; and by dispersing the fragments over a larger absorption area and into more healthy parts, aid most materially in the absorption of the effete material by the neighbouring lymphatics and veins. By stimulating the nerve-endings friction-massage would seem to produce, reflexly, a mechanical dilatation of the vessels of the part. In that way it increases the blood-flow through the muscles and other tissues. It has a decided influence in diminishing pain, for most persons will have experienced the soothing effect it has if applied gently to a painful spot. This effect is probably due to some reflex act, for the relief follows on too quickly to be explained by any direct effect it may produce upon the nerveendings themselves. Some of us may have experienced the almost immediate relief of neuralgic pain as the result of taking a hot cup of tea or a dose of caffein. The action in this latter instance is doubtless due to an alteration in the circulation of the 


\section{MASSAGE, MOVEMENT, AND EXERCISES}

nerve-endings following upon the ingestion of a rapidly diffusable stimulant. Whatever the true explanation, it is a fact that massage readily relieves pain when carefully applied to a strain. Musclewaste is probably in a measure prevented, partly by a direct stimulation of the nerves of supply and partly by indirectly increasing the local blood-supply.

It promotes the absorption of effused materials, whether they are enclosed in the synovial cavities or extravasated diffusely into the tissues generally.

Limitations of massage. The immediate use of massage in sprains, either with or without passive movement, which it should at all times precede, is a practice much in vogue at the present time. By some surgeons it is adopted from the very beginning of the treatment and kept up until even long after recovery is complete. While in no way wishing to underestimate the value of massage, especially in long-standing cases and in the later stages of ordinary cases, the view is strongly maintained that its immediate use, in the large majority of cases, is not only not necessary but may be even harmful. This statement requires some proof.

It is an everyday experience that cases such as those in which a cutting operation has been performed, or in which, from the presence of some abrasion or wound of the skin, local massage was impracticable, get on just as well and recover quite as quickly in the end as those in which rubbing was applied from the very first.

Again, it is an undoubted fact, that, given a joint with commencing effusion, rubbing, however gently performed, will at once increase the extravasation, 
and if persevered in for any length of time will lead to marked increase of tension and even pain.

Nor is it difficult to understand that a hæmorrhage resulting, say, from laceration of a large muscle like the crureus or rectus abdominis will be increased by manual manipulation, and this is just what actually does take place when immediate massage is applied to any large collection of blood.

Massage should not be used until the effusion in a joint has ceased to increase, and never till all risk of encouraging and promoting hæmorrhage has passed.

It must be applied with extreme caution in all cases in which there is any likelihood of constitutional disease appearing as a complication. Such diseases as gout, tuberculosis, hæmophilia, acute or subacute rheumatism, are especially likely to be made worse by such treatment. The main indications are found in

1. All cases of strain or sprain without swelling.

2. All articular sprains, after the effusion has disappeared or has nearly disappeared either spontaneously or after treatment with elastic pressure.

3. Muscular sprains, when all risks of increasing the swelling has passed.

4. All long-standing, chronic cases, where pain, stiffness, swelling, matting together of the tissues, or atrophy, exist.

It is useful for muscular as well as articular sprains.

In sprains complicated by fracture, separation of epiphyses, and displaced interarticular cartilages, it must be very cautiously employed.

If the movements increase the pain rather than 


\section{MASSAGE, MOVEMENT, AND EXERCISES}

diminish it, they should be withheld, at any rate for a time. It is unusual for pain to be increased in ordinary cases, so that when massage is uncomfortable and badly borne some complication may be suspected.

Passive movements are of more limited application, should be employed as an assistant to massage, and always follow, never precede it. The movements are mechanical in character and are of service chiefly in restoring to their natural positions tissues that are torn and displaced, in stretching or breaking down recent adhesions, and in preventing the formation of new adhesions after old ones have been forcibly torn asunder in the treatment of stiff and ankylosed joints.

It has been stated that they assist in the absorption of extravasated material, but this is doubtful ; they certainly have no influence in preventing muscular atrophy. The contra-indications are precisely those of early massage.

In sprains with fracture, when there is a probability that active voluntary movements would produce a separation of the fragments, passive movements are preferable to active. Even in this class of cases they must be employed with much caution, and whenever it is convenient the surgeon himself should carry out the manipulations.

Active or voluntary movements act physiologically and are of the greatest service. Their application is almost universal, and, with certain simple and rare limitations, may be employed at all stages and in all conditions of sprain whether articular or muscular.

A voluntary movement implies the passage of 
a voluntary nervous impulse such as controls the whole process of nutrition in the tissues of the body. The several indications that require surgical assistance, such as the presence of extravasated material within or around a joint, the formation of adhesions, stiffness as a consequence of matting of the tissues together, and muscular atrophy, may all be removed by muscular movements.

All that massage and passive movements are said to be capable of effecting may be more speedily and efficiently attained by means of elastic pressure combined with voluntary movements executed either simply or against resistance.

Elastic pressure, as explained when dealing with the treatment of effusion in Chapter II, is the surest and safest means which we possess for attaining rapid and complete absorption of fluids. It has all the advantages claimed for massage in early treatment, without any of its disadvantages, and when combined with active movements, may be described for all practical purposes as ideal. For by mechanically and rapidly removing the effusions and passing them on again into the general circulation, tension is diminished, pain relieved, and adhesions prevented. Taken conjointly with muscular movements, which alone can prevent muscular atrophy, it meets every ordinary desideratum.

In urging early voluntary movements it must be understood that it is not suggested that all the muscles of an injured part should be exercised, or that they should be vigorously moved; for, on the contrary, it is of the greatest importance that the movements at first should be of the gentlest possible 


\section{IIASSAGE, MOVEMENT, AND EXERCISES}

kind, and only just sufficient to ensure the passage downwards of nervous impulses. It is also important that until repair is fairly advanced, the particular muscles that have borne the brunt of the incidence of the violence should be subjected to very little or no strain, while the neighbouring muscles, tendons, and ligaments are freely exercised.

In the later stages of treatment, and especially in neglected sprains, definite muscular exercises and movements often against resistance are of the greatest possible service in restoring movement, preventing atrophy, and assisting in the regeneration of muscles already wasted.

Various gymnastic exercises, performed with or without the aid of mechanical contrivances, have been instituted for promoting the development and regeneration of muscles, and for removing stiffness and incapacity (vide Mechano-therapy, Chapter X).

Forcible movements of stiff joints under an anæsthetic. These should never be undertaken until a careful diagnosis has been made, often involving much trouble and difficulty. Unless the surgeon is prepared to bring into play his most discriminating powers, he may do as much harm as the bone-setter who, in a routine way, treats everything by movement. Movements under an anæsthetic find their chief utility in healthy joints, and they for the most part act mechanically. For the replacement of interarticular cartilages, stretching or breaking down synovial adhesions, restoring lacerated portions of a capsular ligament to their natural situations, altering the angle in which a joint may become fixed, they are of special value. 
If the joint, however, has already undergone any definite organic change in its structure, such as may result from Charcot's disease or other forms of osteoarthritis, or if the stiffness is mainly due to the taint of some such constitutional affection as tuberculosis, chronic rheumatism, gout, or pyæmia, it is quite unsuitable for any forcible movement. The conditions in which most good may be expected are those in which a joint is stiff or even painful because its action is hampered, not by any disability within the joint itself, but owing rather to the matting together of its surrounding tissues by unabsorbed extravasated material. The shoulder, as we have seen, is, of all the joints, the one which affords the best example of such a state of affairs, and it is the one from the treatment of which the surgeon is most likely to gain satisfaction.

Stated broadly, it may be said that the results of forcible movement are not as good as we may be led to expect from theoretical considerations, that they are often very disappointing, and will assuredly be even disastrous unless employed only after the strictest discrimination and selection, and with the greatest care and skill in management.

Pain and stiffness are general indications for forcible movement in a joint; but it is often a difficult matter to decide at once whether or not to recommend it. A good and serviceable plan is to carefully investigate the range of movement and to notice whether the pain is experienced when only a wide range is attempted, or whether it is felt during every movement, however slight. Thus a shoulder crippled or months, with shining surface, atrophied deltoid, 


\section{MASSAGE, MOVEMENT, AND EXERCISES}

and apparent absolute fixity, painful whenever any extensive movements are attempted, may prove, after close investigation, to be eminently suitable. When the movements, if ever so limited in range, are smooth and afford no sensation of roughness or jarring, one may be fairly confident that the trouble is outside the joint and not within it. A joint with perfectly smooth movements is rarely, if ever, disorganized or organically wrong.

So long as the stiffness is outside the capsular cavity and is mainly concerned with the structures around the joint, forcible movements may be undertaken with every prospect of complete success.

Author's method for preventing atrophy in the muscles of thigh and leg. For developing the muscles of a thigh and leg that have atrophied as the result of prolonged fixation in splints, the following manœuvre has proved of great service in many recent cases. It is of more certain value than are the movements against resistance as practised in gymnasia with the aid of pulleys and elastic apparatus. It consists, when practicable, in making the patient assume the squatting position. In this posture the hip, knee, ankle, and metatarso-phalangeal joints are all flexed, the hamstrings are relaxed, while the extensors of the thigh are fully stretched. The ligamentum patellæ is held taut all the while. During dorsiflexion of the metatarso-phalangeal joints the muscles of the calf are stretched by the fixity of the tendo-Achillis below, while the muscles of the front of the leg are also put on the stretch. At the commencement of treatment this is all that is necessary, and the squatting position may be 
assumed for two or three minutes at a time, three or four times in a day. In this way all those tissues which, while retained in the extended position were relaxed and placed at rest, are now put upon the stretch and exercised. It is surprising with what rapidity restored power begins to show itself with the assistance of this simple plan. As soon as the erect, or even the sitting position is resumed after squatting, a sense of relief is immediately felt, and a feeling as of work done is experienced. This simple device of stretching the muscles, tendons, and ligaments by squatting, brings into play nervous and other stimuli which were wanting in a limb made to assume for a prolonged period the extended position. The natural stimulation thus brought about in the muscles which for weeks, or longer, had been thrown out of use, is most beneficial. The individual experiences at once a feeling of increased warmth and renewed vigour in the limb. After the squatting has been practised for a few times, the patient should be encouraged to raise himself gently on to his toes as in attempting to rise. A little more time should be devoted to this each day, until by degrees he is able to raise himself completely to the erect posture, and to carry out the exercises of alternately squatting and rising, slowly and gradually. This should be continuously practised till the limb becomes again strong and the muscles regenerated. At first both limbs, the sound as well as the affected, should be made to execute the movements, and, as improvement takes place, the unsound limb should be made to do more and more in proportion. An improved nutrition is the first sign of progress, and this is soon succeeded 


\section{MASSAGE, MOVEMENT, AND EXERCISES}

by true regeneration and growth. The movements, when carried out naturally, and gradually and carefully increased, may be said to be ideal in their results.

These principles of graduated exercise may be adapted with equal advantage to the muscles of the upper extremity, or indeed to any muscles in the body.

The means whereby the individual is enabled to fully exercise his muscles by lifting his own body weight are not only more easily and conveniently employed, but they seem to be more rapidly efficacious than are the stock exercises by means of weights and pulleys, so often recommended.

The natural and voluntary impulses thus sent down from the brain to the muscles on both sides at the same time that muscular efforts are made, would seem to promote more readily the regeneration of muscle-tissue than does any more mechanical plan of action, such as pulling or working against resistance. With such a plan of treatment, gymnasia are unnecessary, and all that is required is a thorough understanding between the surgeon and his patient as to the simplest means by which healthy physiological movements may be executed. It may be well to remind the reader that in the execution of all muscular movements, especially when instituted for the regeneration of muscle-tissue, there should be no hurrying. It is advisable that the movements should be slow and gradual. A distinct interval of time or pause between any two muscular contractions is beneficial. During this interval fresh arterial blood flows to the contractile tissues, while with the succeeding contraction the used-up materials are made to pass onwards with the venous blood back 
into the general circulation. It is obvious that any means employed to assist in establishing a good circulation must materially benefit a damaged tissue.

As a preventive of muscle-wasting the writer knows of no method which even approaches this in its results either as to efficiency, simplicity, or rapidity.

Movements and exercises against resistance. No one will gainsay the importance of exercising weakened or stiff muscles by means of weight-lifting or other methods in gymnasia, as often, without some such means, cases go from bad to worse; but it is here contended that these systems will be less and less required as the more rational treatment of sprains becomes more and more universally adopted. A sprain properly treated in the first instance will rarely, if ever, require gymnasium treatment. 


\section{('HAP'TER X}

\section{SPECIAL IIETHODS OF TREATMENT}

Local Applications

(1) Counter Irritants

Of local applications, the following have been found useful :

Soap Liniment (Linimentum Saponis) is convenient for rubbing in almost all cases in which massage is indicated. It provides a suitable lubricant, and prevents chafing of the skin wherever friction is considerable, and may be used in acute as well as chronic cases.

Iodine Liniment (Linimentum Iodi) is occasionally useful as a counter-irritant, and is stronger than the tincture of iodine. It has the disadvantage of being rather irritating to some skins; is most useful for painting chronically inflamed joints, and in tenosynovitis.

Terebine Liniment (Linimentum terebinthini Acetici) is a rubrifacient and mild counter-irritant when rubbed in, is useful in strains of both muscles and joints where there is little or no swelling, and may also be used in joints that are chronically painful.

Vasogen-Iodine is par excellence the most useful application, for it possesses all the advantages of the liniment of iodine without its disadvantages ; and it may be used almost universally for acute as well as chronic cases, and causes little or no irritation. 


\section{SPECIAL METHODS OF TREATMENT 253}

Tincture of Iodine is of little real value ; is apt to irritate or harden the skin, and is inferior both to the liniment and vasogen-iodine, is sometimes useful in the mildest cases, and where only a few applications are required.

Scott's (mercurial) dressing is helpful in sprains complicated by tuberculosis, and in some very chronic forms of relapsing synovitis.

\section{(2) Bandages and Strapping}

Plain rubber bandages are not recommended. They are too powerful, often produce pain, are hot, allowing little or no evaporation, and if long continued or constantly worn, tend to deplete the part of its proper blood-supply, thereby lowering its nutrition, and producing muscle-wasting and enfeeblement.

Elastic webbing (of from 2 to $2 \frac{1}{2}$ inches in width) is more useful as a supporting bandage, and should be substituted for the plain rubber bandage. Such a bandage is chiefly useful as a support, either in the early convalescence or on the return to athletic exercises. It should never be worn continuously, and should at least be removed during the night. Being elastic, it is apt to prevent the natural and proper circulation in the part.

Elastic knee-caps and anklets are helpful more as a placebo than as definite means of support; by their presence they seem to remind the athlete of their meaning on his return to sport. They may be employed during active and violent exercises when these are first resumed after an injury, but should be removed as soon as the exercises are over. They 


\section{SPECIAL IIETHODS OF TREATMENT}

must not be worn continuously or for any considerable time, and as a rule should be aroided whenever possible.

\section{(3) Mechanical Supports}

Leather straps to the wrist are not recommended.

Instruments for preventing displacement of the semilunar cartilages, and highly recommended by some, should be avoided as much as possible. They eventually lead to atrophy of the thigh muscles, and are only helpful to those who, for some definite reason, are unable to undergo an operation. The supposition that they retain the cartilage in position is absurd, since the structure is almost invariably displaced inwards into the inter-condyloid notch and almost never outwards.

Their action depends entirely on reducing the knee to a hinge, disallowing all rotation, and the general utility depends upon the accuracy of the fit of the circular bands around the thigh and leg. The hinge of the apparatus readily allows of flexion and extension.

The small 'spring trusses' for the knee, advertised and recommended in some quarters, are absolutely useless, since they are worn on the assumption that the semilunar cartilage is displaced from the knee. This is an error readily exposed both by pathological data and clinical experience.

It may here be stated as a general dictum, that every apparatus or appliance that can be dispensed with should be done away with on the earliest possible occasion when dealing with injured joints. 


\section{Of Plaster for Strapping}

several kinds are useful, none are ideal. The essentials of a good plaster are strength, a rubber base to prevent slipping, and perforations to allow of evaporation from the skin.

The plaster made by Messrs. Ewen and Sons, and spread upon chamois leather, is an excellent strapping, but it is rather expensive and somewhat irritating unless removed frequently.

Johnson and Johnson's ' Z O' (Zinc Oxide) plaster is very useful. It causes less irritation than most others, and is sufficiently firm.

'Leucoplast' or White Rubber adhesive plaster (Pilot Brand) is helpful. It answers most requirements and is not expensive.

Seabury's Rubber Adhesive Plaster, when perforated, is particularly useful for strapping the muscles of the thigh; but, even though it is perforated and allows of some evaporation, will produce irritation in most skins if left on for more than a few days.

In applying strapping, certain details must be attended to if success is to be attained. The limb or part of the body to be strapped must be cleanly shaved, then washed with soap and warm water, and subsequently cleansed with ether or benzine, and carefully dried. This is essential if the plaster is to remain in situ for several days.

The accompanying figures illustrate the writer's plan of strapping some of the commonest sprains in the body.

In strapping for a sprain of the chest (intercostal muscles), Fig. $75 \mathrm{~A}$, the strips of plaster should be 


\section{SPECIAL METHODS OF TREATMENT}

from $1 \frac{1}{2}$ to 2 inches wide, and made long enough to encircle the body to at least two-thirds of its circumference. Each horizontal strip, beginning from above and passing downwards, should cover half of the one immediately above it, and must be applied at the end of the act of expiration. The strapping should

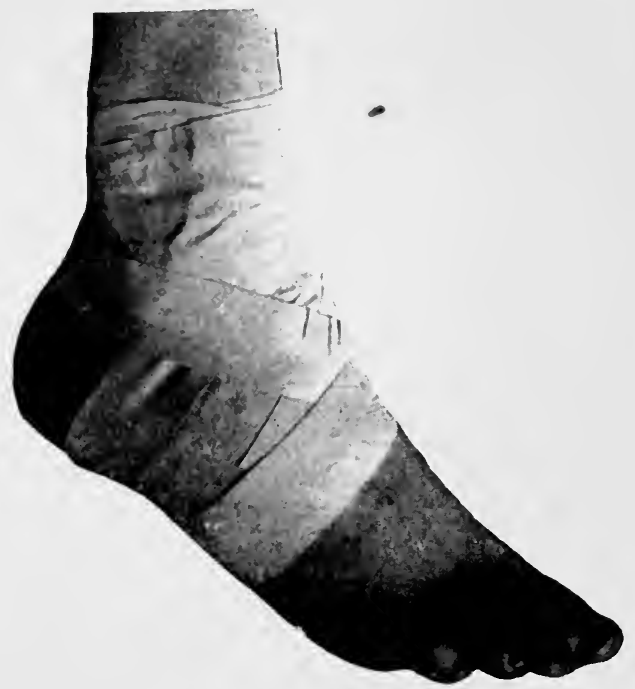

FiG. 83. Photograph of ankle strapped for strain by author's method. The plaster should begin nearer the ball of the toes.

extend for some inches both above and below the injury.

In 'Bowler's side', Fig. 75 в (strain of the oblique abdominal muscles), the same plan as the above should be adopted, and the strapping should extend from three inches above the level of the twelfth rib to well below the iliac crest.

The rectus abdominis (Fig. 74) is best strapped with a wide piece of Ewen's chamois leather plaster extending from the upper thigh, passing by Poupart's 


\section{SPECIAL METHODS OF TREATMENT 257}

ligament, to the front of the chest wall and slightly across the middle line of the body. Narrow overlapping strips, may, however, be employed with equal efficiency.

The muscles of the thigh (extensors, adductors, and hamstrings) are conveniently supported by a

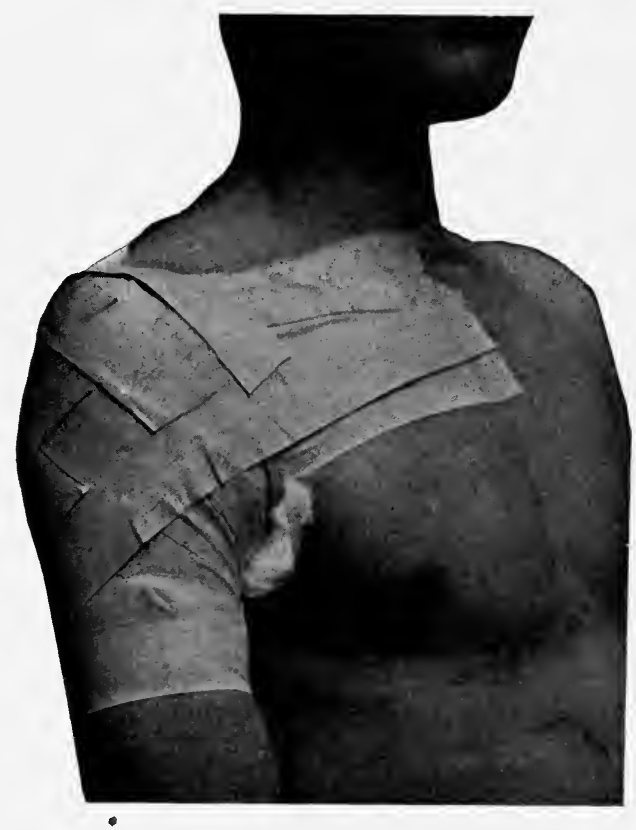

FIG. 84. To illustrate plan of strapping the shoulder for contusion or sprain. The axilla should be well padded, and the plaster extend to the middle line of body.

wide strip of Seabury's perforated plaster, not less than six inches in width, and made to encircle the limb for the greatest part of its circumference. It should not be made to meet, however (vide Fig. 78), or the muscles may be torn during vigorous contractions.

The muscles of the calf (soleus and plantaris) are THITELOCKE 


\section{SPECIAL METHODS OF TREATMENT}

strapped from below upwards, beginning at the smallest part of the leg and extending up to two inches below the knee. The strips of plaster will vary in width according to the size of the limb, and overlap from below upwards. The limb should

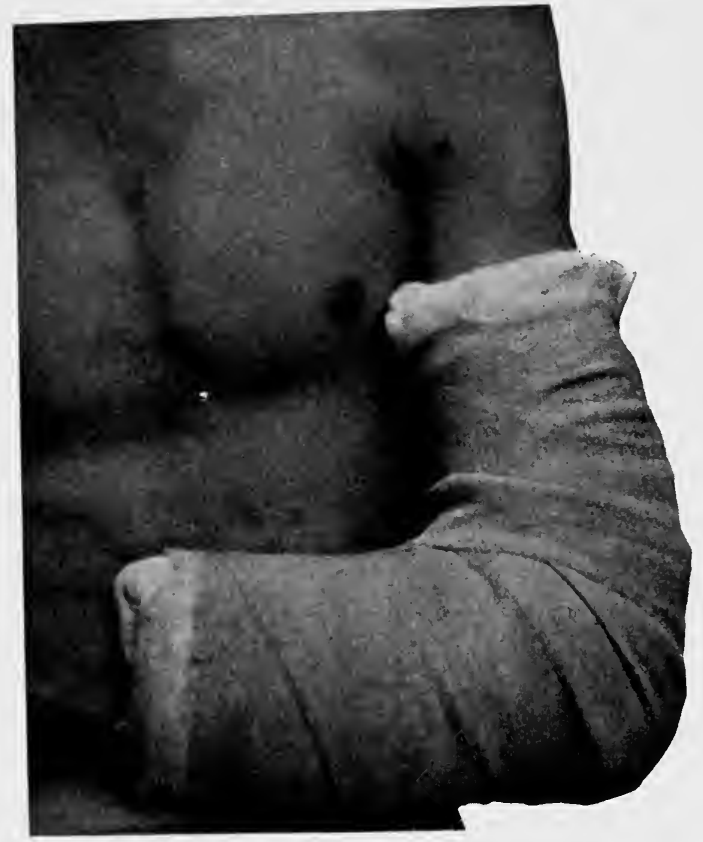

Fig. 85. An elbow-joint with elastic pressure applied for sprain with effusion.

always be depleted of as much blood as possible, by elevating it before the strips are laid on. Unless this precaution is taken, the plaster will soon become loosened by reason of the absorption of the blood which was effused.

Strapping of the knee, ankle, and feet is done on general principles. 
The deltoid and shoulder region should be accurately strapped by means of a spica, the ends of the plaster extending to the middle line of the body, both before and behind; while the axilla must be carefully padded with thick layers of wadding.

The other joints of the upper extremity, such as the elbow, wrist, carpo-metacarpal and interphalangeal, are dealt with on general lines.

The figures show also, in a graphic way, the method of applying elastic pressure to the knee- and elbowjoints ; the details of the process have been described in Chapter II.

The application of high degrees of temperature to sprains has, in the hands of some, met with much favour. Radiant-heat baths are extolled by many surgeons. They are said to diminish pain and to reduce the swellings, both intra- and extra-articular, in recent sprains. The writer's experience of their utility has been for the most part confined to joints stiffened as the result of early mismanagement. In a few cases improvement has resulted, but the process of cure has not been as quick as was expected. In some cases an extensive trial proved disappointing.

\section{Bier's Hyperemic Treatment}

Bier's hyperæmic treatment has undoubtedly been of service as a method in the treatment of sprains. Its chief value lies in the power it possesses in hastening the absorption of the extravasation both intraand extra-articular.

Daily applications of hot air for periods of an hour or less have been found both convenient and 


\section{SPECIAL METHODS OF TREATMENT}

efficacious by some clinicians where rapidity of absorption was an urgent desideratum. Patients often describe the applications as being soothing and comforting.

Active hyperæmia is superior to prolonged obstruc tive hyperæmia, even when the latter is either preceded or followed by massage. It is less uncomfortable and more easily applied. The necessary apparatus is simple. A wooden or metallic box or chamber may be so arranged as to partially or completely enclose the joint or limb. The hot air is generated by gas or electricity and passed as a current into the chamber in which there are arrangements to prevent scorching, and for equalizing the distribution of the temperature.

It is claimed that if such a condition as acute articular hydrops of the knee is treated immediately by this plan the effusion will be removed in the space of a few days. During the treatment the patient must be kept quiet in bed, or on a couch. As soon as the effusion has disappeared he may be allowed to exercise the limb to a certain extent, the joint being supported by a domette or flannel bandage, so as to allow of gentle movements of flexion and extension. While in many cases this plan has succeeded, there are others in which it has completely failed. Relapses are not uncommon after any form of hot-air treatment. The results obtained are not to be compared with those obtained by elastic pressure either as to rapidity, efficiency, or simplicity.

Obstructive hyperæmia has been useful in a few cases of relapsing traumatic articular synovitis in which there was difficulty in removing the chronic 
effusion. In all fairness it must be stated that the advocates of the hyperæmic treatment do not claim that it is a method which will supersede all others, but rather that it is a method which will be found helpful in a certain number of cases. Of this there can be no doubt, and the method seems to open up further possibilities.

\section{Mechano-Therapy}

In recent years in America, as well as on the Continent of Europe, the treatment of sprains by mechanical means has received definite and authoritative support. The term mechano-therapy, properly speaking, covers a large field. Whilst some clinicians include massage in its various forms and passive movements under this definition, there are others who use the expression in a stricter sense, and confine its use only to cases treated with the aid of mechanical apparatus. In its application to the treatment of sprains the value lies more in the direction of overcoming stiffness and atrophy in the latest stages of the injury than in the earlier management. Few will deny that side-to-side or to-and-fro pendulum-like movements, even if executed with the aid of machinery, are useful in stretching or even breaking down adhesions. That they encourage nervous patients to exercise their limbs more freely than they would otherwise must be conceded, but when employed as a means of overcoming atrophy in muscles, or in promoting their rapid regeneration, it is doubtful if any mechanical means will be as efficacious as voluntary active movements. 


\section{SPECIAL METHODS OF TREATMENT}

For the regeneration of atrophied and shrunken muscles something more than mere mechanical exercise is needed, and that something may be best described as nervous impulse. The natural passage of a nervous impulse from the brain to the injured muscles or other tissues, so influences their general nutrition as to render them more readily responsive to their physiological requirements. This should be the primary aim of all exercises. Active muscular exercises, whether performed by weight-lifting or by pulling against resistance, are more valuable for this selfsame reason. Patients should be taught to realize that the natural everyday movements, however gently performed, will be in themselves sufficient to prevent either stiffness or shrinking.

Mechano-therapy will be of service chiefly in those cases that have been previously mismanaged. Vibration instruments worked by electricity were introduced under the name of seismo-therapy. They afford a convenient means of applying deep friction to certain parts of the body, in which other forms of friction-massage are unsuited or impracticable.

\section{Injections of Fibrolysin}

The injection of fibrolysin for the removal of adhesions, and as a means of rendering stiff joints more supple and movable, has been tried. The method is still on its trial. The earlier expectations have hardly been realized. Injections of fibrolysin of themselves are not sufficient as a therapeutic system; their injection alone will not result in benefit. They must be supplemented by massage and movements, 


\section{SPECIAL METHODS OF TREATMENT 263}

and would seem to be useful mainly by softening the fibrous tissues. The softening of adhesions and other fibrous bands allow of their being more readily dealt with afterwards by manipulation. The injections have been tried in almost every form of fibrosis, and with varying results. It is best injected deeply into the muscles of the gluteal or deltoid regions.

Dr. R. H. Strong ${ }^{1}$ recorded as his experience of the drug, that after its use there was ' not only in all cases a local improvement but there was considerable improvement in the general health of the patient'.

$\mathrm{He}$ noted also some disadvantages. After an injection there is a reaction, and the temperature rises to about $100^{\circ} \mathrm{F}$.; and in about fifteen minutes after an injection an odour of garlic could often be detected in the breath, whilst a peculiar bitter taste, which lasted for some hours, was frequently complained of. The writer has heard of a case of fatal purpura, which was supposed to have followed the injections of the drug. Treatment of sprains by fibrolysin injection in our present state of knowledge cannot be much recommended.

\section{KATAPHORESIS}

One of the most important and constant actions of the electrolytic treatment is its resolving influence on cicatricial formations when a kathodal stream of a solution of sodium chloride is employed. Joints completely ankylosed have been known to recover

1 Lancet, August 21, 1909, p. 529. 


\section{SPECIAL METHODS OF TREATMENT}

rapidly their mobility without forced movements or pain.

Professor Leone ${ }^{1}$ writes, 'The resolving action of electrolysis suitably applied is not a matter of chance. It is regular and sure. No other means employed in medicine can be compared with it for efficacy, rapidity, perfection of results, and absence of pain.' No one is, probably, better able to speak authoritatively concerning the merits of the system than this French professor, who for many years has employed it as a therapeutic measure.

Any power that is capable of influencing the tissues as the advocates claim for this one, cannot fail to be of service in the management of stiff joints. It is to this particular result or sequela of sprains that the method is best adapted. Even firmly ankylosed joints are said to be amenable. As the practice of kataphoresis requires special apparatus it necessarily follows that its general use must be restricted. It can hardly be within the immediate reach of every clinician. In this country there are already institutions properly equipped for carrying out all the details of the electrolytic treatment. Old cases of sprain complicated with gout, rheumatism, or other constitutional affections may be readily and conveniently treated by the administration of their specific remedies by the electrolytic method.

Superficially placed injuries are more speedily and with more certainty acted upon than are those deeply placed. An exposed joint, such as the elbow or ankle, is more amenable than the shoulder or hip.

${ }^{1}$ Electric Ions and their use in Medicine. English translation. Rebman Limited, 1908, p. 44. 


\section{SPECIAL METHODS OF TREATMENT 265}

The muscles which envelop or cover over a deeply situated injury are such good conductors that they serve to deflect the current and diminish its penetrating force.

Kataphoresis as a therapeutic agency is destined to play an important part in the medicine of the future, and its application to the management of traumatic conditions of the joints will probably prove of great service. 



\section{INDEX}

Acromio-clavicular joint, sprains of, 79.

causes of, 79 .

guarded prognosis in, 79 .

symptoms, diagnosis, and treatment of, 79 .

Active voluntary movements, in treatment of sprains, 26, 85, 244.

early uses and advantages of, 26 , 244-6.

limitations of, 244, 246.

method of employment, 244.

physiological action of, 244.

Acute traumatic synovitis of knee, prognosis of, 127.

treatment of, 127.

Adductor longus muscle, 43, 66 .

rupture of, 230 .

causes, symptoms, diagnosis, and treatment of, 201-2.

Adductor magnus tendon, strain of, 231.

Alar ligaments, 48, 74, 75, 180, 193.

Albuminuria, as a complication of sprains, 19.

Anæsthetics, in treatment of sprains, 14.

contra-indications of, 14 .

for diagnosis, 32 .

use of, in aspiration, 32.

use of, in synovitis, 46 .

Anatomy, general, of joints, 2 . surgical, of knee-joint, 120, 124.

'Angler's elbow,' 194.

Ankle-joint, sprains of, advantages of elastic pressure in, 114.

after-treatment of, 114 .

causes of, 112.

complications in, 112, 115.

differential diagnosis of, 112.

frequency of, 112.

method of strapping in, 114, 253.
Ankle-joint, sprains of, prognos is and treatment of, 112, 114. sprain-fracture of, 112. symptoms of, 112.

surgical anatomy of, 112.

Anklets, elastic, 253.

Ankylosis, as sequela of sprainfracture, 41.

causes of, 60 .

treatment of, 60 .

Annandale, Professor, the late, 188.

Anterior annular ligament, 112.

Anterior crucial ligament of the knee, 122, 154.

Apparatus for preventing displacement of semilunar cartilage, 254.

Arthritis deformans, 71 .

Arthrotomy, and temporary drainage of joints, 34, 50, 182.

author's operation for, 177 .

for digital exploration in recurrent synovitis, 34 .

for removal of cartilages and loose bodies, 175-6.

in treatment of joints, 34 .

temperature charts in, 49, 184-7.

Articular sprains, 8.

classification of, 9 .

individual articular sprains, 78 .

Articular vessels, 3.

Aspiration, in treatment of synovitis and tension in joints, 32 .

for large blood extravasations, 83.

indications for, 32 .

special advantages of, 33 .

Aspirin, in treatment of nerve pain, 53.

Athletics, as a factor of sprains, 6 .

Atrophy of muscles, acting on joints $1,43$.

as a sequela of articular sprains, 41. 
Atrophy of muscles, as the result of nerve injury, 43.

from prolonged fixation in splints, 43.

Atrophy from disuse, 44.

means adopted for preventing, 85 . treatment of, by active voluntary movements and exercises, 245 , 246 .

Bandages, advantages of intermittent removal and reapplication of, 24 .

' crêpe velpeau,' $23,28$.

special, domette, elastic webbing, plain rubber, $23,253$.

spica, use of, in sprain of shoulderjoint. 8.2 .

Bennett, Professor, of Dublin, 105.

Bennett, Sir William. 134.

Biceps brachialis, rupture of, 218 .

Biceps cruris muscle, rupture of, 229.

Biceps flexor cubiti muscle, rupture of, 220 .

diagnosis and treatment of, 221 .

Bicipital groove, 220.

Bier's hyperamic treatment, 45, 259. active hyperæmia, 260.

methods of application of, 260 .

obstructive hyperæmia, 260.

special advantages of, 260 .

uses of, 259-60.

Blistering in treatment of nerve lesions, 37.

Bone-setters, treatment of sprains by indiscriminate movements and rubbings, 1, 6, 7, 36, 207.

Bones entering into formation of joints, 2.

'Bowler's side,' 195, 211, 217.

method of strapping for, 256 .

Brachial plexus, injuries of, 13.

Breaking-down, in treatment of ankylosis, 60 .

in treatment of stiff shoulderjoint, 84.

Brunton, Sir Lauder, Bart., 241.

'Calcareous deposition,' in detached semilunar cartilage, 76 .

in muscles, 67.

possible forerunner of other osteoarthritic changes, 76.
Calico bandage, 23.

Callus, in sprain-fracture, increased by movement and friction, 1 .

Callus-formation at seat of fractures, 64 .

Capsular ligament, endothelium of, 2. linings of, 2.

of knee-joint, 120.

of shoulder, 80.

of sterno-clavicular, 78 .

structure of, 2.

Carpal bones, fracture of, 93 .

cases illustrative of, 98-100.

Carpal joints, 4.

Carpo-metacarpal joints, injury of, in sprains of thumb, 104, 105.

Cartilage: semilunar cartilages of knee, anatomy of, $153,154,156$, 157.

displacement of internal semilunar, 74, 148, 149, 152.

author's method for reducing displacement, 162-3.

author's operation for, 177-82.

differential diagnosis of, 161 .

operative treatment of, 174 .

preliminaries to operation, 177.

symptoms and clinical characters of, 157-8.

treatment of recurring or relapsing displacement of, 173.

hyaline, growth of, in detached portions within the capsule of a joint, 73, 171.

inter-articular, 5 .

loose in joints (see Loose Bodies), 75 .

Cases illustrating treatment of-

displaced internal semilunar cartilage illustrated by temperature charts, 184-7.

displaced peroneal tendons, 199203.

displacement of internal semilunar cartilage in footballplayer, 150.

displacement of small tendons at back of neck, 204-6.

fracture of metacarpal bone of thumb, 106.

fracture of scaphoid bone, 98100.

longitudinal splitting of an internal semilunar cartilage, 152-3. 
Cases illustrating treatment 0 loose bodies in joints, 74 . ossifying myositis, $64,65,66$. rupture of tendo-Achillis, 236. separation of epiphysis of anterior inferior spine of ilium, 222. sprains, rheumatism as a complication of, 29.

'wobbly joint,' 50.

Charcot's disease, 71, 247.

Charts, temperature, in arthrotomy, 184-7.

Chronic and relapsing synovitis, treatment of, 127.

Chronic atrophic neuritis, 12.

Clavicular joints, 4.

inter-articular cartilage of, 12.

Coccygodynia, a symptom in sacrococcygeal sprain, 119.

electrical vibration for relief of, 119.

Coccyx, fracture or dislocation of, 118.

Cold, in treatment of sprains, 19. contra-indications of, in debilitating diseases, 19.

Colles's fracture, 92.

Complications of sprains, 9, 10.

with gout, 30 .

with injury to nerves, 12, 35 .

with rheumatism, 29.

with tuberculosis, 30 .

with tumour, 16.

Constitutional complications of sprains, 30.

treatment of rheumatic, gouty, syphilitic, and hæmophilia, 30.

Contusions, elastic pressure in treatment of, 20.

Corpora oryzoidea, 71.

Crêpe velpeau bandages, 23, 28.

Crepitus, occasional absence in fracture, 15.

present in osteo-arthritis occasionally, 15.

value as diagnostic sign in sprain, 15.

' Cricket shoulder,' 211. nature of injury, 219-20. treatment of, 220.

' Cricket thigh,' 222.

Crippled or deformed joint as sequela of sprain, 41 .

etiology of, 54 .
Crucial ligaments, anatomy of, 122. Crureus muscle, 64 .

Defæcation, pain during, 118.

Deferred swelling in joints, 10.

Deformed joint as sequela of sprain, etiology of, 55 .

Deltoid muscle, 60.

causes of wasting in, 43 .

oversprain of, or partial rupture of, 43.

rupture of, 218.

Deltoid, sub-, bursa, enlargement of, 81.

Diagnosis, modern methods of, in sprains, 14.

Dislocation, 4.

' by elongation,' symptoms of, 88 .

injury of wrist in, 89.

diagnosis of, 89.

prognosis of, 89.

treatment of, 89.

of semilunar cartilages, 147.

anatomical reasons for greater

frequency of internal displacement, 158.

treatment of, 162-70.

of tendons, 198.

with sprain, 12.

Displaced external semilunar cartilage, 188.

cases illustrative of, 188-92.

diagnosis of, 191.

pathology of, 188-9.

treatment of, 190-1.

Displaced internal semilunar cartilage, author's special method for reduction of, 163-6.

causes of, 161 .

differential diagnosis of, 161 .

early treatment of, 162-6.

popular devices for reduction of, $162-6$.

subsequent treatment of, 166-73. symptoms and clinical characteristics of, 157.

temperature charts in operation for removal of, 184-7.

Displacement of tendons, 198.

biceps flexor cubiti, 199, 203.

peroneal tendons, 202-3.

peroneus brevis, peroneus longus, 198, 200.

short tendons of neck, 204-6. 
Displacement of tibialis posticus, 198, 202.

Distal muscles of a joint, hypertrophy of, 52 .

Domette bandage, 23.

Drainage, in arthrotomy, 48, 182. for chronic synovitis, 48 . for exploration of knee, 48 .

Drainage for removal of displaced semilunar cartilage, 181.

'Driver's elbow,' 194.

'Drop-top,' anatomy of, 106. author's operation for, 108-9. deformity in consequence of, 106. how caused, 106. symptoms of, 106. treatment of, 107-8.

Dumbbells, uses of, for developing muscles, 85.

Ehrlich, Professor, 208.

Elastic anklets, 253.

Elastic knee-caps, 253.

Elastic pressure in treatment of sprains, 20, 255-9.

advantage of, in sprains of ankle, 114.

compared with massage, 245 .

contusions, 20.

in tension of joints, 33 .

in treatment of fracture-sprains, 38.

in treatment of synovitis, 46 . principles for applying, 20, 22 . special advantages of, 20 .

universal application of, in cases of extravasation, 20,24 .

Elastic rubber bandage, contraindications of, in sprains, 25.

Elastic webbing, 253.

Elbow-joint, hinge of, 4.

orbicular ligament of, 86 .

sprain of, 85 .

differential diagnosis, treatment, and prognosis of, 87. surgical pathology of, 86 .

treatment of sprain of, by elastic compression, 259.

Electric ions, use in medicine, 264.

Electrical stimulation, in treatment of localised palsy and paresis, 62.

in treatment of general injuries to muscles, 195, 198.
Electrical stimulation, in treatment of sacro-coccygeal sprain, 119.

in treatment of sprains of the ankle-joint, 116.

Electrolysis, resolving action of, in treatment of sprains, 264.

Electrolytic treatment, 263.

Ely, Dr. L. W., 119.

Endothelium, secreting function of, 2.

Epiphysis for anterior inferior spine of ilium, separation of, 222.

cases illustrating, 222-3.

Evaporating lotions in treatment of sprains and contusions, 51 .

Exercises, against resistance, 85,250. by means of weights and pulleys, 85.

indications for, in fracture-sprain, $38,40$.

Exostoses, as cause of knee sprain, 142.

diagnosis and treatment of, 144.

' snapping sound' caused by the presence of, 144.

symptoms of sprain with, 143.

synovial bursæ in relation to, 143 .

Exploratory arthrotomy, 34. temperature charts for, 49.

External lateral ligament of knee, sprains of, 132.

prognosis and treatment of, 133. symptoms of, 133.

External semilunar cartilage of knee, anatomy of, 153-7. displacement of, 188.

Extravasation, aspiration for, 83. elastic pressure as a means of checking, 20, 24.

in articular sprains, 24.

in muscular sprains, 24.

Ewen's plaster, 255.

Faradic current, in treatment of adhesions, 198.

Fatigue, to be avoided while practising movements and exercises, 85.

Femur, internal and external condyles of, 122 .

Fibrinous loose bodies, 71 .

Fibrolysin, injections of, 262.

disadvantages of, 263 .

uses and limitations of, 262, 263. 
Fixed apparatus, disadvantages of, in treatment of sprains, 26,28 .

'Floating patella,' as symptom in sprain of knee, 124.

value of, 125 .

Fomentations with anodyne lotions for relief of tension, 33 .

'Footballer's knee,' 127.

Forcible movements of stiff joints under an anæsthetic, 246. contra-indications for, 247.

indications for, 247.

limitations of, 246.

rules governing use of, 247-8. special advantages of, 246 .

Fracture of first phalanx of little finger, 109.

deformity as result, 109.

difficulty in diagnosis of, 109. treatment of, 109.

Fracture-sprain, 10. causes of imperfect recovery, 38. of metacarpal bone of thumb, 104. of wrist-joint, 91-6. treatment of, 38 .

treatment of, compared with that of simple sprain, 39.

$\mathrm{X}$-rays in diagnosis of, 8.

Friction massage, 36, 138, 241.

Gamgee-tissue, 28.

as application in elastic compression, 23.

Gastrocnemius muscle, in case of ossifying myositis, 66.

General injuries to muscles and tendons, 194-214.

Genu-valgum or genu-varum, as result of sprained knee, 56 . etiology of, 56 .

Gluteal muscles, 55, 110.

Glycosuria as a complication of sprains, 19.

Godlee, Professor R. J., 188.

Gout as a complication of sprains, 30.

Gymnasium, exercises at, 85, 246, 250.

Hæmatomata in sprains, 10 .

as complication, 14.

operation for large, 83.

Hæmophilia as complication of sprains, 29,30 .
Hæmorrhage, in articular and muscular sprains, 9.

means of checking, 20.

Haglund, Dr., 208.

Hamstring muscles, rupture of, 229. usual sites of, 229.

Hamstring muscles, causes, symptoms, prognosis, and treatment of, 229-30.

Heat in treatment of sprains, 33 .

Hernia of muscles, 91, 195.

adductor longus, 196.

rectus abdominis, 196.

rectus femoris, 196 .

tensor vaginæ femoris, 196.

tibialis anticus, 196.

High degrees of temperature, application of, 259.

uses of, 259.

Hip, contusions of, treatment of, 111.

Hip, sprains of, 109.

causes of, 109.

difficulty of diagnosis in, 110. osteo-arthritic changes in, 111.

painful nature of, 109 .

prognosis and treatment of, 110, 111.

symptoms of, 109.

wasting of muscles in, 111.

Hip-joint, ball-and-socket arrangement of, 4.

Hot fomentations in treatment of sprains, 33 .

Hunter, John, 237, 239.

Hyaline cartilage, 102.

purposes of, in structure of joints, 2.

Hydrops, traumatic, 127.

Hypertrophy of synovial fringes, diagnosis of, 193.

operative treatment of, 193.

symptoms of, 192.

Iliacus muscle, 14.

Ilio-psoas muscle and tendon, rupture of, 218.

causes, symptoms, and treatment of, 218.

Immediate massage, 243.

Improper union of muscles and tendons, as sequela of sprains, $41,56$.

Indications for massage, 243. 
Individual injuries to muscles and tendons, 213-39.

Inflammation in joints, etiology of, 3:2.

Infra-patella pad, 45.

Injuries, diagnosis and treatment of, 1.

Inter-articular fibro-cartilage of knee, 15, 12.2, 155.

anatomical variations in, 124 .

special attachments to, 123 .

surgical anatomy of, 122.2.

Int?r-articular fibro-cartilage of sterno-clavicular joint, 78 .

Inter-muscular septa, overstretching and tearing of, in elbowsprain, 91.

Internal derangement of knee-joint, 147.

causation of, 147 .

common site for laceration of internal cartilage, 148.

surgical pathology of, 147 .

terms synonymous with, 147 .

Internal condyle of femur, 122.

Internal lateral ligament of knee, $1 \cdot 21$.

causes of, 130 .

diagnosis and treatment of, 132.

rupture of, 130.

symptoms of, 130.

usual sites of laceration in, 130 .

Internal semilunar cartilage, anatomy of, 153 .

longitudinal splitting of, 151 .

more liable to injury than external, factorsconcerned in, 155 .

lodine, liniment and tincture of, 2.2 .2$.

Johnson and Johnson's plaster, 225, $25 \overline{5}$.

Joint, cavity of, 3.

Joint, distension of, from injury or disease, 3.

persistent pain in, 53.

Joints, adhesions within, 2.

blood-vessels of, 3.

capsule of, 2 .

causes of neuralgia in, 53 .

crippled and detormed as sequela to sprain, 54 .

gliding movements as a source of strength of, 5 .
Joints, inter-articular cartilages of, 5.

loose bodies in, 16, 70, 71, 76 .

nervous supply of, physiological relation of, 3 .

stability and strength dependent upon, 3.

structure and function of, 2.

tension in, 31 .

total disablement of, 2 .

'weak joint,' 50.

'wobbly joint,' 50.

'Jumper's (broad) sprain,' 194, 229.

Kataphoresis, 45, 263.

actions and uses of, 263, 264.

as a therapeutic agency, 265 .

Knee-joint, 4.

after-effects of sprain of, 127 .

capsular ligament of, 120.

coronary ligaments of, 124.

crucial ligaments of, 122.

differential diagnosis of sprain of, 125-6.

disorganization of, as a result of entorced exercise, 16, 17.

internal derangements of, 147. internal lateral ligament of, 121. long external lateral ligament of, $1 \cdot 21$.

open operation not to be lightly considered, 34 .

patellar ligament of, 121.

pedunculated bodies in, 193.

posterior ligament of, 121 .

quadriceps extensor tendon of inter-articular fibro-cartilage of, 122.

sprains in region of, 120 .

sprains of, 111, 120.

surgical anatomy of, 120.

treatment and prognosis of sprain of, 127.

treatment of sprain of, by elastic pressure, 22, 23.

usual site of pain in, 126.

König, Professor, 73.

'Labourer's back,' 195.

Lange, Dr. S., 207.

Langton, Mr, J., 188.

Laparotomy, 181.

'Lawn-tennis leg,' 211. nature of injury, 233 . 
'Lawn-tennis leg,' symptoms, diagnosis, prognosis, and treatment of, 234-6.

Leather straps for wrist, 254.

Leone, Professor, 264.

Leslie's plaster, 18.

Leucoplast, 18, 255.

Ligamenta alaria, 48, 74, 75, 180 , 193.

conversion of into fibrous bands, 75 .

photograph of specimen removed by operation, 74 .

Ligamentum mucosum, 48, 180, 193.

Ligaments, capsular, of knee-joint, 120.

coronary, of knee, 124.

crucial, of knee, 122, 123, 154.

external lateral, of knee, 132.

injury of, in elbow-joint, 86.

inter-articular, 2.

internal lateral, of knee, 121, 126, 130.

long external lateral, of knee, 121, 132.

of sacro-iliac joints, 118.

ossification of, 3 .

patellar, of knee, 121, 228, 231, 232.

posterior, of knee, 121.

stretching and elongation of, from distension of joint cavity, 3 .

Lindsey \& Sons, Messrs., 174.

Liniments in use, 252.

Local applications in treatment of sprains and injuries to joints, 252.

Local depletion and flushing in treatment, 36.

Local heat a symptom of tension in joints, 32 .

Localised palsy and paresis as sequelæ of sprains, 61 .

Long external lateral ligament of knee, 121.

Longitudinal splitting of internal semilunar cartilage, 151.

Loose bodies in joints, 16, 61, 70, $71,76,77$.

classification of, 70, 71 .

commoner varieties of, 76 .

developing in ligamenta alaria, 75.

WHITELOCKE
Loose bodies in joints, etiology of, $70,71,72,73,76$.

microscopical appearance of, 76 . pedunculated loose bodies, 75 .

preliminaries to operative treatment of, 77.

symptoms and treatment of, 77 .

Lower extremity, rupture of muscles of, 222.

Malleoli, 112, 114, 201.

'Mallet-finger,' anatomy of, 106.

author's operation for, 108-9.

deformity in consequence of, 106 .

how caused, 106.

photographs of, 107.

symptoms of, 106.

treatment of, 107-8.

Marsh, Professor Howard, 200.

Massage, for reducing pain, 18.

indications and contra-indications of, 242-3.

in treatment of fracture-sprain, $38,40$.

in treatment of sprains, 240 .

mechanical action of, 241.

physiology of, 240.

supposed disadvantages of, 25 .

uses of, as an assistant to elastic pressure, 25.

when and how to apply, 26.

Massage movements and exercises in treatment of sprains and bruises, 240.

Mead's plaster, 18.

Mechano-therapy, 246-61. uses and limitations of, 261-2.

Medical practitioner, treatment of sprains by, 1 .

Mercury, uses of, in constitutional complications, 30 .

Mesoblast, common ancestor of, 3 . structures in joints derived from, 30.

Metacarpal bone, Bennett's fracture of, 105.

photographs of, 103-5.

linear fracture of metacarpal bone of thumb, 105.

Metacarpo-phalangeal joints, injury of, in sprains of thumb, 104, 105.

Metatarsal pain, causes of, 115. description of, 115. 
Metatarsal pain. symptoms and treatment of, 1i5-16.

Metatarso-phalangeal joints. 115 .

Minor surgery, 'little things of,' 6 . importance of, 6 .

Ilorements and exercises, in treatment of sprains and bruises, $240-5)$.

against resistance, 250,251 .

Mucin, 2.

Murphy. Mr. J. Keogh, 57, 58-87.

Muscle hernia, 91, 195.

Muscle sprain, 9. 194.

examples of, 194-5.

symptoms and treatment of, 195.

Muscle strain, 195.

Muscle-wasting, as a result of lesions of nerves, 37 .

as a sequela of sprains, 41 .

as a serious complication of sprains, 26.

as result of treatment by splints, 26.

author's means of preventing, 248-51.

caused by injury to nerve supply, 43.

and from disuse, 43.

causes of, 43.

following immediately upon injury, 43.

Muscles, atrophy of, acting on joints, 2. 43.

hernia of, 91.

causes of, 195 .

examples of, 196.

symptoms, diagnosis, and treatment of, $197-8$.

of calf, mode of strapping, 257-8.

of chest, mode of strapping, 255-6.

of lower extremity, rupture of, 2:2:2.

of thigh, mode of strapping, 257.

of trunk, ruptures of, 215 .

operation for suturing, 225.

ossification of, 67 .

regeneration of atrophied, 85 .

Muscles, injured, and in region of injuries-

adductor longus, 43, 66, 67, 230 .

adductor magnus, 231.

crureus, 64 .

deltoid, $43,56,81,83$.
Muscles, injured, and in region of injuries-

extensor carpi radiales longiores et breviores, 90 .

gastrocnemius, 66 .

gluteal, 54, 110.

hamstring, 229 .

iliacus, 31 .

intra-spinatus, 81 .

oblique, of abdomen, 216.

pectineus, 66.

plantaris, $211,233$.

popliteus, 133.

pronator radii teres, 194 .

proximal, 52.

psoas, 31 .

quadriceps extensor, 64, 228.

rectus abdominis, $31,196,216,256$.

rectus femoris, $60,222,224$.

sacro-lumbalis, 35, 195, 206, 218.

soleus, 65,66 .

sub-deltoid bursa, 81,83 .

supinator longus, 90.

supra-spinatus, 81 .

tensor vaginæ femoris, 196.

the peronei, 199.

tibialis anticus, 196.

triceps, 218.

Muscles and tendons, general injuries to, 194-214.

individual injuries to, 215-39.

Myositis ossificans as sequela, 62, 64 . causes of, in Schlatter's disease, 139.

in patellar ligament, in Schlatter's disease, 138-40.

treatment of, 66-8.

Nation, wage-earning capacity of, 6 .

Nerve, circumflex, pressure upon, cause of pain in sprain of shoulder-joint, 81.

sciatic, injury to, 13.

Nerves, lesions of, as complication of sprains, 12, 35 .

absolute and complete rest often imperative, 36 .

blistering in treatment of, 37 .

difficulty of early diagnosis of, 35 . importance of seeking for, 35 . local neuritis as result of, 37 . massage in treatment of, 36 . numbness and tingling as symptoms of, 35 . 
Nerves, lesions of, opiates contraindicated in, 37.

pain in, as result of pressure, 53 . static electricity in treatment of, 37.

symptoms due to tearing of, 35 . treatment of, 36 .

wasting of muscles in, 37 .

Neuralgia, causes of, in painful joints, 53.

treatment of, 53 .

Neuritis, as a cause of persistent pain in a joint, 53.

as complication of sprain of shoulder-joint, 81.

chronic atrophic, 12.

local treatment of, 37 .

Nutrition of joints, influenced by alternate flushing and depletion in treatment by elastic pressure, 24.

Oblique muscles of abdomen, rupture of, 216.

causes, symptoms, prognosis, and treatment of, $216,217$.

Open operation, not to be lightly considered, 34 .

often only means of cure, 34 .

Operation, for displaced semilunar cartilage, author's method of, 177.

for displacement of peroneal tendons, 203.

for 'drop top ' or ' mallet-finger', 107-8.

for exploratory arthrotomy, 34 .

for large hæmatomata, 83.

for rupture of quadriceps extensor, 228.

for ruptured patellar ligament, 232.

for suturing muscles, 225.

Orbicular ligament, of elbow-joint, 86.

Ossifying myositis, 61 .

diagnosis of, important, 67.

etiology of, 62.

in adductor longus muscle, 66 .

in crureus muscle, 64 .

in deltoid muscle, 66 .

in gastrocnemius muscle, 66 .

in pectineus muscle, 66 .

in quadratus extensor muscle, 64 .
Ossifying myositis, in soleus muscle, 65,66 .

osteo-blasts in production of, 64 . preventive and curative treatment of, 67-8.

structure of bone in, 64 .

symptoms of, 67.

usual situations of, 62 .

Ossification of muscle, symptoms of, 66, 67 .

mistaken for sarcoma of thigh, 67 .

Osteo-arthritis, as result of injury, 68.

as sequela, 16, 61, 68 .

calcareous deposition a possible forerunner of, 76 .

crepitus occasionally present in, 15.

etiology and pathology of, 69 .

treatment of, 69 .

Osteo-blasts, in production of ossifying myositis, 64 .

Osteo-chondritis dissecans, 73.

Paget, Sir James, 142.

Pain, as a symptom of tension in joints, 32 .

metatarsal, 115.

persistent, in joints, 41, 53 .

Painful and stiff joints, general causes of, 41-2.

Palsy, localised, as sequela of sprain, 61.

electrical treatment of, 62 .

etiology of, 61.

Papillæ, hypertrophied, of synovial membrane, in chronic synovitis, 75. See Villi.

Paralysis, local, as sequela of sprain 61.

Paresis, local, as sequela of sprain, 61.

Partial dislocation of the patella outwards, 144.

diagnosis of, 145 .

predisposing cause of, 144-6.

symptoms of, 145.

treatment of, 145-6.

Passive movements, comparison with active, in treatment of sprains, 27.

contra-indications of, 244.

in simple sprain, $27,244$.

in sprain-fracture, 38, 244. 
Pasive movements, limitations of, $2 \mathrm{~s}$.

mechanical action of, 28,244 .

special uses, 244 .

Patella, floating, 124-5.

Patellar ligament, rupture of, 2.28, 231.

operative treatment of, 232 .

symptoms, diagnosis, and treatment of, 231,2322 .

Pectineus muscle, 66. rupture of, 230 .

Pectoral muscle, rupture of, 218 .

Pectoralis major muscle, 2:20.

Pedunculated bodies in knee-joint, 193.

Periosteum, injury to, in ossifying myositis. $62.64,65,66$.

Peroneal tendons, displacement of, symptoms and treatment of, 203.

Persistent pain in joints, 41, 53.

Physiology of massage, 240 .

Plantaris muscle, rupture of, 211.

Plasters, Johnson and Johnson's, $2.25,25.5$.

Leslie's, 18.

Ilead's, 18.

Seabury's, 18, 2227, 255.

various, 2.55.

Points de feu, as method of treatment, 45 .

Poirier, Professor, 156.

Popliteus muscle, 133.

Popliteus tendon, 155.

Poppy-head lotion, 8:2.

Posterior erucial ligament of knee, at tachments of, 154.

Posterior ligament of knee, 121.

Poupart's ligament, 218, 230.

Preventible sequele of sprains, 41. enumeration of, 41.

Prevention of muscle-wasting by gentle, active, muscular exercises and training, 248 .

Prognosis in sprains, importance of, 16.

Pronator radii teres muscle, 194.

Proximal museles of a joint, atrophy in 5.2

Psoas muscle, 14, 35.

'Pulled elbow,' diagnosis, treatment, and prognosis of, 89 . injury of wrist in, 89 .
'Pulled elbow,' symptoms of, 88.

Pulleys, use of, 220 .

Quadriceps extensor muscle of thigh, $64,211$.

operation for rupture of, 228 .

Quadriceps extensor tendon, rupture of, 228 .

diagnosis and treatment of, 228 . operation for treatment of, 228-9.

Radcliffe Infirmary, 189.

Radiant heat in treatment of sprains, 33.

application of, 45.

Radiant heat baths, 259 .

Radiocarpal epiphysis, 93.

Radiography, 97, 98, 101.

Radioseopy, 87.

Range of movement in stiff joints, 247 .

Rawling, Mr. L. B., 158.

Rectus abdominis muscle, 31, 196. method of strapping, 217, 256 .

rupture of, 216. eauses, symptoms, and treatment of, 216.

Rectus femoris muscle, 60 . plaster strapping for, 2:25-6. rupture of, 224 .

symptoms and treatment of, $224-5$.

Recurrent or relapsing displacement of semilunar cartilage, 160 .

after-treatment of, 182-3.

author's method for removing, 177.

illustrations of, 178-80.

operative treatment of, indications for, 174.

palliative treatment of, 173 .

preliminaries to operation, 177.

Recurring and relapsing synovitis, $45-6,74$.

appearance of synovial membrane in, 48.

naked-eye and microscopic, 48. arthrotomy for digital exploration in, 34.

method of operation for, 48 .

nature of synovial secretion in cases of, 48.

relaxed state of ligaments in cases of, 52 . 
Recurring and relapsing synovitis, temperature charts in, 184-7.

Relapsing sprains, often dependent upon health of individual, 28.

Rest, in treatment of sprains, 41-2.

Restoration of mobility in injured joints, primary object in treatment, 26.

Rheumatism as complication of sprains, 29.

treatment of, 30 .

' Rice bodies' in joints as sequela of sprain, $71,76$.

'Rider's sprain,' 43, 194, 211, 230.

Rigid splints, uses of, contra-indicated in treatment of sprains, 26.

Rule-of-thumb methods, disadvantages of, $1,6,7$.

Rupture of muscles and tendons, athletic exercises in relation to, 212.

causes of, 209-14.

circumstances which influence tendency to, 212-14.

clinical symptoms of, 211 .

influenced by age of individual, 214.

by kind of game, 214 .

by season of year, 213.

by temperature of air, 213 .

by want of training, 213.

of adductor longus, 230 .

of adductor magnus, 231.

of hamstrings, 229.

of ilio-psoas, 218.

of lower extremity, 222.

of oblique muscles of abdomen, 216.

of patellar ligament, 231.

of plantaris, 233 .

of quadriceps extensor, 228.

of rectus abdominis, 216, 256.

of rectus femoris, 222 .

of sacro-lumbalis, 218 .

of soleus, 233.

of tendo-Achillis, 236.

of tendons of forearm, 222.

of the body, 216.

of upper extremity, 218.

pathology of, 209-10.

treatment of, 211-12.

Sacro-coccygeal sprain, causes of, 118.
Sacro - coccygeal sprain, coccygodynia a symptom in, 119. differential diagnosis of, 118. symptoms of, 118. treatment and prognosis of, 118.

Sacro-iliac ligaments, 118.

Sacro-lumbalis muscle, 35, 195, 206. dislocation and rupture of, 218.

symptoms and treatment of 218.

Sankey, Mr. R. H., 233.

Scaphoid bone, cases illustrating fracture of, 96-102.

clinical symptoms of, 98.

Stanford, Mr. Elliot, case of, 98101.

prognosis of, 102.

treatment of, 99-102.

Schlatter's disease, 134-40.

anatomy of, 134-5.

bursitis in, 138 .

detachment of epiphysis for tubercle of patella in Schlatter's disease, 138-9.

differential diagnosis of, 140 .

myositis ossificans in, 138-40.

prognosis of, 142 .

radiograms of, 137-9.

Swedish massage for, 138.

symptoms of, 135, 136, 138.

treatment of, 141-2.

X-rays in diagnosis of, 140 .

Sciatic nerve, injury to, 13.

Scott's dressing, 45, 253.

Seabury's plaster, 18, 225, 227, 255.

Seismo-therapy, 262.

Semilunar cartilages of knee, apparatus for preventing displacement of, 254.

author's method of operation for displacement of, 177 .

calcareous deposition in detached, 76.

changes observable in cartilages that have become detached or torn, 158.

drainage for, 181-2.

internal more liable to injury than external, 153.

factors concerned in, 153-4.

longitudinal splitting of internal, 151.

See Displaced semilunar cartilages. 
Semi-membranosus muscle, rupture of, 2229.

Semi-tendinosus muscle, rupture of, 229.

Separation of body of an internal semilunar cartilage from deep fibres of internal lateral ligament of joint, 133.

symptoms and results of, 133 .

Separation of epiphysis for anterior inferior spinous process of ilium, 2:22.2.

Sequela of sprains, $41-77$.

preventable, 41-60.

unavoidable, 60-77.

Serous effusion in joints, 10.

Simple sprain, 9-10,

passive movements in, $2 \overline{7}, 244$.

treatment of, compared with that of fracture-sprain, 39.

Simple strains, 18.

Shenton, Mr. E. W. H., 93.

Shoulder-joint, integrity of, dependent upon strength of its ligaments, \&c., $4,80$.

sprains of, 80 .

aspiration in treatment of, 83 .

causes of pain in. 81,83 .

complications of, 83.

diagnosis of, 82.

liability to atrophy of muscles in, $81,84$.

movements in, importance of early, 83-5.

permanent ill-effects of, 84 . surgical anatomy of, 80 . swelling of ten considerable in, 81 . symptoms of, 81 .

treatment of, 82,83 .

weakness of, 4 .

'Slipped cartilage,' 147.

subluxation of, 147 .

Soleus muscle, ossifying myositis in, 66-7.

Special sprains-

ankle-joint, 112 .

hip-joint, 109.

knee-joint, 111, 124.

shoulder-joint, 80

thumb, 104.

wrist, 91.

Splenius capitus muscle, 204.

Splints, prolonged and constant use of harmful, 42.
Splints, uses of, strictly limited, 42.

Sprain-dislocation, 12.

'Sprained elbow,' 85, 90. causation of, 89 .

exercises productive of, 90 .

symptoms of, 90.

Sprain-fracture, 1, 10, 16.

ankylosis as sequela of, 41, 60 .

causes of stiffness in, 40 .

passive movements in, 38, 244.

pathological significance of, 8 .

Sprain, in region of knee-joint, 109. of elbow-joint, 85, 90.

of external lateral ligament of knee, $15,132$.

of the body, 117 .

sacro-coccygeal, 118.

with immediate swelling, 9, 19.

Sprain with fracture, 10, 244.

Sprains, anæsthetics in treatment of, 14.

anatomical conditions underlying, 1 .

articular, 9 .

classical conception of, 8 .

cold in treatment of, 19.

complicated with hæmatomata, 14.

complicated with injuries to bone, 10.

complicated with injury to nerve, $12,35$.

numbness a symptom of, 12, 35 . crepitus as a symptom of, 15 .

definition of, 7 .

diagnosis of, 14.

divergent views on treatment of, 1 . elastic pressure in treatment of, 20-4.

evaporating lotions in treatment of, 51 .

general considerations of, 6 .

hæmatomata in, 10.

massage and movements in treatment of, 240-51.

of acromio-clavicular joint, 79.

of hip, 109.

of internal lateral ligament of knee, 130-2.

of knee, 120-7.

of muscle, 9.

of shoulder-joint, 80 .

of tarso-metatarsal joint of great toe, 116. 
Sprains, of thumb, 104.

paresis and paralysis as sequelæ of, 61 .

pathological conditions of, 1 .

rest in treatment of, 41.

'Rider's sprain,' 43.

treatment of deferred swelling in, 27.

treatment of improper union of muscles and tendons as sequela of, 59 .

unavoidable sequelæ of, 60-77.

warm applications in treatment of, $19,82$.

with fracture, treatment of, 38.

Spring-truss for knee, 254.

'Sprinter's thigh,' 211.

Stanford, Mr. Elliot, fracture of scaphoid bone by, 98 .

description of, 98-101.

Static electricity in treatment of nerve lesions, 37 .

Sterno-clavicular joint, sprain of, 78. anatomy of injury, 78 .

symptoms and treatment of, 78 .

Stiffness in joints, 41.

causes of, 42 .

range of movement in stiff-joints, 247.

Strain, anatomical conditions underlying, 1.

of internal lateral ligament of knee, 130.

the simplest form of sprain, 9 .

treatment of simple, 18.

Strapping, for knee, ankle, foot, and shoulder, 258-9.

for muscles of chest, rectus abdominis, muscles of thigh, muscles of calf, 255-8.

for strains, 18.

of injured muscles, advantages of, 225-8.

Strata, fascial and inter-muscular, 3.

Strong, Dr. R. H., 263.

Styloid processes of radius, fracture of, $92-4$.

Subdeltoid bursa muscle, 81 .

hæmorrhage into, in sprains of shoulder-joint, 81 .

Subluxation, 147. of head of radius, 88.

Surgical anatomy, general, of joints, 2-5.
Surgical anatomy, of inter-articular fibro-cartilage of knee, 122.

of knee-joint, 120.

Swedish massage in Schlatter's disease, 138.

Synovia, function of, 2.

Synovial cavity, stomata in connexion with, 3.

Synovial fringes, 47, 73, 192.

Synovial membrane, appearance of, in relapsing synovitis, 48 .

folding of, 2.

villi or papillæ 0 ? 2,3 .

Synovitis, acute traumatic of knee, 127.

chronic, hypertrophy of papillæ in, 75 .

exploratory arthrotomy in treatment of, 47.

drainage for, 48 .

forcible movements in treatment of, 46.

formation of loose bodies in, 75 .

general relaxation of ligaments as result of, 46 .

means of preventing, 46 .

presence of adhesions as a cause of, 46.

recurring and relapsing, 55 .

treatment of, 46.

use of anæsthetics in, 46.

Tarsal joint, 4.

Tarso-metatarsal joint of great toe, 116.

Temperature, high degrees of, application in treatment of sprains, 259.

uses of, 259.

Temperature charts, in operation for displaced semilunar cartilage, 184-7.

for exploratory arthrotomy, 49 .

Temporo-maxillary joint, 12 .

inter-articular cartilage of, 12.

Tendo-Achillis, ossification in, 238.

rupture of, 236 .

symptoms, diagnosis, and treatment of, 236-9.

Tendons, displacement or dislocation of, 198.

examples of, 198.

long of biceps, 203.

small, at back of neck, 204-6. 
Tendons, displacement or dislocation of the peroneal, 199, 203.

ilio-psoas, rupture of, 218.

of forearm, rupture of, 222 .

popliteus, 124, 155.

quadriceps extensor, 121, 124.

rupture of, causes of, 209-11.

treatment of improper union of, as sequela of sprains, 59 .

'Tennis elbow,' 194.

Tension in joints, aspiration in treatment of, 32,33 .

local heat as symptom of, 32 .

local treatment of, 31-2.

uses of elastic pressure in, 33 .

Tensor vagina femoris muscle, 196.

Thomson, Professor Alexis, 142.

Thumb, carpo-metacarpal joints of, injuries to, 104.

metacarpo-phalangeal joints of, injuries of, 104.

differential diagnosis of, 104-5. symptoms of, 104.

sprain-fracture of, 106.

treatment of, 106.

sprains of, 104.

causes of, 104.

treatment of simple sprain of, 106.

prognosis of, 106.

Tibialis anticus muscle, 196.

Tibialis posticus muscle, 198.

Tincture of iodine, 253.

Transverse processes of dorsal and lumbar vertebræ, fracture of, 207.

dangers of, 209.

prognosis of, 209.

symptoms of fracture, 207-8.

treatment of, 209.

X-rays in diagnosis of, 209.

Traumatic hydrops, etiology of, 127.

Treatment of recurring and relaps. ing displacement of semilunar cartilages, 173-4.

Treatment of sprains, 18.

Triceps muscles, rupture of, 218 .
Tuberculosis as sequela and complication of sprain, 30 .

Tumour as sequela and complication of sprain, 16.

Tunnicliffe, Dr., 241.

Unavoidable sequelæ of sprains, 60-77.

classification of, 61 .

Unregistered practitioners, 7.

Upper extremity, rupture of muscles of, 218.

causes, symptoms, and treatment of, 218 .

Vasogen-iodine, 30, 252.

Villi of synovial membranes, development of cartilage or bone from, 3.

diseased conditions of, 2.

hypertrophy in chronic synovitis, 75.

Warm applications in treatment of sprains, 19, 82 .

'Weak joint,' 41, 50.

Whitelocke, Mr. R. H. Anglin, $162,223$.

Wicket-keepers, injuries to fingers of, 58.

'Wobbly joint,' 41, 50.

local treatment of, 52 .

Workmen's Compensation Act, 6, 17.

Wrist, 4.

leather straps for, 254 .

Wrist-joint, sprain of, in 'pulled elbow', 89.

simple sprain of, 91 .

differential diagnosis of, 92 .

etiology of, 92 .

symptoms of, 92 .

treatment of, 96 .

swelling in sprain of, 96 .

$\mathrm{X}$-rays as an aid to diagnosis, 8, 16 , $40,58,64,67,87,94,96,98$, $106,107,112,232$. 


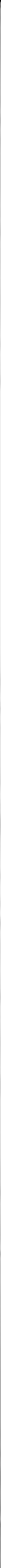





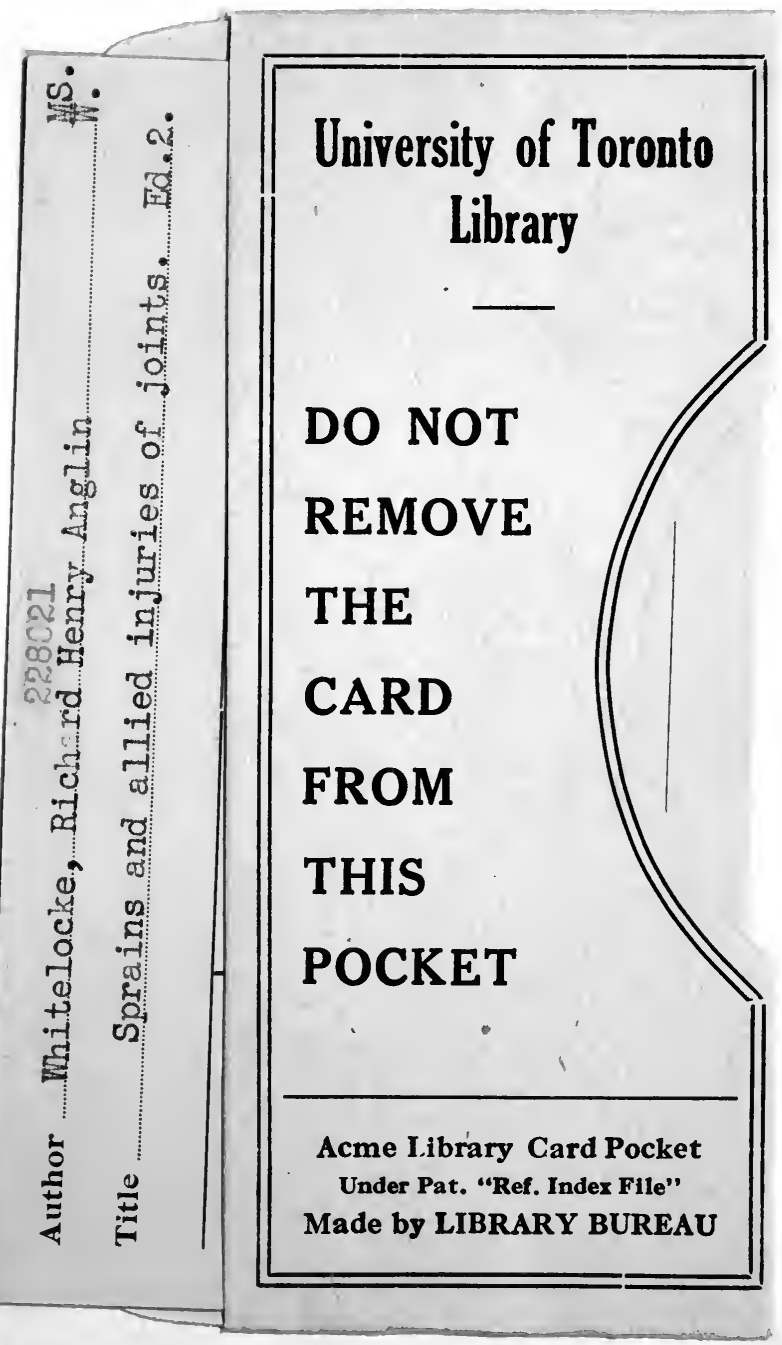




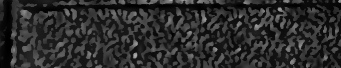

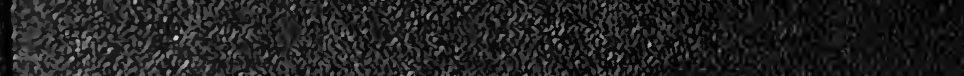
Hor.

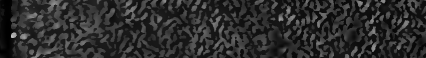

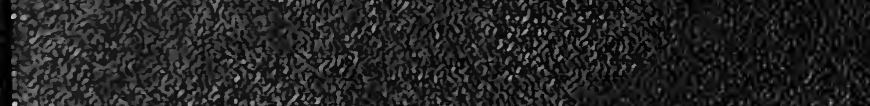

-

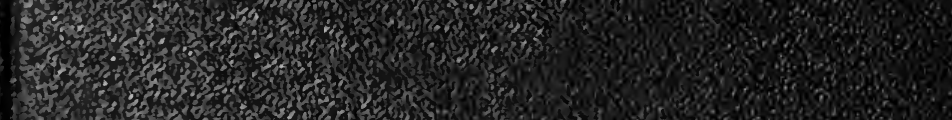

27.

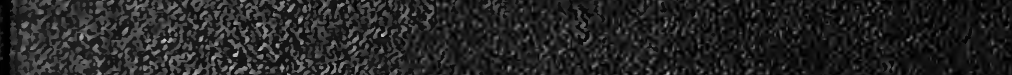

T.

73 .

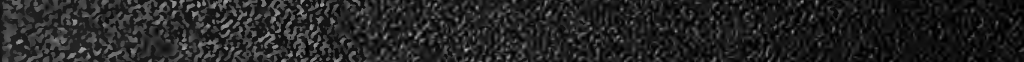

- 0 .

(5)

-3.

5 (1) -

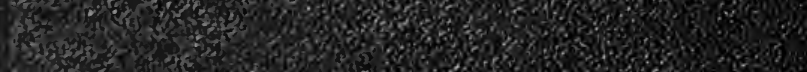

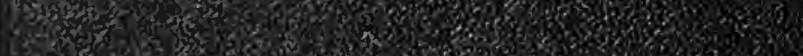

(3)

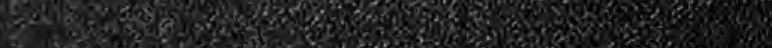

40 ar

6-1.2.

3.

-

2.

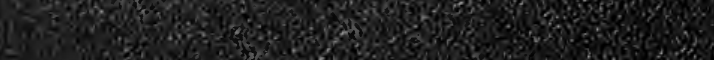

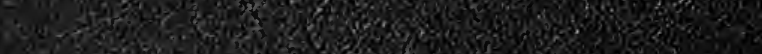

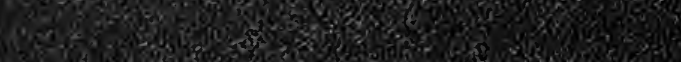

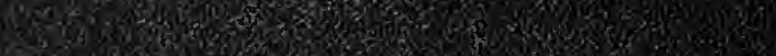

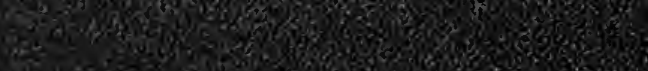

*

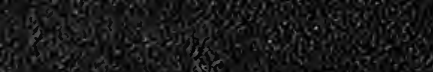

a 2 s 